\title{
MEASUREMENT OF THE BEAM ASYMMETRY FOR THE ETA AND ETA PRIME MESONS WITH THE GLUEX EXPERIMENT
}

\author{
A Thesis \\ Submitted to the Faculty of Graduate Studies and Research \\ In Partial Fulfillment of the Requirements \\ FOR THE DEGREE OF \\ Doctor of Philosophy \\ IN \\ PHYSICS \\ UNIVERSITY OF REGINA
}

by

Tegan Donald Beattie

Regina, Saskatchewan

March, 2019

(c) Copyright 2019: T. D. Beattie 


\section{Abstract}

The GlueX Experiment in Hall D at Jefferson Lab ultimately aims to provide evidence of hybrid mesons, $q \bar{q}$ pairs with gluonic excitations, which are predicted by quantum chromodynamics calculations on the lattice. GlueX features a linearlypolarized photon beam incident on a liquid hydrogen target and a nearly- $4 \pi$ hermetic

detector capable of measuring positions and momenta of both charged and neutral final state particles generated in the target. The short-term physics goals of GlueX are to measure observables and properties of the known particles, especially those that are likely decay products of hybrids, such as the $\eta$ and $\eta^{\prime}$ mesons. An overview of the detector subsystems and the long-term physics goals of GlueX are presented, along with analyses on the $\Sigma$ beam asymmetry of the $\eta$ and $\eta^{\prime}$ mesons.

The measurements of $\Sigma$ are presented versus the four-momentum transfer squared using a photon beam energy of $8.2-8.8 \mathrm{GeV}$ in the three main decay channels of the $\eta\left(\eta \rightarrow 2 \gamma, \eta \rightarrow \pi^{+} \pi^{-} \pi^{0}\right.$, and $\left.\eta \rightarrow 3 \pi^{0}\right)$ and the largest branching fraction decay channel of the $\eta^{\prime}\left(\eta^{\prime} \rightarrow \pi^{+} \pi^{-} \eta\right.$, with $\left.\eta \rightarrow 2 \gamma\right)$. These are the first results for the beam asymmetry of the $\eta^{\prime}$ measured at this beam energy. The results, which are in agreement with past published measurements for the $\eta$ by GlueX and with modern theory predictions for the $\eta$ and $\eta^{\prime}$, indicate a dominance of natural parity exchange in the resonance production mechanism. 


\section{Acknowledgements}

I would like to start by thanking my supervisor, Dr. Zisis Papandreou, for his help with everything: suggesting analysis ideas, providing financial support, explaining physics concepts, editing this thesis along with many other documents, and organizing useful discussions with detector and analysis experts.

Thanks to Dr. Justin Stevens, for helping me understand the GlueX analysis code by providing examples and answering many questions, and for guiding the beam asymmetry analyses. Thanks also to Dr. Dave Mack, for lending guidance in error analysis and systematic checks.

I owe many thanks to Dr. Andrei Semenov, for analysis insights, technical assistance with ROOT, and diagnosing issues with my computer whenever I broke it.

To the rest of my advisory committee, Dr. George Lolos, Dr. Garth Huber, Dr. Nader Mobed, and Dr. Shaun Fallat: thank you for reviewing my work, pinpointing errors, suggesting additions, and helping to clarify my understanding of certain topics.

Thanks to Dr. Elton Smith and the rest of the Hall D staff at JLab, for many discussions regarding analysis and software, and thanks to Mr. William McGinley, for tracking down systematic differences between our analyses and providing extremely helpful cross-checks on data sets and analysis code.

Finally, I am deeply thankful to my family. The support they have provided has afforded me the luxury to focus exclusively on school work and research during my time as a graduate student. 


\section{Contents}

Abstract $\quad$ i

Acknowledgements $\quad$ ii

Table of Contents $\quad$ iii

List of Figures $\quad$ vi

List of Tables $\quad$ XV

List of Abbreviations $\quad$ xvii

1 Introduction $\quad 1$

1.1 The Standard Model . . . . . . . . . . . . . . . . . . . . . 2

1.2 The Quark Model . . . . . . . . . . . . . . . . . . . . . 5

1.3 Exotic and Hybrid Mesons _. . . . . . . . . . . . . . . . . . . 9

1.3.1 Models and Predictions for Exotics and Hybrids . . . . . . . . . . . 12

1.3.1.1 Constituent Gluon Model . . . . . . . . . . . . . . . . . . . . 13

1.3.1.2 Lattice QCD . . . . . . . . . . . . . . . . . . 14

1.3.1.3 Flux Tube Model . . . . . . . . . . . . . . . . . 15

1.3.1.4 Exotic Hybrid Meson Predictions . . . . . . . . . . . . . 16

1.4 Existing Evidence for Non-Quark Model Mesons . . . . . . . . . . . . 18

1.5 Exotic Production with the GlueX Experiment . . . . . . . . . . . . . 22

1.6 Current GlueX Efforts . . . . . . . . . . . . . . . . . . . . . . . . 23

1.6.1 Pseudoscalar Beam Asymmetry . . . . . . . . . . . . . . 24

1.6.2 Predictions of $\eta$ and $\eta^{\prime}$ Beam Asymmetries . . . . . . . . . . . . . . 27

2 The GlueX Experiment 31

2.1 CEBAF at Jefferson Lab . . . . . . . . . . . . . . . . . . . . 31

2.2 Hall D . . . . . . . . . . . . . . . . . . . . . . . . 35

2.2.1 Beam Line. . . . . . . . . . . . . . . . . . . . . . . . . . . . . . . . . . . . . . 35

2.2.1.1 Polarized Photon Beam . . . . . . . . . . . . . . . 35

2.2.1.2 Tagger Hodoscope and Microscope . . . . . . . . . . . . . . . 39

2.2.1.3 Triplet Polarimeter . . . . . . . . . . . . . . . . . . 40

2.2.1.4 Pair Spectrometer . . . . . . . . . . . . . . . . . . . . . . . . . . . . . . 42

2.2.1.5 Liquid Hydrogen Target . . . . . . . . . . . . . . . . . . 43 
2.2.2 Charged Particle Tracking . . . . . . . . . . . . . . . . . 43

2.2.2.1 Central Drift Chamber . . . . . . . . . . . . . . . . 45

2.2.2.2 Forward Drift Chamber . . . . . . . . . . . . . . . . . 46

2.2 .3 Calorimetry . . . . . . . . . . . . . . . . . . . . . . 48

2.2.3.1 Barrel Calorimeter . . . . . . . . . . . . . . . . . . . 49

2.2.3.2 Forward Calorimeter . . . . . . . . . . . . . . 52

2.2 .4 Particle Identification . . . . . . . . . . . . . . . . . . 55

2.2.4.1 Start Counter . . . . . . . . . . . . . . . . . 55

2.2.4.2 Time-of-Flight . . . . . . . . . . . . . . . 56

2.2.4.3 Recent Upgrade: DIRC . . . . . . . . . . . . . . . . . 58

2.3 GlueX Summary . . . . . . . . . . . . . . . . . . . . . 61

3 Data Analysis $\quad 62$

3.1 Event Selection . . . . . . . . . . . . . . . . . . . . . . 64

3.1 .1 Phase I Selection . . . . . . . . . . . . . . . . . . . . . . 64

3.1 .2 Phase II Selection . . . . . . . . . . . . . . . . . . . . . . . 68

3.1 .3 Phase III Selection . . . . . . . . . . . . . . . . . . . . . 69

3.2 Beam Asymmetry Analysis Methodology . . . . . . . . . . . . . 76

3.2 .1 Accidental Subtraction . . . . . . . . . . . . . . . . . 78

3.2 .2 Side-Band Asymmetry Correction . . . . . . . . . . . . . . . . 79

3.3 Asymmetry Fit Parameters . . . . . . . . . . . . . . . . . . . 80

3.3 .1 Flux Ratio. . . . . . . . . . . . . . . . . . . . . . . 81

3.3 .2 Beam Polarization . . . . . . . . . . . . . . . . . . . . . . 81

3.3 .3 Phase Offset. . . . . . . . . . . . . . . . . . . . . . 83

3.4 Beam Asymmetry Analysis Workflow . . . . . . . . . . . . . . . 84

4 Beam Asymmetry Results $\quad 85$

$4.1 \eta$ Beam Asymmetry . . . . . . . . . . . . . . . . . . . . . . 85

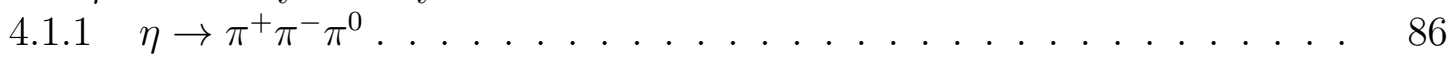

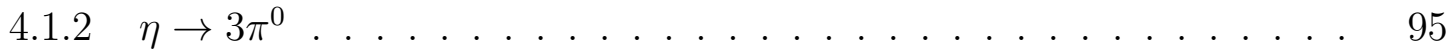

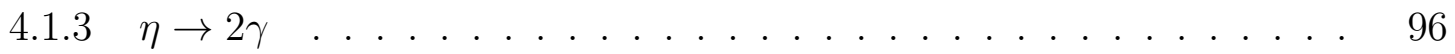

4.1.4 Past Results and Models . . . . . . . . . . . . . . . . . . . . 101

$4.2 \eta^{\prime}$ Beam Asymmetry . . . . . . . . . . . . . . . . . . . . . . . . . 104

$4.2 .1 \eta^{\prime} \rightarrow \pi^{+} \pi^{-} \eta, \eta \rightarrow 2 \gamma \ldots \ldots \ldots \ldots \ldots \ldots$

4.3 Beam Asymmetry Ratio . . . . . . . . . . . . . . . . . . . . 111

5 Systematic Studies $\quad 114$

5.1 Kinematic Fit Confidence Level Cut Study . . . . . . . . . . . . . 115

$5.2 \eta^{\prime}$ Asymmetry Systematic Studies . . . . . . . . . . . . . . . . . . . 121

$5.2 .1 \quad$ Side-Band Asymmetry . . . . . . . . . . . . . . . . . . . . 122

5.2.1.1 $\quad \eta$ Side-Band Asymmetry . . . . . . . . . . . . . . . . . . . . . 124

5.2 .2 Cut Variations . . . . . . . . . . . . . . . . . . . . . . . . . . . 125

5.2 .3 Instrumental Asymmetry . . . . . . . . . . . . . . . . . . . 128

5.2 .4 Phase Offset . . . . . . . . . . . . . . . . . . . . . . . . 130

5.2 .5 Flux Ratio. . . . . . . . . . . . . . . . . . . . . . . . . . 131 
5.2.6 Fit Function Variations . . . . . . . . . . . . . . . . . . . . . . 132

5.2.7 Finite $\phi$-Dependent Yield Bin Size . . . . . . . . . . . . . 135

$5.2 .8 \quad \eta^{\prime}$ Systematics Summary . . . . . . . . . . . . . . . . 135

6 Conclusions and Future Direction 137

$\begin{array}{lr}\text { References } & 139\end{array}$

$\begin{array}{ll}\text { A Nominal Event Selection Cuts } & 147\end{array}$

$\begin{array}{lr}\text { B Additional Analysis Plots } & 148\end{array}$

C Summary of Beam Asymmetry Results 155 


\section{List of Figures}

1.1 The fundamental particles of SM physics. Quarks and leptons are arranged by generation. Each particle's mass, spin, and electric charge are noted. Particles with colour charge are also indicated. . . . . . . .

1.2 Weight diagrams for the pseudoscalar (a) and vector (b) mesons in $\mathrm{SU}(4)$. The light meson multiplets exist on the central plane $(C=0)$ along with the $c \bar{c}$ states, the $\eta_{C}$ and $J / \Psi$. Images from Reference [6].

1.3 Lattice gauge theory simulation in $\mathrm{SU}(2)$. Shown is the action density created by two static colour sources (infinitely heavy quarks, for example) separated by $1.2 \mathrm{fm}$ on a $32^{4}$ point lattice (see Section 1.3.1.2). Plot from Reference [8]. . . . . . . . . . . . . . . . .

1.4 A sketch showing the field lines and force between two charge sources in electrodynamics (a) and QCD (b). . . . . . . . . . . .

1.5 A sketch depicting the production of a pair of quarks. Rather than liberating a quark from a hadron, the energy in the flux tube becomes large enough with increasing distance to generate a new $q \bar{q}$ pair, resulting in two distinct hadrons. . . . . . . . . . . . . .

1.6 The LQCD meson spectrum, displayed as state mass versus quantum numbers $\left(J^{P C}\right)$. The height of a state indicates the error on the mass. Octet/singlet mixing angle is indicated by black and green contributions to isoscalar mesons. Candidates for the lightest hybrid supermultiplet are highlighted in orange $[14]$. . . . . . . . . . . . . . . .

1.7 An example of the isobar model. Creation of a three-pion resonance (X, with quantum numbers $J^{P C}$ ) is modelled by creation of a pion and an isobar $(\mathrm{R})$ with the isobar subsequently decaying to two pions. The total cross section is the sum of all possible contributions. . . . . . .

1.8 GlueX $\Sigma$ beam asymmetry for photoproduction of the $\pi^{0}$ (a) and the $\eta$ (b) off the proton at $E_{\gamma}=9 \mathrm{GeV}$ (black filled circles) [63]. Uncorrelated systematic errors are indicated by grey boxes and combined statistical and systematic uncertainties are given by black error bars. SLAC results for the $\pi^{0}$ for an average beam energy of $10 \mathrm{GeV}$ are also shown [74] (blue open circles), along with various model predictions (Laget [75, 76], JPAC [77, 78], Donnachie [79], and Goldstein [80]). . 
1.9 Theory curves produced by JPAC for $\Sigma_{\eta^{\prime}} / \Sigma_{\eta}$. The two curves derive from two different model assumptions for the axial vector radiative decay strengths, with the similarly coloured bands representing the $\pm 1 \sigma$ error on each curve. More detailed information regarding the model can be found in the published JPAC paper [59]. . . . . . . . .

2.1 CEBAF at Jefferson Lab. A high-frequency electron beam is accelerated to high energy and supplied to up to four experimental halls simultaneously. . . . . . . . . . . . . . . . .

2.2 Thickness scan (a) and rocking curve (b) from GlueX diamond JD70100, the radiator used during data taking in the spring of 2017. Images from Reference [88]. . . . . . . . . . . . . . . . . .

2.3 Bragg reflection of a plane wave on a crystal lattice. An incident plane wave generates a coherent reflected plane wave when Bragg's Law (described in the Figure) is satisfied. . . . . . . . . . . . . . . .

2.4 (Left) Theoretically calculated beam rate of the GlueX photon beam as a function of energy. (Right) Polarization of that photon beam as a function of energy. Simulated using a radiator thickness of $10^{-4}$ radiation lengths and a $3 \mu \mathrm{A}$ beam current. In both figures, the dashed line is the result with no beam collimation while the solid line includes a collimator $3.4 \mathrm{~mm}$ in diameter placed $80 \mathrm{~m}$ from the radiator. Figure from Reference [90]. . . . . . . . . . . . . . . . . . . . . .

2.5 Rendering of the tagger dipoles and the focal plane of the TAGH and TAGM. Bent lines incident on the focal plan show electron trajectories for electrons of varying energies. Figure from Reference [89]. . . . . .

2.6 Polarization of the photon beam as measured by the TPOL as a function of energy for the four diamond orientation data sets. Statistical errors on each point are represented by vertical error bars. . . . . . .

2.7 Schematic of the Pair Spectrometer. The electron-positron pair is separated by a dipole and the momenta of both particles is measured with a pair of hodoscopes. Figure from Reference [92]. . . . . . . . . . . .

2.8 Photon beam energy distribution for PS events in coincidence with a TAGH or TAGM hit for the four photon polarization angles used. . .

2.9 Schematic of the liquid hydrogen target used for the GlueX Experiment. The liquid hydrogen is contained in the orange-coloured cone. Figure from Reference [93]. . . . . . . . . . . . . . . . . . .

2.10 Overview of detector subsystems in the GlueX detector. Figure from

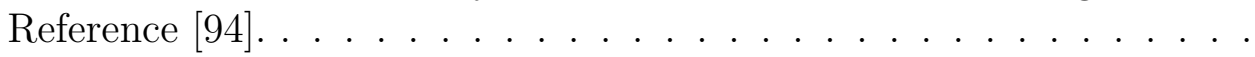

2.11 View from upstream of the CDC straw configuration. Depicted are the radial layers of axial straws (black dots) and stereo straws (red and blue dots, $\pm 6^{\circ}$ respectively). Figure from Reference [95]. . . . . 
2.12 CDC $\mathrm{dE} / \mathrm{dx}$ versus charged track momentum. The horizontal band below momenta of $1 \mathrm{GeV}$ is a result of lighter charged particles like electrons, pions, and kaons. The curved band is a result of protons. Above $1 \mathrm{GeV}$ or so, the two bands become indistinguishable. The red curve shows an extremely loose cut meant to exclude obvious light particles while preserving all proton candidates at low momentum in the early stages of an analysis. . . . . . . . . . . . . . .

2.13 (a) Front view of an FDC package. Wires are shown as vertical lines. Two cathode strip planes lie at $\pm 75^{\circ}$ with respect to the wires, one on either side of the wires ( $U$ and $V$ strips). (b) Side view of an FDC package. Wire planes are shown as dashed lines, while cathode planes are shown as solid lines. A package contains six sets of cathode strip detectors, each rotated $60^{\circ}$ from the previous one. Figure from

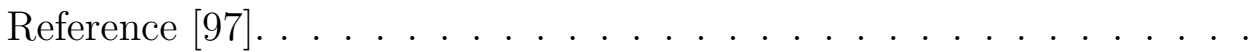

2.14 (a) Macro dimensions of the BCAL. (b) cross sectional (top-half only) view of the BCAL showing it's position with respect to the hydrogen target. (c) End view of the BCAL showing the 48 trapezoidal modules together forming the cylinder. (d) End view of one BCAL module showing the 40 light guide footprints and the layer-wise summing scheme used to produce the final 16 readout channels. Figure from Reference [98]. . . . . . . . . . . . . . . . . . .

2.15 Measured speed ( $\beta$, the particle speed divided by the speed of light) of positively charged particles found using BCAL shower time information versus particle momentum. The three curves, from top to bottom, correspond to the expected distributions of pions, kaons, and protons.

2.16 Rendering of the GlueX FCAL, showing the partially-stacked lead-glass blocks, the beam hole, and the acrylic planes used for gain monitoring. Figure from Reference [100]. . . . . . . . . . . . . . . . .

2.17 Ratio of energy, as measured by the FCAL, to charged particle track momentum versus track polar angle. Using energy information from the FCAL, identification of hits as being from electrons or positrons as opposed to hadrons is possible. Figure from Reference [102]. . . . . . 54

2.18 Schematic view of the Start Counter. Figure from Reference [103]. . .

2.19 Early conceptual rendering of the TOF wall. Note that the half-width scintillator bars on either side of the beam hole are not represented in this rendering. Figure from Reference $[105]$. . . . . . . . . . . . .

2.20 Speed, as measured by the TOF, of positively charged particles versus momentum showing separation of the positron, pion, kaon, and proton bands in various momentum ranges. The faint, horizontal band around $\beta=0.8$ is caused by 'accidentally-tagged' (see Figure 3.1 and associated text) positron and pion tracks. Figure from Reference [105]. 
2.21 (Left) Schematic of the expansion volume and optical box mirrors. Cherenkov light from the BaBar boxes enter from the bottom left and is directed by the mirrors to the photosensor array at the top right. (Right) Three-dimensional rendering of the optical box coupled to two bar boxes. Figure from Reference [107]. . . . . . . . . . . . . . . . . .

2.22 Simulated PMT photosensor patterns for normally incident charged particles and an optical box mirror setup similar to that in Figure 2.21. (Left) Optical box of arbitrary length: the entire pattern is available without reflection. (Right) $1.4 \mathrm{~m}$ long optical box, as designed for GlueX, provides the same pattern with some folding due to reflection. Figure from Reference [107]. . . . . . . . . . . . . . . . . .

3.1 RF $\Delta$ t distribution. The central peak between the green delimiting lines contains the real photoproduction events and some contribution from accidentals. The smaller peaks are from accidental events. The six small peaks between the two sets of red delimiting lines are the peaks used for accidental subtraction, as described in Section 3.2.1. .

3.2 The z-position of the kinematically-fit vertex (left) and the x-position versus y-position of the kinematically-fit vertex (right). The red delimiting lines indicate the cut limits. . . . . . . . . . . . . .

3.3 A side-view of the GlueX detector showing only the BCAL and FCAL. Fiducial cuts (shown as red regions) are placed around the beam hole in the FCAL and around the FCAL-BCAL gap where reconstruction or calibration can be difficult or unreliable. . . . . . . . . . . . . . .

3.4 KinFit $\pi^{+} \pi^{-} \pi^{0}$ mass vs. measured $\pi^{0} \rightarrow 2 \gamma$ mass (left) and KinFit $\pi^{+} \pi^{-} \eta$ mass vs. measured $\eta \rightarrow 2 \gamma$ mass (right). The red delimiting lines indicate the cut limits on the $2 \gamma$ masses. . . . . . . . . . . . . .

3.5 Invariant mass spectra of the $\eta$ for the 3piq (top left), 3 pi0 (top right), and $2 \mathrm{~g}$ (bottom left) decay modes, and of the $\eta^{\prime}$ (bottom right). The effects on the mass spectra of various cuts are shown. . . . . . . . . .

3.6 The Gaussian distribution is a symmetric function around some mean, $\mu$, with variance $\sigma^{2}$ and amplitude $\mathrm{A}$. The integral under the curve in the range $\mu \pm \sigma, \mu \pm 2 \sigma$, and $\mu \pm 3 \sigma$ are displayed, along with the functional form. . . . . . . . . . . . . . . . .

3.7 Fits to the invariant mass spectra of the $\eta$ for the 3piq (top left), 3pi0 (top right), and $2 \mathrm{~g}$ (bottom left) decay modes, and of the $\eta^{\prime}$ (bottom right), to Equation 3.4. The exponential component of the fit function is replaced by a small constant term for the $3 \mathrm{pi0}$ decay mode (omitted from the fit statistics). The peak region used to select signal events for further analysis is displayed in each plot. These spectra are integrated over the entire range of $t$ studied, referred to as ' $t$-averaged.' . . . . .

3.8 Fits to the high-statistics $t$-averaged $\eta \rightarrow 2 \gamma$ SB yield asymmetry for 0/90 (left) and 45/135 (right) data sets allowing the phase offset, $\phi_{0}$, to float. The extracted phase offsets are then fixed in fits to all other yield asymmetries. 
4.1 $\pi^{+} \pi^{-} \pi^{0}$ mass spectra and dilution factors for four ranges of $t$ (all polarization orientations). The blue delimiting lines denote the peak region, and the purple delimiting lines denote the side-band region. . . . . .

$4.2 \eta \rightarrow \pi^{+} \pi^{-} \pi^{0} 0 / 90$ yield asymmetry distributions in the $\eta$ peak region for four ranges of $t \ldots \ldots \ldots \ldots . \ldots . . . \ldots$

$4.3 \eta \rightarrow \pi^{+} \pi^{-} \pi^{0} 0 / 90$ yield asymmetry distributions in the side-band region for four ranges of $t$. . . . . . . . . . . . . . . .

$4.4 \eta \rightarrow \pi^{+} \pi^{-} \pi^{0} 45 / 135$ yield asymmetry distributions in the $\eta$ peak region for four ranges of $t \ldots \ldots \ldots \ldots$. . . . . . . . . .

$4.5 \eta \rightarrow \pi^{+} \pi^{-} \pi^{0} 45 / 135$ yield asymmetry distributions in the side-band region for four ranges of $t \ldots \ldots \ldots \ldots$

4.6 $\eta$ beam asymmetries versus $t$ for the $\eta \rightarrow \pi^{+} \pi^{-} \pi^{0}$ decay channel, from left to right: $\Sigma_{\text {peak }}, \Sigma_{\mathrm{SB}}$, and $\Sigma_{\mathrm{COR}}$. 0/90 data are in blue (circles), 45/135 data are in red (squares). Errors are statistical only. . . . . . 90

$4.7 \quad \chi^{2} \mathrm{PDF}$ for various degrees of freedom, $n d f$. . . . . . . . . . . . . . 92

4.8 Weighted average of the $0 / 90$ and 45/135 side-band corrected beam asymmetries for the $\eta \rightarrow \pi^{+} \pi^{-} \pi^{0}$ decay channel. Vertical error bars are statistical only, and the height of the marked box represents the $2.1 \%$ normalization uncertainty. . . . . . . . . . . . .

$4.9 \eta \rightarrow 3 \pi^{0} 0 / 90$ yield asymmetry distributions in the $\eta$ peak region for four ranges of $t$. . . . . . . . . . . . . . . . . .

$4.10 \eta \rightarrow 3 \pi^{0} 45 / 135$ yield asymmetry distributions in the $\eta$ peak region for four ranges of $t \ldots \ldots \ldots \ldots$

$4.11 \eta$ beam asymmetries $\left(\Sigma_{\text {peak }}\right)$ versus $t$ for the $\eta \rightarrow 3 \pi^{0}$ decay channel. 0/90 data are in blue (circles), 45/135 data are in red (squares). Errors are statistical only.

4.12 Weighted average of the $0 / 90$ and 45/135 beam asymmetries for the $\eta \rightarrow 3 \pi^{0}$ decay channel. Vertical error bars are statistical only, and the height of the marked box represents the $2.1 \%$ normalization uncertainty. 96

$4.132 \gamma$ mass spectra, from the decay of the $\eta$, and dilution factors for four ranges of $t$ (all polarization orientations). The blue delimiting lines denote the peak region, and the purple delimiting lines denote the side-band region.

$4.14 \eta \rightarrow 2 \gamma 0 / 90$ yield asymmetry distributions in the $\eta$ peak region for

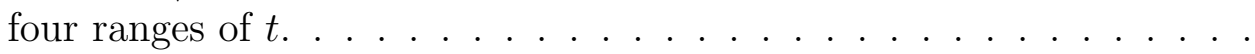

$4.15 \eta \rightarrow 2 \gamma 0 / 90$ yield asymmetry distributions in the side-band region for four ranges of $t \ldots \ldots \ldots \ldots \ldots$

$4.16 \eta \rightarrow 2 \gamma 45 / 135$ yield asymmetry distributions in the $\eta$ peak region for

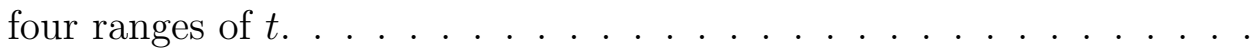

$4.17 \eta \rightarrow 2 \gamma 45 / 135$ yield asymmetry distributions in the side-band region

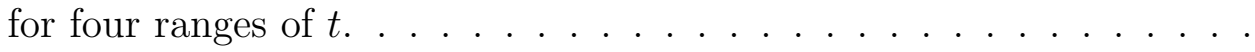

$4.18 \eta$ beam asymmetries versus $t$ for the $\eta \rightarrow 2 \gamma$ decay channel, from left to right: $\Sigma_{\text {peak }}, \Sigma_{\mathrm{SB}}$, and $\Sigma_{\mathrm{COR}}$. 0/90 data are in blue (circles), 45/135 data are in red (squares). Errors are statistical only. . . . . . . . . . . 100 
4.19 Weighted average of 0/90 and 45/135 side-band corrected beam asymmetries for the $\eta \rightarrow 2 \gamma$ decay channel. Vertical error bars are statistical only, and the height of the marked box represents the $2.1 \%$ normalization uncertainty. . . . . . . . . . . . . . . .

$4.20 \eta$ beam asymmetries versus $t$ for all three $\eta$ decay channels studied in this thesis (with vertical error bars representing statistical errors and the height of the marked box representing the $2.1 \%$ normalization uncertainty) overlayed with past (published 2017) GlueX results (with vertical error bars representing total errors and grey boxes representing contributions from systematic errors) and the theoretical models from Figure 1.8. . . . . . . . . . . . . . . . . . . . . .

$4.21 \eta$ beam asymmetries versus $t$ for the weighted average of the three $\eta$ decay channels studied in this thesis (with vertical error bars representing statistical errors and the height of the marked box representing the $2.1 \%$ normalization uncertainty) overlayed with past (published 2017) GlueX results (with vertical error bars representing total errors and grey boxes representing contributions from systematic errors) and the theoretical models from Figure 1.8. . . . . . . . . . . . . . . . . . 103

$4.22 \pi^{0} \pi^{0} \eta$ mass spectrum (all $t$, all polarization orientations). . . . . . . . 104

$4.232 \gamma$ mass spectrum, from the decay of the $\eta^{\prime}$, (all $t$, all polarization orientations). . . . . . . . . . . . . . .

$4.24 \pi^{+} \pi^{-} \eta$ mass spectra and dilution factors for four ranges of $t$ (all polarization orientations). The blue delimiting lines denote the peak region, and the purple delimiting lines denote the side-band region. . . . . . 106

$4.25 \eta^{\prime} \rightarrow \pi^{+} \pi^{-} \eta 0 / 90$ yield asymmetry distributions in the $\eta^{\prime}$ peak region

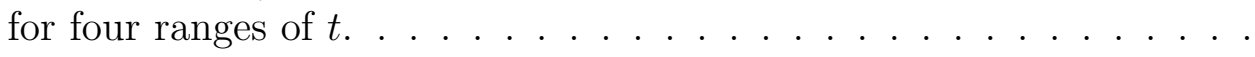

$4.26 \eta^{\prime} \rightarrow \pi^{+} \pi^{-} \eta 0 / 90$ yield asymmetry distributions in the side-band region for four ranges of $t$. . . . . . . . . . . . . . . . . 108

$4.27 \eta^{\prime} \rightarrow \pi^{+} \pi^{-} \eta 45 / 135$ yield asymmetry distributions in the $\eta^{\prime}$ peak region for four ranges of $t \ldots \ldots \ldots \ldots$

$4.28 \eta^{\prime} \rightarrow \pi^{+} \pi^{-} \eta 45 / 135$ yield asymmetry distributions in the side-band region for four ranges of $t \ldots \ldots \ldots \ldots$

$4.29 \eta^{\prime}$ beam asymmetries versus $t$ for the $\eta^{\prime} \rightarrow \pi^{+} \pi^{-} \eta$ decay, from left to right: $\Sigma_{\text {peak }}, \Sigma_{\mathrm{SB}}$, and $\Sigma_{\mathrm{COR}}$. 0/90 data are in blue (circles), 45/135 data are in red (squares). Errors are statistical only. . . . . . . . . . 109

4.30 Weighted average of 0/90 and 45/135 side-band corrected beam asymmetries for the $\eta^{\prime} \rightarrow \pi^{+} \pi^{-} \eta$ decay channel. Vertical error bars are statistical only, and the height of the marked box represents the $2.1 \%$ normalization uncertainty. The purple boxes on each point indicate the systematic error assigned to that point. . . . . . . . . . .

$4.31 \Sigma_{\eta^{\prime}} / \Sigma_{\eta}$ versus $t$, found as the ratios of data from the $\eta^{\prime}$ decay channel and the three $\eta$ decay channels analyzed. The two theoretical curves from JPAC (from Figure 1.9) are overlayed for direct comparison of data to theory. Errors are statistical only. 
$4.32 \Sigma_{\eta^{\prime}} / \Sigma_{\eta}$ versus $t$, found as the ratios of data from the $\eta^{\prime}$ decay channel and the weighted average of the three $\eta$ decay channels analyzed. The two theoretical curves from JPAC (from Figure 1.9) are overlayed for direct comparison of data to theory. Errors are statistical only. . . . .

$5.1 \eta \rightarrow \pi^{+} \pi^{-} \pi^{0}$ dilution factor, $f$, for the studied bins of $t$ versus kinematic fit confidence level cut. . . . . . . . . . . . . . . . .

$5.2 \eta \rightarrow \pi^{+} \pi^{-} \pi^{0}$ average SB-corrected $\Sigma$ asymmetries for the studied bins of $t$ versus kinematic fit confidence level cut (left) and statistical errors on those asymmetries (right). . . . . . . . . . . . . .

$5.3 \eta \rightarrow 3 \pi^{0}$ average SB-corrected $\Sigma$ asymmetries for the studied bins of $t$ versus kinematic fit confidence level cut (left) and statistical errors on those asymmetries (right). . . . . . . . . . . . . .

$5.4 \eta \rightarrow 2 \gamma$ dilution factor, $f$, for the studied bins of $t$ versus kinematic fit confidence level cut. . . . . . . . . . . . . . . . . . . . . 117

$5.5 \eta \rightarrow 2 \gamma$ average SB-corrected $\Sigma$ asymmetries for the studied bins of $t$ versus kinematic fit confidence level cut (left) and statistical errors on those asymmetries (right). . . . . . . . . . . . . . .

$5.6 \quad \eta^{\prime} \rightarrow \pi^{+} \pi^{-} \eta$ dilution factor, $f$, for the studied bins of $t$ versus kinematic fit confidence level cut. . . . . . . . . . . . . . . . .

$5.7 \eta^{\prime} \rightarrow \pi^{+} \pi^{-} \eta$ average SB-corrected $\Sigma$ asymmetries for the studied bins of $t$ versus kinematic fit confidence level cut (top left), the statistical errors on those asymmetries (top right), the systematic uncertainties on those asymmetries to do with the choice of $\Sigma_{\mathrm{SB}}$ (bottom left), and the total error (quadratic sum of statistical and systematic, bottom right). . . . . . . . . . . . . . . . . . .

5.8 Resultant $45 / 135 \Sigma_{S B}$ for various mass bins with a constant (red), linear (magenta), and exponential (cyan) fit. A range of values from extrapolations of the fits down to $0.958 \mathrm{GeV}$ is displayed. . . . . . . .

5.9 Resultant $0 / 90 \eta \rightarrow \pi^{+} \pi^{-} \pi^{0} \Sigma_{S B}$ for various mass bins in three ranges of $t$. . . . . . . . . . . . . . . . . . . . . . . . . . . . . . . . . . . . . . 124

5.10 Resultant $0 / 90 \eta \rightarrow 2 \gamma \Sigma_{S B}$ for various mass bins in three ranges of $t .125$

5.11 Average $\eta^{\prime}$ SB-corrected asymmetries for nominal (black open circles with error bars) and varied (coloured filled circles) cuts. Colours are as in the legend shown in Figure 5.12. . . . . . . . . . . . .

5.12 Legend for cut systematics plots (Figures 5.11, B.9, and B.10) and instrumental asymmetry plots (Figures 5.13, B.11, B.12, and B.13). .

$5.130 / 90 \eta^{\prime}$ peak region asymmetries for nominal (black open circles with error bars) and varied (coloured filled circles) cuts. Colours are as in the legend shown in Figure 5.12. . . . . . . . . . . . . . .

5.14 Average SB-corrected $\eta^{\prime}$ asymmetries for nominal fit parameters (black open circles with error bars) and floating $\phi_{0}$ fit (red filled circles). . . 
5.15 Histogram of differences between floating $\phi_{0}$ fit asymmetries and nominal fit asymmetries presented in Figure 5.14. The width of this distribution guides a selection of systematic uncertainty applied to the

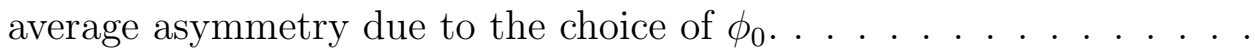

5.16 Average SB-corrected $\eta^{\prime}$ asymmetries for nominal fit parameters (black open circles with error bars), a 5\% increase in both $0 / 90$ and 45/135 flux ratios (red filled circles), and a $5 \%$ decrease in those flux ratios (blue squares). . . . . . . . . . . . . . . . .

5.17 Histogram of differences between varied $F_{R}$ fit asymmetries and nominal fit asymmetries presented in Figure 5.16. The width of this distribution guides a selection of systematic uncertainty applied to the average asymmetry due to the PS determination of $F_{R}$ for both data

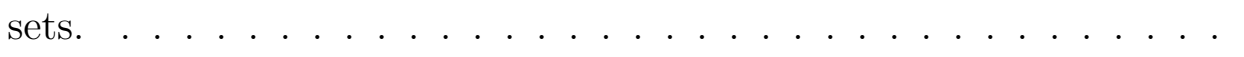

5.18 Average SB-corrected $\eta^{\prime}$ asymmetries for the nominal (black open circles with error bars) and altered (red filled circles) fit function. . . . .

5.19 The fit parameter $A$ of the altered fit function in each $t$ bin for the 0/90 peak region (blue circles) and side-band region (magenta squares), and for the 45/135 peak region (red crosses) and side-band region (green diagonal crosses). Fits to a constant function over each set of points

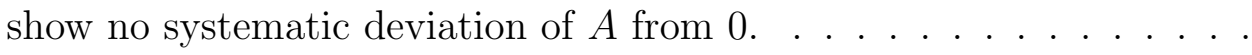

5.20 Means (points) and widths (vertical error bars) of the $\Sigma$ fit parameter distributions from fitting $500 \mathrm{MC}$ samples versus the number of bins used for the yield asymmetry histograms. For some points, the small vertical error bars are contained within the points. Figure from Reference [113]. . . . . . . . . . . . . . . . . .

B.1 $\eta \rightarrow \pi^{+} \pi^{-} \pi^{0} 0 / 90$ SB-corrected $\Sigma$ asymmetries for the studied bins of $t$ versus kinematic fit confidence level cut (left) and statistical errors

B.2 $\eta \rightarrow \pi^{+} \pi^{-} \pi^{0} 45 / 135$ SB-corrected $\Sigma$ asymmetries for the studied bins of $t$ versus kinematic fit confidence level cut (left) and statistical errors on those asymmetries (right).

B.3 $\eta \rightarrow 3 \pi^{0} 0 / 90$ SB-corrected $\Sigma$ asymmetries for the studied bins of $t$ versus kinematic fit confidence level cut (left) and statistical errors on those asymmetries (right). . . . . . . . . . . . . .

B.4 $\eta \rightarrow 3 \pi^{0} 45 / 135$ SB-corrected $\Sigma$ asymmetries for the studied bins of $t$ versus kinematic fit confidence level cut (left) and statistical errors on those asymmetries (right). . . . . . . . . . . . . .

B.5 $\eta \rightarrow 2 \gamma$ 0/90 SB-corrected $\Sigma$ asymmetries for the studied bins of $t$ versus kinematic fit confidence level cut (left) and statistical errors on those asymmetries (right). . . . . . . . . . . . . .

B.6 $\eta \rightarrow 2 \gamma$ 45/135 SB-corrected $\Sigma$ asymmetries for the studied bins of $t$ versus kinematic fit confidence level cut (left) and statistical errors on those asymmetries (right). . . . . . . . . . . . . 
B.7 $\eta^{\prime} \rightarrow \pi^{+} \pi^{-} \eta 0 / 90$ SB-corrected $\Sigma$ asymmetries for the studied bins of $t$ versus kinematic fit confidence level cut (left) and statistical errors on those asymmetries (right). . . . . . . . . . . . . . .

B.8 $\eta^{\prime} \rightarrow \pi^{+} \pi^{-} \eta 45 / 135$ SB-corrected $\Sigma$ asymmetries for the studied bins of $t$ versus kinematic fit confidence level cut (left) and statistical errors on those asymmetries (right). . . . . . . . . . . . . .

B.9 0/90 $\eta^{\prime}$ SB-corrected asymmetries for nominal (black open circles with error bars) and varied (coloured filled circles) cuts. Colours are as in the legend shown in Figure 5.12. . . . . . . . . . . . . . . . .

B.10 45/135 $\eta^{\prime}$ SB-corrected asymmetries for nominal (black open circles with error bars) and varied (coloured filled circles) cuts. Colours are as in the legend shown in Figure 5.12. . . . . . . . . . . . . .

B.11 0/90 $\eta^{\prime}$ side-band region asymmetries for nominal (black open circles with error bars) and varied (coloured filled circles) cuts. Colours are as in the legend shown in Figure 5.12. . . . . . . . . . . . . . . . .

B.12 45/135 $\eta^{\prime}$ peak region asymmetries for nominal (black open circles with error bars) and varied (coloured filled circles) cuts. Colours are as in the legend shown in Figure 5.12. . . . . . . . . . . . . . . .

B.13 45/135 $\eta^{\prime}$ side-band region asymmetries for nominal (black open circles with error bars) and varied (coloured filled circles) cuts. Colours are as in the legend shown in Figure 5.12. . . . . . . . . . . . 


\section{List of Tables}

1.1 Additive quantum number assignments for the six flavours of quarks.

1.2 $J^{P C}$ assignments for constituent quark model mesons with $L<2$. The physical states belonging to each multiplet and the names of each multiplet are listed. The mesons in each $J^{P C}$ group are distinguished from one another by their isospin. Mesons in the first column $\left(\pi, \rho, b_{1}\right.$, etc. $)$ have $I=1$, the second and third column contain the (possibly mixed) $I=0$ mesons, and the last column has the $I=1 / 2$ mesons. . . . . . .

1.3 A compilation of exotic quantum number hybrid approximate masses, widths, and decay predictions [12]. Masses are estimated from dynamical LQCD calculations with $m_{\pi}=396 \mathrm{MeV}$ [29]. The PSS (Page, Swanson and Szczepaniak) and IKP (Isgur, Kokoski and Paton) model widths are from [30], with the IKP calculation based on the model in $[24] \ldots \ldots \ldots \ldots \ldots \ldots \ldots$

3.1 Selection cuts on $\Delta t_{\mathrm{RF}}$ for various particles in the ST, TOF, BCAL, and FCAL. All times are in ns. . . . . . . . . . . . . .

3.2 The ranges in $t$ used to define each $t$ bin. The $t$-averaged results are those obtained using the entire range in $t$ spanned by the four bins. . 78

3.3 Integrated PS Flux and Flux Ratios for the two data sets. . . . . . . 81

4.1 Average asymmetries and statistical errors for the $\eta \rightarrow \pi^{+} \pi^{-} \pi^{0}$ decay channel. . . . . . . . . . . . . . . . . . . 93

4.2 Average asymmetries and statistical errors for the $\eta \rightarrow 3 \pi^{0}$ decay channel. 96

4.3 Average asymmetries and statistical errors for the $\eta \rightarrow 2 \gamma$ decay channel.101

4.4 Weighted averages and statistical errors of the three $\eta$ decay channels studied in this thesis. . . . . . . . . . . . . . . . . . . . 103

4.5 Average asymmetries, statistical errors, and systematic errors for the $\eta^{\prime} \rightarrow \pi^{+} \pi^{-} \eta$ decay channel. The sources of the systematic errors are

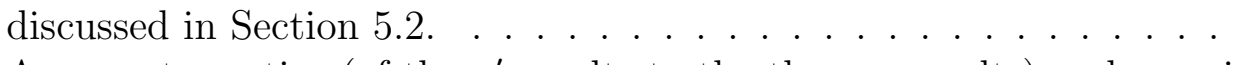

4.6 Asymmetry ratios (of the $\eta^{\prime}$ results to the three $\eta$ results) and associated statistical errors. . . . . . . . . . . . . . . . . . . . 111

4.7 Asymmetry ratios (of the $\eta^{\prime}$ results to the weighted average of the three $\eta$ results) and associated statistical errors. . . . . . . . . . . . 113

5.1 Systematic uncertainties for each bin of $t$ as found by Equation 5.1 on the average asymmetry. . . . . . . . . . . . . . . . 123 
5.2 Nominal, tighter, and looser cut variations for the study of systematic sensitivity of the $\Sigma$ asymmetry to event selection cuts. . . . . . . . 126

5.3 Systematic uncertainties for each bin of $t$ as found by Equation 5.2 on the average asymmetry. . . . . . . . . . . . . . . . . 128

5.4 Results of constant function fits to the nominal instrumental asymmetries for each data set and mass region. The fit parameter is consistent with 0 in each case. . . . . . . . . . . . . . .

5.5 Summary of systematic uncertainties assigned to the average $\eta^{\prime} \Sigma$ beam

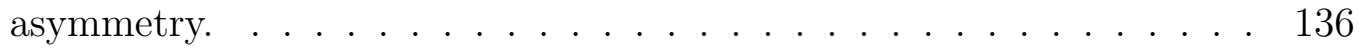

C.1 Asymmetries and statistical errors for the three $\eta$ decay channels separately and for the weighted average of the three $\eta$ results. . . . . . 155

C.2 Asymmetries, statistical errors, and systematic errors for the $\eta^{\prime}$ decay channel. . . . . . . . . . . . . . . . . . . . . . 155

C.3 Asymmetry ratios (of the $\eta^{\prime}$ results to the three $\eta$ results separately and to the weighted average of the three $\eta$ results) and associated statistical errors. . . . . . . . . . . . . . . . . . 155 


\title{
List of Abbreviations
}

\author{
BCAL Barrel Calorimeter \\ CDC Central Drift Chamber \\ CEBAF Continuous Electron Beam Accelerator Facility \\ CL Confidence Level \\ CP Charge Conjugation Parity \\ DIRC Detection of Internally Reflected Cherenkov Light \\ fADC Flash Analog-to-Digital Converter \\ FCAL Forward Calorimeter \\ FDC Forward Drift Chamber \\ JLab Thomas Jefferson National Accelerator Facility \\ JPAC Joint Physics Analysis Center \\ LH2 Liquid Hydrogen \\ LQCD Lattice Quantum Chromodynamics \\ MC Monte Carlo \\ MM Missing Mass \\ PDF Probability Density Function \\ PID Particle Identification \\ PMT Photomultiplier Tube \\ PS Pair Spectrometer \\ PWA Partial Wave Analysis \\ QCD Quantum Chromodynamics \\ QED Quantum Electrodynamics \\ RF Radiofrequency \\ SB Side-Band \\ SiPM Silicon Photomultiplier \\ SM Standard Model \\ ST Start Counter \\ TAGH Tagger Hodoscope \\ TAGM Tagger Microscope \\ TOF Time-of-Flight \\ TPOL Triplet Polarimeter
}




\section{Chapter 1}

\section{Introduction}

The GlueX experiment, based at the Thomas Jefferson National Accelerator Facility (JLab) in Newport News, Virginia, USA, aims to collect experimental evidence for the existence of exotic and hybrid mesons. These include mesons (quark-antiquark pairs $^{1}$ ) that feature contributions from excited gluons (the force-carrying boson of the strong interaction) and tetraquarks (particles comprising two quark-antiquark pairs). These particles are predicted to exist by Lattice QCD, a computational approach to studying Quantum Chromodynamics (QCD, the field theory of the strong interaction), and while some tantalizing evidence for the existence of certain hybrid and exotic states have been presented by other experiments in the past, GlueX is designed to produce these states at a relatively high rate up to invariant masses of 3.0 to $3.5 \mathrm{GeV} / c^{2}$, including the charmonium state $\mathrm{J} / \psi$. This should allow GlueX to perform the mapping of the low-lying meson spectrum, including hybrids and exotics, a field known as 'meson spectroscopy.'

A brief overview of QCD and Standard Model (SM) physics is given in this chapter, along with the existing evidence for hybrid or exotic states and the physics goals of GlueX, including the motivation for the work presented in this thesis on the $\Sigma$ beam asymmetry of the $\eta$ and $\eta^{\prime}$ mesons. Chapter 2 provides a description of the accelerator

\footnotetext{
${ }^{1}$ Throughout this thesis, the quark content of particles referred to are 'valence' quarks, those that contribute to the quantum numbers of the particle.
} 
facility at JLab and the GlueX detector, while Chapters 3 through 5 describe the data analysis, results, and systematic studies for the $\Sigma$ beam asymmetry analyses. Finally, Chapter 6 contains the conclusions and future directions of this line of research.

\subsection{The Standard Model}

The fundamental particles of matter, as they are known today, are divided into two main categories: fermions (particles with half-integer spin), some of which make up observable matter, and bosons (particles with integer spin), which mediate interactions between particles. 'Spin' here refers to a quantized intrinsic angular momentum, a principal quantum number of a particle. Each particle type has a unique set of quantum numbers, which act as identifiers. Other examples of identifying quantum numbers include electric charge, spin, isotopic spin, colour charge, and parity. Interactions between these fundamental particles are governed by four forces: the electromagnetic, the strong, the weak, and the gravitational forces. Of these, all but the gravitational force are part of the 'Standard Model' (SM) of particle physics.

Figure 1.1 shows the fundamental fermions and bosons in SM physics. The fermions are split into six quarks and six leptons, and further split into three 'generations.' The quarks are what make up 'hadrons,' like the proton, neutron, and pion. They interact with one another electromagnetically and through the strong interaction, which binds them together inside hadrons and nuclei. Leptons do not interact via the strong force, and so are not bound strongly together and can exist as free particles. The three generations are separated by large differences in mass, with most other properties being similar. For example, the generation I electron has a mass of $0.511 \mathrm{MeV}^{2}$, and the generation II muon has a mass of $105.658 \mathrm{MeV}$. The two particles behave identically in electromagnetic interactions, and the only real difference

\footnotetext{
${ }^{2}$ Throughout this thesis, natural units $(c=\hbar=1)$ are often used. Masses and momenta quoted in units of $\mathrm{GeV}$ are to be understood as being in units of $\mathrm{GeV} / c^{2}$ and $\mathrm{GeV} / c$, respectively.
} 


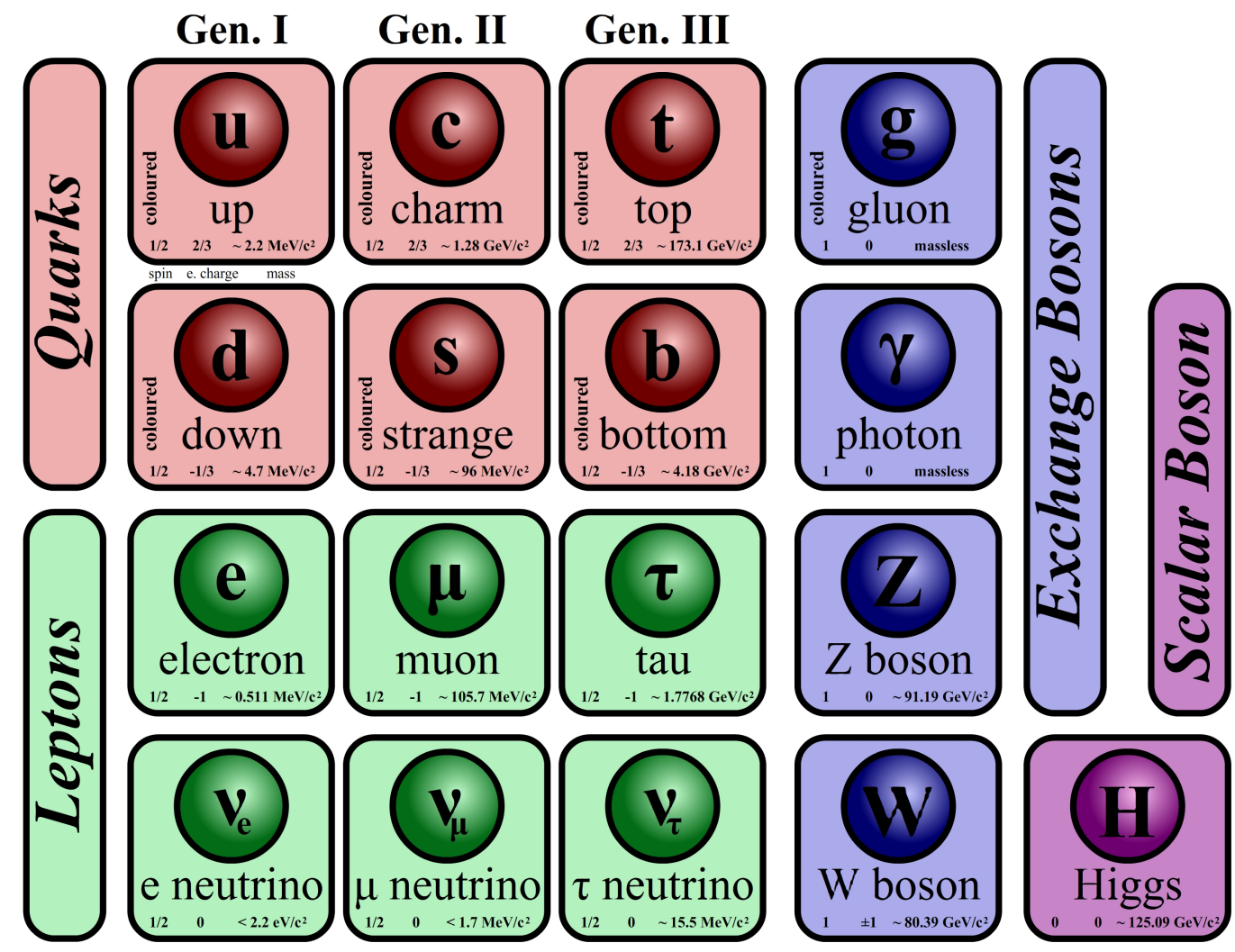

Figure 1.1: The fundamental particles of SM physics. Quarks and leptons are arranged by generation. Each particle's mass, spin, and electric charge are noted. Particles with colour charge are also indicated.

between them is the factor 200 increase in mass. Similarly, the generation III Tau has a mass of $1776.82 \mathrm{MeV}$, nearly twice the mass of the proton. Some differences can exist between generations other than mass, however, such as the isotopic spin of quarks (see Section 1.2). The spin 1 bosons are gauge bosons, mediators of the forces, meaning that the exchange of these particle between interacting particles are used to describe the observed fundamental forces. An observed repulsive or attractive interaction between two particles is described by one of the particles emitting a mediator boson (or 'exchange boson') and the other absorbing it. A mediator can only be produced or absorbed in an interaction by a particle that is sensitive to the mediator's force. For example, the electrically neutral photon is the exchange boson for the electromagnetic interaction, which is the force that governs interaction 
between electrically charged particles. An electrically neutral fundamental particle, then, cannot produce or absorb a photon. Finally, each particle has an 'antiparticle,' which has opposite charge (electric and/or colour) ${ }^{3}$.

The most familiar force is the electromagnetic force. Two particles with electric charge will either repel or attract each another depending on whether the charge on each is negative or positive. Each fundamental fermion in the SM has an electric charge, with leptons having integer multiples of the electron's charge and quarks having fractional multiples of the electron's charge (the hadrons that quarks comprise have integer charge). As mentioned, photons are the mediators of the electromagnetic force. The mathematical framework used to describe the electromagnetic exchange force is known as Quantum Electrodynamics (QED).

The strong force is described as the exchange of gluons between colour-charged objects. Of the fundamental fermions, only quarks have colour charge, but the gluon also carries colour charge. As such, the mathematical framework for describing the strong force, QCD, is quite different from QED (see Section 1.3).

The weak force acts on 'flavoured' particles, where flavour here refers to the type of a fundamental fermion. The exchange bosons of the weak interaction are the $\mathrm{W}$ (electrically charged) and Z (electrically neutral) bosons. The interaction is responsible for changing one flavour of particle into another, as in the decay of a neutron into a proton in which the emission of a $\mathrm{W}^{-}$boson changes a down quark in the neutron into an up quark. The mathematical framework describing this exchange force is Quantum Flavordynamics. However, at high energies, the weak and electromagnetic interactions behave similarly, prompting the development of Electroweak Theory, which is more commonly used to describe properties of the weak interaction.

The mediator of the interaction that gives particles mass, the 'Higgs mechanism,' is the Higgs boson. Particles that interact with the Higgs field have mass, while those

\footnotetext{
${ }^{3}$ Some particles are their own antiparticles.
} 
that do not are massless. The gravitational force, which describes how massive particles interact with one another, has no quantum mechanical mathematical description that can fit into the SM. The description of quantum gravity is left to physics beyond the SM.

\subsection{The Quark Model}

The constituent quark model, based on the work of Gell-Mann [1, 2], Ne'eman [3], and Zweig [4], aims to describe hadronic matter by way of quarks, subatomic particles that make up baryons (consisting of three quarks or antiquarks) and mesons (quarkantiquark pairs), collectively called hadrons. In order to describe known baryons, a model based on the $\mathrm{SU}(3)$ (special unitary group in three dimensions) symmetry group and three types of quarks was proposed. These three quarks, now called 'up,' 'down,' and 'strange' quarks, were assigned additive quantum numbers such that the combinations of quarks could describe the quantum numbers of the known baryons. In particular, this required each quark to possess a baryon number of $1 / 3$, along with fractional electric charge. By convention, quarks are fermions with positive parity. Antiquarks then have baryon number $-1 / 3$ and negative parity. This results in baryons (antibaryons) having a baryon number of $1(-1)$ and mesons having a baryon number of 0. Furthermore, the up and down quarks have isobaric spin (isospin) $I=1 / 2$ (the up quark having third component of isospin $I_{Z}$ or $I_{3}=1 / 2$, the down quark having -1/2) with the strange quark (and the three heavier quarks, the 'charm,' 'top,' and 'bottom') having an isospin of 0 . These properties are summarized in Table 1.1.

$\mathrm{SU}(3)$ is then used to determine the ways in which the three flavours of quarks can be combined into mesons and baryons. Meson multiplets are formed from a quark and an antiquark, and for the three light quarks, the possible combinations are grouped 


\begin{tabular}{|l||c|c|c|c|c|c|}
\hline Quantum Number & $d$ & $u$ & $s$ & $c$ & $b$ & $t$ \\
\hline \hline$Q$ (Electric Charge) & $-1 / 3$ & $2 / 3$ & $-1 / 3$ & $2 / 3$ & $-1 / 3$ & $2 / 3$ \\
$I$ (Isospin) & $1 / 2$ & $1 / 2$ & 0 & 0 & 0 & 0 \\
$I_{z}$ (Third Component of Isospin) & $-1 / 2$ & $1 / 2$ & 0 & 0 & 0 & 0 \\
$S$ (Strangeness) & 0 & 0 & -1 & 0 & 0 & 0 \\
$C$ (Charm) & 0 & 0 & 0 & 1 & 0 & 0 \\
$B$ (Bottomness) & 0 & 0 & 0 & 0 & -1 & 0 \\
$T$ (Topness) & 0 & 0 & 0 & 0 & 0 & 1 \\
\hline
\end{tabular}

Table 1.1: Additive quantum number assignments for the six flavours of quarks.

as $\mathbf{3} \otimes \overline{\mathbf{3}}=\mathbf{8} \oplus \mathbf{1}$, an octet (adjoint representation) and a singlet (trivial representation). There are three combinations of isospin $1\left(d \bar{u}, u \bar{d}\right.$, and $\left.\frac{1}{\sqrt{2}}(u \bar{u}-d \bar{d})\right)$, four combinations of isospin $1 / 2(d \bar{s}, u \bar{s}, s \bar{d}$, and $s \bar{u})$, and two combinations of isospin 0 $\left(|8\rangle=\frac{1}{\sqrt{6}}(u \bar{u}+d \bar{d}-2 s \bar{s})\right.$ and $\left.|1\rangle=\frac{1}{\sqrt{3}}(u \bar{u}+d \bar{d}+s \bar{s})\right)$. Because the $\mathrm{SU}(3)$ symmetry is not exact (owing to the higher mass of the strange quark compared to the up and down quarks), these isospin 0 (isoscalar) states are not necessarily the physical states. The observed states are mixtures of $|8\rangle$ and $|1\rangle$, defined by a mixing angle, $\vartheta[5]$.

$$
\left[\begin{array}{c}
f \\
f^{\prime}
\end{array}\right]=\left(\begin{array}{cc}
\cos \vartheta & \sin \vartheta \\
-\sin \vartheta & \cos \vartheta
\end{array}\right)\left[\begin{array}{c}
|8\rangle \\
|1\rangle
\end{array}\right]
$$

These nine states comprise a meson nonet, all with the same $J^{P C}$ quantum numbers (total angular momentum, parity, and charge conjugation quantum numbers). The mixing angle, $\vartheta$, is different for every $J^{P C}$ multiplet.

In the constituent quark model, a meson is a bound system of a quark and antiquark ( $q \bar{q}$ pair). Since both have a spin of $S=1 / 2$, the spin of the meson can then couple to either 0 or 1 . On top of that, there can be angular momentum, $L$, between the quark and antiquark. The total angular momentum, $J$, is then the coupling of $L$ and $S$. Using $J, L$, and $S$, the meson's $J^{P C}$ quantum numbers can be calculated [6].

The parity operator, which transforms a wavefunction $\psi(\vec{r})$ into $\psi(-\vec{r})$, has two eigenstates, 1 and -1 (or + and - ). The parity is a multiplicative quantity, such 
that the parity of a multiparticle state is equal to the product of the parities of the constituents. In order to relate the parity to the spin quantum numbers, the wavefunction is separated into a radial part and an angular part, as in Equation 1.2.

$$
P(\psi(\vec{r}))=P\left(R(r) Y_{l m}(\theta, \phi)\right)=R(r) Y_{l m}(\pi-\theta, \phi+\pi)=(-1)^{l} R(r) Y_{l m}(\theta, \phi)
$$

The parity operator then only operates on the angular part and introduces a factor of $(-1)^{L}$ into the parity of a meson. Since a meson has one quark (positive parity) and one antiquark (negative parity), the multiplicative nature of parity dictates an additional factor of -1 , so the parity of a meson becomes $P(q \bar{q})=(-1)^{L+1}[5]$.

The charge conjugation operator transforms a particle into its antiparticle. When applied to a meson state, the quark becomes an antiquark and vice-versa. Then, in order for the wavefunction to continue pointing to the quark, $\vec{r}$ transforms to $-\vec{r}$ in the same way as for the parity operator. Again, this introduces a factor of $(-1)^{L+1}$ to the charge conjugation quantum number. The operation also flips the spin wave function. This introduces a factor of $(-1)^{S+1}$, giving the charge conjugation operator the form $C(q \bar{q})=(-1)^{L+S}[5]$.

As an example, a $q \bar{q}$ pair with $L=0$ allows $J=0$ or 1 (since $S=0$ or 1 ). For $J=0, S=0$, so $P=-1$ (or - ), and $C=1\left(\right.$ or + ), giving $J^{P C}=0^{-+}$. For $J=1$, $S=1$, so $P=-1$, and $C=-1$, giving $J^{P C}=1^{--}$.

In this way, for various $L, J^{P C}$ quantum numbers for $q \bar{q}$ pairs allowable in the constituent quark model can be built up, and all quark model multiplets can be constructed. Table 1.2 shows the $J^{P C}$ multiplets for $L<2$, along with the corresponding physical state members of the multiplets and the name given to each group.

The model can be extended to SU(4) symmetry if the heavier charm quark is included, giving $q \bar{q}$ combinations grouped as $\mathbf{4} \otimes \overline{\mathbf{4}}=\mathbf{1 5} \oplus \mathbf{1}$. As with the strange quark, which is heavier than the up and down quarks, the charm quark is much 


\begin{tabular}{|c|c|c||c|c|c||cccc|c|}
\hline$L$ & $S$ & $J$ & $P$ & $C$ & $J^{P C}$ & \multicolumn{2}{|c|}{ Physical Mesons } & Group Name \\
\hline \hline 0 & 0 & 0 & - & + & $0^{-+}$ & $\pi$, & $\eta$, & $\eta^{\prime}$, & $K$ & Pseudoscalar \\
0 & 1 & 1 & - & - & $1^{--}$ & $\rho$, & $\omega$, & $\phi$, & $K^{*}$ & Vector \\
1 & 0 & 1 & + & - & $1^{+-}$ & $b_{1}$, & $h_{1}$, & $h_{1}^{\prime}$, & $K_{1}$ & Pseudovector \\
1 & 1 & 0 & + & + & $0^{++}$ & $a_{0}$, & $f_{0}$, & $f_{0}^{\prime}$, & $K_{0}^{*}$ & Scalar \\
1 & 1 & 1 & + & + & $1^{++}$ & $a_{1}$, & $f_{1}$, & $f_{1}^{\prime}$, & $K_{1}$ & Axial Vector \\
1 & 1 & 2 & + & + & $2^{++}$ & $a_{2}$, & $f_{2}$, & $f_{2}^{\prime}$, & $K_{2}^{*}$ & Tensor \\
\hline
\end{tabular}

Table 1.2: $\quad J^{P C}$ assignments for constituent quark model mesons with $L<2$. The physical states belonging to each multiplet and the names of each multiplet are listed. The mesons in each $J^{P C}$ group are distinguished from one another by their isospin. Mesons in the first column $\left(\pi, \rho, b_{1}\right.$, etc.) have $I=1$, the second and third column contain the (possibly mixed) $I=0$ mesons, and the last column has the $I=1 / 2$ mesons.

heavier than the $\mathrm{SU}(3)$ quarks, meaning that $\mathrm{SU}(4)$ symmetry is broken, but the basic multiplets can still be constructed. Weight diagrams are used to display these predictions in a succinct way. They contain the sixteen particles of SU(4) plotted as functions of isospin $I$, charm $C$, and hypercharge $Y=\mathcal{B}+S-\frac{C-B+T}{3}$ (here, $\mathcal{B}$ is baryon number, $S$ is the strangeness, $C$ is the charm, $B$ is the bottomness, and $T$ is the topness) [6]. The SU(4) weight diagrams for the pseudoscalar and vector mesons are shown in Figure 1.2(a) and (b).
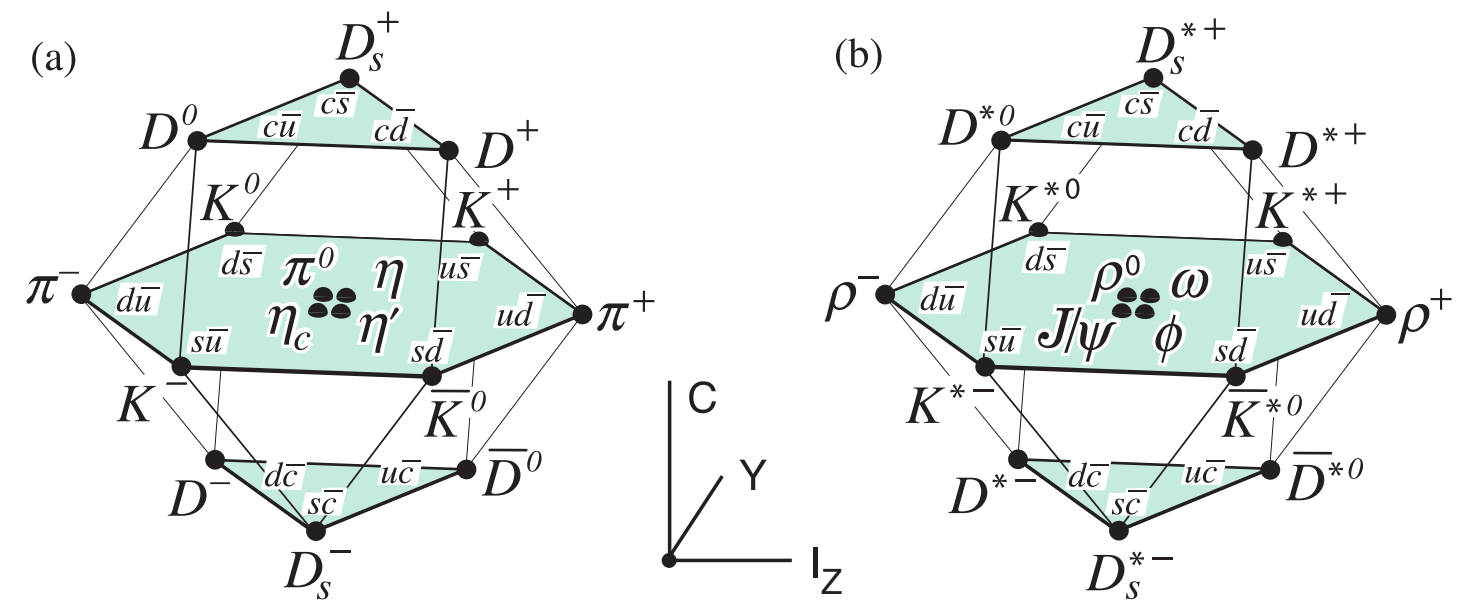

Figure 1.2: Weight diagrams for the pseudoscalar (a) and vector (b) mesons in $\mathrm{SU}(4)$. The light meson multiplets exist on the central plane $(C=0)$ along with the $c \bar{c}$ states, the $\eta_{C}$ and $J / \Psi$. Images from Reference [6]. 
Note that not all $J^{P C}$ combinations are allowed in this model. Exotic quantum numbers, meaning those that cannot be created by a simple quark and antiquark, include $J^{P C}$ combinations of $0^{--}, 0^{+-}, 1^{-+}, 2^{+-}, 3^{-+}$, and so on. Confirmation of a meson state with explicitly exotic quantum numbers could indicate a meson with contributions from an excited gluon (a gluon with angular momentum $L>0$ ), and confirmation of an entire exotic nonet would be a clear signal of the existence of exotic mesons.

\subsection{Exotic and Hybrid Mesons}

The quark model describes the quark content and organizes into multiplets many of the known mesons and baryons. However, it lacks dynamics, a description of how quarks interact and why hadrons are held together. This is done through QCD, a theory based on the extremely successful QED. In order to accommodate three identical quarks in a baryon (the $\Delta^{++}(u u u)$ or the $\Omega^{-}$(sss) baryons, for example), something the Pauli exclusion principle disallows ${ }^{4}$, an additional quantum number is introduced called 'colour charge.' This is analogous to electric charge in QED, but instead of two charges (positive and negative), there are three ('red,' 'blue,' and 'green'). A baryon, consisting of three quarks, then has one quark of each colour, and a meson, consisting of a quark and an antiquark, has a quark with one colour and an antiquark with the corresponding anticolour (antired, antiblue, or antigreen). In analogy with the additive colours of light, a baryon with one red, one blue, and one green quark (or a meson with one red quark and one antired quark) is 'white' or 'colour-neutral.' Quark confinement is the observed phenomenon that a quark cannot exist as a free particle, based on the lack of observation of free quarks (point-like

\footnotetext{
${ }^{4}$ The Pauli exclusion principle states that multiple spin $1 / 2$ particles cannot simultaneously occupy the same quantum state within a quantum system.
} 
objects with fractional electric charge). Quarks must then exist only inside colourneutral objects (hadrons).

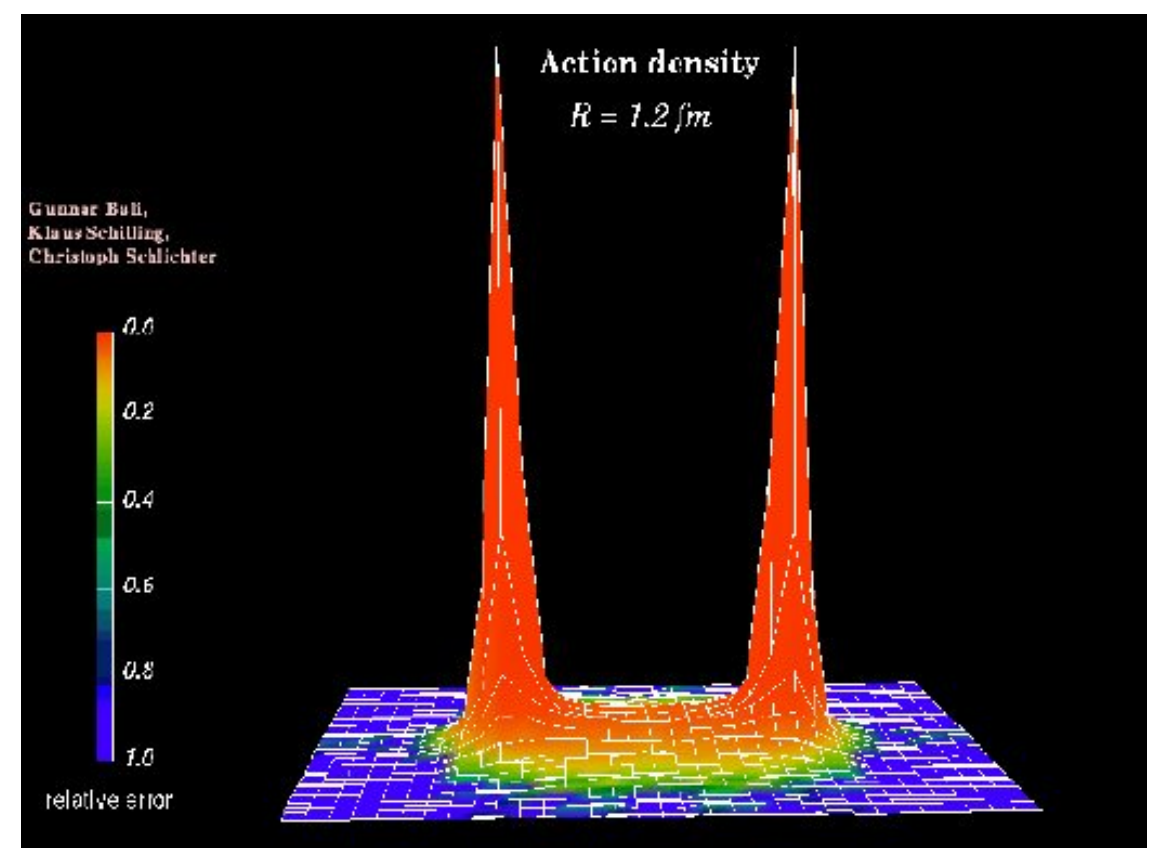

Figure 1.3: Lattice gauge theory simulation in $\mathrm{SU}(2)$. Shown is the action density $^{5}$ created by two static colour sources (infinitely heavy quarks, for example) separated by $1.2 \mathrm{fm}$ on a $32^{4}$ point lattice (see Section 1.3.1.2). Plot from Reference [8].

As with QED, QCD features an exchange boson that describes interactions between charged particles. In QED, this is the photon. Two electrically charged particles interacting is described as the exchange of a photon between the particles. In QCD, this exchange boson is the gluon, which is exchanged between quarks ${ }^{6}$. The gluons, unlike photons, carry colour charge of their own. As such, quarks can interact with quarks, and gluons can also interact with quarks and other gluons (a mechanism called self-interaction). In QED, lines of force between two charged particles are represented by virtual photons exchanged between the particles. In the same way, QCD represents lines of force by virtual gluons. However, since these gluons interact with one another, the lines of force are pulled together into a tight string, sometimes

\footnotetext{
${ }^{5}$ The action density is similar to the energy density [7].

${ }^{6}$ Exchange of mesons provides an effective description of the longer-range force between nucleons.
} 
called a 'flux tube,' as depicted in Figure 1.3, the lattice simulation snapshot from Bali, Schilling, and Schlichter [8]. The configuration helps explain quark confinement: as distance between two colour charges increases, the force remains constant and the energy stored in the flux tube increases.
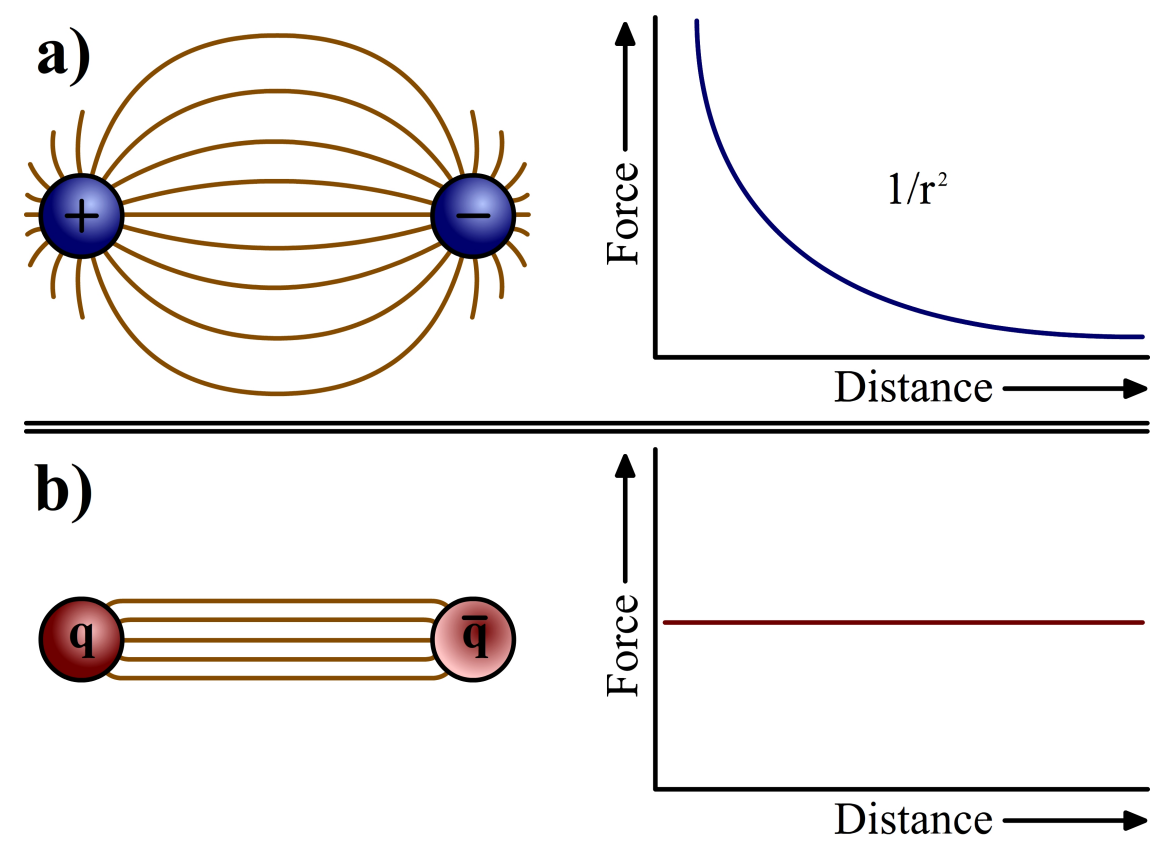

Figure 1.4: A sketch showing the field lines and force between two charge sources in electrodynamics (a) and QCD (b).

This is in contrast to QED, where the force lines are such that increased distance causes a decrease of force with an inverse squared law (see Figure 1.4). Then, if two colour charges are separated a great distance, the energy stored in the flux tube is large enough to pass the quark pair production threshold, resulting in a new pair of quarks, each one coupling to one of the original quarks (shown graphically in Figure 1.5). The gluon-gluon interaction allows for a much more complex system where different types of particles are possible, including 'normal' colour-neutral objects with gluonic degrees of freedom (hybrids) and pure-gluon states (glueballs). 


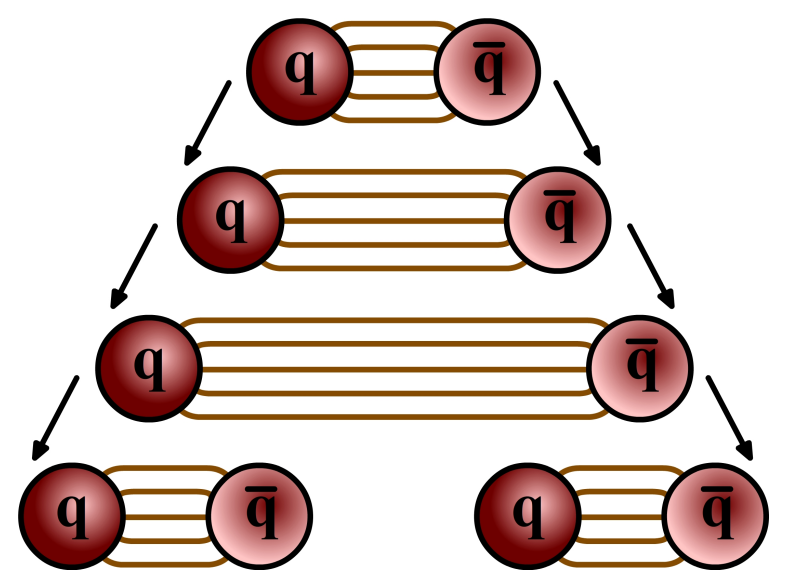

Figure 1.5: A sketch depicting the production of a pair of quarks. Rather than liberating a quark from a hadron, the energy in the flux tube becomes large enough with increasing distance to generate a new $q \bar{q}$ pair, resulting in two distinct hadrons.

\subsubsection{Models and Predictions for Exotics and Hybrids}

In the high-energy regime of quark interactions, the separation distance between quarks is very small, meaning that the energy stored in the flux tube is also small (represented graphically as a flux tube of small density), and the binding energy between quarks becomes negligible. This is 'asymptotic freedom,' and it allows accurate perturbative calculations of QCD processes at high energies using the known QCD Lagrangian (Equation 1.3) [6].

$$
\mathcal{L}=\sum_{q} \bar{\psi}_{q, a}\left(i \gamma^{\mu} \partial_{\mu} \delta_{a b}-g_{s} \gamma^{\mu} t_{a b}^{C} A_{\mu}^{C}-m_{q} \delta_{a b}\right) \psi_{q, b}-\frac{1}{4} F_{\mu \nu}^{A} F^{A \mu \nu}
$$

The $\gamma^{\mu}$ are Dirac $\gamma$-matrices ${ }^{7}$, the $\psi_{q, a}$ are quark field spinors for quarks of flavour $q\left(\right.$ mass $\left.m_{q}\right)$ with colour $a . q$ can be one of six flavours, and $a$ one of three colours $\left(N_{c}=3\right) . A_{\mu}^{C}$ are gluon fields, where $C$ runs from 1 to $N_{c}^{2}-1=8$. In other words, there are 8 kinds of gluons. The $t_{a b}^{C}$ are the 8 generators of the $\mathrm{SU}(3)$ group. They appear in the Lagrangian term that describes the coupling of quark and gluon fields (the second term in Equation 1.3) and serves to rotate the quark's colour in SU(3)

\footnotetext{
${ }^{7}$ Everywhere in the Lagrangian, a Greek letter index implies a four-component object (three spatial dimensions and one temporal dimension).
} 
space upon interaction with the gluon. $g_{s}$ is the QCD coupling constant, and $F_{\mu \nu}^{A}$ is the QCD field tensor, given by Equation $1.4[6]$.

$$
F_{\mu \nu}^{A}=\partial_{\mu} A_{\nu}^{A}-\partial_{\nu} A_{\mu}^{A}-g_{s} f_{A B C} A_{\mu}^{B} A_{\nu}^{C} \quad \text { and } \quad\left[t^{A}, t^{B}\right]=i f_{A B C} t^{C}
$$

Here, $f_{A B C}$ are the structure constants of the $\mathrm{SU}(3)$ group. The field tensor of QCD is similar to the electromagnetic field tensor with the addition of the third term in Equation 1.4. This term arises from the gluon self-interaction (gluons interacting with other gluons). The third term in Equation 1.3 simply describes free quarks at rest. The first and third terms together form the Dirac equation that quarks, similarly to electrons in QED, obey.

However, at lower energies, perturbative calculations become complicated or impossible, owing to a large strong coupling constant and a non-perturbative vacuum [9]. So, effective theories, models, or computer calculations are used to predict interactions and bound states. The most commonly referred to models are briefly introduced in this section.

\subsubsection{Constituent Gluon Model}

Many forms of this type of model exist, but in general, the model builds up $J^{P C}$ quantum numbers in the same way as the constituent quark model but allowing for pure-gluon states and hybrids by assuming a gluon $J^{P}$ of $1^{-}$. The gluon can possess angular momentum, $l_{g}$. The parity of a hybrid meson $(q \bar{q} g)$, for example, includes a factor of -1 (from the intrinsic gluon parity) and a factor of $(-1)^{l_{g}}$. A hybrid's parity is then $P(q \bar{q} g)=(-1)^{L+l_{g}}$, where $L$ is the orbital angular momentum between the quark and antiquark, as before. The charge conjugation of a hybrid gains a factor of -1 as well, giving $C(q \bar{q} g)=(-1)^{L+S+1}[10]$. In this model, most $J^{P C}$ combinations can be realized for a hybrid meson.

Similarly, 2- or 3-gluon states can be built (glueballs). The low angular momentum 
states are those with $J^{P C}$ of $0^{++}, 0^{-+}$, and $2^{++}(2$-gluon $)$, or $0^{-+}, 1^{--}$, and $3^{--}(3-$ gluon). Along with these models, an effective potential model can be used to predict masses of glueball and hybrid multiplets for comparison to other theories or lattice QCD calculations $[10,11]$.

\subsubsection{Lattice QCD}

Lattice QCD (LQCD) is a non-perturbative approach to solving QCD: it is a lattice gauge theory formulated on a grid or lattice of points in space and time. It provides a tool for calculating interactions in the low energy (non-perturbative) regime. Quark fields reside on the lattice points, separated evenly in space and time by a distance $a$, and gluon fields act as links between lattice points. The continuum theory, true QCD, is then found by taking the limit as the lattice spacing vanishes. Because of early ${ }^{8}$ limitations in computing power, compromises were required, introducing systematic errors on predictions made with LQCD. One simplification is the removal of vacuum polarization effects, known as 'quenching.' Larger lattice spacing and fewer lattice sites also help with computing power, and often, unphysically large quark masses are used to ease computation requirements. These all make extrapolation down to expected quark masses and vanishing lattice spacing more difficult, resulting in large systematic errors.

Over the last few decades, computing power has grown exponentially. Processing speeds have increased dramatically, and the cost to operate large, fast clusters of computers to do lattice calculations has likewise decreased. With modern computational power, calculations on the lattice can be done without quenching on massive lattices, with ever smaller lattice spacings and quark masses, leading to theoretical results that are in fair agreement with experimental results, where available [6, 12].

A number of input parameters are required to do LQCD calculations, including

\footnotetext{
${ }^{8}$ Around the year 2000 .
} 
the lattice spacing $a$, the quark masses, and the $\mathrm{CP}$ (charge conjugation parity) violating phase $\theta . \theta$ allows $\mathrm{CP}$ violation (a difference in physics between matter and anti-matter), but is usually set to 0 for lattice calculations. The quark masses are set from experimental data. Heavy quark (quarks other than $u, d$, and $s$ ) masses can be tuned by setting mesons with heavy quark content to their experimental values, and light quark ( $u, d$, and $s$ quarks) masses are tuned by making certain light-quark meson mass ratios match experiment [6]. The precision of the tuning is usually quoted as the lattice $\pi^{0}$ mass, which is generally around $400 \mathrm{MeV}$ for modern lattice calculations ${ }^{9}$. Once these input parameters have been specified, the masses or resonance properties of all other states can be predicted, including normal hadrons, hybrids with gluonic degrees of freedom, and glueballs $[13,14,17]$. Lattice results on some multiquark states (tetraquarks and pentaquarks) also exist $[18,19]$. Modern results are generally in agreement with experimental data, and many predictions exist for as-yet undiscovered particles including exotics and hybrids of various types.

\subsubsection{Flux Tube Model}

A useful alternative to full lattice QCD is the flux tube model. In short, it takes the idea of the lattice and substitutes the gluon field links with a string. For large spacing between two colour sources, a flux tube can be thought of as a string with tension. Excitations of the gluon field are then described by vibrations of that string. The notion of modelling this kind of system with vibrating strings dates back to Nambu, one of the founders of string theory [20]. Some early models of effective string theories include those in References [21], [22], and [23]. The lattice string is approximated as having a number of 'mass beads' along its length corresponding to the number of lattice spacings the connected quarks are apart from one another. The beads' masses are related to the tension in the string and the lattice spacing, $a$, the beads

\footnotetext{
${ }^{9}$ This is in comparison with the physical mass of the $\pi$ of about $135 \mathrm{MeV}$.
} 
interact with a linear potential, and they are allowed to oscillate about their resting positions. The angular momentum contribution of an excited gluon is modelled by a string phonon ${ }^{10}$, which contributes to the total angular momentum of a particle system. With flux tube and flux tube decay models [24], quantities such as masses of particles, quantum numbers, and decay rates can be calculated and simulated. For example, such studies predict the lightest hybrid masses to be between 1.8 and $1.9 \mathrm{GeV}[12,25]$. A good number of simplifications and approximations are applied in such a model, but they tend to show good agreement with lattice calculations and experimental results $[12,26]$. They provide a simpler picture of states and decays in situations where rigorous calculation or simulation is unnecessary or not possible, and before major improvements with lattice calculations that have come along recently, flux tube models were the most trusted calculations for decay rates and resonance properties available.

One of the results from flux tube calculations of vibrational hybrid meson $(q \bar{q} g$ with gluonic excitations) decays is that the prominent decays are to excited mesons, $S+P$ (one $L=0$ and one $L=1$ meson), for example [27, 28]. This type of observation provides experimenters some insight into what decay channels may be fruitful for searching for particular $J^{P C}$ hybrids.

\subsubsection{Exotic Hybrid Meson Predictions}

All modern models predict the lightest hybrid meson to be a $J^{P C}=1^{-+}$particle in the mass range of 1.5 to $2.1 \mathrm{GeV}$ [10]. In the past, much experimental attention has been paid to this mass region and quantum number combination, and some evidence has been seen of hybrid signals (see Section 1.4). Now, with recent LQCD results [14], an extremely rich spectrum of mesons is predicted. The lattice QCD spectrum in Figure 1.6 shows the light meson spectrum $(u, d$, and $s$ quark contributions) for

\footnotetext{
${ }^{10} \mathrm{~A}$ phonon is a quantum of vibrational mechanical energy.
} 
a lattice size of $24^{3}$ spatial and 128 temporal lattice points with a pion mass of $391 \mathrm{MeV}$. The low-lying hadrons (light pseudoscalars and vectors) are identifiable with the approximately correct masses and octet/singlet mixing angles. The three right-most columns hold exotic quantum number states. Sets of three states (two isoscalar and one isovector) correspond to a meson nonet (the $I=1 / 2$ states are not shown). The lightest hybrid supermultiplet, which is a set of four $J^{P C}$ hybrid nonets $\left(0^{-+}, 1^{-+}, 1^{--}\right.$, and $\left.2^{-+}\right)$corresponds to the states marked in orange. Experimental verification of all states in the explicitly exotic $1^{-+}$nonet, along with evidence of the other nonets in the supermultiplet, is a necessary step for claiming the existence of mesons with gluonic degrees of freedom. Similar lattice spectra exist for mesons in the charm quark sector [15], for baryons [16], and for glueballs [17].

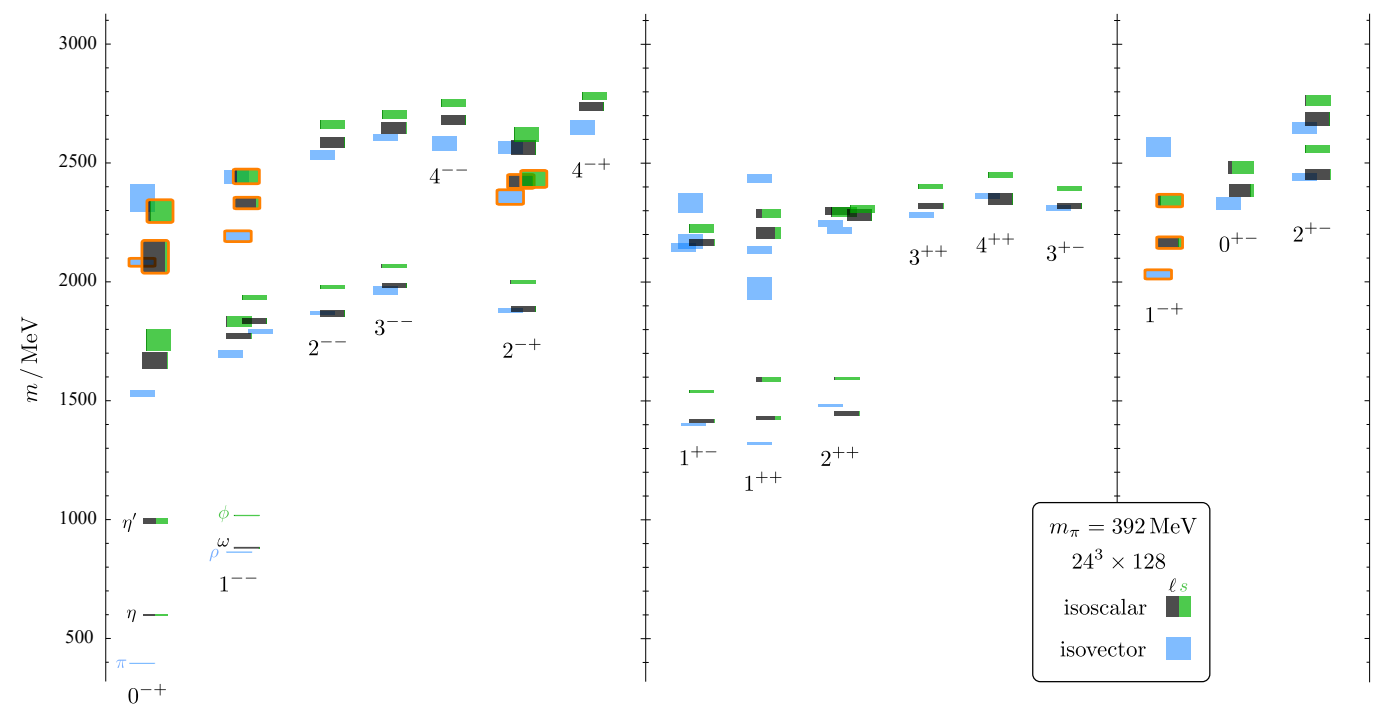

Figure 1.6: The LQCD meson spectrum, displayed as state mass versus quantum numbers $\left(J^{P C}\right)$. The height of a state indicates the error on the mass. Octet/singlet mixing angle is indicated by black and green contributions to isoscalar mesons. Candidates for the lightest hybrid supermultiplet are highlighted in orange [14] (colour online).

Table 1.3 summarizes calculations with LQCD and various models for low-mass hybrid mesons with explicitly exotic quantum numbers. Some allowed decay modes 
(found simply through quantum number counting arguments) for each of these particles are also listed. From this summary, a short list of final states with a small number of stable particles can be made, which may provide the best opportunities to search for evidence of an exotic signal.

\begin{tabular}{ccccc}
\hline \hline \multirow{2}{*}{ Name } & $J^{P C}$ & Total Width MeV & \\
& & PSS & IKP & Allowed Decay Modes \\
\hline$\pi_{1}$ & $1^{-+}$ & $81-168$ & 117 & $b_{1} \pi, \pi \rho, \pi f_{1}, \pi \eta, \pi \eta^{\prime}, \eta a_{1}, \pi \eta(1295)$ \\
$\eta_{1}$ & $1^{-+}$ & $59-158$ & 107 & $\pi a_{1}, \pi a_{2}, \eta f_{1}, \eta f_{2}, \pi \pi(1300), \eta \eta^{\prime}, K K_{1}^{A}, K K_{1}^{B}$ \\
$\eta_{1}^{\prime}$ & $1^{-+}$ & $95-216$ & 172 & $K K_{1}^{B}, K K_{1}^{A}, K K^{*}, \eta \eta^{\prime}$ \\
\hline$b_{0}$ & $0^{+-}$ & $247-429$ & 665 & $\pi \pi(1300), \pi h_{1}, \rho f_{1}, \eta b_{1}$ \\
$h_{0}$ & $0^{+-}$ & $59-262$ & 94 & $\pi b_{1}, \eta h_{1}, K K(1460)$ \\
$h_{0}^{\prime}$ & $0^{+-}$ & $259-490$ & 426 & $K K(1460), K K_{1}^{A}, \eta h_{1}$ \\
\hline$b_{2}$ & $2^{+-}$ & $5-11$ & 248 & $\pi a_{1}, \pi a_{2}, \pi h_{1}, \eta \rho, \eta b_{1}, \rho f_{1}$ \\
$h_{2}$ & $2^{+-}$ & $4-12$ & 166 & $\pi \rho, \pi b_{1}, \eta \omega, \omega b_{1}$ \\
$h_{2}^{\prime}$ & $2^{+-}$ & $5-18$ & 79 & $K K_{1}^{B}, K K_{1}^{A}, K K_{2}^{*}, \eta h_{1}$ \\
\hline \hline
\end{tabular}

Table 1.3: A compilation of exotic quantum number hybrid approximate masses, widths, and decay predictions [12]. Masses are estimated from dynamical LQCD calculations with $m_{\pi}=396 \mathrm{MeV}$ [29]. The PSS (Page, Swanson and Szczepaniak) and IKP (Isgur, Kokoski and Paton) model widths are from [30], with the IKP calculation based on the model in [24].

\subsection{Existing Evidence for Non-Quark Model Mesons}

Mesons are arranged into multiplets with various $J^{P C}$ quantum numbers, and the light meson states that have been experimentally verified fit the framework of the constituent quark model quite cleanly with one exception. There is an overabundance of light scalar $\left(J^{P}=0^{+}\right)$states claimed in experiments. Scalar candidates include the $\kappa(800), \sigma(500)^{11}, f_{0}(980), a_{0}(980), f_{0}(1370), K_{0}^{*}(1430), a_{0}(1450)$, $f_{0}(1500)$, and $f_{0}(1710)$, along with a number of unconfirmed resonances like the $f_{0}(1790)$ and $f_{0}(1810)[6]$. Usually, the lightest scalar nonet is interpreted as the

\footnotetext{
${ }^{11}$ The $\sigma(500)$ is sometimes referred to as the $f_{0}(500)$ and most commonly referred to as the $[\pi \pi]_{S}$ (two-pion S-wave).
} 
$\kappa, f_{0}(980)$, and $a_{0}(980)$, and constituent quark models can accommodate up to three more scalar nonets in this mass range [28]. It is expected, then, that if all these candidates and unconfirmed resonances are actual meson resonances, at least some of them should be non-conventional mesons. Lattice calculations show the lightest scalar glueball to lie around 1.5 to $1.7 \mathrm{GeV}[17]$, meaning one or more of the isoscalar $f_{0}$ particles may be pure glue states, or some may be superpositions of mass eigenstates (contributions from glueball and conventional meson states). In addition, interpretations of the $f_{0}(980)$ and $a_{0}(980)$ (as well as some other light scalars) as tetraquarks or meson molecules are not uncommon [6,31,32], though solid evidence of any of these ideas has not been presented.

As for explicitly exotic mesons, evidence for a few $J^{P C}=1^{-+}$states exists. Above $1 \mathrm{GeV}$ or so in mass, the spectrum of mesons becomes quite crowded, to the point where resonances are often not visible in simple mass spectra. Here, Partial Wave Analysis (PWA) is useful to disentangle contributions from different resonances. A final state spectrum can be decomposed into contributions from different produced resonances decaying through various decay channels. For example, the $3 \pi$ final state is modelled as some created resonance decaying to a pion and an isobar with angular momentum $L$ between them. The isobar then decays to the other two pions ${ }^{12}$ (see Figure 1.7). The isobar can be any meson that decays to two pions such that it could have come from the decay of the resonance with a specified $J^{P C}$. The resonance quantum numbers (partial waves) corresponding to the possible $3 \pi$ isobar decay channels then forms the wave set used in the partial wave analysis. The contribution from each partial wave to the total cross section (a measure of the probability of a particular final state being produced by a particular initial state reaction) can be calculated individually, then the ensemble is fit to angular distributions obtained in data. The result is partial wave amplitudes that represent the relative contributions of each

\footnotetext{
${ }^{12} \mathrm{~A}$ variety of corrections are made to the basic isobar model to account for problems related to unitarity, analyticity, and crossing symmetry [33].
} 
partial wave to the final state system [34].

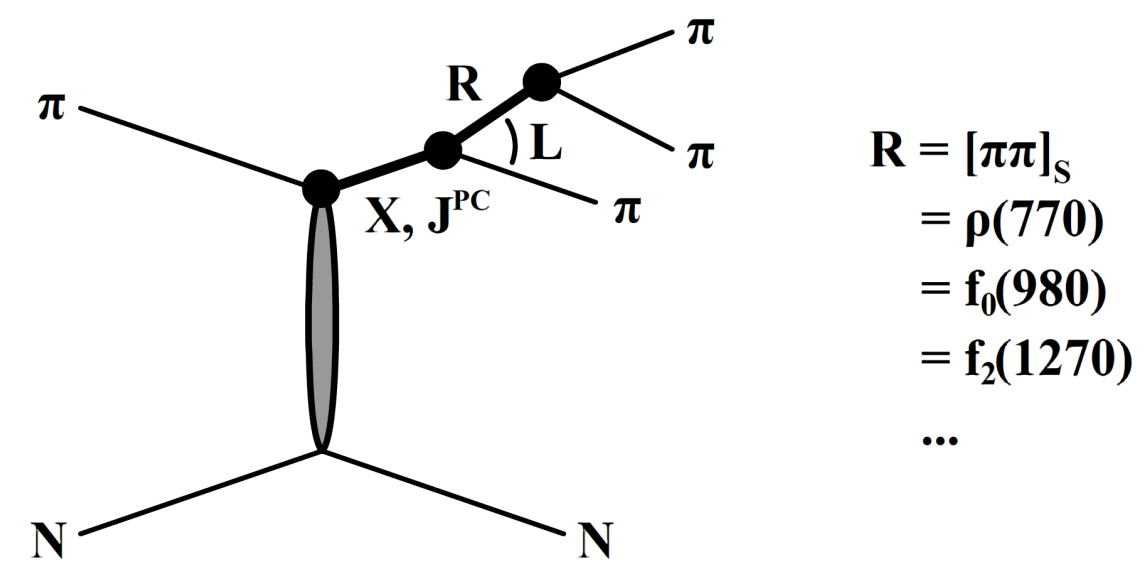

Figure 1.7: An example of the isobar model. Creation of a three-pion resonance (X, with quantum numbers $J^{P C}$ ) is modelled by creation of a pion and an isobar $(\mathrm{R})$ with the isobar subsequently decaying to two pions. The total cross section is the sum of all possible contributions.

Small contributions from suppressed partial waves can be detected in this way, even when peaks in the mass spectra are impossible to see. However, it is extremely important that the wave set is comprehensive. A full partial wave analysis would require an infinite amount of waves, but the set is often limited to partial waves with $J<7$ and $L<7$ or so, since waves beyond this are generally negligible and computation resources are limited [35]. Waves which are forbidden by kinematics and structureless waves with low relative intensities are excluded. Evidence for exotic particles comes from performing such a partial wave analysis, then re-performing the same analysis but excluding the exotic partial wave contributions. A lower fit probability in the latter implies a significant contribution from the exotic wave.

The $\pi_{1}(1600)$ has been suggested by PWA in diffractive production ${ }^{13}$ using a pion beam, in $p \bar{p}$ annihilation, and in $c \bar{c}$ meson $\left(\chi_{c 1}\right)$ decay by $\operatorname{VES}[36,37,38]$, E852 [39, 40, 41, 42, 43], COMPASS [44, 45, 46], CLEO-c[47], and the Crystal Barrel

\footnotetext{
${ }^{13}$ Diffractive production is characterized by a reaction during which no quantum numbers are exchanged between the beam and target particles.
} 
experiment [48]. Though not all observations between different collaborations are consistent, particularly signals in $3 \pi$ decay modes ${ }^{14}$ [51], the seemingly robust signals extracted in the $b_{1} \pi, f_{1} \pi$, and $\eta^{\prime} \pi$ decay modes from different experiments give confidence to the existence of a real exotic state. Comparisons of the branching ratios of the $\pi_{1}(1600)$ obtained from the analysis of the VES experiment are in reasonable agreement, with decays to a $P+S$ wave system more prominent than a system of two spin 0 particles [12].

The second explicitly exotic meson is the $\pi_{1}(2015)$. So far proposed only by the E852 Collaboration $[42,43]$, this state decays to $b_{1} \pi$ and $f_{1} \pi$, as expected of a hybrid meson, but without further confirmation from other experiments, nothing much can be said about this state. A third state, the $\pi_{1}(1400)$, was also proposed by the E852 Collaboration, and while other collaborations have seen evidence of such a state, it's resonant nature is unclear $[52,53]$. Coupled with the fact that it has only been seen in one decay channel (which is not expected of a hybrid meson [12]), it is unlikely to be a $q \bar{q} g$ object.

Non-exotic hybrid candidates have been reported, including the $\pi(1800)\left(0^{-+}\right)[54]$ and the $\eta_{2}(1870)\left(2^{-+}\right)$[55]. A study performed by Donnachie and Kalashnikova [56] suggests the existence of $1^{--}$hybrids as well. A number of meson molecules and possible tetraquarks that involve heavy quarks $(X, Y$, and $Z$ particles that don't fit predictions of quark models) have also been confirmed [6].

Sophisticated lattice calculations show a vibrant spectrum of non-conventional mesons, and confirmation of members of the lightest hybrid supermultiplet would all but prove the existence of mesons with contributions from gluonic degrees of freedom. New experiments, like PANDA, FAIR, and GlueX, will be integral in searching for conclusive evidence of these and other hybrid states.

\footnotetext{
${ }^{14}$ No evidence for the $\pi_{1}(1600)$ is seen at all in the $3 \pi$ channel in the E01-017 photoproduction experiment [49] at CLAS [50].
} 


\subsection{Exotic Production with the GlueX Experiment}

Mapping the meson spectrum, particularly the hybrid multiplets, is the main goal of the GlueX experiment. GlueX is a fixed-target, linearly-polarized photon beam experiment that focuses on the production and detection of hybrid resonances. The high-energy $(9 \mathrm{GeV})$ photon beam and high resolution for detecting both neutral and charged particles (see Chapter 2) allows meson spectroscopy of states up to about $3.0-3.5 \mathrm{GeV}$ in mass. Detection of the explicitly exotic hybrid multiplets is of particular interest. Lattice and model calculations suggest that these exotic states tend to exist as $q \bar{q}$ pairs with gluonic excitations $(q \bar{q} g)$, where the spins of the quarks are aligned [57], which motivates the use of a photon beam for GlueX.

Much of the evidence for exotic hybrid mesons (the $\pi_{1}(1600)$ and possibly the $\left.\pi_{1}(2015)\right)$ has come from experiments with pion beams. In an interaction where the target nucleon recoils, the quantum numbers of a produced resonance is dependent on the quantum numbers of the beam particle. For a pion (or kaon) probe, $S=0$, meaning that to get an exotic hybrid meson with aligned spins, there must be a spin-flip transition. A photon probe, however, carries the quantum numbers of a vector meson $(S=1)$, meaning that photoproduction allows exotic hybrids directly. In addition, estimates suggest that hybrids should be produced at roughly the same rate as conventional mesons in photoproduction [58].

The polarization of the photon beam provides the means to distinguish between naturalities of exchanged particles. Photoproduction of a particle occurs through the exchange of a virtual meson ${ }^{15}$ between the beam photon and the target nucleon (a proton, in the case of GlueX). This can be done through 'natural' or 'unnatural' parity exchange ${ }^{16}$. Natural parity exchange corresponds to exchange of a meson with

\footnotetext{
${ }^{15}$ For photoproduction of the pseudoscalar $\eta$ and $\eta^{\prime}$ mesons, which is the subject of this thesis, a virtual vector meson is exchanged.

${ }^{16}$ The naturality of a particle is determined by how it behaves under parity transformations. Natural parity particles have $P=(-1)^{J}$, and unnatural parity particles have $P=(-1)^{J+1}$.
} 
quantum numbers $J^{P}=0^{+}, 1^{-}, 2^{+}$, etc., while unnatural parity exchange corresponds to $J^{P}=0^{-}, 1^{+}, 2^{-}$, etc. The differential cross section (cross section as a function of a particular variable, most often solid angle or interaction energy) for photons polarized perpendicular to the reaction plane is dominated by natural parity exchange and the differential cross section for photons polarized parallel to the reaction plane is dominated by unnatural parity exchange, to leading order in energy [59]. Thus, the polarized beam used for the GlueX experiment allows measurement of exchange naturalities, which provides information about the production mechanism of measured resonances. If the production mechanism is already known, the beam polarization can be used to filter resonances by naturality.

\subsection{Current GlueX Efforts}

To carry out a PWA, GlueX must be able to reconstruct pure samples of the final state mesons $\left(\pi, \eta, \eta^{\prime}, \rho, \omega, \phi\right.$, etc. $\left.{ }^{17}\right)$ and known resonances $\left(a_{0}, f_{0}, b_{1}\right.$, etc.). In particular, the $\eta$ and $\eta^{\prime}$ mesons are critical, since they feature heavily in many expected exotic meson decay channels (see Table 1.3). Simulating the experiment accurately is also important, since a detailed Monte Carlo (MC) simulation that matches what is seen in data allows for the calculation of detector acceptance and efficiency, which is required for producing cross sections and doing PWA. As such, the first steps toward the main goals at GlueX include studying simple mesons and known resonances while improving matching between simulation and data for simple processes.

Before the high-luminosity, low-emittance photon beam experiments at Jefferson Lab, existing photoproduction results came mainly from SLAC (the Stanford Linear Accelerator Center), most of which were centered on cross section measurements.

\footnotetext{
${ }^{17}$ Low-lying mesons with simple decays can be considered final-state particles for the purposes of PWA.
} 
A major focus of current GlueX analyses is to increase photoproduction statistics at GlueX energies of about $9 \mathrm{GeV}$ and to measure quantities like the $\Sigma$ beam asymmetry of particles. In addition, the first direct photoproduction signal of the $\mathrm{J} / \psi$ particle near threshold has recently been seen by GlueX [60]. Studies of the cross section as a function of incoming photon energy helps in studying heavy quark states, which may be explained by either two- or three-gluon exchanges [61], so studies of this particle at $9 \mathrm{GeV}$ are of interest. Continual improvements to the GlueX simulation software are made to enable cross section analysis and PWA, and improvements to the reconstruction software are pursued to produce higher-purity, higher-resolution signals. Until this work is complete, much effort is being put toward the study of the $\Sigma$ beam asymmetry for a number of particles.

The beam asymmetry is a polarization observable that gives insight into the exchange mechanism's naturality. It is accessible in data through the polarized photon beam without the need for detector acceptance correction, as discussed more thoroughly in Sections 1.6.1 and 3.2. The $\Sigma$ beam asymmetry of the $\rho^{0}, \pi^{0}$ and $\eta$ (decaying to 2 photons) at $9 \mathrm{GeV}$ have been published by GlueX $[62,63]$, and analysis of the $\pi^{+}, \pi^{-}$, and $a_{0}$ are underway. Preliminary $\Sigma$ beam asymmetry results for the $\eta$ (in

decay channels other than to two photons) and $\eta^{\prime}$ have been produced, and are the subject of this thesis.

\subsubsection{Pseudoscalar Beam Asymmetry}

Along with the beam asymmetry, $\Sigma$, there are fifteen other polarization observables that arise from various polarization combinations. Experiments can be done under conditions of a polarized beam, polarized target, and a polarized recoil baryon. Experiments with different combinations of these have access to different polarization observables. The single-polarization observables are obtainable with one of the three constituents polarized. A polarized beam with an unpolarized target, as in GlueX, 
can yield the beam asymmetry, $\Sigma$. Polarized target experiments can yield the target asymmetry, $T$, and polarized recoil experiments can yield the recoil asymmetry, $P$. Then, for each two-polarization combination (beam-target, target-recoil, or beamrecoil), four double-polarization observables can be measured. The unpolarized cross section, $\sigma_{0}$, is the sixteenth observable. Each of these observables is related to an element in the expression for the total cross section of an interaction, along with the related beam, target, and recoil polarization vectors $\left(\overrightarrow{P^{S}}, \overrightarrow{P^{i}}\right.$, and $\overrightarrow{P^{b}}$, respectively) as in Equation 1.5 [64].

$$
\begin{aligned}
\sigma & =\sigma_{0}\left[\left(1+P_{x}^{S} P_{y}^{i} P_{y}^{b}\right)+P\left(P_{y}^{b}+P_{x}^{S} P_{y}^{i}\right)+\Sigma\left(P_{x}^{S}+P_{y}^{i} P_{y}^{b}\right)+T\left(P_{y}^{i}+P_{x}^{S} P_{y}^{b}\right)\right. \\
& +E\left(P_{z}^{S} P_{z}^{i}+P_{y}^{S} P_{x}^{i} P_{y}^{b}\right)+F\left(P_{z}^{S} P_{x}^{i}-P_{y}^{S} P_{z}^{i} P_{y}^{b}\right)+G\left(-P_{y}^{S} P_{z}^{i}+P_{x}^{S} P_{x}^{i} P_{y}^{b}\right) \\
& +H\left(-P_{y}^{S} P_{x}^{i}-P_{z}^{S} P_{z}^{i} P_{y}^{b}\right)+C_{x}\left(P_{z}^{S} P_{x}^{b}+P_{y}^{S} P_{y}^{i} P_{z}^{b}\right)+C_{z}\left(P_{z}^{S} P_{z}^{b}-P_{y}^{S} P_{y}^{i} P_{x}^{b}\right) \\
& +O_{x}\left(-P_{y}^{S} P_{x}^{b}+P_{z}^{S} P_{y}^{i} P_{z}^{b}\right)+O_{z}\left(-P_{y}^{S} P_{z}^{b}-P_{z}^{S} P_{y}^{i} P_{x}^{b}\right)+T_{x}\left(P_{x}^{i} P_{x}^{b}+P_{x}^{S} P_{z}^{i} P_{z}^{b}\right) \\
& \left.+T_{z}\left(P_{x}^{i} P_{z}^{b}-P_{x}^{S} P_{z}^{i} P_{x}^{b}\right)+L_{x}\left(P_{z}^{i} P_{x}^{b}-P_{x}^{S} P_{x}^{i} P_{z}^{b}\right)+L_{z}\left(P_{z}^{i} P_{z}^{b}-P_{x}^{S} P_{x}^{i} P_{x}^{b}\right)\right]
\end{aligned}
$$

For a linearly-polarized photon beam at an angle $\Phi$ with respect to the reaction plane, the polarization vector is $\vec{\epsilon}=(\cos (\Phi)$, $\sin (\Phi), 0)$, leading to a pure photon state (in the circular polarization basis, $|+\rangle$ and $|-\rangle$ ) $|\Phi\rangle=\frac{-1}{\sqrt{2}}\left[e^{-i \Phi}|+\rangle-e^{i \Phi}|-\rangle\right]$. Then, the density matrix for the photon is

$$
\rho_{\gamma}^{\text {lin. pol. }}(\Phi)=|\Phi\rangle\langle\Phi|=\frac{1}{2}\left(\begin{array}{cc}
1 & -e^{-2 i \Phi} \\
-e^{2 i \Phi} & 1
\end{array}\right)
$$

At GlueX, a partially polarized beam is achieved, where $P_{\gamma}$ is the degree of polarization. The density matrix is then a mixture of the pure state from Equation 1.6 and pure $| \pm\rangle$ states, as in Equation 1.7 [65].

$$
\rho_{\gamma}(\Phi)=\frac{1-P_{\gamma}}{2}(|+\rangle\langle+|+|-\rangle\langle-|)+P_{\gamma}|\Phi\rangle\langle\Phi|=\frac{1}{2}\left(I+\overrightarrow{P^{S}}(\Phi) \cdot \vec{\sigma}\right)
$$


Here, $\vec{\sigma}$ are the Pauli spin matrices and $\overrightarrow{P^{S}}(\Phi)=-P_{\gamma}(\cos (2 \Phi), \sin (2 \Phi), 0)$. Since only the beam is polarized for the GlueX experiment, $\overrightarrow{P^{i}}$ and $\overrightarrow{P^{b}}$ are both zero. Then, the polarized cross section for pseudoscalar production can be written in terms of the unpolarized cross section (from Equation 1.5) as

$$
\sigma_{\text {pol. }}=\sigma_{0}\left(1+\Sigma P_{x}^{S}\right)=\sigma_{0}\left(1-P_{\gamma} \Sigma \cos (2 \Phi)\right)
$$

In an experiment, $\Sigma$ can be extracted by fixing the polarization angle of the photon beam, $\phi_{\gamma}$, then measuring the angle of the reaction plane (by detecting the produced pseudoscalar and/or recoil baryon), $\phi$. The difference between these two angles is $\Phi=\phi-\phi_{\gamma} . \Sigma$ is defined such that natural parity exchange (meson production plane perpendicular to the beam polarization, as discussed in Section 1.5) will contribute positively and unnatural parity exchange (meson production plane parallel to the beam polarization) will contribute negatively [65], as captured in Equation 1.9.

$$
\Sigma=\frac{\sigma_{\text {nat. }}-\sigma_{\text {unnat. }}}{\sigma_{\text {nat. }}+\sigma_{\text {unnat. }}}
$$

As an example, if the polarization angle of the photon beam was set to $\phi_{\gamma}=0^{\circ}$, and a pseudoscalar was produced with purely natural parity exchange, it is expected that enhancements in data yield should be seen at perpendicular angles to the polarization angle, $90^{\circ}$ and $270^{\circ}$. In this case, $\Phi=\phi$, so the $\cos (2 \Phi)$ dependence in the cross section implies that the yield will have a sinusoidal behaviour with minima at $\Phi=0^{\circ}$ and $180^{\circ}$ and maxima at $90^{\circ}$ and $270^{\circ}$, in agreement with the concept of natural parity exchange.

Each polarization observable is defined by some combination of 'reaction amplitudes,' which describe spin state transitions between the beam, target, and recoil baryon [64]. In order to do a 'complete' experiment, meaning one that uniquely defines the reaction amplitudes of the interaction, a number of these polarization 
observables must be measured for a particular energy [66]. Another experiment at Jefferson Lab, CLAS, was previously run with a polarized photon beam at lower energies than GlueX, and data from this run preiod has recently been used to measure some single- and double-polarization observables for photoproduction of a number of particles $[67,68,69,70,71,72]$. GlueX is only sensitive to $\Sigma$, but since this has not been measured for photoproduction of most particles at GlueX energies, particularly the $\eta$ and $\eta^{\prime}$, and because $\Sigma$ data helps theorists constrain exchange models, this is an interesting observable to study.

\subsubsection{Predictions of $\eta$ and $\eta^{\prime}$ Beam Asymmetries}

A number of models ${ }^{18}$ have been developed over the past few decades to describe light meson photoproduction data. With the recent availability of large data sets from GlueX and complimentary experiments, these models can be tested to see if they hold at higher beam energies, if they need adjustments, or if new models must be developed. To this end, the recently published GlueX results on the $\Sigma$ beam asymmetry of the $\pi^{0}$ and $\eta$ [63], shown in Figure 1.8, feature previous $\pi^{0}$ data from SLAC and comparisons to model predictions for energies similar to the GlueX beam energy ${ }^{19}$. Since the beam asymmetry is generally dependent on the squared fourmomentum transfer to the target, the Mandelstam variable $t=\left(P_{\text {target }}-P_{\text {recoil }}\right)^{2}$, model predictions are given as functions of $t$ and data are analyzed in bins of $t$ for comparison.

The new $\pi^{0}$ data from GlueX do not show the same trends as the data from SLAC, though the SLAC data was produced over a large range of beam energies (from 4 to $18 \mathrm{GeV})$, the average of which $(10 \mathrm{GeV})$ is similar to the GlueX beam's energy, so the differences in the results may be reasonable. The GlueX $\pi^{0}$ results do agree,

\footnotetext{
${ }^{18}$ These models are based on calculations in Regge theory [73], which are beyond the scope of this thesis.

${ }^{19}$ Details regarding these models can be found in the listed references in the caption of Figure 1.8 and in Reference [63].
} 

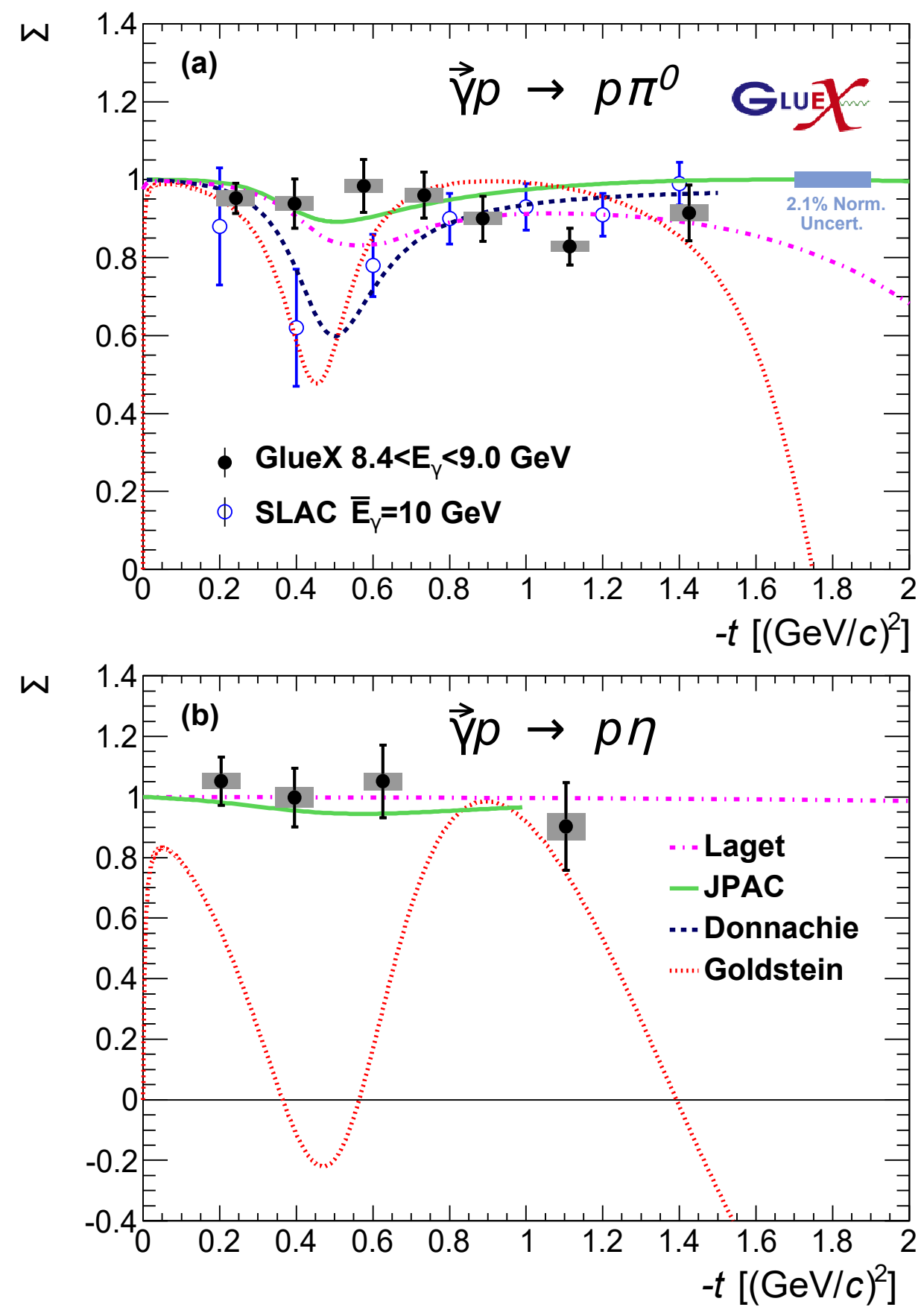

Figure 1.8: GlueX $\Sigma$ beam asymmetry for photoproduction of the $\pi^{0}$ (a) and the $\eta$ (b) off the proton at $E_{\gamma}=9 \mathrm{GeV}$ (black filled circles) [63]. Uncorrelated systematic errors are indicated by grey boxes and combined statistical and systematic uncertainties are given by black error bars. SLAC results for the $\pi^{0}$ for an average beam energy of $10 \mathrm{GeV}$ are also shown [74] (blue open circles), along with various model predictions (Laget [75, 76], JPAC [77, 78], Donnachie [79], and Goldstein [80]) (colour online). 
however, with the most recent model predictions from the Joint Physics Analysis Center (JPAC), as do the GlueX $\eta$ results.

JPAC has also released model predictions for the $\Sigma$ beam asymmetry of the $\eta^{\prime}$ [59]. The beam-target exchange for $\eta$ and $\eta^{\prime}$ photoproduction is dominated by the $\omega, \rho$, $\phi, b, h$, and $h^{\prime}$ vector mesons. In terms of naturality, exchange of the $\omega, \rho$, and $\phi$ $\left(J^{P}=1^{-}\right)$is natural parity exchange, while the exchange of the $b, h$, and $h^{\prime}\left(J^{P}=1^{+}\right)$ is unnatural parity exchange. If there is no contribution from hidden-strangeness exchange of $\phi$ and $h^{\prime}$ mesons, it is expected that the beam asymmetries for the $\eta$ and $\eta^{\prime}$ should be equal [59]. The model from JPAC predicts the beam asymmetry ratio between the $\eta^{\prime}$ and the $\eta$ allowing for hidden-strangeness exchange.

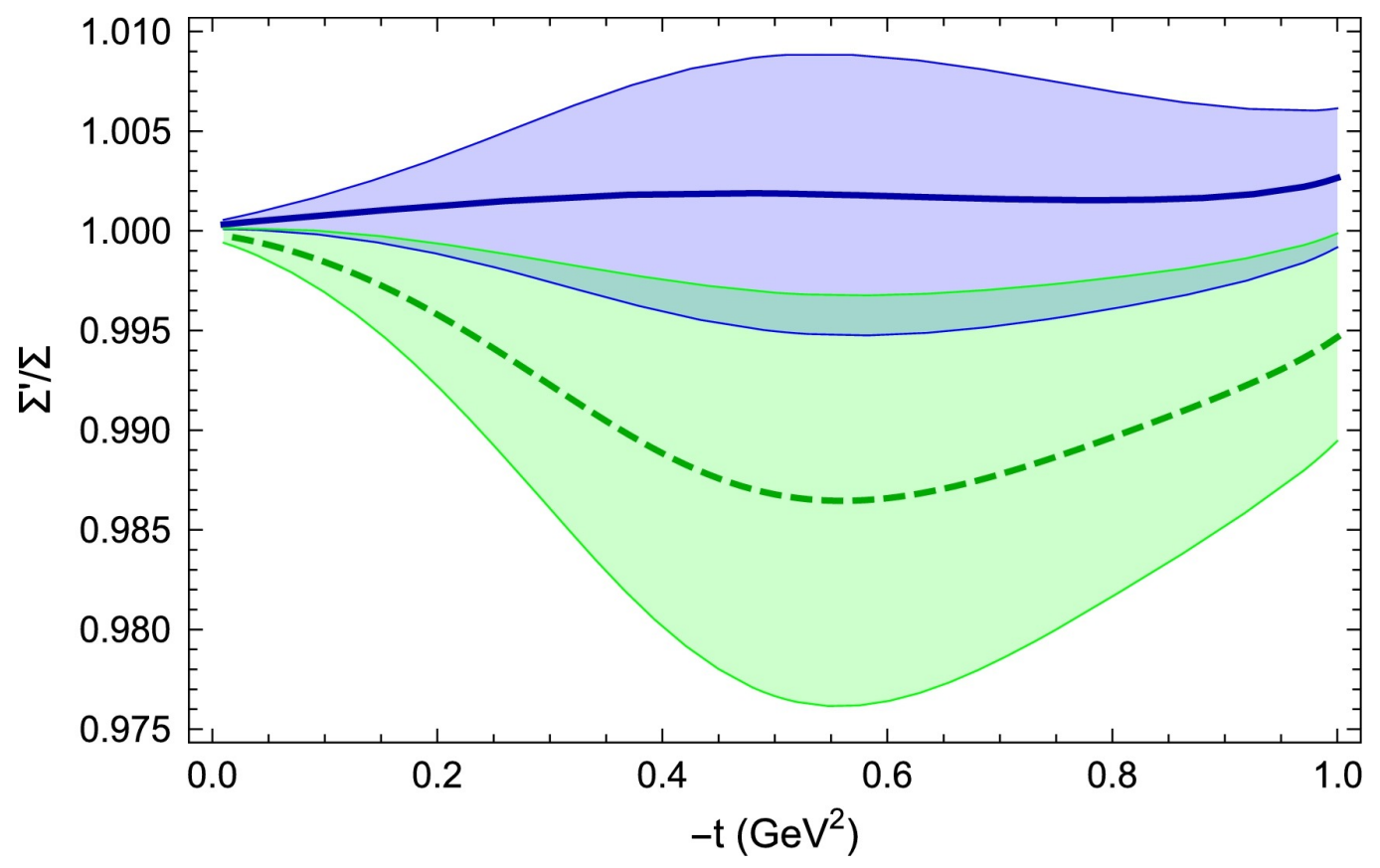

Figure 1.9: Theory curves produced by JPAC for $\Sigma_{\eta^{\prime}} / \Sigma_{\eta}$. The two curves derive from two different model assumptions for the axial vector radiative decay strengths, with the similarly coloured bands representing the $\pm 1 \sigma$ error on each curve. More detailed information regarding the model can be found in the published JPAC paper [59] (colour online).

Input parameters of the model come from data for radiative decays of the mesons involved in the beam-target exchange, where available. Assumptions are made for the 
radiative decays of the mesons with no data (in particular, the axial vector decays, $b \rightarrow \eta^{(\prime)} \gamma$ and $\left.h \rightarrow \eta^{(\prime)} \gamma\right)$. First, it is assumed that the $b$ and $h$ couple identically to the nucleon, since it is difficult to distinguish empirically between $b$ and $h$ exchanges. Then, it is assumed that the axial vector exchanges follow the same pattern as the vector exchanges. Two possibilities arise: the axial vector coupling strengths are assumed to be equal to the $\rho$ strengths, or equal to the $\omega$ strengths. The two prediction curves generated under these two possibilities are shown in Figure 1.9. These assumptions are on the extreme ends of what is believed to be reasonable [59]. The model suggests that the $\eta^{\prime}$ beam asymmetry should be nearly identical to the $\eta$ beam asymmetry, with the ratio of the two beginning to increase at high $-t^{20}$. A significant deviation from a ratio of 1 in data might imply non-negligible hidden-strangeness contribution from the $\phi$ or $h^{\prime}$ exchange, or that the photoproduced $\eta$ or $\eta^{\prime}$ is not totally decoupled from the target.

The $\Sigma$ beam asymmetries of the $\eta$ and $\eta^{\prime}$ that are presented in this thesis are compared to these model predictions and the previous $\eta$ GlueX measurements in Chapter 4 .

\footnotetext{
${ }^{20}$ Throughout this thesis, 'high $-t$ ' means larger $|t|$. For ease of viewing, trends are plotted versus $-t$ in all figures, so low magnitudes are closer to the y-axis and large magnitudes are increasing to the right of the $y$-axis.
} 


\section{Chapter 2}

\section{The GlueX Experiment}

The GlueX Experiment is conducted at JLab, home to CEBAF, the Continuous Electron Beam Accelerator Facility, which produces high-frequency bursts of electrons accelerated through superconducting electromagnetic cavities. The accelerator can provide high energy electron beams to four different experimental halls simultaneously. The recently constructed and commissioned fourth hall, Hall D, houses the GlueX detector ${ }^{1}$. A thin diamond wafer is used to convert the Hall D electron beam into a polarized photon beam, which is transported to the hall. The GlueX detector is a nearly- $4 \pi$ hermetic detector, designed to be sensitive to all final-state particles produced from the interaction of the beam photon with a proton in the target. In this chapter, brief overviews of each of the major components of the beam line and detector are presented.

\subsection{CEBAF at Jefferson Lab}

The electron beam at Jefferson Lab is generated through the photoelectric effect by shining a high frequency laser onto a Gallium Arsenide photocathode. Electron bursts that are produced are then shaped into evenly-spaced beam bunches (pulses), which

\footnotetext{
${ }^{1}$ Construction of Hall D began in May, 2009, and the first beam was delivered to Hall D in May, 2014.
} 
are accelerated with superconducting radiofrequency (RF) multi-cell cavities [81]. These cavities are shaped vacuum chambers, which, when supplied with RF power, produce standing electromagnetic waves (modes). The Transverse Magnetic (TM) mode that is used as the fundamental accelerating mode in these cavities is the $\mathrm{TM}_{010}$ mode $[82]^{2}$. TM modes are well-suited for acceleration of charged particles because the electric field is directed along one axis and the magnetic field is everywhere perpendicular to that axis, so no magnetic beam deflections are possible. The three indices in the mode assignment, $\mathrm{TM}_{m n p}$, indicate the distribution of the electric and magnetic fields in the three cylindrical coordinates, $\phi, r$, and $z . m$ is the number of full-period variations of the fields in azimuth, $n$ is the number of nodes in the radial variation of the longitudinal field (the electric field, in the case of TM modes) minus 1 , and $p$ is the number of half-period variations of the fields in the longitudinal direction [83]. Thus, the $\mathrm{TM}_{010}$ mode (along with all $\mathrm{TM}_{0 n 0}$ modes) provides fields that are azimuthally symmetric and constant along the longitudinal direction, with the electric field strongest at $r=0$ and no magnetic field at $r=0$. Particles are thus directed through the cavities along the $\mathrm{r}=0$ axis.

The direction of the electric field switches between parallel and anti-parallel with the beam at a regular interval defined by the supplied RF frequency. The speed of the electron bunches in the beam must be coupled with the frequency of the beam and matched to the frequency of the RF cavity, such that the electromagnetic mode provides an accelerating kick at times when the electron beam bunches pass through the cavity, and a decelerating kick in the times when no beam bunch is in the cavity. At CEBAF, the niobium cavities are designed with an accelerating frequency of $1497 \mathrm{MHz}$, so the electron beam must also be produced with this frequency. Rather than using one $1497 \mathrm{MHz}$ laser, however, four lower frequency lasers are used on the same photocathode simultaneously. This is to facilitate four-Hall operation, meaning

\footnotetext{
${ }^{2}$ The $\mathrm{TM}_{010}$ mode is a resonance mode in a perfect pillbox cavity. In a shaped multi-cell cavity, the field is distorted.
} 
delivery of four distinct electron beams to all four experimental halls at CEBAF. The four beams are interlaced, such that all four beams together create a string of electron beam bunches at $1497 \mathrm{MHz}$. Two lasers pulsing at $499 \mathrm{MHz}$ and two lasers pulsing at $249.5 \mathrm{MHz}$, appropriately spaced in phase, are used. Then, RF separators throughout the accelerator, operating at the same frequencies, pick out every third (499 MHz) or sixth $(249.5 \mathrm{MHz})$ beam bunch for steering. In this way, all four beams can be treated as one $1497 \mathrm{MHz}$ beam for acceleration, then separated for delivery to different halls.

In order to achieve variable energy and different energies for each of the four beams, the accelerator is constructed in a racetrack-like configuration, shown in Figure 2.1. Electrons from the injector are accelerated through the first set of 25 cryomodules, which house the accelerating cavities, then enter arcing and focusing beam components that bring them to the second set of 25 cryomodules. After this second set, beams can be delivered to experimental Halls A, B, or C, or one (or more) of them can be directed into another arcing section that leads back to the first set of cryomodules for additional acceleration. Additional arcing beam lines exist to circulate the beams for up to five passes through the accelerating sections, with each pass providing $2.2 \mathrm{GeV}$ in electron energy. Beams are referred to by the number of passes through the whole 'racetrack,' making a 5-pass beam the maximum for Halls A, B, and C. After five passes, one final arcing section brings the beam back to the first set of cryomodules for a final acceleration towards Hall D, making the Hall D beam 5.5-pass. The Hall D beam line is designed to operate only in 5.5-pass mode, and gives Hall D a maximum electron beam energy of $\sim 12 \mathrm{GeV}$.

Before the construction of Hall D, each of three $499 \mathrm{MHz}$ beams were delivered to one of Halls A, B, and C. To simultaneously deliver beam to Hall D, additional RF separators and another injector laser were added [84]. Effectively, the frequency of one of the pre-existing three beams is lowered to $249.5 \mathrm{MHz}$ so that every second 


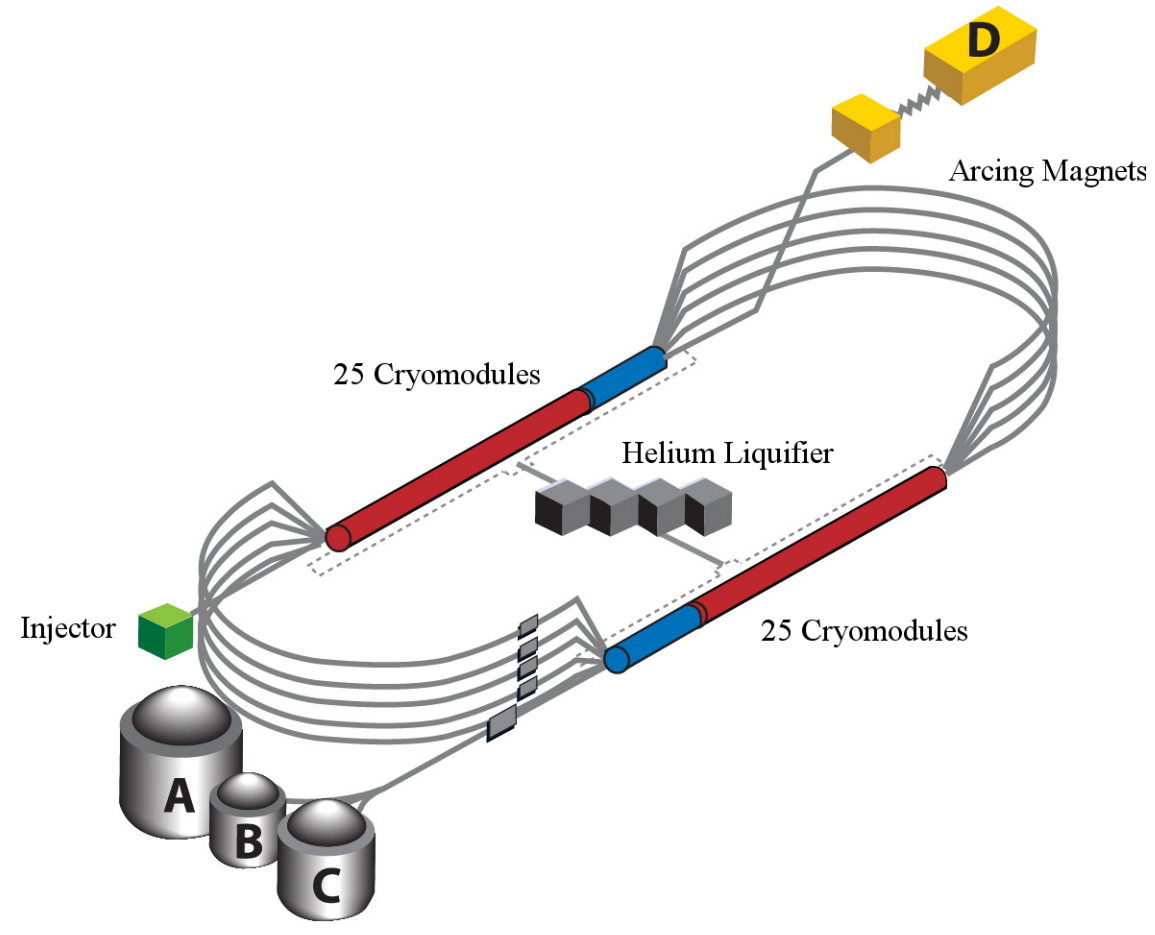

Figure 2.1: CEBAF at Jefferson Lab. A high-frequency electron beam is accelerated to high energy and supplied to up to four experimental halls simultaneously.

beam bunch in that sequence can be delivered to Hall D. Since Hall D requires the highest energy achievable in order to accomplish its physics mission of looking for exotic states of matter up to $\sim 3.5 \mathrm{GeV} / c^{2}$ in mass, it will always be operated in 5.5-pass mode, meaning that it is the highest-pass beam between Halls A, B, and C which must suffer decreased frequency.

In terms of energy, the original 40 cryomodules were able to produce a maximum electron beam energy of $\sim 6 \mathrm{GeV}$. The recent upgrade to the facility ${ }^{3}$ added ten new higher gradient cryomodules that pushed the maximum electron beam energy up to $\sim 12 \mathrm{GeV}$. This upgrade in energy allows for higher center-of-mass energies for production experiments, a finer resolution for probing nucleon substructure, and represents many other significant upgrades in terms of discovery potential for experiments operating out of Jefferson Lab.

\footnotetext{
${ }^{3}$ During the same time as the construction of Hall D.
} 


\subsection{Hall D}

The goal of GlueX is to search for evidence of exotic and hybrid mesons using partial wave analysis, which requires accurate reconstruction of the final state particles. Thus, the detector must be capable of detecting both charged and neutral particles and must be able to determine the energy and momentum of each type of particle. In addition, the experiment must be simulated accurately in order to determine the efficiency of the detection and reconstruction, and the lightest, simplest mesons and baryons must be well-understood so clean samples can be selected for analysis of more complex decay topologies. The GlueX detector is composed of many subsystems, each of which contribute specific information toward identifying a particle or its properties, and all of which are important for getting an accurate representation of what decaying resonances might be produced in an interaction.

\subsubsection{Beam Line}

\subsubsection{Polarized Photon Beam}

The electron beam coming into Hall $\mathrm{D}$ is converted into a photon beam in the Tagger Hall. This is done through the process of coherent Bremsstrahlung on a thin diamond wafer. The incident electron can interact with the Coulomb field surrounding a nucleus and produce a high-energy photon, where the energy of the photon is related to the outgoing angle of the photon with respect to the incident beam. In turn, these are related to the momentum transferred to the struck nucleus, $\vec{q}$. A smooth continuum of $\vec{q}$ values contribute to the Bremsstrahlung process on a single nucleus and the effects are averaged, giving an effect called 'incoherent Bremsstrahlung.' However, when an entire crystal recoils from the incident electron all at once, called 'coherent Bremsstrahlung,' the $\vec{q}$ values that contribute to the resulting photon energy 
and angular spectra are constrained to be reciprocal lattice vectors ${ }^{4}$, which are nearly perpendicular to the incident beam direction [85]. By orienting the diamond wafer in a particular way, a small number of these reciprocal lattice vectors can be picked out. The resulting spectra are then composed of only a few distinct angles and energies. The orientation of the diamond can be adjusted further so that only the lowest-energy photon peak dominates the spectra.

In addition, the photon created by the Bremsstrahlung process is partially linearly polarized ${ }^{5}$ depending on the energies involved and the outgoing photon angle [86]. Coherent Bremsstrahlung from an oriented diamond wafer thus generates a monochromatic, partially polarized photon beam from an incident electron beam. The outgoing electrons are swept away from the beam line by a magnetic field into the Tagger Hodoscope (TAGH) and Tagger Microscope (TAGM). These subsystems are used to measure the energy of the outgoing electron (see Section 2.2.1.2), which allows the calculation of the Bremsstrahlung photon energy. However, the electron beam emittance (divergence at the target), mosaic spread of the crystal (imperfections in lattice sites), and multiple scattering of electrons in the crystal smear the outgoing angles and energies of the Bremsstrahlung photons [87].

In order to minimize the effects of mosaic spread and multiple scattering, and to preserve the beam polarization, the GlueX Experiment requires a thin, flat diamond radiator with as little mosaic spread as possible. The diamond radiator used by GlueX during the spring of 2017 to collect the data presented in this thesis was cut and polished from a $257.5 \mu \mathrm{m}$ crystal to a mean thickness of $58 \mu \mathrm{m}$ in the beam spot region. The surface of the crystal was imaged to test for consistent thickness (see Figure $2.2(\mathrm{a})$ ), and the mosaic spread of the crystal was tested with a 'rocking

\footnotetext{
${ }^{4}$ Reciprocal lattice vectors are momentum-space counterparts (Fourier transforms) of regular lattice vectors, which are position translation vectors between a reference lattice point (nucleus) and any other lattice point within a unit cell of a crystal.

${ }^{5}$ In the SM, photons have helicity of \pm 1 . These two helicities represent photons that have leftor right-handed circular polarization. Thus, a 'linearly polarized' photon is a coherent state of two superimposed photons with opposite helicity and appropriate phase difference and amplitude.
} 
curve.' A rocking curve is performed with the use of a monochromatic X-ray beam. The diamond is placed in the beamline with a photodetector placed at the angle of perfect Bragg reflection. Bragg's Law for a crystal with lattice spacing $d$ and an X-ray wavelength of $\lambda$ describes the angle at which the incident X-ray must be with respect to the crystal's surface for constructive interference of scattered photons, as shown in Figure 2.3.

a)

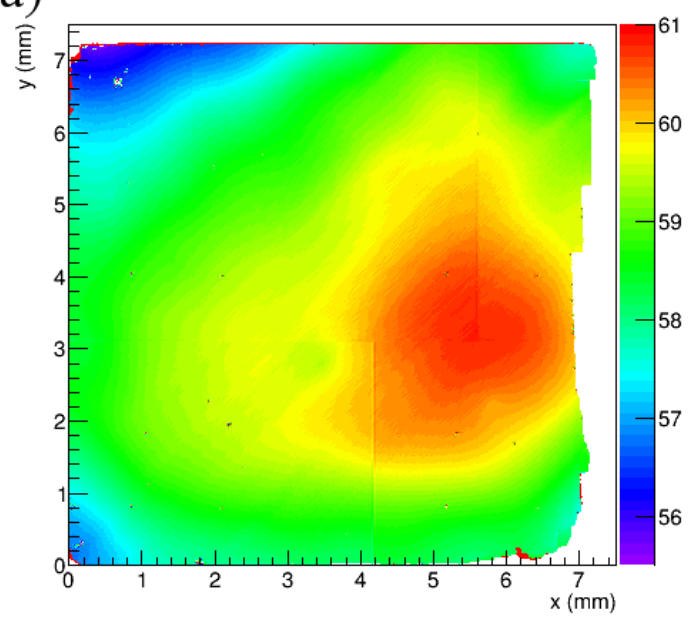

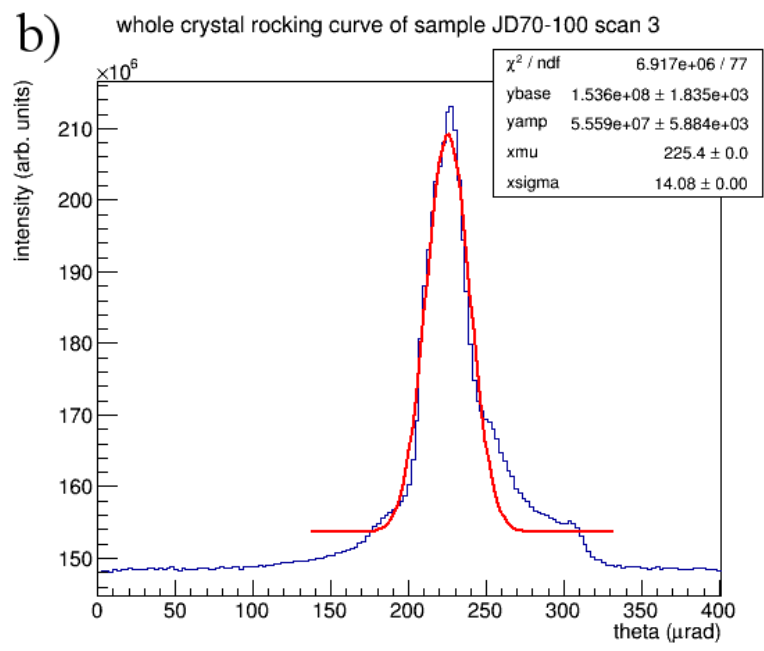

Figure 2.2: Thickness scan (a) and rocking curve (b) from GlueX diamond JD70-100, the radiator used during data taking in the spring of 2017. Images from Reference [88].

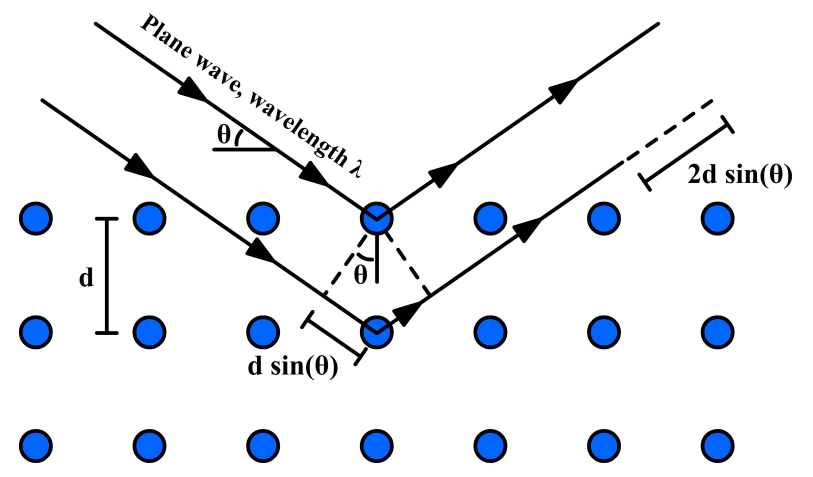

Constructive interference when path length difference is an integer number of wavelengths

$$
\begin{aligned}
& \text { 2d } \sin (\theta)=\mathbf{n} \lambda \\
& \text { 'Bragg's Law' }
\end{aligned}
$$

Figure 2.3: Bragg reflection of a plane wave on a crystal lattice. An incident plane wave generates a coherent reflected plane wave when Bragg's Law (described in the Figure) is satisfied. 
By tilting (rocking) the diamond over the range of Bragg reflection and measuring the intensity of the scattered photon beam in the photodetector, a rocking curve can be measured. This curve features a sharp peak at the diamond tilt that satisfies the Bragg condition. The width of the peak is an indicator of the uniformity of the diamond lattice. Non-uniformities cause reflections at varying angles, and so the mosaic spread of the crystal is measured as the width of the rocking curve. Several tests of the GlueX diamond show a rocking curve width of 20 to $50 \mu$ r (see Figure 2.2 (b) for one such test). Initial diamond scans were carried out at the CHESS facility ${ }^{6}$ When that facility switched its focus to protein scans, the GlueX tests migrated to the Canadian Light Source ${ }^{7}$.

Collimation of the photon beam helps clean up the beam to provide a high degree of polarization and a tighter energy spectrum by restricting the outgoing Bremsstrahlung angle. For the GlueX Experiment, the photon beam produced in the Tagger Hall is collimated through two collimators situated $75 \mathrm{~m}$ downstream of the radiator ${ }^{8}$. The first defines the angles of photons that will reach the target. Secondary photons and charged particles can be produced in this collimator by the parts of the beam that strike it, so a sweeping magnet placed after this collimator pulls charged particles out of the beam line. The second collimator is slightly larger and catches the secondary photons created by interactions in the first collimator [89]. The end result is a photon beam with a coherent peak centered at around $9 \mathrm{GeV}$ and a partial linear polarization on the order of $40 \%$, with the polarization angle of the beam controllable by rotating the diamond wafer accordingly (see Figure 2.4). For the purposes of finding the beam asymmetry for mesons (see Section 3.2) and checking for systematic effects, data for four beam polarization angles are collected:

\footnotetext{
${ }^{6}$ https://lightsources.org/lightsources-of-the-world/americas/cornell-high-energy-synchrotronsource-chess/

${ }^{7}$ https://www.lightsource.ca

${ }^{8}$ The distance from the radiator to the collimator, along with the beam spot size, affect greatly the polarization percentage of the beam, as discussed in Reference [87].
} 
$0^{\circ}$ (defined as parallel to the lab floor), $45^{\circ}, 90^{\circ}$, and $135^{\circ}$.
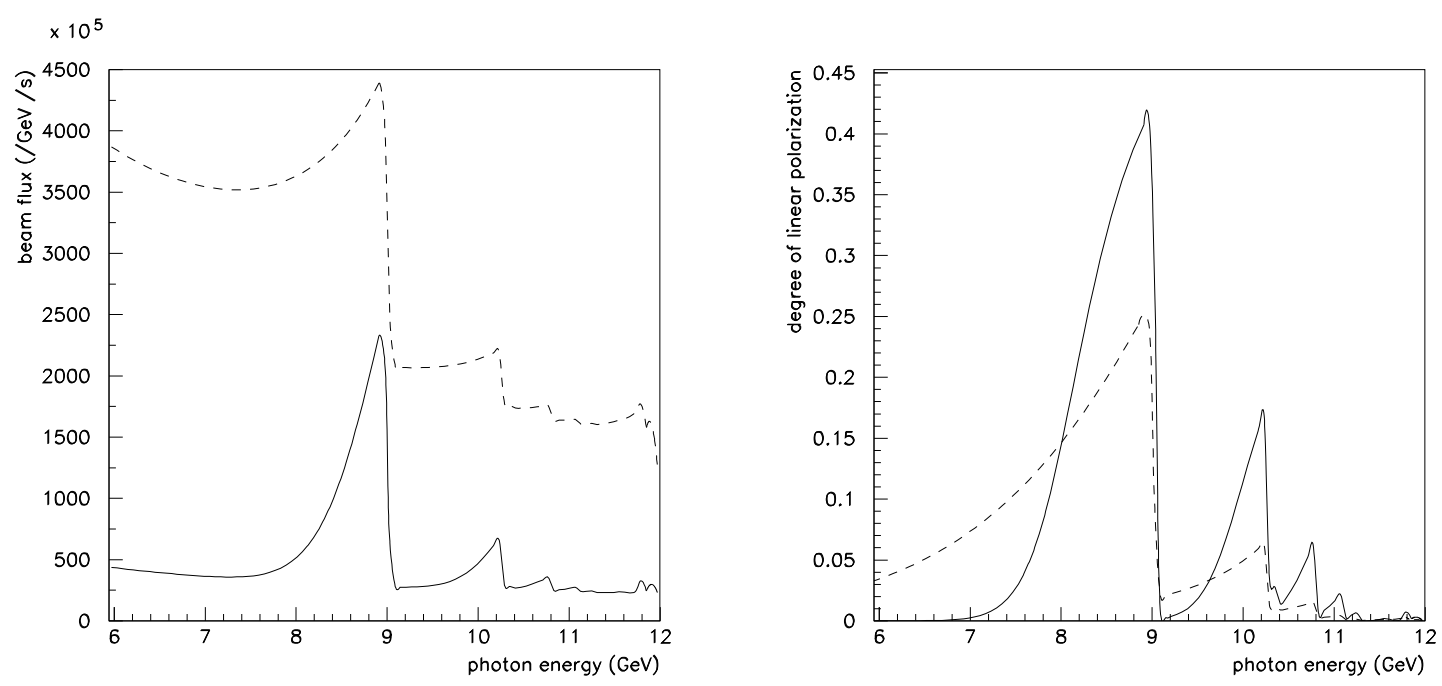

Figure 2.4: (Left) Theoretically calculated beam rate of the GlueX photon beam as a function of energy. (Right) Polarization of that photon beam as a function of energy. Simulated using a radiator thickness of $10^{-4}$ radiation lengths and a $3 \mu \mathrm{A}$ beam current. In both figures, the dashed line is the result with no beam collimation while the solid line includes a collimator $3.4 \mathrm{~mm}$ in diameter placed $80 \mathrm{~m}$ from the radiator. Figure from Reference [90].

\subsubsection{Tagger Hodoscope and Microscope}

Electrons that radiate Bremsstrahlung photons lose varying amounts of energy. A pair of dipole magnets placed just downstream of the radiator sweep the electrons out of the beam line towards a beam dump. The dipoles will disperse the electrons based on the energy they have. Electrons that radiated a 'harder' higher energy photon will bend more than an electron that radiated a 'softer' photon. The TAGH and TAGM are position-sensitive scintillation detectors placed near the dipole that measure the position of a bent electron, which is used to calculate the energy of that electron. This energy, along with the initial beam energy, can be used to determine the energy of the Bremsstrahlung photon. A photon that has a corresponding electron hit in one of the tagger detectors is referred to as a 'tagged' photon. 
The Tagger Microscope is placed to detect electrons with energies between 3.0 and 3.6 GeV, corresponding to Bremsstrahlung photon energies between 8.4 and 9.0 GeV, which is the energy range surrounding the coherent Bremsstrahlung peak. Each individual scintillator in the TAGM has a width corresponding to about $10 \mathrm{MeV}$ in photon energy. The Tagger Hodoscope scintillators cover higher and lower energies, down to $0.22 \mathrm{GeV}$ and up to $9.0 \mathrm{GeV}$ (3.0 to $11.8 \mathrm{GeV}$ photon energy), with wider scintillators of about $30 \mathrm{MeV}$ width in photon energy [89]. Figure 2.5 shows the dipole magnets and the tagger's focal plane, where the TAGH and TAGM scintillators lie.

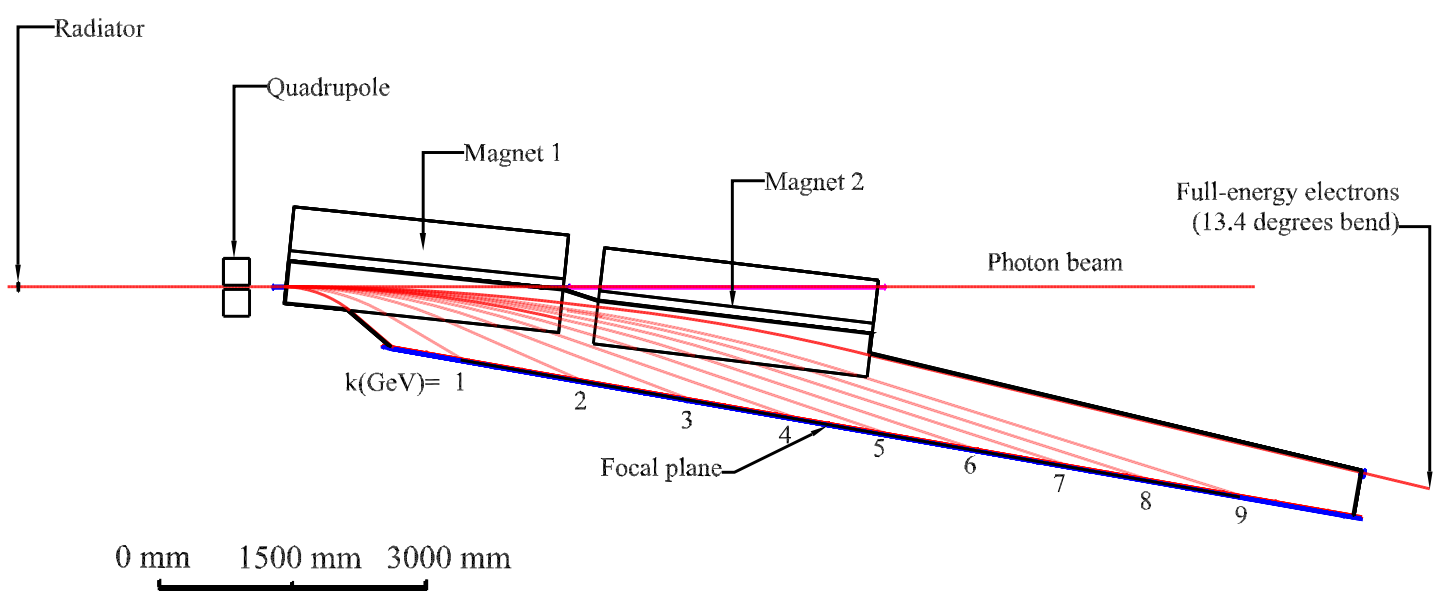

Figure 2.5: Rendering of the tagger dipoles and the focal plane of the TAGH and TAGM. Bent lines incident on the focal plan show electron trajectories for electrons of varying energies. Figure from Reference [89].

\subsubsection{Triplet Polarimeter}

After collimation, the beam's polarization is measured by the Triplet Polarimeter (TPOL). The polarized photon beam passes through a thin beryllium foil converter, which induces electron-positron pair production upon interaction of a photon with an atomic electron. The recoil (triplet) electron has a low energy, nearly independent of the incident photon energy, and its azimuthal recoil angle, $\phi$, is measured. The cross section for this process is given by 


$$
\sigma=\sigma_{0}(1+P \Sigma \cos (2 \phi))
$$

Here, $\sigma_{0}$ is the unpolarized cross section (known from previous experiments), $P$ is the photon beam's polarization, and $\Sigma$ is the beam asymmetry. This is a similar situation as measuring the beam asymmetry of a meson, discussed in Section 3.2. However, the recoil electron passing through the beryllium foil can also produce $\delta$ rays $^{9}$, which alter the angular spectrum. What is measured experimentally, rather than $\Sigma$, is called the 'analyzing power,' $\Sigma_{A}$. Since this process is entirely electromagnetic, the analyzing power for the process at various photon energies is predictable and can be found from simulation [91]. As such, a fit of the angular distribution of the recoil electron gives the beam's polarization. Then, the polarization can be measured for each of the four diamond orientations used and in bins of beam energy. The results of these measurements are shown in Figure 2.6 (with vertical error bars representing statistical errors), where it can be seen that the beam's polarization is highest in the coherent peak around $8.8 \mathrm{GeV}$, averaging around $35 \%$ for all diamond orientations.
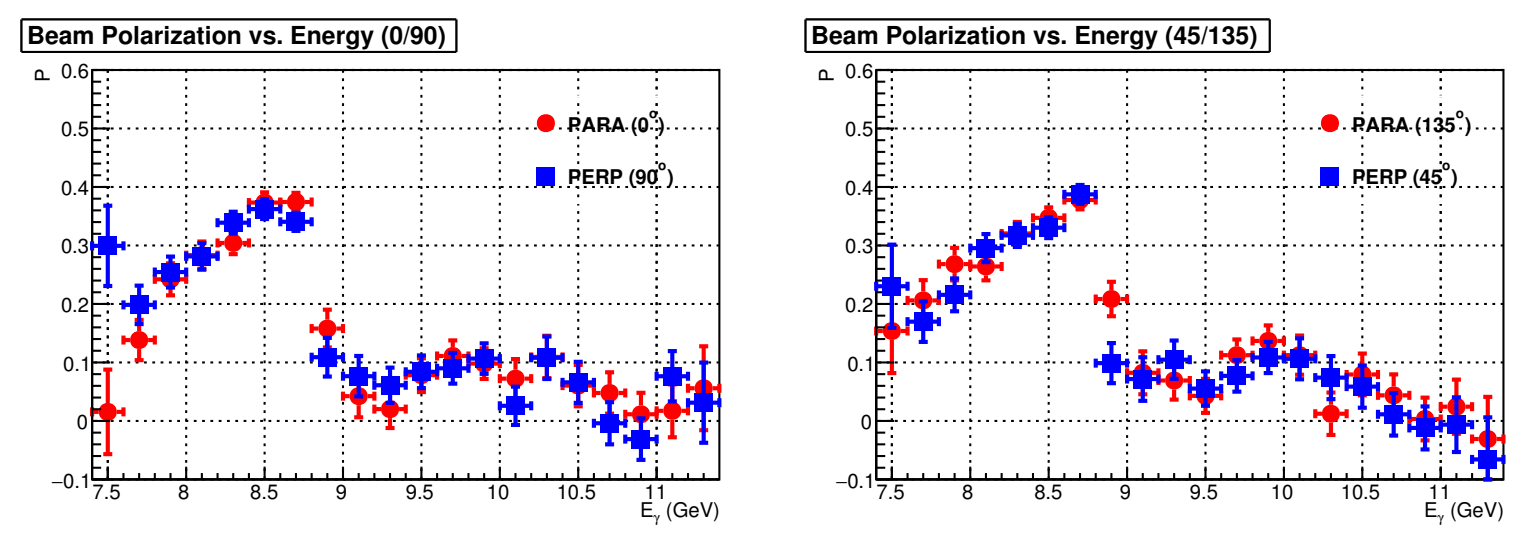

Figure 2.6: Polarization of the photon beam as measured by the TPOL as a function of energy for the four diamond orientation data sets. Statistical errors on each point are represented by vertical error bars.

\footnotetext{
${ }^{9}$ Secondary electrons created when charged particles traversing a medium kick orbital electrons out of atoms.
} 


\subsubsection{Pair Spectrometer}

The electron-positron pair produced in the beryllium converter before the TPOL proceeds to the Pair Spectrometer (PS). The electron and positron are diverted by a dipole magnet into a pair of hodoscopes, one on either side of the beamline, as in Figure 2.7. Course counting hodoscopes behind the high-resolution ones provide a coincidence trigger used to eliminate background and noise.

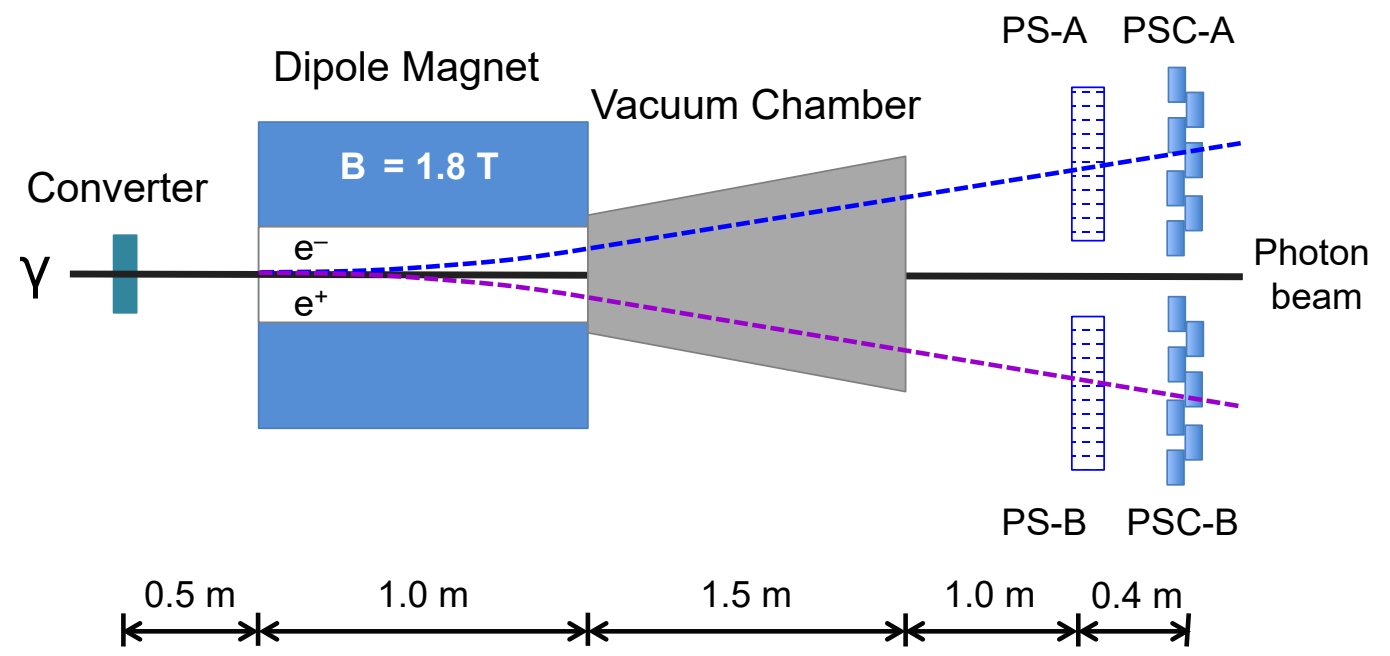

Figure 2.7: Schematic of the Pair Spectrometer. The electron-positron pair is separated by a dipole and the momenta of both particles is measured with a pair of hodoscopes. Figure from Reference [92].

Like the TAGH and TAGM, the electrons are deflected based on their energies, and so the energies of both the electron and positron can be measured. Each arm is sensitive to a momentum range of $3.0 \mathrm{GeV} / c$ to $6.2 \mathrm{GeV} / c$ for the leptons, which corresponds to photon energies between 6 and $12.4 \mathrm{GeV}$. This allows a measurement of the energy spectrum of the beam in and around the coherent peak region postcollimation and provides photon flux measurements for different diamond radiator orientations, the latter of which is shown in Figure 2.8 [92]. Measurements from the PS are also used to calibrate the TAGH and TAGM, and coincidence between a PS hit and TAGH/TAGM hit specifies the electron beam bunch responsible for the event. 


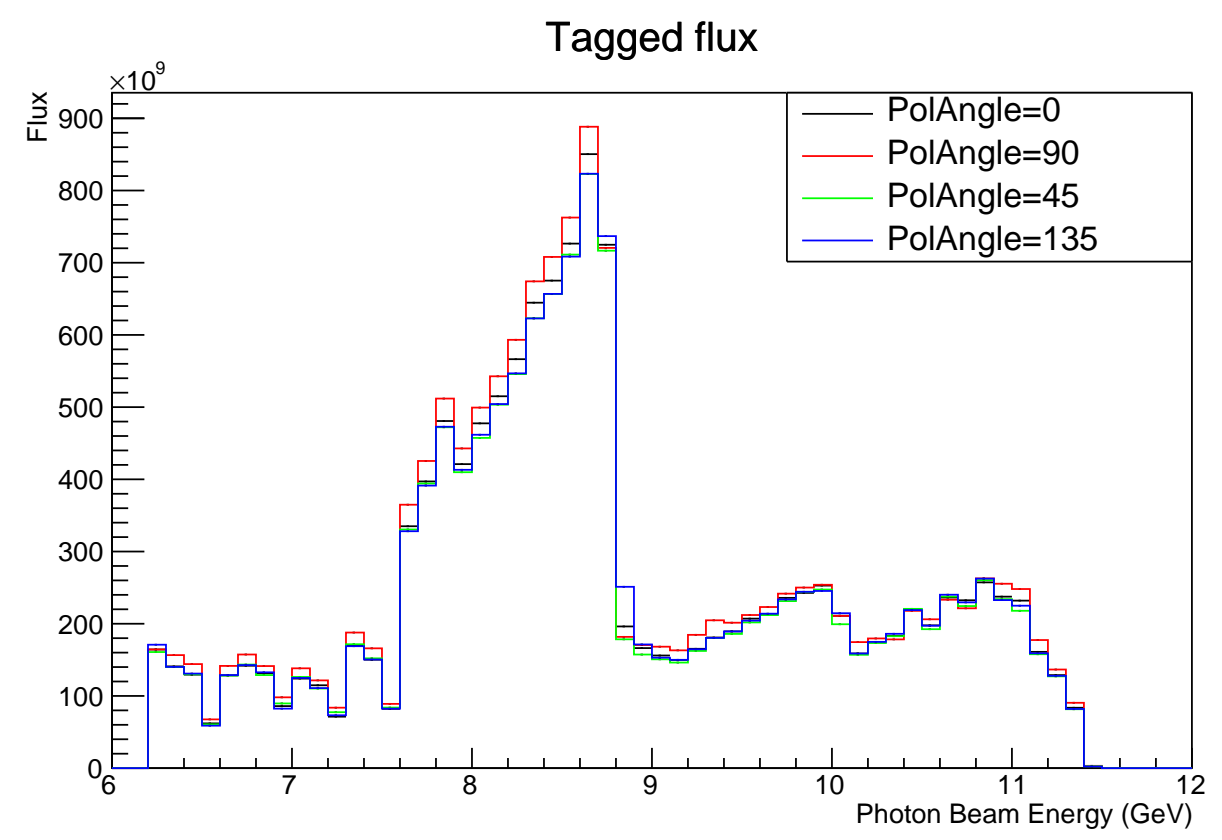

Figure 2.8: Photon beam energy distribution for PS events in coincidence with a TAGH or TAGM hit for the four photon polarization angles used.

\subsubsection{Liquid Hydrogen Target}

The spot size of the photon beam incident on the the GlueX nuclear target is a few $\mathrm{mm}$ in diameter [89] with a flux of around $10^{7}$ tagged photons per second. The target is a cryogenic liquid hydrogen (LH2) cell, $30 \mathrm{~cm}$ long with a $\sim 2 \mathrm{~cm}$ diameter (ensuring no beam scraping on the sides of the entrance beam pipe or target walls), held at a temperature of $\sim 20 \mathrm{~K}[93]$. Figure 2.9 shows a schematic of the GlueX target. The $30 \mathrm{~cm}$ long target provides $\sim 0.03 X_{0}$ (radiation lengths) of material for the photon beam.

\subsubsection{Charged Particle Tracking}

Surrounding the target is the nearly- $4 \pi$ hermetic detector consisting of two electromagnetic calorimeters, multiple charged tracking chambers, and scintillator arrays for timing measurements. Figure 2.10 depicts the detector and subsystems. 


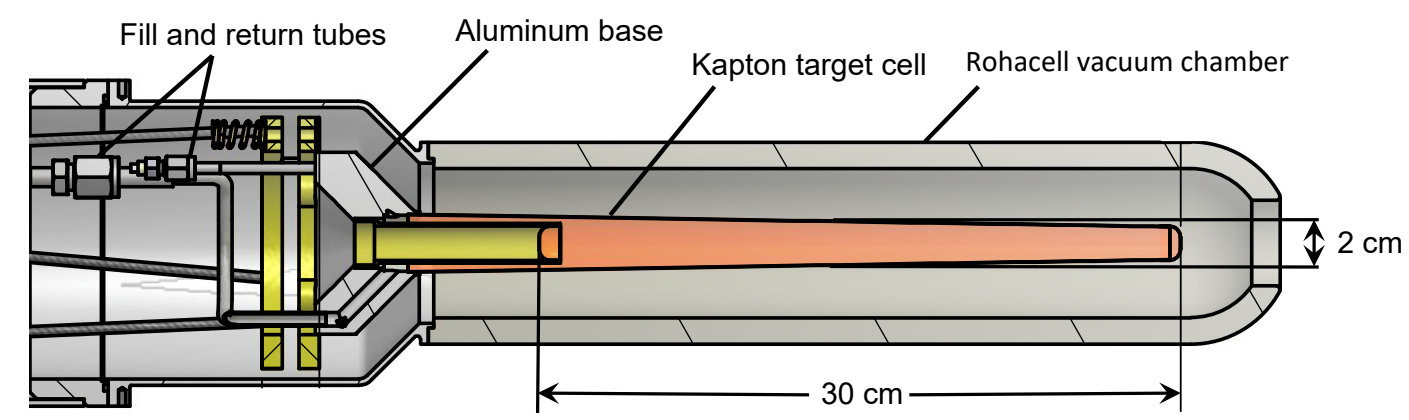

Figure 2.9: Schematic of the liquid hydrogen target used for the GlueX Experiment. The liquid hydrogen is contained in the orange-coloured cone. Figure from Reference [93] (colour online).

The GlueX detector sits inside of a large solenoid magnet capable of producing a 2.24 T magnetic field. Tracking of charged particles as they curve in the magnetic field, which is typically handled by drift chambers, provides momentum information about those charged particles. This information can be combined with other detected quantities to help determine the species of the charged particle. Furthermore, track and momentum information are useful for reconstructing the event vertex, the point from which the detected final state particles originated in the LH2 target.

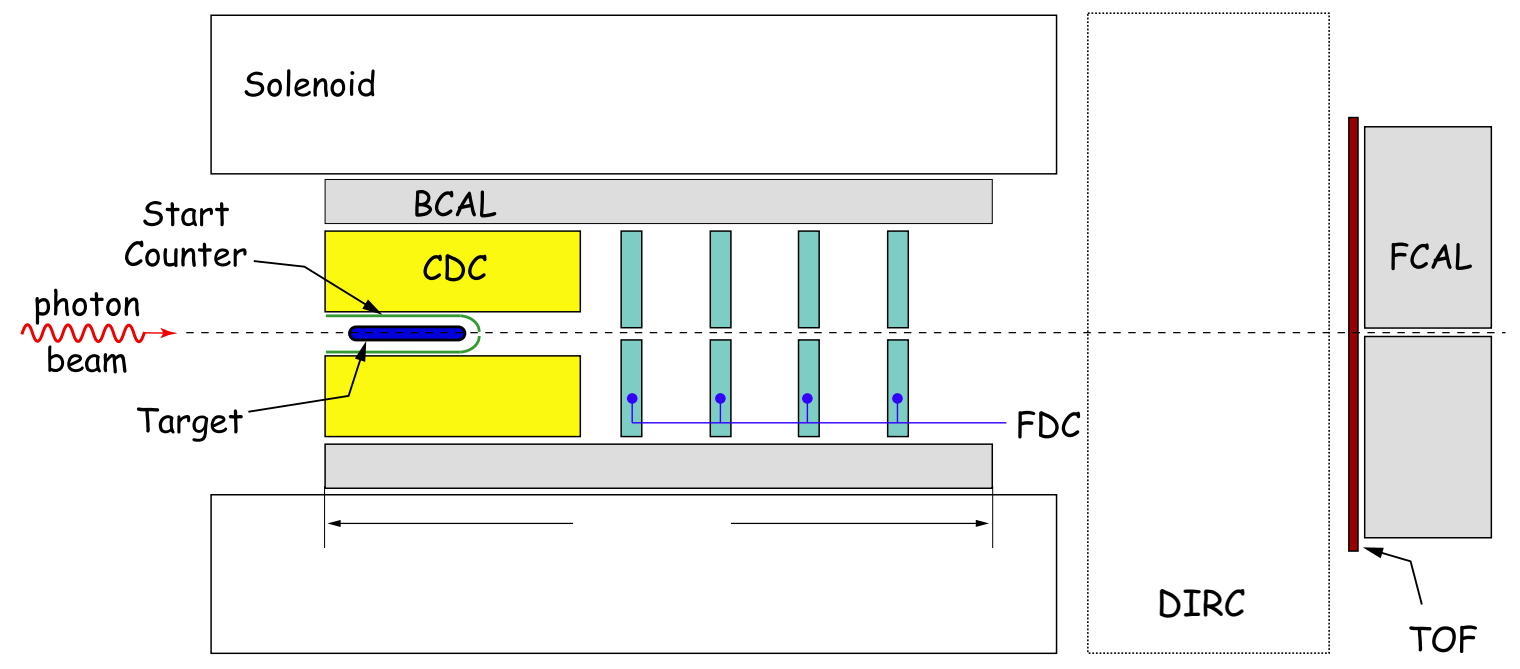

Detector is cylindrically symmetric about the beamline

Figure 2.10: Overview of detector subsystems in the GlueX detector. Figure from Reference [94]. 
Charged particle tracking for GlueX is handled by the Central Drift Chamber (CDC) and Forward Drift Chamber (FDC), discussed further in this section.

\subsubsection{Central Drift Chamber}

The $\mathrm{CDC}$ is a straw tube drift chamber that incorporates $3,5001.5 \mathrm{~m}$ long, $\sim 15.6 \mathrm{~mm}$ diameter straw tubes in a cylinder around the hydrogen target, covering a range in polar angle from the center of the target of $6^{\circ}$ to $165^{\circ}$. The straw tube walls are made of thin layers of kapton with one layer of aluminum on the inner wall, which serves as a cathode. In the center of a tube, a gold-plated tungsten wire with a diameter of $20 \mu \mathrm{m}$ acts as an anode. A mixture of argon and $\mathrm{CO}_{2}$ gases flows through the straws. When a charged particle passes through a straw, ionization of the gas occurs, with electrons drifting toward the cathode and ions drifting toward the anode. The drift time, which is the time between initial ionization and the first electron reaching the electrode, is measured with a high-resolution flash Analog-to-Digital Converter (fADC). The drift time is converted to a distance, and such information from many adjacent straw tubes allows reconstruction of charged particle tracks. In order to cut down on ambiguities in reconstruction, the straws are arranged in radial layers with three distinct orientations: axial (parallel with the beam line) and two stereo offsets ( $\pm 6^{\circ}$ from axial), as shown in Figure 2.11. The stereo straws help with measuring the position of a straw hit in $z$, along the beam direction. The CDC achieves a spatial resolution in $z$ of $2 \mathrm{~mm}$ and in the $\mathrm{r} \phi$ plane of $150 \mu \mathrm{m}$.

Another major contribution of this subsystem is particle identification (PID). To this end, the fADC is also used to estimate the amount of energy deposited in a straw. This energy loss, combined with the timing information which gives drift distance, allows a calculation of $\mathrm{dE} / \mathrm{dx}$, the amount of energy lost by the charged particle per unit distance in the gas. This quantity is helpful for distinguishing between protons and lighter charged particles at low momenta $[95,96]$. An example $\mathrm{dE} / \mathrm{dx}$ plot from 


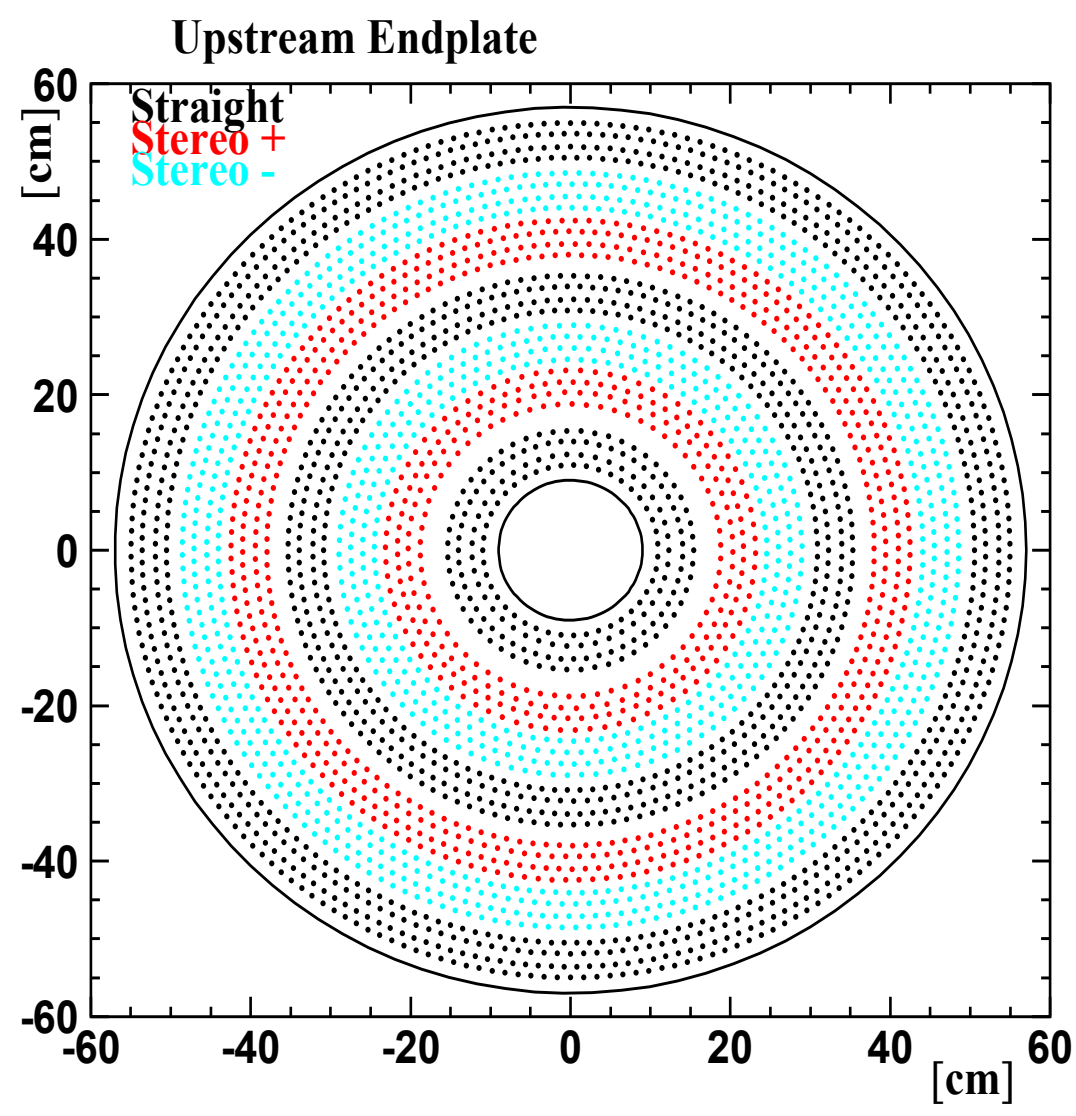

Figure 2.11: View from upstream of the CDC straw configuration. Depicted are the radial layers of axial straws (black dots) and stereo straws (red and blue dots, $\pm 6^{\circ}$ respectively). Figure from Reference [95] (colour online).

data is given in Figure 2.12. This is crucial for the identification of a recoil proton from the beam-target interaction, since usually such a proton has low momentum and will not be detected by other PID subsystems like the Time-of-Flight (see Section 2.2.4.2) or Barrel Calorimeter (see Section 2.2.3.1).

\subsubsection{Forward Drift Chamber}

The FDC is a set of four cathode strip detector packages placed downstream of the CDC. They detect forward-going charged particles in polar angles between $1^{\circ}$ and $20^{\circ}$. This style of drift chamber uses a plane of spaced anode wires and one or more planes of cathode strips at some angle with respect to the wires. Ionization of a gas 


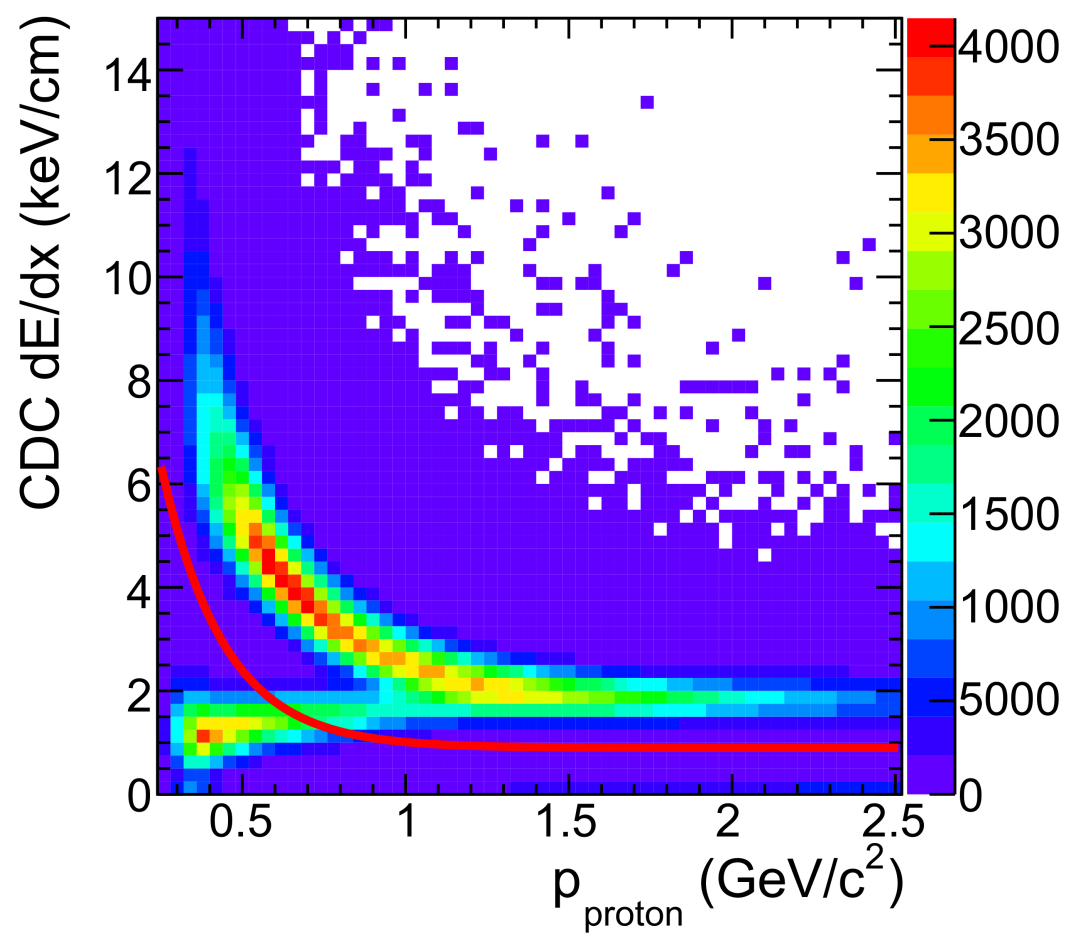

Figure 2.12: CDC dE/dx versus charged track momentum. The horizontal band below momenta of $1 \mathrm{GeV}$ is a result of lighter charged particles like electrons, pions, and kaons. The curved band is a result of protons. Above $1 \mathrm{GeV}$ or so, the two bands become indistinguishable. The red curve shows an extremely loose cut meant to exclude obvious light particles while preserving all proton candidates at low momentum in the early stages of an analysis (colour online).

(a mixture of argon and $\mathrm{CO}_{2}$ for the GlueX FDC) causes a current in the same way as other drift chambers. Data readout from the wires gives positional information in that direction while data read out from the strips gives the same in another direction, allowing a localization of the charged particle's passage through the face of the planes.

The GlueX FDC uses two cathode strip planes, rotated $\pm 75^{\circ}$ from the wire direction (depicted in Figure 2.13 (a)). One cathode plane sits downstream of the wire plane and one sits upstream ('U strips' and 'V strips' in the figure, respectively). The use of multiple planes at varying angles allows hit localization in the event of multiple simultaneous hits, not an uncommon occurrence at forward angles for highenergy production interactions. Furthermore, each of the four FDC packages contains 
six sets of wires and planes, with each set in a package rotated $60^{\circ}$ from its neighbours. A side view of an FDC package is shown in Figure 2.13 (b). The many layers of cathode strip detectors allows for spatial resolution on the order of $200 \mu \mathrm{m}$ [97], helpful for matching tracks from the CDC to hits in the two downstream detectors, the Forward Calorimeter and the Time-of-Flight.
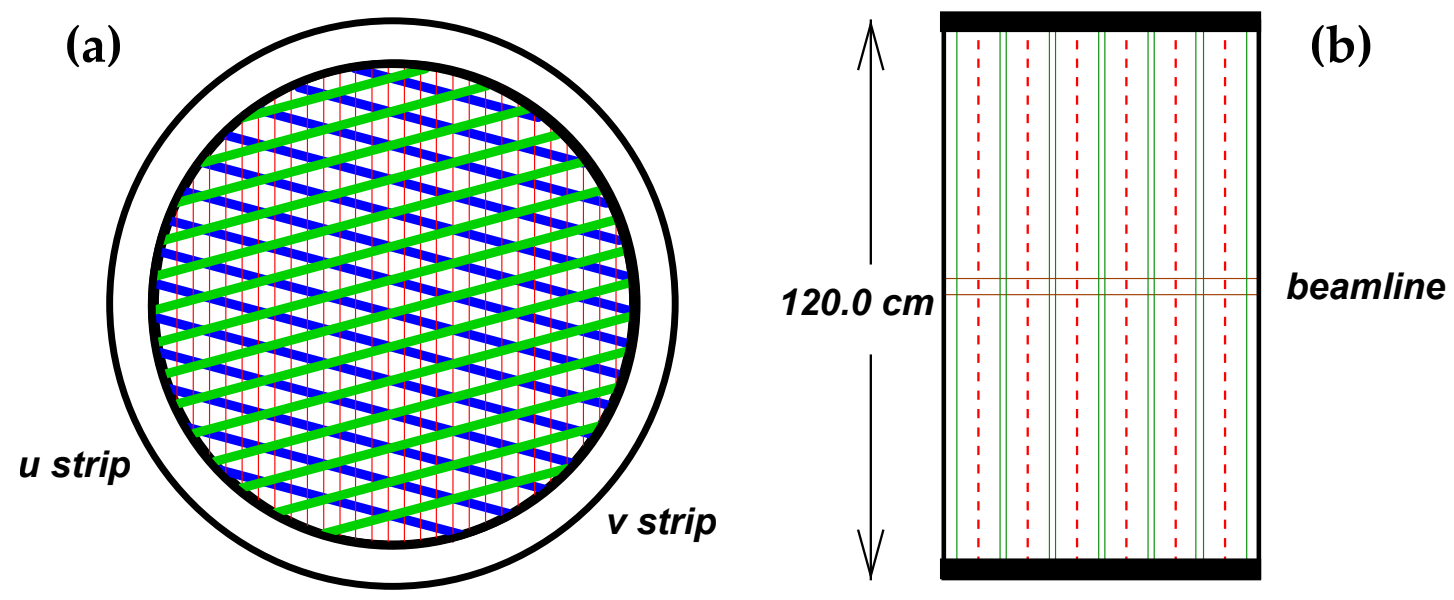

Figure 2.13: (a) Front view of an FDC package. Wires are shown as vertical lines. Two cathode strip planes lie at $\pm 75^{\circ}$ with respect to the wires, one on either side of the wires (U and V strips). (b) Side view of an FDC package. Wire planes are shown as dashed lines, while cathode planes are shown as solid lines. A package contains six sets of cathode strip detectors, each rotated $60^{\circ}$ from the previous one. Figure from Reference [97].

\subsubsection{Calorimetry}

Calorimetry is done with two electromagnetic calorimeters, the Barrel Calorimeter (BCAL) and the Forward Calorimeter (FCAL), discussed in this section. The former is a cylinder that surrounds the CDC and FDC, and the latter is a plane downstream of the solenoid. The geometry is such that any neutral particle produced near the hydrogen target at any angle other than steeply backwards can be detected by one of these calorimeters. During data analysis, positional information from calorimeter hits can be compared to charged track information to match tracks to calorimeter 
hits. Hits that are not matched are then likely from neutral particles (photons or neutrons).

\subsubsection{Barrel Calorimeter}

The BCAL is a cylindrical electromagnetic calorimeter, which sits radially between the solenoid and the drift chambers. The purpose of this calorimeter is to detect the energy and position of charged and neutral particles between polar angles of $11^{\circ}$ and $126^{\circ}$. The subsystem is composed of 48 trapezoidal modules $3.9 \mathrm{~m}$ long and $25 \mathrm{~cm}$ tall (15.3 radiation lengths, and up to 67 radiation lengths for particles incident at $14^{\circ}$ polar angle). Each module is composed of lead (to induce electromagnetic or hadronic showers) and scintillating fibers (to convert a shower's deposited energy into measurable photons). Stacked radially outward are 185 layers of alternating $0.5 \mathrm{~mm}$ thick lead sheets with grooves along their lengths and double-clad $1 \mathrm{~mm}$ diameter scintillating fibers ${ }^{10}$ glued into those grooves [98]. Particle showers, induced in or before the BCAL, deposit energy in the scintillating fibers that re-emit light, which travels down the fibers to both the upstream and downstream end of the BCAL to be measured by photodetectors. The amount of light produced by the scintillating fibers is directly proportional to the energy deposited in the fibers, and the amount of energy deposited in the entire calorimeter is predictable based on the amount of energy deposited in the fibers by comparison with simulations ${ }^{11}$. The upstream and downstream ends of each BCAL module are covered with 40 light guides, for a total of 3840 SiPMs. Each of these capture light from a $\sim 2 \mathrm{~cm}^{2}$ area worth of fibers and delivers it to a silicon photomultiplier (SiPM) ${ }^{12}$. A SiPM's 'dark count rate,' which is

\footnotetext{
${ }^{10}$ Kuraray Plastic Scintillating Fibers (http://www.kuraray.com/products/plastic/psf.html).

${ }^{11} \mathrm{~A}$ scaling factor between the energy deposited in the fibers and the total energy deposited in the calorimeter can be found through simulation of single-photon events. This scaling factor is refined through calibration of the subsystem, which for the BCAL involves matching the $\pi^{0}$ peak in a diphoton invariant mass spectrum from data to the true value of the $\pi^{0}$ mass.

${ }^{12}$ Standard photomultiplier tubes (PMTs) are not usable inside the intense magnetic field of the solenoid. SiPMs offer a light collection system that is effectively immune to magnetic fields.
} 
the rate of false photon signals in the absence of incident light, decreases dramatically with lower operating temperatures [99], so the SiPMs are placed in thermal contact with metal plates that are held at a low temperature (on the order of $7^{\circ} \mathrm{C}$ ) with a liquid cooling system. In order to prevent condensation on the faces of the SiPMs, which would interfere with the detection of light from the light guides, nitrogen gas is flushed through the upstream and downstream ends of the BCAL. The 40 SiPMs from a BCAL module are summed into four 'layers' (radial regions) and four 'sectors' (azimuthal regions), giving 16 readout channels ('cells') per BCAL module per side (see Figure 2.14).

Each of these readouts provides energy and timing information for energy depositions in those cells. By grouping nearby (in time and space) depositions, measurements of an electromagnetic or hadronic shower's energy and position as it entered the BCAL can be found. Primarily, this information is useful for reconstructing invariant masses of decaying neutral particles. For example, if two neutral showers are detected in the BCAL, the energy and position of the two showers measured by the calorimeter can be used to reconstruct their four-momenta (either through measurement of the vertex position using charged tracks, or by an assumption about the vertex position). Adding the four-momenta together yields the four-momentum of a particle that might have decayed to produce the two photons. By calculating the invariant mass of this new four-momentum and comparing to well-known mesons that decay to two photons, one can test if the two photons are likely to have come from a well-known particle (see Chapter 3 for more detailed discussion).

In addition to position and energy information, the BCAL can also be used for particle identification. For charged particles with large enough momenta ${ }^{13}$ (and the correct polar angle) to reach the BCAL, the flight times of the particles (accessed by the time difference between the BCAL hit and a hit in the Start Counter, for instance)

\footnotetext{
${ }^{13}$ Since the flight time between the target and the BCAL is short, there is an upper limit on the momentum of a charged particle based on the timing resolution of the subsystem.
} 


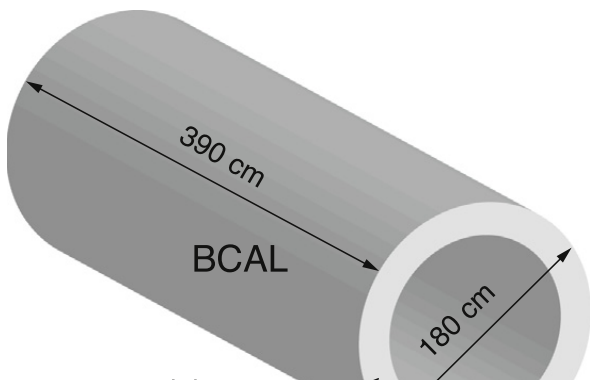

(a)

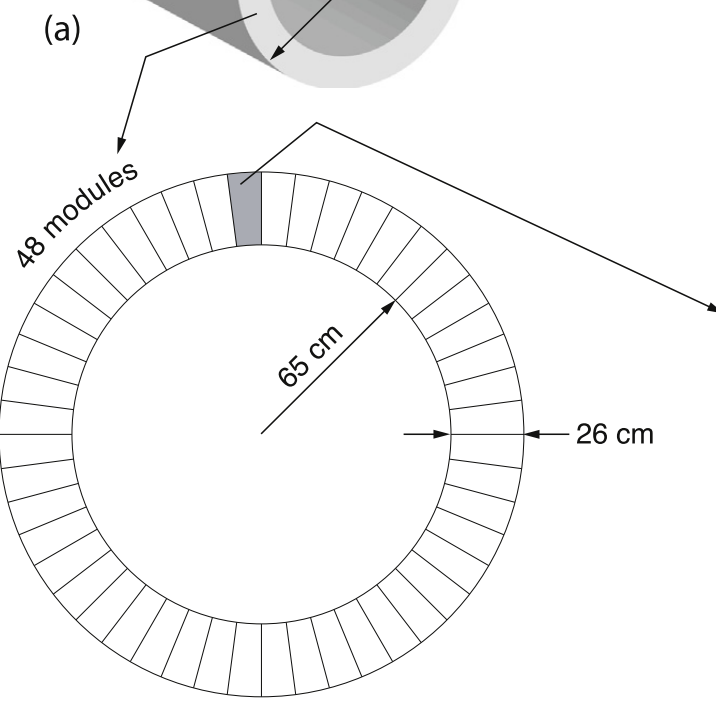

BCAL end view

(c)

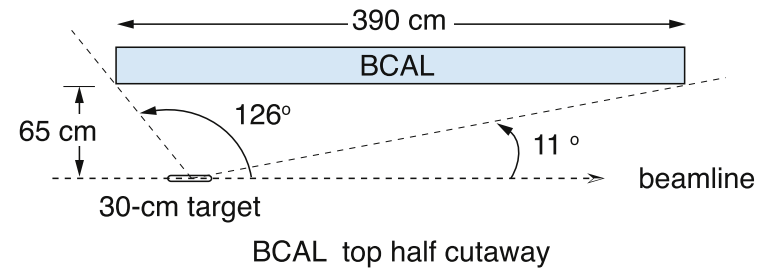

(b)

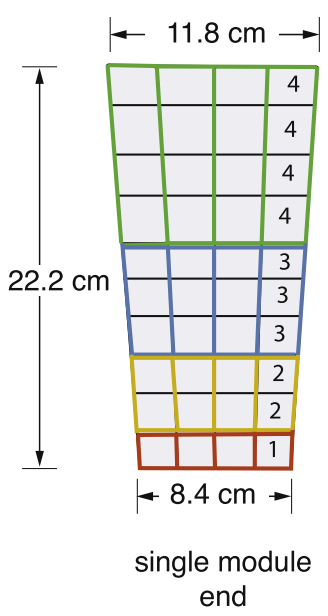

(d)

Figure 2.14: (a) Macro dimensions of the BCAL. (b) cross sectional (top-half only) view of the BCAL showing it's position with respect to the hydrogen target. (c) End view of the BCAL showing the 48 trapezoidal modules together forming the cylinder. (d) End view of one BCAL module showing the 40 light guide footprints and the layer-wise summing scheme used to produce the final 16 readout channels. Figure from Reference [98].

can be used to distinguish between different species of particle. Figure 2.15 shows the speed of charged particles calculated from BCAL showers that were matched to charged tracks versus the momentum of those particles. Two distinct bands are seen below a particle momentum of $2 \mathrm{GeV}$ showing clear separation between electrons/pions $(\beta \approx 1)$ and protons (lower speed band).

The SiPMs are monitored by periodically flashing LEDs (Light Emitting Diodes) in the light guides. The timing and energy data collected from these LED pulses are used to ensure the photodetectors have approximately correct timing and energy 


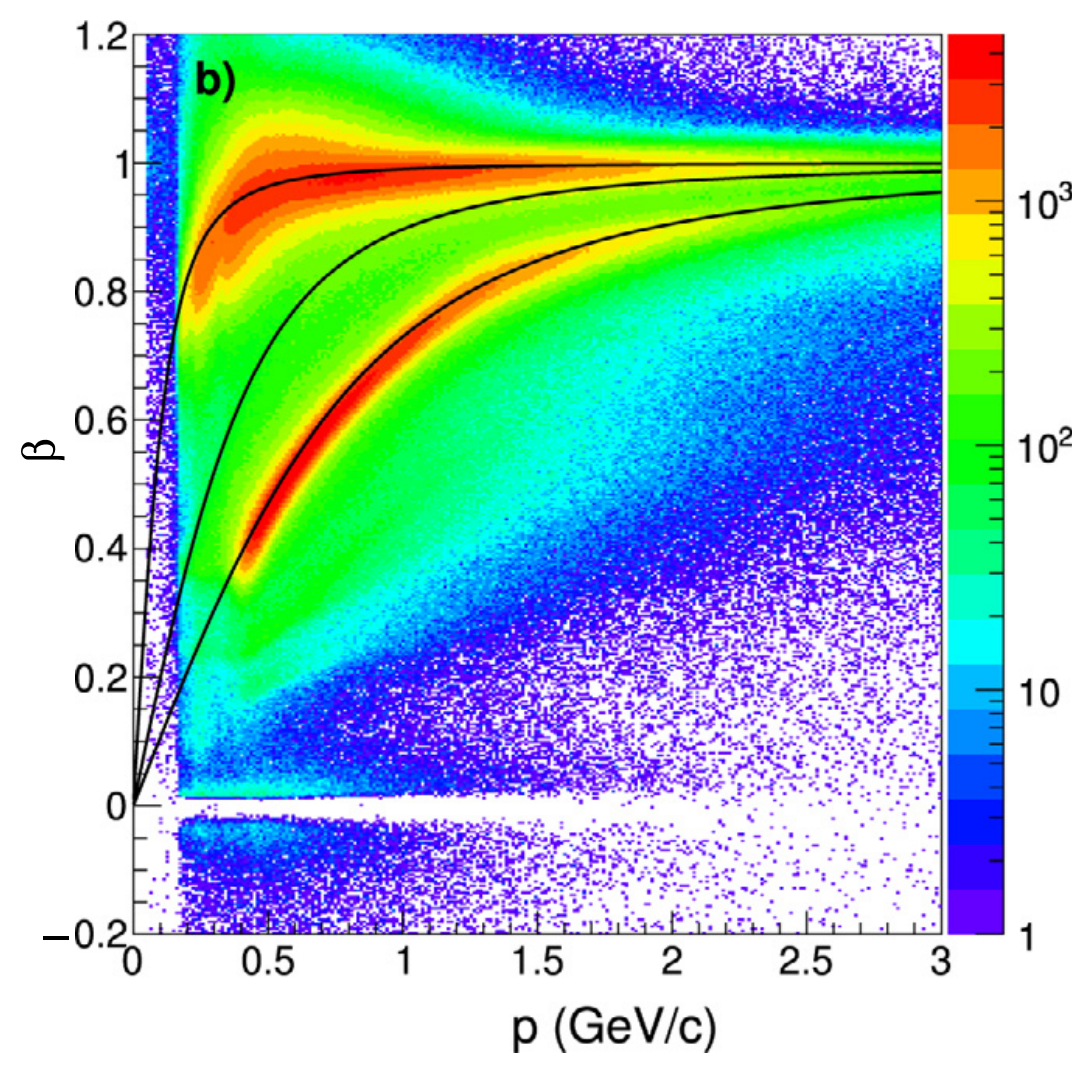

Figure 2.15: Measured speed ( $\beta$, the particle speed divided by the speed of light) of positively charged particles found using BCAL shower time information versus particle momentum. The three curves, from top to bottom, correspond to the expected distributions of pions, kaons, and protons.

characteristics. The latter is used for relative monitoring of gains. A similar LED system is used for gain monitoring in the FCAL [100].

\subsubsection{Forward Calorimeter}

The FCAL lies downstream of the rest of the detector and fulfills the same role as the BCAL, but for polar angles below $12^{\circ}$. This subsystem is constructed by stacking $28004 \times 4 \times 45 \mathrm{~cm}^{3}$ lead-glass blocks into a roughly circular shape of about $1 \mathrm{~m}$ radius [101]. A square of 9 blocks is omitted at the center of the FCAL, termed the 'beam hole,' so the photon beam can pass through to the beam dump at the end of the hall (see Figure 2.16). 


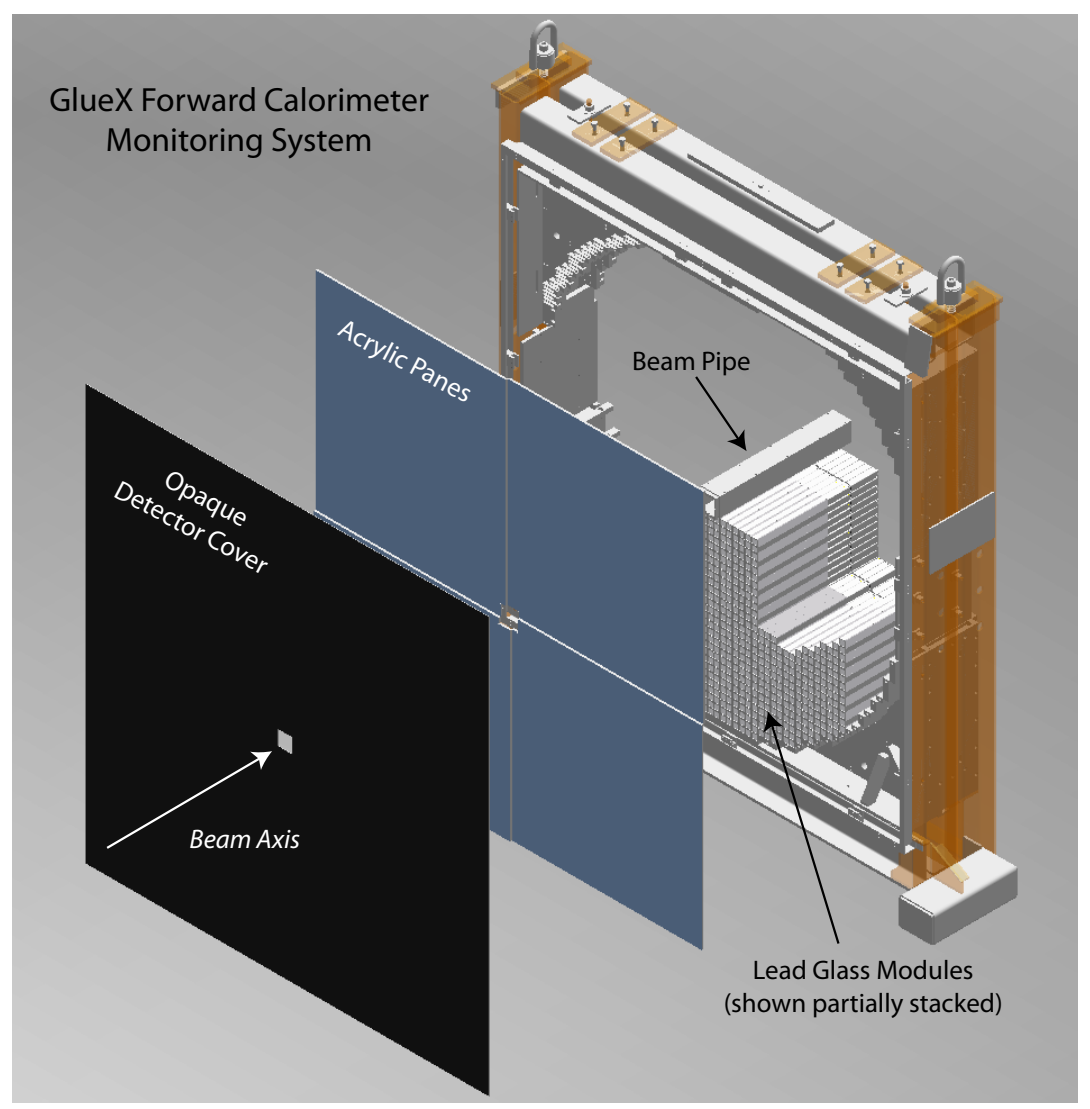

Figure 2.16: Rendering of the GlueX FCAL, showing the partially-stacked lead-glass blocks, the beam hole, and the acrylic planes used for gain monitoring ${ }^{14}$. Figure from Reference [100].

Particles traversing a lead-glass block cause particle showers, and the high-speed charged particles from those showers produce Cherenkov light ${ }^{15}$ that is read out by photomultiplier tubes at the back of each lead-glass block. Since the amount of Cherenkov light produced is again dependent on the amount of energy deposited by the initial particle, the total energy of the shower can be found by grouping together signals from adjacent lead-glass blocks. As with the BCAL, hits can be matched to charged tracks to differentiate charged and neutral showers in the FCAL.

The placement of the FCAL, $5 \mathrm{~m}$ downstream of the hydrogen target, is to help

\footnotetext{
${ }^{14}$ Light from LEDs located on the edges of these acrylic planes illuminates the lead-glass blocks for monitoring the photodetectors' timing and energy characteristics. The opaque covering on the outside of the acrylic planes protects the acrylic from ambient light.

${ }^{15}$ Cherenkov light is produced by a charged particle travelling through a dielectric (electrically polarizable) medium faster than the speed of light in that medium.
} 
with resolving power [89]. Multiple high-energy photons produced from the decay of a meson may travel close together at very forward angles, and having the photons travel a longer distance increases the chance that they will strike the FCAL in two distinct places that can be cleanly separated for energy and timing measurements rather than striking the same or adjacent lead-glass blocks, making it impossible to tell how many photons exist in the final state.

The FCAL can also be used to identify electron hits from hadronic ones. Figure 2.17 shows an example data plot of the ratio of FCAL shower energy to matched charged track momentum versus the track's polar angle. Charged particles with an energy to momentum ratio of around 1 correspond to low mass electrons and positrons, which are distinctly separable from the horizontal band of hadrons.

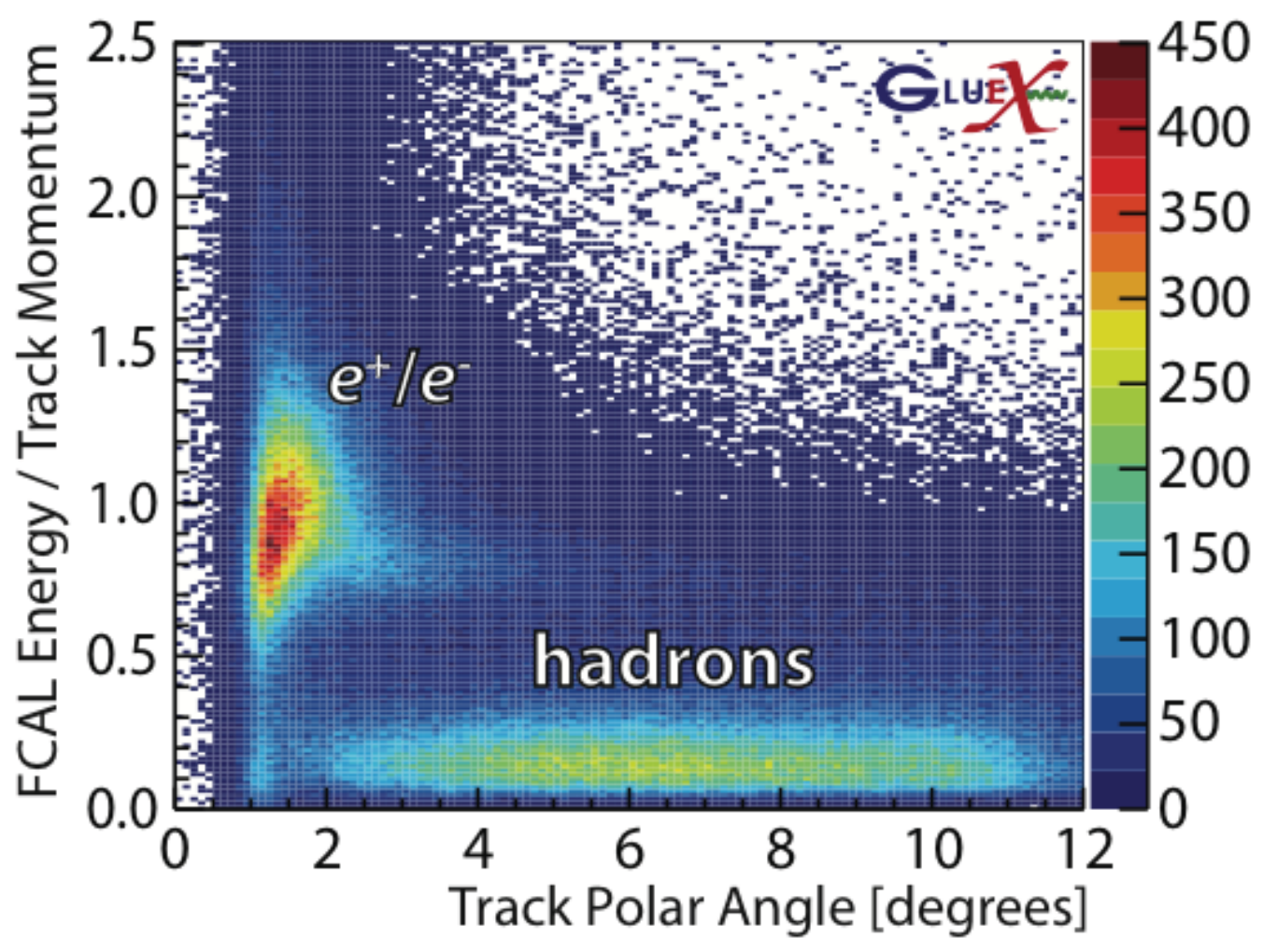

Figure 2.17: Ratio of energy, as measured by the FCAL, to charged particle track momentum versus track polar angle. Using energy information from the FCAL, identification of hits as being from electrons or positrons as opposed to hadrons is possible. Figure from Reference [102]. 


\subsubsection{Particle Identification}

Particle identification (PID) is carried out in a variety of ways using most of the detector subsystems. Energy deposition information from the CDC and calorimeters can differentiate between light and heavy charged particles at low momentum, for instance, but for high-momentum, forward-angle particles, the Time-of-Flight (TOF) provides timing information for distinguishing particle species. The Start Counter (ST), located just outside the hydrogen target, is a crucial subsystem for measuring flight times to any detector. These two subsystems are discussed in this section. The recent PID upgrade to the GlueX detector, the DIRC (Detection of Internally Reflected Cherenkov light) detector, is also discussed.

\subsubsection{Start Counter}

The Start Counter is an array of 12 scintillator bars surrounding the hydrogen target and covering polar angles between $7.5^{\circ}$ and $149^{\circ}$ (see Figure 2.18). Each bar is bent by applying localized infrared heating and forming the bend around an aluminum drum to match the shape of the target chamber. Particles produced in the target pass through the bars, producing scintillation light that is detected by SiPMs.

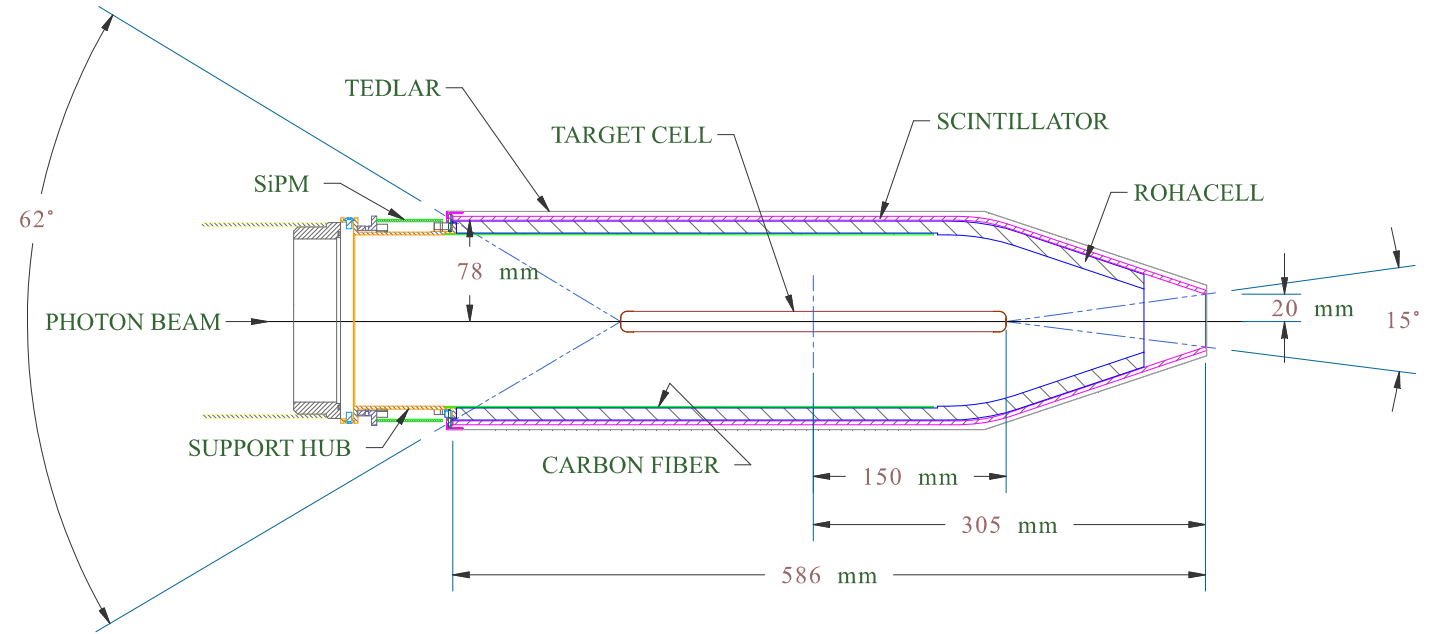

Figure 2.18: Schematic view of the Start Counter. Figure from Reference [103]. 
The main purpose of the ST is to identify the RF beam bunch that corresponds to a production event. Timing information from the SiPMs is used to measure the time of an interaction between a beam photon and the target, which can then be compared to the RF beam bunch timing. Because the beam bunches arrive at $\sim 4 \mathrm{~ns}$ intervals (249.5 MHz frequency), the timing resolution of this subsystem must be on the order of $1 \mathrm{~ns}$ to resolve the correct RF beam bunch responsible for the interaction. The design reolution of the ST is a few hundred ps, which satisfies the requirements for resolving RF beam bunches and also facilitates PID with other detectors through comparison of hit times in those subsystems with the well-known ST time. The ST itself can also be used for PID by measuring the energies of hits along with the times. For hits matched to charged tracks, the energy loss $(\mathrm{dE} / \mathrm{dx})$ can be plotted versus the momentum of the particle in much the same way as for the CDC. Two bands, one for the proton and one for the lighter charged particles (electrons, pions, and kaons) are clearly distinguishable up to about $0.9 \mathrm{GeV} / c$ [103]. This can be useful for identifying low momentum recoil protons that do not make a distinguishable track in the CDC, for instance.

\subsubsection{Time-of-Flight}

For higher-momentum, forward-angle particles, use of the TOF is essential for separation of protons, kaons, and pions. Because of the long flight distance from the target to the TOF wall, distinguishing particles based on timing is possible up to higher momenta than other subsystems like the BCAL. In addition, the timing resolution of the TOF is also good, on the order of $100 \mathrm{ps}$. The subsystem features two layers of $252 \mathrm{~cm}$ long, $6 \mathrm{~cm}$ wide scintillator bar arrays aligned perpendicularly to one another. Each bar features two photomultiplier tubes to read out energy and timing information from hits. A $12 \times 12 \mathrm{~cm}^{2}$ square hole in the center matches the beam hole in the FCAL to allow traversal of the photon beam through to the beam 
dump. Here, half-length bars are used on either side of the beam hole attached to only one PMT. Additionally, two half-width bars flank either side of the beam hole in both layers [104] (see Figure 2.19). This helps with the high event rate expected just outside the beam hole and with distinguishing tightly-packed high momentum particles.

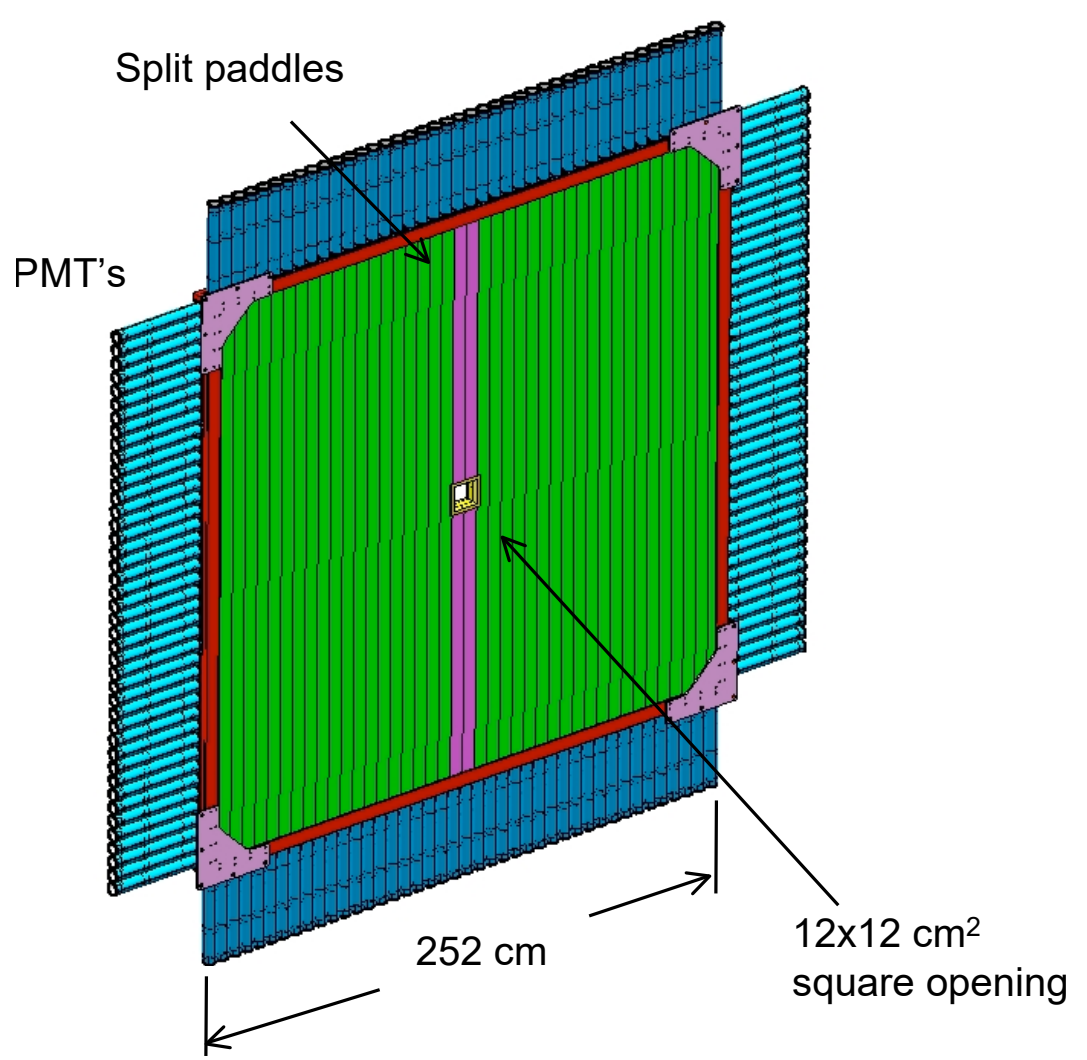

Figure 2.19: Early conceptual rendering of the TOF wall. Note that the half-width scintillator bars on either side of the beam hole are not represented in this rendering. Figure from Reference [105]

An example data plot of positively charged particles' speed as measured by the TOF versus their momenta is shown in Figure 2.20. Bands of data for positrons, pions, kaons, and protons can be seen and separated in various momentum ranges. Most prominently, the proton band can be separated from the rest of the lighter charged particles up to around $3 \mathrm{GeV} / c$. Pion-kaon separation is possible with the TOF, but only up to $2 \mathrm{GeV} / c$ or so where the kaon band merges with the pion and 
electron bands.

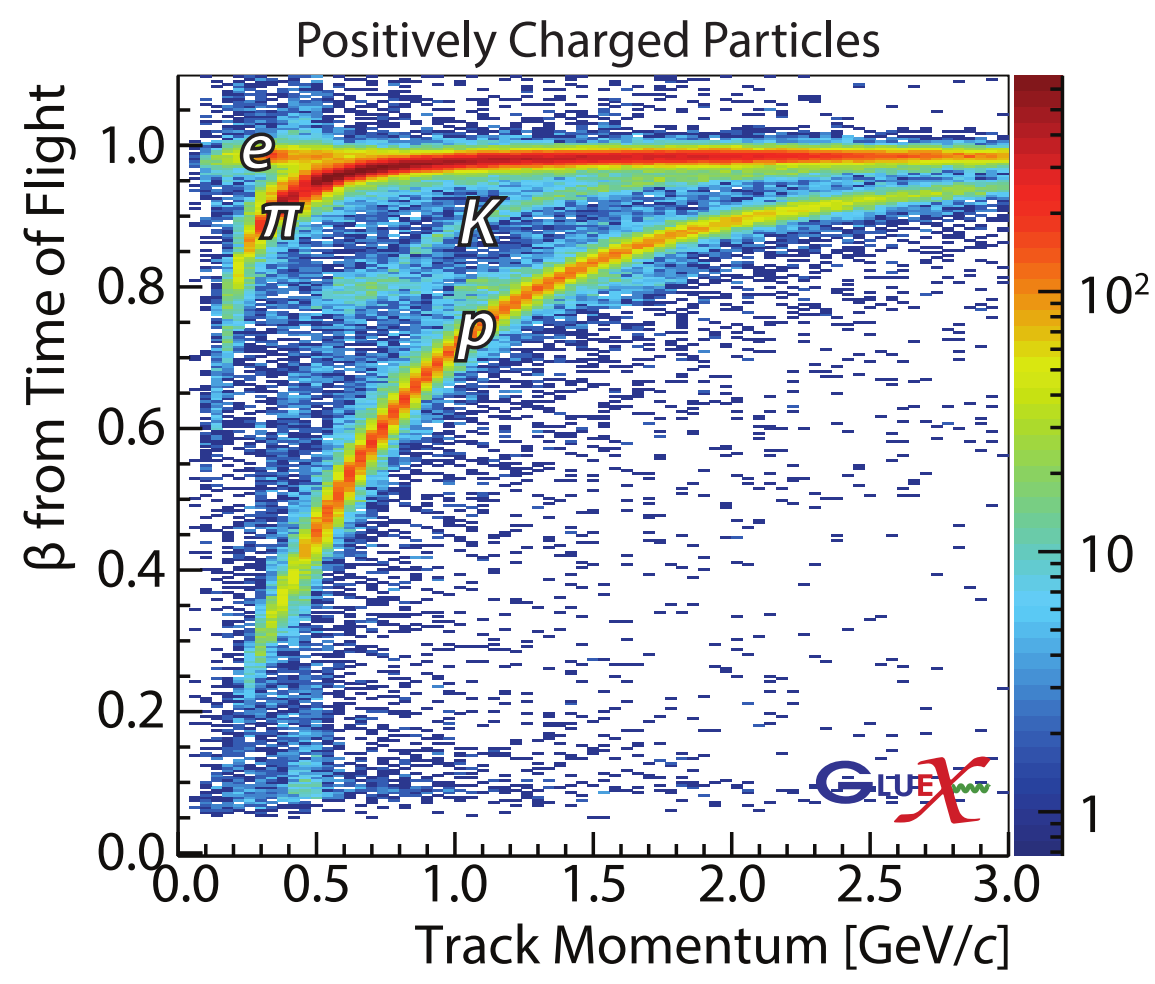

Figure 2.20: Speed, as measured by the TOF, of positively charged particles versus momentum showing separation of the positron, pion, kaon, and proton bands in various momentum ranges. The faint, horizontal band around $\beta=0.8$ is caused by 'accidentally-tagged' (see Figure 3.1 and associated text) positron and pion tracks. Figure from Reference [105].

\subsubsection{Recent Upgrade: DIRC}

Several hybrid meson decay modes involve charged kaons, and in order to fully study potential hybrids with strange quark content, kaon identification up to higher momentum is required. To this end, a DIRC detector has been installed in the gap between the TOF and the rest of the GlueX detector ${ }^{16}$. It is a Cherenkov detector that uses fused-silica bars from the BaBar Experiment's DIRC detector [106]. Four BaBar bar boxes are used for the GlueX DIRC, two above the beam hole and two

\footnotetext{
${ }^{16}$ Installation and commissioning of half of the DIRC took place in January and February, 2019. Installation of the remaining half of the detector is planned to take place after the current run period is complete (late April).
} 
below, each horizontally oriented, with the whole setup covering polar angles between $2^{\circ}$ and $11^{\circ}$.

Each bar box contains 12 fused-silica bars, each $17 \mathrm{~mm} \times 35 \mathrm{~mm} \times 4.9 \mathrm{~m}$ in size. As a charged particle passes through a bar, if the speed of the particle is larger than the speed of light in the bar $(c / n, n$ being the index of refraction of the bar), the particle will radiate Cherenkov light at a characteristic angle based on the speed of the particle. In a large-volume Cherenkov detector, the cone of light from this process might be picked up by an array of PMTs or other sensors, and the size of the ring would define the angle and thus the speed of the particle. Coupled with momentum information, this can uniquely identify the particle species. At BaBar or GlueX, this is not possible because of space limitations caused by the placement of other detector subsystems. So, in the fused-silica bars, the Cherenkov light is totally internally reflected to one end of the long bar (a mirror lies at the other end) where the light enters an expansion chamber. The light is directed by mirrors in this chamber onto a plane of photosensors, and since the Cherenkov angle of the emitted light is preserved through reflections, the pattern on the plane of photosensors can be analyzed to yield the same speed information as in a standard large-volume Cherenkov.

The expansion chamber (or 'optical box') used for GlueX is shown schematically in Figure 2.21 and simulations of the optical pattern of Cherenkov light from the photosensors in the expansion chamber are shown in Figure 2.22 for an optical box of arbitrary length and for the GlueX design length. Two expansion chambers are used, one for the two BaBar boxes above the beam hole and one for the two boxes below the beam hole [107]. The design goal of the DIRC upgrade is to push the limits of pion-kaon separation up to momenta of $4 \mathrm{GeV} / c$. 
Figure 2.21: (Left) Schematic of the expansion volume and optical box mirrors. Cherenkov light from the BaBar boxes enter from the bottom left and is directed by the mirrors to the photosensor array at the top right. (Right) Three-dimensional rendering of the optical box coupled to two bar boxes. Figure from Reference [107].
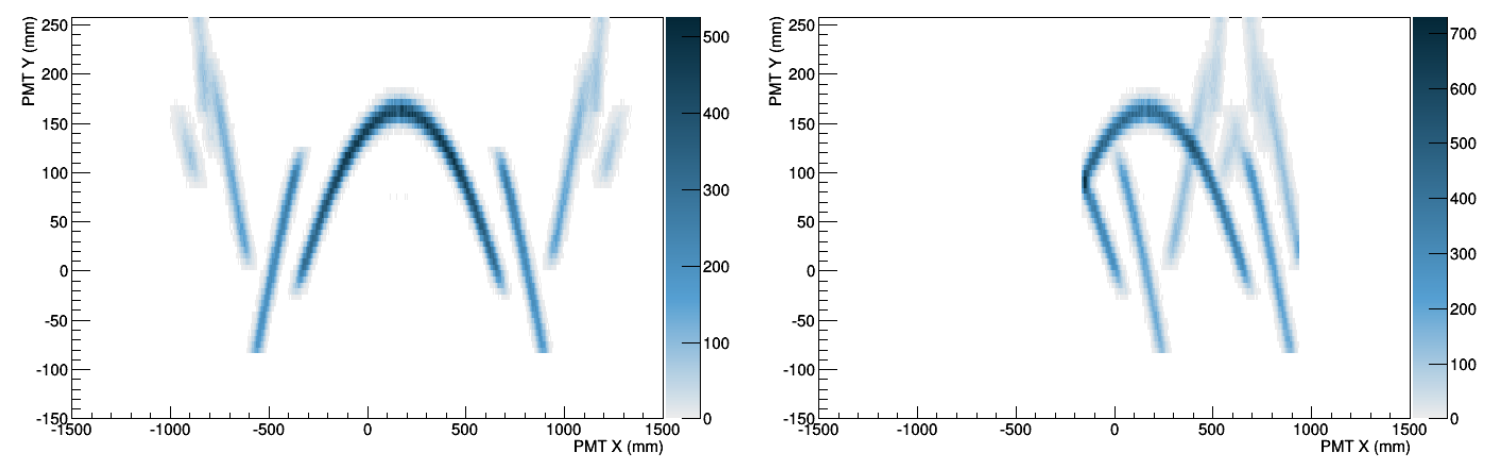

Figure 2.22: Simulated PMT photosensor patterns for normally incident charged particles and an optical box mirror setup similar to that in Figure 2.21. (Left) Optical box of arbitrary length: the entire pattern is available without reflection. (Right) $1.4 \mathrm{~m}$ long optical box, as designed for GlueX, provides the same pattern with some folding due to reflection. Figure from Reference [107]. 


\subsection{GlueX Summary}

The GlueX detector reconstructs both charged and neutral final state particles and has nearly- $4 \pi$ hermetic coverage. Precision tracking chambers allow accurate reconstruction of charged particle trajectories and interaction vertices, while calorimeters provide total energy and positional information for charged and neutral particles. Energy deposition and timing information from various subsystems provide PID capabilities, and the inclusion of the newly-installed DIRC in future run periods will enhance the detector's capability to separate charged pions and kaons. 


\section{Chapter 3}

\section{Data Analysis}

The accelerator at JLab typically runs from September through December (called 'fall') and from January through April (called 'spring'). From the spring running of 2016 until the spring running of 2018, the GlueX detector has collected $2.15 \times 10^{11}$ 'triggers.' The trigger conditions vary between and within run periods, but generally a trigger occurs and data is read out when there is a photon hit in the TAGH or TAGM (indicating a generated Bremsstrahlung beam photon) in coincidence with an energy deposition in some combination of subsystems (ST, BCAL, FCAL, etc.). The physics data set from spring of 2016 was opportunistic: detector commissioning and testing proceeded faster than expected, allowing time to take some physics data. This opportunistic data set was used for the analysis of the $2 \gamma$ final state $\left(\pi^{0}\right.$ and $\left.\eta\right)$ beam asymmetries [63]. For this, two different beam polarizations were used $\left(0^{\circ}\right.$ and $\left.90^{\circ}\right)$ in addition to an amorphous (aluminum, no photon beam polarization) radiator. Spring 2017 was the first full production run, focussed entirely on taking physics data. This data set features a factor of 2.5 more triggers than the spring 2016 run and four beam polarizations $\left(0^{\circ}, 45^{\circ}, 90^{\circ}\right.$, and $\left.135^{\circ}\right)$ along with a smaller percentage of amorphous data. Spring 2018 boasts another factor of 2 more triggers than spring 2017 with the same four beam polarizations ${ }^{1}$. During the fall of 2018 , another data set about the

\footnotetext{
${ }^{1}$ No physics triggers were collected during the fall of 2016 or the fall of 2017 due mainly to problems with the accelerator.
} 
size of the spring 2017 data set was collected that included a block of low-energy data. The low-energy data were produced using a photon beam with the coherent peak at $7 \mathrm{GeV}$ rather than the nominal $9 \mathrm{GeV}$. These data allow for studies of low-energy cross sections that would overlap with past CLAS measurements at $6 \mathrm{GeV}$ in Hall B and provide a check for systematic effects.

Before being useful for physics, data from each run period must be calibrated. The calibration process involves comparing data signals to known observables and adjusting the data or reconstruction to match those observables. For example, calibration of the BCAL SiPM photodetector gains involves reconstructing $\pi^{0}$ particles. Since the $\pi^{0}$ decays to 2 photons, the $\pi^{0}$ peak can be observed by constructing the invariant mass of two photons detected in the BCAL. Calibration constants (multiplicative factors) are then applied to the SiPM output to bring the data peak closer to the true $\pi^{0}$ mass. The process is iterated until the agreement is satisfactory. Other calibrations must be done, including calibrations on the timing of hits in the BCAL and on the attenuation of photons travelling through the scintillating fibers. Similar calibrations must be carried out for each subsystem in the GlueX detector, a process that can take several months. As such, fall 2018 data will not be available for physics analysis for a number of months, and spring 2018 calibrations are only just maturing.

Since the spring 2016 data set has comparatively low statistics (particularly for the $\eta^{\prime}$ decay channel), and because the 2018 data has yet to be fully calibrated, the data presented in this thesis includes only the spring 2017 data set, which includes about $1 \times 10^{10}$ polarized triggers split evenly over the four polarization orientations plus $8 \times 10^{9}$ amorphous triggers. In this chapter, the selection of $\eta$ and $\eta^{\prime}$ events is described, the methodology for extracting the $\Sigma$ beam asymmetry from fits to the data is detailed, and the inputs to the beam asymmetry fits are discussed. 


\subsection{Event Selection}

Event selection is done in multiple stages to ease computational requirements. Events that are unlikely to contain $\eta$ or $\eta^{\prime}$ events are removed as soon as possible to avoid large file sizes and the computationally intense processes such as the kinematic fit (see Section 3.1.2). The goal of the event selection process is to generate as clean (separated from contributions from other particles) and as pure (high signal-tobackground ratio) as possible samples of $\eta$ mesons in the decay channels of interest. These samples can then be used to investigate physics observables like cross sections or the beam asymmetries. This work focusses on the three decay channels of the $\eta$ with the highest branching fractions and the $\eta^{\prime}$ decay channel with the highest branching fraction [6]. These channels are

$$
\begin{array}{lll}
\eta \rightarrow 2 \gamma & \mathrm{BR} \approx 39.4 \% & \text { (Referred to as '2g') } \\
\eta \rightarrow \pi^{+} \pi^{-} \pi^{0} & \mathrm{BR} \approx 22.9 \% & \text { (Referred to as '3piq') } \\
\eta \rightarrow 3 \pi^{0} & \mathrm{BR} \approx 32.7 \% & \text { (Referred to as '3pi0') } \\
\eta^{\prime} \rightarrow \pi^{+} \pi^{-} \eta, \eta \rightarrow 2 \gamma & \mathrm{BR} \approx 43.4 \% \cdot 39.4 \% & \text { (Referred to as 'etaprime') }
\end{array}
$$

\subsubsection{Phase I Selection}

The first step in the event selection is to pick out combinations of particles in an event that match the desired topology, which in this context refers to the final state particles in the studied decay channels. For example, the topology of the 3piq channel is $p \pi^{+} \pi^{-} \gamma \gamma$ (the recoil proton, the charged pions, and the two photons from the $\pi^{0}$ decay). The tracking chambers may detect many charged particle tracks and the calorimeters may detect many neutral particle showers in an event, but since the topology of interest is known, events are filtered to those that contain the correct number of tracks and showers. In this analysis, 'exclusive' events are selected. 
This means that the event should contain only the recoil proton and the final state particles from the decay of the $\eta$ or $\eta^{\prime 2}$. For the analyses presented in this thesis, all particles are detected. Although selecting exclusive events can reduce the number of signal events, it reduces file size (and processing time) and increases signal purity. Multiple photons could be grouped together in one calorimeter shower if they are spatially near one another, and spurious showers could form due to photodetector noise or electromagnetic split-offs (one photon or charged particle generating multiple charged particles that are grouped into multiple showers). As such, events cannot be eliminated exclusively based on the number of charged tracks and calorimeter showers, but those with more than two or three extra of either are extremely unlikely to have been caused by an exclusive event and are cut. For the remaining events, particle combinations (referred to as 'combos') are formed. For example, for the $\eta \rightarrow \pi^{+} \pi^{-} \pi^{0}$ channel, particle combos include two positively-charged tracks (the $\pi^{+}$and the recoil proton), one negatively-charged track $\left(\pi^{-}\right)$, and two neutral showers $\left(\pi^{0} \rightarrow 2 \gamma\right)$.

Standard GlueX cuts are applied in Phase I. The first are PID cuts. The CDC is used to evaluate the energy loss of a charged track per unit length, $\mathrm{dE} / \mathrm{dx}$, which is used to separate charged particle species up to $1 \mathrm{GeV} / \mathrm{c}$ in momentum. Figure 2.12 shows an example plot from data of the $\mathrm{CDC} \mathrm{dE} / \mathrm{dx}$ versus charged track momentum for proton candidates. The loose cuts used in this phase are meant only to exclude misidentified particles. Proton candidates that lie above the red curve shown in the figure are retained by the cut. Similar cut curves are applied to electron, pion, and kaon candidates [108] if applicable.

Next, a loose cut on the invariant mass of decaying particles is applied. Those include the candidate initial particles (the photoproduced $\eta$ or $\eta^{\prime}$, here) and intermediate decays (the $\pi^{0}$ or $\eta$ decaying to $2 \gamma$ in the considered decay modes). The invariant mass of the combo particles - which is meant to reconstruct to the decaying

\footnotetext{
${ }^{2}$ An 'inclusive' event, on the other hand, would contain all these particles, plus it would be allowed to contain any number of extra tracks or showers.
} 
particle's mass - is constructed, and if it is far from the true mass of the decaying particle, the event is discarded. This cuts down on very high- or low-mass events that will enlarge file sizes and will be cut in the later phases anyway. Equation 3.1 shows this cut for decaying $\pi^{0}, \eta$, and $\eta^{\prime}$ particles.

$$
\begin{aligned}
\text { Decaying } \pi^{0}: & 0.08 \mathrm{GeV}<m_{\pi^{0}}<0.19 \mathrm{GeV} \\
\text { Decaying } \eta: & 0.35 \mathrm{GeV}<m_{\eta}<0.75 \mathrm{GeV} \\
\text { Decaying } \eta^{\prime}: & 0.60 \mathrm{GeV}<m_{\eta^{\prime}}<1.30 \mathrm{GeV}
\end{aligned}
$$

Next, a loose missing mass cut is applied that helps select exclusive events. The missing mass $(\mathrm{MM})$ is the difference in invariant mass between initial and final state particles, as defined in Equation 3.2 for the 3piq decay channel. For an exclusive event with no extra particles produced, MM should be 0 . A cut requiring $\left|\mathrm{MM}^{2}\right|<0.05 \mathrm{GeV}^{2}$ is applied. This loose cut allows for the possibility of excess showers due to electronic noise or split-offs, but cuts events that are unlikely to be exclusive events.

$$
\mathrm{MM}_{3 \text { piq }}^{2}=\left(p_{i}-p_{f}\right)^{2}=\left(p_{\gamma}^{\text {beam }}+p_{\text {proton }}^{\text {target }}-\left(p_{\text {proton }}^{\text {recil }}+p_{\pi^{+}}+p_{\pi^{-}}+p_{\gamma, 1}+p_{\gamma, 2}\right)\right)^{2}
$$

The final Phase I cut is on particle timing. This is used to determine the RF beam bunch to which a particle belongs. Since the accelerator provides bunches of beam at intervals of $4 \mathrm{~ns}$, hits in the subsystems must be matched with one another and the correct RF beam bunch. The subsystem with the best timing information is used to apply the timing cut on a particle. For example, if a charged track is matched to a hit in the TOF, no timing cut on the matched hits in the FCAL or ST is applied. The timing cuts used to identify the RF bunch corresponding to various particle species are given in Table 3.1 [108]. 


\begin{tabular}{|c|c|c|c|c|}
\hline Particle Type & ST cut & TOF cut & FCAL cut & BCAL cut \\
\hline Proton & $\left|\Delta \mathrm{t}_{\mathrm{RF}}\right|<2.5$ & $\left|\Delta \mathrm{t}_{\mathrm{RF}}\right|<0.6$ & $\left|\Delta \mathrm{t}_{\mathrm{RF}}\right|<2.0$ & $\left|\Delta \mathrm{t}_{\mathrm{RF}}\right|<1.0$ \\
\hline$\pi^{+}$ & $\left|\Delta \mathrm{t}_{\mathrm{RF}}\right|<2.5$ & $\left|\Delta \mathrm{t}_{\mathrm{RF}}\right|<0.5$ & $\left|\Delta \mathrm{t}_{\mathrm{RF}}\right|<2.0$ & $\left|\Delta \mathrm{t}_{\mathrm{RF}}\right|<1.0$ \\
\hline$\pi^{-}$ & $\left|\Delta \mathrm{t}_{\mathrm{RF}}\right|<2.5$ & $\left|\Delta \mathrm{t}_{\mathrm{RF}}\right|<0.5$ & $\left|\Delta \mathrm{t}_{\mathrm{RF}}\right|<2.0$ & $\left|\Delta \mathrm{t}_{\mathrm{RF}}\right|<1.0$ \\
\hline$\gamma$ & - & - & $\left|\Delta \mathrm{t}_{\mathrm{RF}}\right|<2.5$ & $\left|\Delta \mathrm{t}_{\mathrm{RF}}\right|<1.5$ \\
\hline
\end{tabular}

Table 3.1: Selection cuts on $\Delta \mathrm{t}_{\mathrm{RF}}$ for various particles in the ST, TOF, BCAL, and FCAL. All times are in ns.

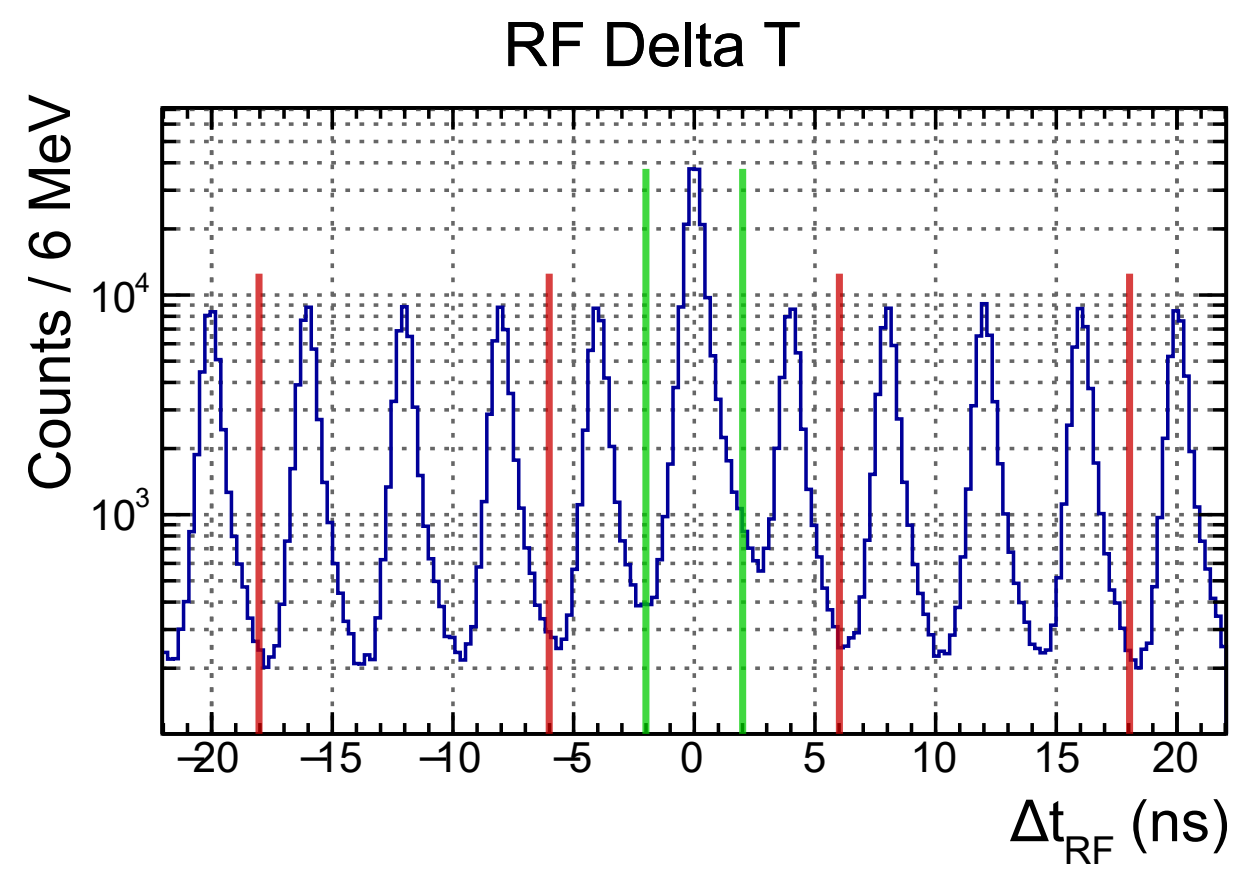

Figure 3.1: RF $\Delta$ t distribution. The central peak between the green delimiting lines contains the real photoproduction events and some contribution from accidentals. The smaller peaks are from accidental events. The six small peaks between the two sets of red delimiting lines are the peaks used for accidental subtraction, as described in Section 3.2.1 (colour online).

Figure 3.1 shows a sample $\Delta \mathrm{t}_{R F}$ distribution obtained in data. The large central peak contains the real photoproduction events, while the smaller peaks at intervals of 4 ns contain accidentally-tagged events ('accidentals'), those with subsystem hits matched to other RF beam bunches. However, the central peak also contains contributions from accidentals. In the analyses herein, five accidental RF bunches on either side of the central peak are kept. The effects of the accidentals under the 
central peak can be subtracted by constructing distributions with only events in the accidental peaks, as detailed in Section 3.2.1.

\subsubsection{Phase II Selection}

Phase II selection consists of a kinematic fit (KinFit). The KinFit is a tool that combines measured and unmeasured quantities (particle positions, momenta, and energies) with a set of constraints to produce event/decay vertices and more accurate estimations of the measured quantities. For a kinematic fit to an exclusive event, all final state particle momenta and energies are measured. The fit is a $\chi^{2}$ minimization, which tests fit quantities (variations on each measured quantity) to obtain the best $\chi^{2}$ possible. The $\chi^{2}$ is defined as in Equation 3.3 [109].

$$
\chi^{2}=\sum_{k} \frac{\left(y_{k}^{\mathrm{fit}}-y_{k}^{\mathrm{meas}}\right)^{2}}{\sigma_{k}^{2}}
$$

Here, the $y_{k}$ are the measured and fit quantities $(x, y$, and $z$ positions and momenta) and the $\sigma_{k}$ are the Gaussian measurement errors on $y_{k}{ }^{3}$. Trivially, the minimum of this $\chi^{2}$ equation is when $y_{k}^{\text {fit }}=y_{k}^{\text {meas }}$, so that constraints must be considered. The main constraints are conservation of energy and momentum. In the assumed exclusive event, measured values may not conserve these quantities because of measurement resolutions, missing or misidentified particles, etc., but the fit values are required to conserve energy and momentum. In addition, certain particles in the combo must come from the same vertices. The initially produced particles, plus the recoil proton, must come from the interaction vertex and any final state particles that come from an intermediate particle decay must come from a decay vertex. Another common constraint is on the decay mass of intermediate particles. For example, the two photons in the 3piq final state are meant to come from the decay of a $\pi^{0}$, so the

\footnotetext{
${ }^{3}$ These errors are encoded in the 'covariance matrix,' which includes information about the uncertainties on all measured quantities and their correlations with one another.
} 
fit puts a constraint on the fit that the $2 \gamma$ invariant mass must be exactly the true $\pi^{0}$ mass. Then, the $\chi^{2}$ minimization becomes a problem of minimizing Equation 3.3 by varying the $y_{k}^{\text {fit }}$ whilst simultaneously satisfying all constraints ${ }^{4}$.

If the kinematic fit does not converge, the particle combo is discarded. If the kinematic fit does converge, the minimized $\chi^{2}$ is converted to a 'confidence level' (CL), the integral of a probability density function. It is a number between 0 and 1 , representing the probability that the fit's $\chi^{2}$ is less than a real-valued random variable. In the absence of background, the CL distribution should be flat, such that a cut requiring the CL to be greater than $5 \%$ will remove $5 \%$ of the signal events. In reality, background events and those with poorly measured input quantities tend to collect near a CL of 0 . As such, a cut on low confidence levels is an extremely powerful way to eliminate background and other unwanted events while losing very few signal events.

The best CL cut to use is not an obvious choice. A very loose cut-CL $>10^{-8}$, for instance - may not eliminate enough background events to allow for a consistent physics extraction. A relatively tight cut $-\mathrm{CL}>10^{-1}$, say-will provide a very pure signal peak, but will also remove about $10 \%$ of the signal. The best cut to use is based on a balance between statistics and purity. For the analyses in this thesis, a study was conducted (which is detailed in Section 5.1) to identify reasonable CL cuts to use for each analysis. Based on these studies, CL $>10^{-5}$ is the cut used in all four decay channels.

\subsubsection{Phase III Selection}

An event vertex is defined by the charged tracks in an event with assistance from the kinematic fit. For exclusive events, all charged tracks are required to come from a common vertex inside the target volume. The center of the target lies at a

\footnotetext{
${ }^{4}$ This is done using the Lagrange multiplier method, as detailed in Reference [109].
} 
global z-position (along the beam line) of $65 \mathrm{~cm}$ and the target vessel is $30 \mathrm{~cm}$ long. To eliminate edge effects ${ }^{5}$, a cut in the z-position of the kinematically-fit vertex is placed between $52 \mathrm{~cm}$ and $78 \mathrm{~cm}$. The target is on average $2 \mathrm{~cm}$ in diameter (see Section 2.2.1.5), so the vertex radial position is required to be less than $1.0 \mathrm{~cm}$ from the z-axis. These cuts are shown in Figure 3.2.
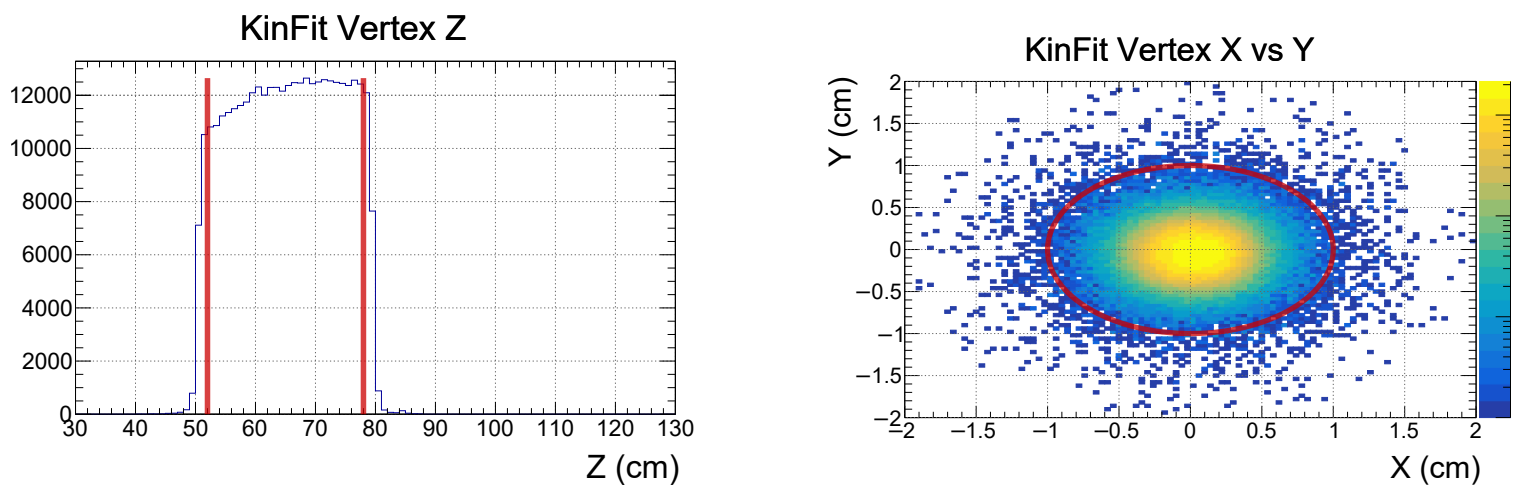

Figure 3.2: The z-position of the kinematically-fit vertex (left) and the x-position versus y-position of the kinematically-fit vertex (right). The red delimiting lines indicate the cut limits (colour online).

Detector edge effects must be understood well in order to accurately reconstruct photons. Neutral showers in the FCAL around the beam hole and near the FCALBCAL gap are often partial showers due to escaping or undetected energy, which can render calibration or reconstruction in these regions difficult or unreliable. Fiducial cuts $^{6}$ are implemented that remove particle combos with reconstructed photons in either region, as shown in Figure 3.3. For the BCAL, this effectively corresponds to a $\mathrm{z}$-position cut. The BCAL occupies the region between $\mathrm{z}=17 \mathrm{~cm}$ and $\mathrm{z}=407 \mathrm{~cm}$ in the global coordinate frame, and the fiducial cut removes events with BCAL showers reconstructed at $\mathrm{z}>380 \mathrm{~cm}$. For the FCAL, a radial position cut is used. There,

\footnotetext{
${ }^{5}$ The walls of the target vessel consist of materials other than hydrogen, and interactions of beam photons with these materials can result in more complicated reactions and final states.

${ }^{6}$ Typically obtained from simulation studies, some spatial parts of a detector (especially areas near the edges) offer decreased sensitivity, excess background, poor reconstruction, etc. The 'fiducial volume' of a detector is the rest of the detector, where sensitivity and reconstruction are understood and reliable.
} 
fiducial cuts are placed to remove events with photons reconstructed in the innermost few layers and outermost few layers of lead-glass scintillator blocks, as showers reconstructed in these regions are likely to be missing energy and are thus likely to be reconstructed incorrectly. This corresponds to removing events with radial FCAL shower positions of $\mathrm{r}<25 \mathrm{~cm}$ and $\mathrm{r}>100 \mathrm{~cm}$. Similarly, recoil proton reconstruction suffers at low momentum because of a lack of tracking information from the CDC. A fiducial cut on the proton momentum requires that $P_{\text {recoil }}>0.25 \mathrm{GeV}$.

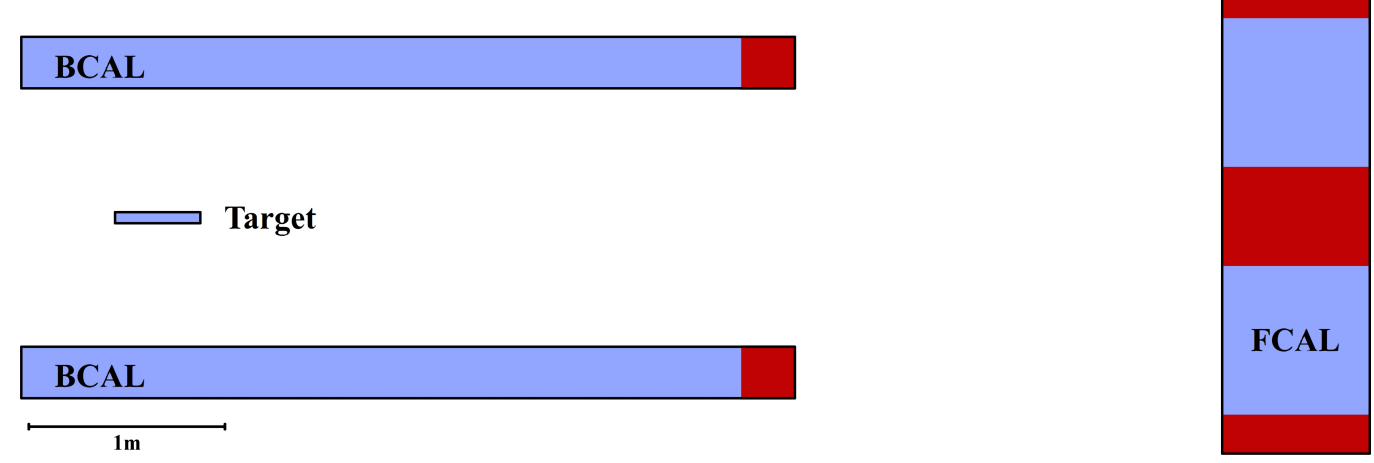

Figure 3.3: A side-view of the GlueX detector showing only the BCAL and FCAL. Fiducial cuts (shown as red regions) are placed around the beam hole in the FCAL and around the FCAL-BCAL gap where reconstruction or calibration can be difficult or unreliable (colour online).

As mentioned, the kinematic fit constrains the intermediate decay particle's mass. If the measured decay particle's mass is rather far away from the actual meson mass, the fit's confidence level should be small, or the reconstructed combo invariant mass may be pulled outside of the $\eta$ or $\eta^{\prime}$ peak region. However, it is useful to remove obvious background with a cut where feasible rather than relying on the kinematic fit to perfectly identify all background. Figure 3.4 shows plots of the reconstructed combo invariant mass versus the measured diphoton mass for the 3piq and etaprime decay channels. There are some clear outliers in the $2 \gamma$ mass spectrum in both plots that produce the desired combo invariant mass after the kinematic fit, but that are not convincingly close to the desired $2 \gamma$ mass. Tighter cuts are placed on the 
intermediate decaying $\eta$ mass (between 0.46 and $0.62 \mathrm{GeV}$ ) and $\pi^{0}$ mass (between 0.105 and $0.165 \mathrm{GeV}$ ) as depicted in the figure ${ }^{7}$.
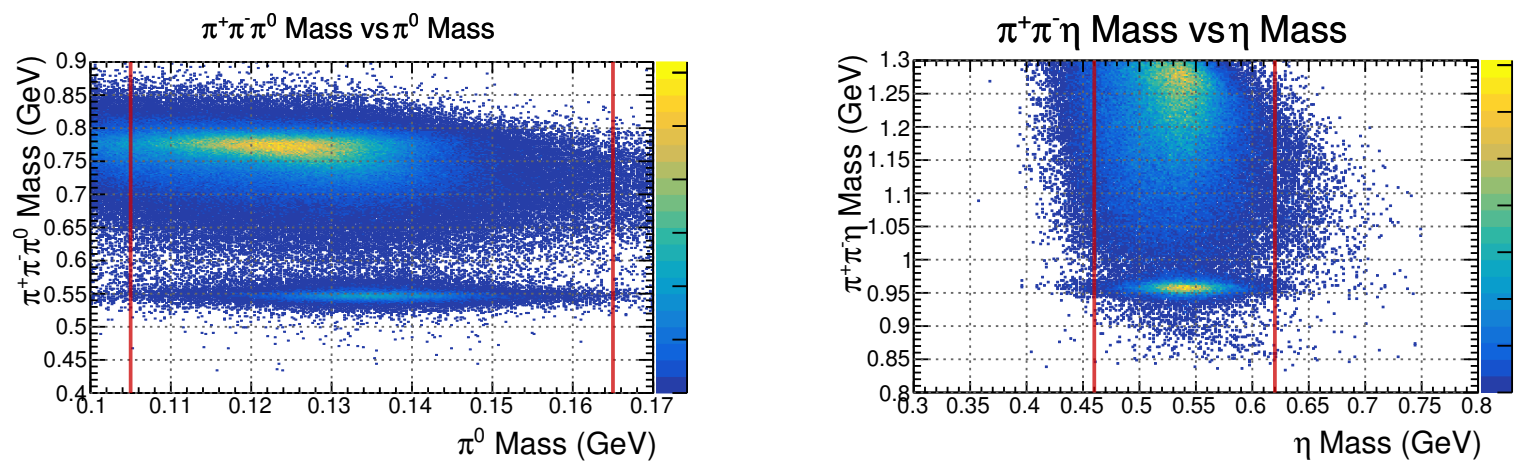

Figure 3.4: KinFit $\pi^{+} \pi^{-} \pi^{0}$ mass vs. measured $\pi^{0} \rightarrow 2 \gamma$ mass (left) and KinFit $\pi^{+} \pi^{-} \eta$ mass vs. measured $\eta \rightarrow 2 \gamma$ mass (right). The red delimiting lines indicate the cut limits on the $2 \gamma$ masses (colour online).

Lastly, for the $\Sigma$ beam asymmetry analyses, a high beam polarization is necessary. The beam energy is restricted to be between $8.2 \mathrm{GeV}$ and $8.8 \mathrm{GeV}$, the coherent Bremsstrahlung peak, where the degree of polarization is highest. Figure 3.5 shows the combo invariant mass spectra for the three $\eta$ decay channels and the $\eta^{\prime}$ decay channel after various cuts in the event selection.

A list of all event selection cuts discussed in this chapter can be found in Appendix A.

The black spectra in Figure 3.5 show the invariant mass after all Phase I cuts and with a converging kinematic fit $(\mathrm{CL}>0)$. The fiducial cuts (neutral showers near the edges of the calorimeters and low-momentum protons) and the beam energy cut (which selects the coherent peak) remove approximately $90 \%$ of the events. The remaining cuts on the vertex position inside the target, the CL, and the intermediate decay particle mass remove around $32 \%$ of the remaining events (on average, over the four channels). As seen in the figure, these cuts do not remove a significant amount of combos in the peak region (the corresponding histograms are nearly indistinguishable

\footnotetext{
${ }^{7}$ This decaying $\pi^{0}$ mass cut is also used for the 3pi0 decay channel.
} 
$\eta$ Invariant Mass

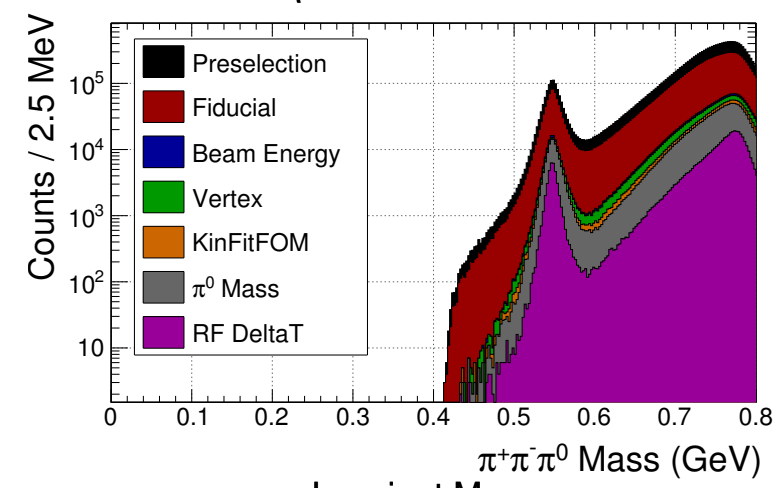

$\eta$ Invariant Mass

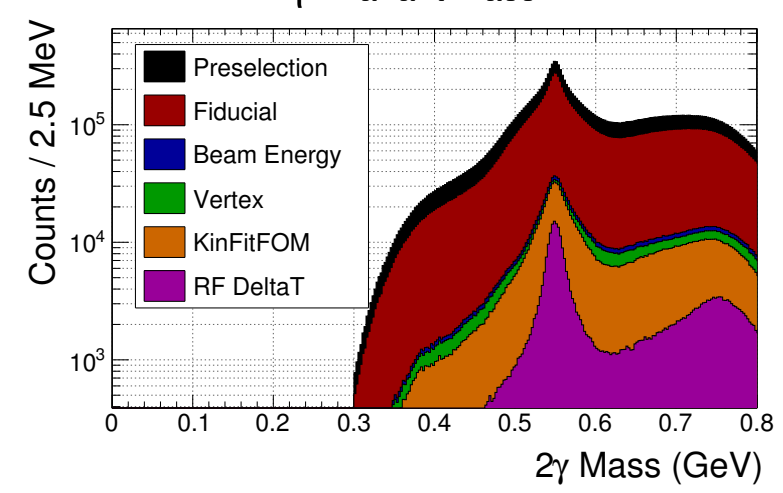

$\eta$ Invariant Mass

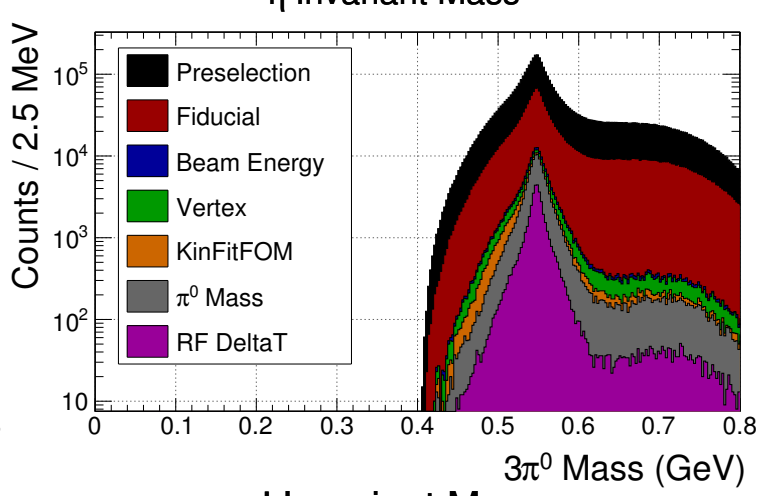

$\eta^{\prime}$ Invariant Mass

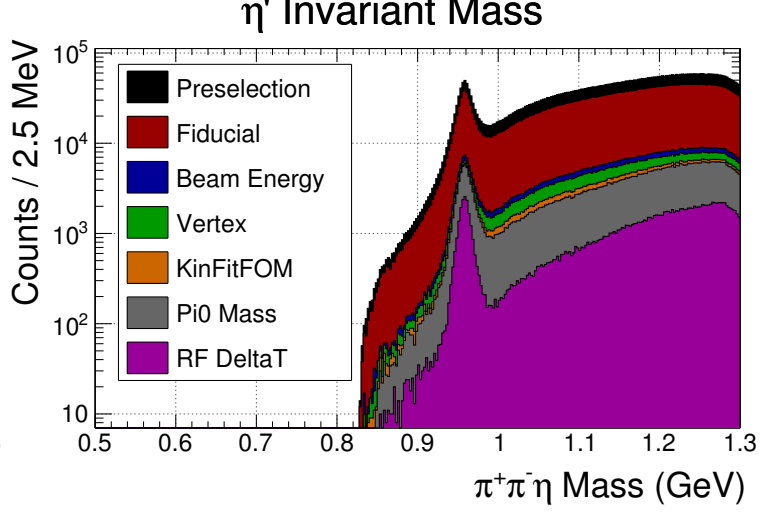

Figure 3.5: Invariant mass spectra of the $\eta$ for the 3piq (top left), 3pi0 (top right), and $2 \mathrm{~g}$ (bottom left) decay modes, and of the $\eta^{\prime}$ (bottom right). The effects on the mass spectra of various cuts are shown (colour online).

in the peak region), but the reduction in background is noticeable. Lastly, the RF cut selects the central RF peak.

The $\eta$ or $\eta^{\prime}$ events can be filtered for further analysis by selecting events in the 'peak region' of these spectra. The peak region is chosen to encompass the majority of the events in the particle peak and as few as possible background events. Generally, the peak region is defined in some mathematical way so there is consistency between analyses. Here, the peak region is defined by the width of a Gaussian fit. The Gaussian (Normal) distribution, shown in Figure 3.6, has three parameters: the amplitude $(A)$, the mean $(\mu)$, and the width $(\sigma)$. It is widely used to fit statistical data as a method of finding the mean or resolution of a data set. The Gaussian distribution has the property that $95 \%$ of the area under the Gaussian curve is contained in a 
range of $\pm 2 \sigma$ from the mean. In terms of a fit to a mass spectrum, then, $\mu$ should be the true mass of the particle, and a $2 \sigma$ range around $\mu$ will include about $95 \%$ of the particle signal events. The peak region for each decay channel, then, is defined as $\mu \pm 2 \sigma$.

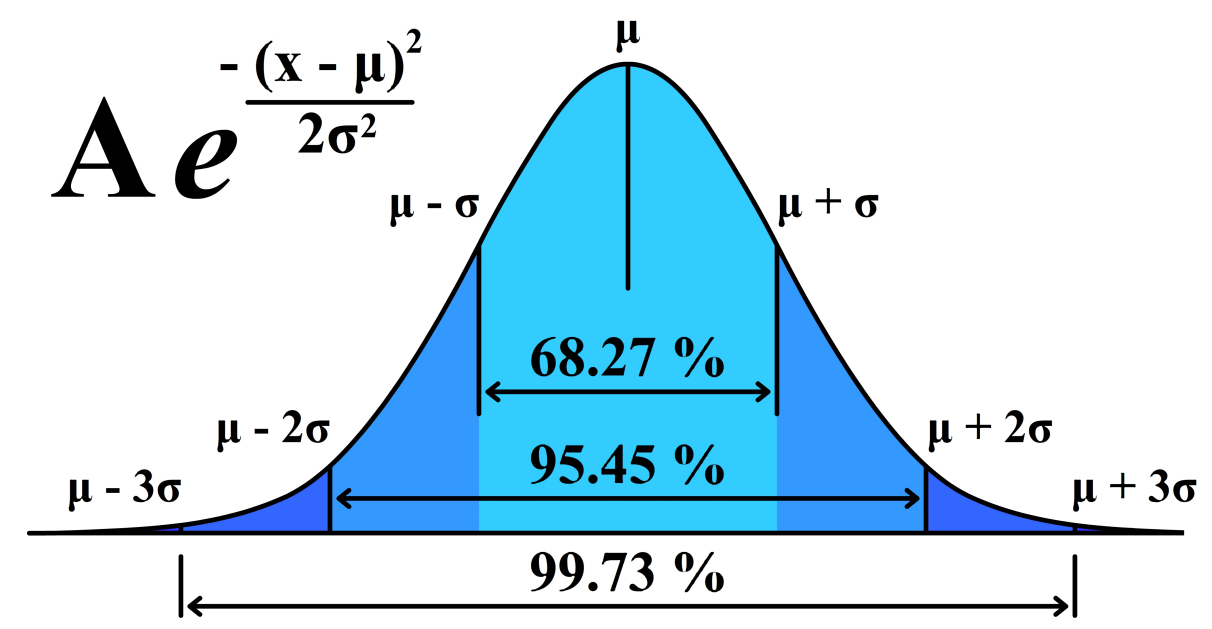

Figure 3.6: The Gaussian distribution is a symmetric function around some mean, $\mu$, with variance $\sigma^{2}$ and amplitude A. The integral under the curve in the range $\mu \pm \sigma$, $\mu \pm 2 \sigma$, and $\mu \pm 3 \sigma$ are displayed, along with the functional form.

Fits to the mass distributions are comprised of two Gaussian distributions to describe the peak plus an exponential function to describe the background. The 3pi0 decay channel has very little background in the $\eta$ mass region after event selection, so the exponential is not included in mass fits to the 3pi0 channel. However, two Gaussians alone do not adequately fit the data because of the very small background contribution. The fit to the 3 pi0 mass spectrum thus features a small constant term (on the order of 30 counts) in place of the background exponential. The double Gaussian is used to describe the peak because events will see some number of showers in the BCAL and the rest in the FCAL, but these two subsystems have different energy resolutions. The mass distributions are thus best described by a sum of two Gaussians with the same mean $(\mu)$ but different widths $\left(\sigma_{1}, \sigma_{2}\right)$ and amplitudes $\left(A_{1}, A_{2}\right)$. The peak region is then defined as $\mu \pm 2 \sigma_{L}$, where $\sigma_{L}$ is the larger of the two Gaussian 

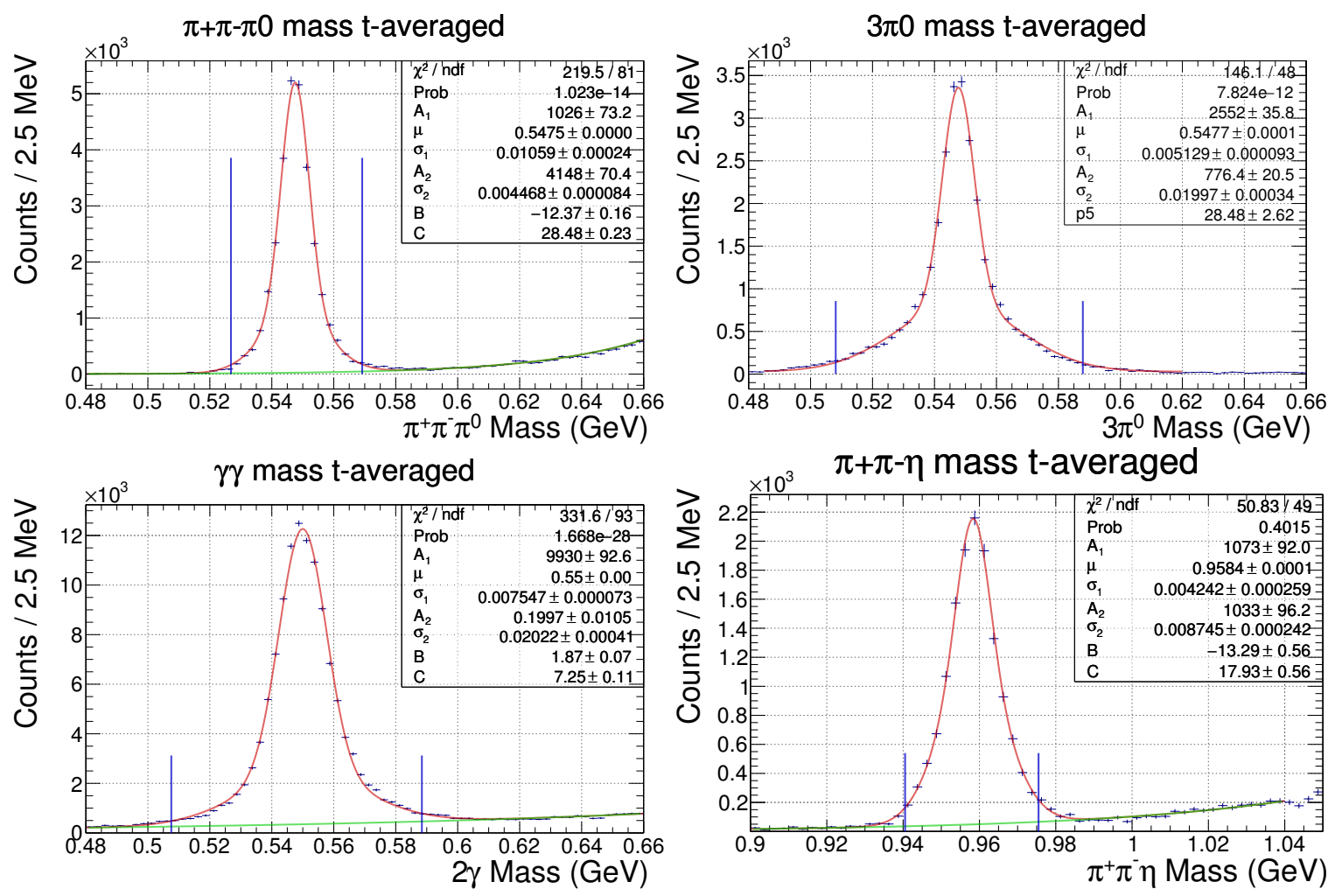

Figure 3.7: Fits to the invariant mass spectra of the $\eta$ for the 3piq (top left), 3pi0 (top right), and $2 \mathrm{~g}$ (bottom left) decay modes, and of the $\eta^{\prime}$ (bottom right), to Equation 3.4. The exponential component of the fit function is replaced by a small constant term for the 3 pi0 decay mode (omitted from the fit statistics). The peak region used to select signal events for further analysis is displayed in each plot. These spectra are integrated over the entire range of $t$ studied, referred to as ' $t$-averaged.'

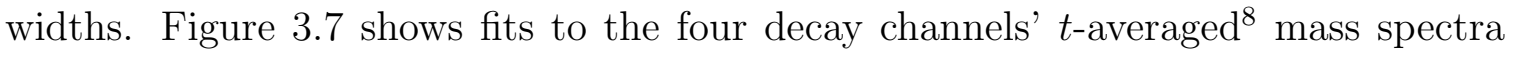
along with the peak regions, and the fit function used is expressed in Equation 3.4. Generally, a fit that accurately describes the data (with reasonable statistical errors) should have a $\chi^{2}$ per number of degrees of freedom $\left(\chi^{2} / n d f\right)$ of 1 . In each of the three $\eta$ fits, the $\chi^{2} / n d f$ is much larger than 1 , implying that the fit function used may not describe the data perfectly. However, these fits are only used to estimate the width of the distribution (to define the peak region) and the amount of background under the peak (to calculate $f$, the 'dilution factor' (see Section 3.2.2)). Since the fits shown

\footnotetext{
${ }^{8}$ ' $t$-averaged' throughout this thesis refers to a result obtained using data from all bins in $t$ used, $0.1 \mathrm{GeV}^{2}<-t<1.2 \mathrm{GeV}^{2}$. The choice of binning will be discussed in Section 3.2.
} 
in the figure allow these estimations to be done, no major effort was made toward improving the fit metrics.

$$
f(x)=A_{1} e^{-\frac{(x-\mu)^{2}}{2 \sigma_{1}^{2}}}+A_{2} e^{-\frac{(x-\mu)^{2}}{2 \sigma_{2}^{2}}}+e^{B+C x}
$$

\subsection{Beam Asymmetry Analysis Methodology}

After all event selection cuts, the $\Sigma$ beam asymmetry can be extracted from the data. This is done through the use of Equation 1.8, which gives the polarized cross section as a function of the unpolarized cross section, the polarization of the beam, $P_{\gamma}$, and the azimuthal angle between the reaction plane and the polarization direction of the beam $\left(\Phi=\phi-\phi_{\gamma}\right)$. In principle, fitting the experimentally measured polarized cross section to this equation, where the unpolarized cross section and the beam polarization are known, will immediately give the $\Sigma$ beam asymmetry. However, the simple distributions that are created are not cross sections, but yields ${ }^{9}$. These are simply the number of $\eta$ or $\eta^{\prime}$ mesons as functions of $\phi$, the azimuthal angle of the reaction plane, and $t$, the four-momentum transfer to the proton squared, obtained by selecting combos in the $\eta$ or $\eta^{\prime}$ peak region of the mass spectra after event selection for each decay channel and plotting $\phi$ of the combo proton versus $t$. Transition between a yield and a cross section involves the acceptance of the detector, which is a measure of how sensitive a detector is to detection of an event dependent on angles, energies, etc. Equation 1.8 becomes Equation 3.5, in terms of yield.

$$
Y_{\gamma}(\phi) \sim N_{\gamma}\left[\sigma_{0} A(\phi)\left(1+P_{\gamma} \Sigma \cos \left(2\left(\phi-\phi_{\gamma}-\phi_{0}\right)\right)\right)\right]
$$

Here, $Y_{\gamma}(\phi)$ is the $\phi$-dependent yield from a photon beam polarized at an angle

\footnotetext{
${ }^{9}$ As mentioned in Section 1.6, cross section measurements require detailed, accurate MC simulation acceptance studies. Work on these acceptance studies for GlueX is underway, but has not yet reached a mature state.
} 
$\phi_{\gamma}$ with polarization $P_{\gamma}, N_{\gamma}$ is the number of photons with polarization angle $\phi_{\gamma}$ incident on the target, and $A(\phi)$ is the $\phi$-dependent acceptance of the detector. Since the GlueX detector is azimuthally symmetric, the acceptance is not expected to have a dependence on $\phi$, but problems with calibration or extra material (cables, electronics boards, etc.) in certain $\phi$ regions may result in altered acceptance. $\phi_{0}$ is a small phase offset (on the order of a few degrees) to account for misalignments of the diamond radiator and detector. In order to eliminate both the unpolarized cross section and the acceptance from the expression, two data sets with polarizations perpendicular to each other can be used. The first, called 'PARA,' is with a beam polarized at $\phi_{\gamma}=0^{\circ}$ (historically so-called because the beam polarization is parallel to the lab floor). The second, called 'PERP,' is with a beam polarized at $\phi_{\gamma}=90^{\circ}$. Then, the PARA and PERP yields are as in Equation 3.6.

$$
\begin{gathered}
Y_{0^{\circ}}=Y_{\|}(\phi) \sim N_{\|}\left[\sigma_{0} A(\phi)\left(1-P_{\|} \Sigma \cos \left(2\left(\phi-\phi_{0}\right)\right)\right)\right] \\
Y_{90^{\circ}}=Y_{\perp}(\phi) \sim N_{\perp}\left[\sigma_{0} A(\phi)\left(1+P_{\perp} \Sigma \cos \left(2\left(\phi-\phi_{0}\right)\right)\right)\right]
\end{gathered}
$$

The yield asymmetry is then formed between the PARA and PERP yields to eliminate $\sigma_{0}$ and $A(\phi)$. This is the difference between the two yields normalized to the sum: $f(\phi)=\frac{Y_{\perp}(\phi)-F_{R} Y_{\|}(\phi)}{Y_{\perp}(\phi)+F_{R} Y_{\|}(\phi)}$, shown in Equation 3.7. Here, $F_{R}=\frac{N_{\perp}}{N_{\|}}$is a normalization factor determined by integrating the photon flux for PARA and PERP orientations in the beam energy region of interest (see Section 3.3.1).

$$
f(\phi)=\frac{\left(P_{\perp}+P_{\|}\right) \Sigma \cos \left(2\left(\phi-\phi_{0}\right)\right)}{2+\left(P_{\perp}-P_{\|}\right) \Sigma \cos \left(2\left(\phi-\phi_{0}\right)\right)}
$$

In order to extract the beam asymmetry, $\Sigma$, from a fit of this function to data, values for $P_{\perp}, P_{\|}, \phi_{0}$, and $F_{R}$ must be known. Measurements of these parameters are discussed in Section 3.3.

A measurement of the fit parameter, $\Sigma$, along with an error on the fit parameter, 
$\delta_{\Sigma}$, is done in this way using the $0^{\circ}$ (PARA) and $90^{\circ}$ (PERP) polarization data. This set of perpendicularly-polarized data is referred to as '0/90.' Similarly, the $135^{\circ}$ (PARA) and $45^{\circ}$ (PERP) data forms a second set of perpendicularly-polarized data, referred to as '45/135.' The decision to collect two sets of perpendicularlypolarized data was made to study possible systematic effects of the diamond radiator orientation. As such, these two data sets are analyzed independently, then an errorweighted average is performed to obtain final results. Furthermore, the yields are split up into bins of $t$ and the asymmetry analysis is carried out over the data in each bin. The number of $t$ bins used is limited by statistics, particularly at higher $t$, and the bins are defined such that each bin contains roughly the same number of events. Thus, for the analyses in this thesis, four bins of $t$ are used (given in Table 3.2), and the binning is the same for each of the decay channels. The results are then $\Sigma$ as functions of $t$ for the four decay channels, which can then be compared to theory predictions and past measurements.

\begin{tabular}{|c||c|}
\hline Bin & $t$ Range $\left(\mathrm{GeV}^{2}\right)$ \\
\hline \hline$t$-averaged & $0.1<-t<1.2$ \\
1 & $0.1<-t<0.3$ \\
2 & $0.3<-t<0.5$ \\
3 & $0.5<-t<0.7$ \\
4 & $0.7<-t<1.2$ \\
\hline
\end{tabular}

Table 3.2: The ranges in $t$ used to define each $t$ bin. The $t$-averaged results are those obtained using the entire range in $t$ spanned by the four bins.

\subsubsection{Accidental Subtraction}

As mentioned in Section 3.1.1, events in the central RF peak contain contributions from accidentally-tagged events. To correct for this, each distribution made from data (mass spectra, yields as functions of $\phi$ and $t$, etc.) for events in the central RF peak $(|\Delta \mathrm{t}|<2.004 \mathrm{~ns})$, shown by green delimiting lines in Figure 3.1, is also made for 
events in some number of accidental peaks $\left(6.012 \mathrm{~ns}<|\Delta \mathrm{t}|<18.036 \mathrm{~ns}^{10}\right)$, shown by red delimiting lines in the same figure. The distributions made using events in the accidental peaks are scaled by a factor of $\frac{1}{6}$ (since they represent the contributions from 6 accidental RF peaks) and subtracted from the central peak distributions. In this way, the effect of the accidentals under the central RF peak is properly accounted for. All mass spectra and yield distributions presented in this thesis are to be understood as being accidentals-subtracted in this way unless otherwise stated.

\subsubsection{Side-Band Asymmetry Correction}

For some decay channels, there is substantial background under the $\eta$ or $\eta^{\prime}$ peak. The beam asymmetries for the particle and the background may be different, so by only measuring the asymmetry for events under the particle peak, the result can be diluted. To account for the possibility of a different background asymmetry, a mass side-band correction is performed.

For this, the asymmetry with all events in the peak region $( \pm 2 \sigma$ from the peak mass value) is measured as $\Sigma_{\text {peak }} \pm \delta_{\Sigma_{\text {peak }}}$. Then, the asymmetry using events in a mass side-band $(\mathrm{SB})$ region of the distribution is measured as $\Sigma_{\mathrm{SB}} \pm \delta_{\Sigma_{\mathrm{SB}}}$. The SB region chosen in each case is a mass window just above the particle peak in mass that contains enough statistics to perform an asymmetry analysis in bins of $t$. It is assumed that the asymmetry in the SB region is the same as the asymmetry of the background events under the peak ${ }^{11}$. Finally, the 'dilution factor,' $f=\frac{\text { Background }}{\text { Signal+Background }}$, in the peak region is measured. The double Gaussian plus background fits to the mass spectra (Figure 3.7) can be used to estimate the dilution factor with an error, $\delta_{f}$, by taking the integral of the background part of the fitted function over the peak region divided by the integral of the mass histogram over the same region. The errors on these two

\footnotetext{
${ }^{10}$ Since the central RF peak leaks into the two adjacent accidental peaks, these two peaks are omitted from the accidental distributions.

${ }^{11}$ This assumption is tested for all relevant decay channels in Section 5.2.1.
} 
integrals are just the square roots of the number of counts, and the statistical error on $f$ can be calculated in the usual way. Then, the corrected asymmetry, $\Sigma_{\mathrm{COR}}$, and the error on the corrected asymmetry are given in Equation 3.8.

$$
\begin{aligned}
& \Sigma_{\mathrm{COR}}=\frac{\Sigma_{\text {peak }}-f \Sigma_{\mathrm{SB}}}{1-f} \\
& \delta_{\Sigma_{\mathrm{COR}}}=\sqrt{\left(\frac{\delta_{\Sigma_{\text {peak }}}}{1-f}\right)^{2}+\left(\frac{\delta_{\Sigma_{\mathrm{SB}}} f}{1-f}\right)^{2}+\left(\frac{\delta_{f}\left(\Sigma_{\text {peak }}-\Sigma_{\mathrm{SB}}\right)}{(1-f)^{2}}\right)^{2}}
\end{aligned}
$$

The side-band region chosen depends on the decay channel. For example, the dominant background in the 3piq channel for the $\eta$ is from the similar decay of the $\omega(782)$ meson. As such, the $\omega$ peak is included in the side-band region to extract $\Sigma_{\mathrm{SB}}$ since almost all the background events under the $\eta$ peak will be $\omega$ events. Again, this assumes the asymmetry of this $\omega$ background is not mass-dependent. Similarly, the primary background in the $2 \mathrm{~g}$ channel for the $\eta$ is from the $\omega$ decaying to $\pi^{0} \gamma$ with a missing photon [63], and the $\omega$ peak is again included in the side-band region. In contrast, the nature of the background contributions in the $\eta^{\prime}$ decay channel is not known. The side-band region in this channel is selected to encompass enough events for a reasonable asymmetry fit while staying low enough in mass to exclude the $f_{1}(1270)$ particle peak, since the $f_{1}$ event distribution is not likely to extend under the $\eta^{\prime}$ peak.

Mass spectra with extracted dilution factors and choices of side-band regions will be shown in the relevant sections of Chapter 4 .

\subsection{Asymmetry Fit Parameters}

To extract $\Sigma$ from a fit to data with the function in Equation 3.7, $\Sigma$ should be the only free parameter in the equation. The remaining parameters, $P_{\perp}, P_{\|}, \phi_{0}$, and 
$F_{R}$, are fixed ${ }^{12}$ by the results of various studies described in this section.

\subsubsection{Flux Ratio}

The integrated photon flux for a given set of runs is determined using the PS hits in coincidence with a hit in the photon tagger. Accidentally tagged photons are subtracted in the same way as described in Section 3.2.1, where $\Delta \mathrm{t}$ in this case is the time difference between the PS pair and the tagger hit. The accidental-corrected integrated flux with this tagger coincidence is referred to as the tagged flux, which is shown in Fig 2.8, integrated over all spring 2017 runs used in the analyses herein. The ratio of yields in the coherent peak energy range, $8.2<E_{\gamma}<8.8 \mathrm{GeV}$, between PERP and PARA data provides a measure of the flux ratio $\left(F_{R}\right)$ for the $0 / 90$ and 45/135 data sets. Table 3.3 shows the PARA and PERP integrated flux and the resulting flux ratio for both the $0 / 90$ and $45 / 135$ data sets. The $F_{R}$ values have negligible statistical uncertainties due to the high PS counting rate and coinciding large statistics. The systematic error on the determination of the flux ratio, estimated at $\pm 5 \%$, is considered in Section 5.2.5.

\begin{tabular}{|c||c|c|c|}
\hline Data Set & PARA Integrated Flux & PERP Integrated Flux & $F_{R}$ \\
\hline \hline $0 / 90$ & $4.188001 \times 10^{12}$ & $4.346818 \times 10^{12}$ & 1.037922 \\
$45 / 135$ & $4.095013 \times 10^{12}$ & $4.076065 \times 10^{12}$ & 0.995373 \\
\hline
\end{tabular}

Table 3.3: Integrated PS Flux and Flux Ratios for the two data sets.

\subsubsection{Beam Polarization}

As discussed in Section 2.2.1.3, the polarization of the photon beam is measured by the TPOL. Figure 2.6 shows the polarization of the photon beam in all four polarization orientations in bins of beam energy. The polarizations used in the yield asymmetry fit function, $P_{\perp}$ and $P_{\|}$for both the $0 / 90$ and $45 / 135$ data sets, are best

\footnotetext{
${ }^{12} \mathrm{~A}$ 'fixed' parameter means one that is assigned a numerical value based on some study and treated as a constant in the fit.
} 
calculated as weighted averages of the TPOL results in the coherent peak energy range $(8.2 \mathrm{GeV}$ to $8.8 \mathrm{GeV})$, with the weights being the number of events in the peak mass region in each of the three beam energy bins which cover this energy range. Since the distribution of events versus beam energy may not be the same in each decay mode - because of differences in acceptance and detection efficiency for different topologies (6 photon versus 2 photon decay, neutral particles versus charged particles, etc.) - the weighted averages for the four polarizations must be computed for each decay channel separately, giving 16 different polarizations.

Although the weighted averages for each decay channel have slightly differing values, the errors on these quantities are all nearly identical. The absolute error on the weighted average of polarizations for a given decay channel, where the weights used are the number of events in the mass peak region in each beam energy bin for that decay channel, is given by Equation 3.9.

$$
\delta_{P_{a}}=\frac{\sqrt{N_{1}^{2} \delta_{P_{1}^{a}}^{2}+N_{2}^{2} \delta_{P_{2}^{a}}^{2}+N_{3}^{2} \delta_{P_{3}^{a}}^{2}}}{N_{1}+N_{2}+N_{3}}
$$

Here, $N_{n}(n=1,2,3)$ are the number of events in the peak mass region in the three beam energy bins, $P_{n}^{a}$ are the TPOL polarization measurements in those beam energy bins for a particular diamond orientation, $a$, and $\delta_{P_{n}^{a}}$ are the statistical errors of those polarization measurements.

For each of the 16 weighted averages calculated, the absolute error is $\delta_{P_{a}} \approx 0.01$. Since each weighted average is around $35 \%$, the relative error on each polarization is $\frac{\delta_{P_{a}}}{P_{a}} \approx 0.03$. Since a change in the magnitude of the polarizations will directly effect the resulting $\Sigma$ beam asymmetry by shifting all measured asymmetries to higher or lower values uniformly, the errors on the polarizations contribute to the 'normalization uncertainty' on beam asymmetry results, an uncertainty on the overall scale of $\Sigma$ measurements. In addition to the statistical errors, systematic errors on the 
TPOL measurements also contribute to the normalization uncertainty. The relative systematic uncertainty on the measurement of the analyzing power with the TPOL is estimated to be $1.5 \%$ [91]. Finally, because the polarizations from all four orientations are used to generate the final asymmetry (the weighted average of the 0/90 and $45 / 135$ asymmetry results), the statistical contribution to the normalization uncertainty is the statistical error of one polarization measurement scaled by the number of polarization measurements used. The normalization uncertainty on the average of the $0 / 90$ and $45 / 135$ results is then $\sqrt{0.03^{2} / 4+0.015^{2}}=2.1 \%$.

0/90 $\eta$ Asymmetry t-averaged

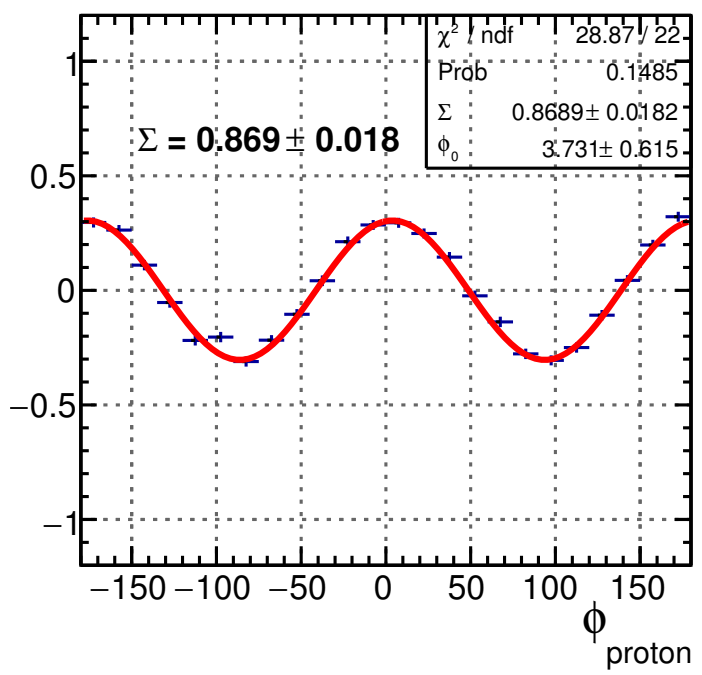

45/135 $\eta$ Asymmetry t-averaged

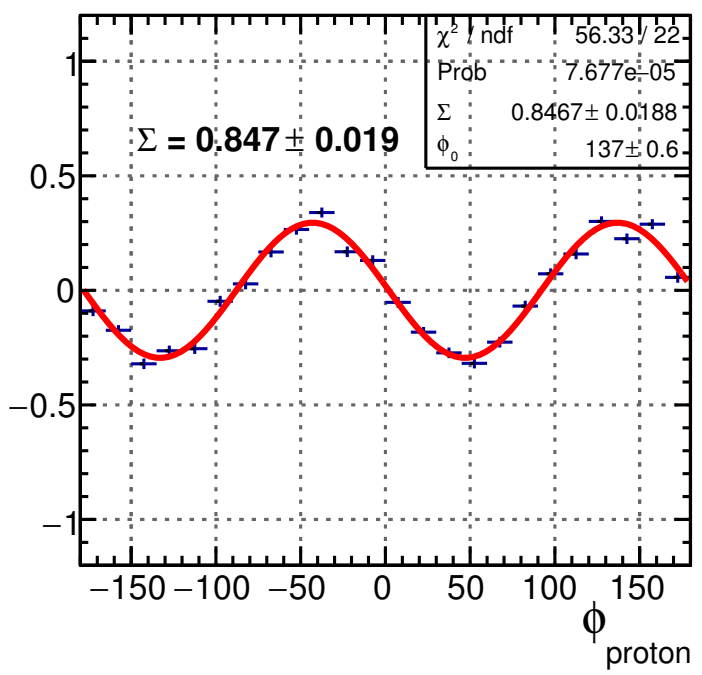

Figure 3.8: Fits to the high-statistics $t$-averaged $\eta \rightarrow 2 \gamma$ SB yield asymmetry for $0 / 90$ (left) and 45/135 (right) data sets allowing the phase offset, $\phi_{0}$, to float. The extracted phase offsets are then fixed in fits to all other yield asymmetries.

\subsubsection{Phase Offset}

The phase offset, $\phi_{0}$, can be fixed with an asymmetry fit to a high-statistics data set. Such a data set can be fit using Equation 3.7 while allowing the $\phi_{0}$ term to float. This is done for both the 0/90 and 45/135 data sets, then these phase offsets are fixed in the asymmetry fits for the $\eta$ and $\eta^{\prime}$. Since the systematics of the detector should be identical across all data from this run period, the phase shifts, which allow 
for small azimuthal misalignments between the diamond radiator and the detector, should be the same for any asymmetry analysis.

For these analyses, the phase offset is found using the $\eta \rightarrow 2 \gamma t$-averaged data. 0/90 and 45/135 data are fit, allowing $\phi_{0}$ to float. The results of these fits are shown in Figure 3.8. The extracted values, $\phi_{0} \approx 3.73^{\circ} \pm 0.61^{\circ}$ for the $0 / 90$ set and $\phi_{0} \approx 2.01^{\circ} \pm 0.65^{\circ}$ for the $45 / 135$ set, are then fixed in Equation 3.7 for all other yield asymmetries.

\subsection{Beam Asymmetry Analysis Workflow}

Combining the steps presented in this chapter, the overall workflow of the asymmetry analysis begins to take shape. After event selection, plots of the invariant mass versus $t$ and of proton $\phi$ versus $t$ (for the peak region and side-band region, if applicable) are formed, then accidental subtraction is carried out on all plots. The $\phi$ versus $t$ plots for the PARA orientation in a data set (0/90 and 45/135) are separated into bins of $t$ and scaled by $\mathrm{F}_{R}$, the flux ratio obtained from the PS data for the associated data set. The PERP orientation $\phi$ versus $t$ plots for the data sets are separated into the same $t$ bins and the yield asymmetry is formed between PARA and PERP. The resulting plots are then fit to Equation 3.7, using the fixed values of $P_{\perp}, P_{\|}$, and $\phi_{0}$ as described in Section 3.3, to extract $\Sigma_{\text {peak }}$ for $0 / 90$ and $45 / 135$ separately.

If a side-band correction must be performed, the particle invariant mass versus $t$ plot is binned in $t$ and fit to two Gaussians plus an exponential to extract $f$ for each bin. The same scaling, binning, and fits are performed on the side-band $\phi$ versus $t$

plots to extract $\Sigma_{\mathrm{SB}}$ for both the $0 / 90$ and $45 / 135$ data sets. The side-band correction is applied with Equation 3.8, giving $\Sigma_{\mathrm{COR}}$ for both data sets.

Finally, the final result is obtained by taking the weighted average of the 0/90 and $45 / 135$ results in each bin of $t$, where the weights used are the inverse-squares of the errors on the averaged values. 


\section{Chapter 4}

\section{Beam Asymmetry Results}

With the event selection and general workflow of the $\Sigma$ beam asymmetry analysis outlined in Chapter 3, the final results for the $\eta$ and $\eta^{\prime}$ can be extracted. In this chapter, specific differences between the decay channels are detailed and the invariant mass and yield distributions that are used to extract the beam asymmetries are presented. In addition, the final beam asymmetry results for the $\eta$ and $\eta^{\prime}$ obtained from this work are compared against existing results and model predictions at GlueX energies.

\section{1 $\eta$ Beam Asymmetry}

The $\eta$ beam asymmetry published by GlueX in 2017 [63] was measured using the highest statistics decay mode of the $\eta$, the decay to 2 photons. This result utilized only spring 2016 opportunistic data. With access now to the spring 2017 data, there are enough statistics to measure the beam asymmetry in the three major decay modes of the $\eta$. This allows a direct comparison to the published result using the same decay channel, plus a check on the consistency of the beam asymmetry with different decay channels to check for any systematic differences due to different particles in the final state. 
In addition, measurement of the $\eta$ channels helps with validation of the measurement of the $\eta^{\prime}$ channel. In the published GlueX $\Sigma$ result for the $\eta$, the contribution from the $\omega$ background was estimated using MC simulation of $\omega \rightarrow \pi^{0} \gamma$ events. Those events that pass the event selection for the $\eta \rightarrow 2 \gamma$ channel were used to predict the line shape of the $\omega$ background under the $\eta$ peak. In this way, the dilution factor, $f$, was obtained, and a systematic error on the $\eta$ beam asymmetry was assigned due to the determination of the contribution of the $\omega$ background. In this thesis, the method for extracting this contribution is different. The motivation for using a sideband region to estimate the background asymmetry is that the background of the $\eta^{\prime}$ channel is not dominantly from a single source like the $\eta$ background. Thus, a more encompassing method was necessary. Comparing results in all $\eta$ decay channels to one another and to past results helps validate the side-band asymmetry correction method used for the $\eta^{\prime}$ asymmetry analysis.

Note also that no systematic uncertainty studies are conducted for the three $\eta$ analyses. The reasons behind this decision are discussed briefly in Section 5.2.

\subsection{1 $\eta \rightarrow \pi^{+} \pi^{-} \pi^{0}$}

Figure 4.1 shows the $\pi^{+} \pi^{-} \pi^{0}$ mass spectra for four regions of $t$. The majority of the events are in the two bins with $|-t|<0.5$, and the fractional background under the peak is less than $1 \%$ in the highest $-t$ bin. Even so, the background is non-negligible, so a side-band correction must be performed. Each plot features a fit to Equation 3.4. An exponential can be used to model the background because it is almost entirely caused by the exponentially decaying tail of the $\omega$ with little to no other contribution. The larger of the two Gaussian widths from the $t$-averaged fit (shown previously in Figure 3.7 ) defines the $\pm 2 \sigma$ mass window for the peak region, shown with blue delimiting lines in each plot. Since the background distribution is almost entirely composed of $\omega$ events, the asymmetry for events in the mass side-band 
is expected to be mass-independent (a notion that is tested further in Section 5.2.1.1) and equal to the asymmetry of the background under the peak. As such, the sideband mass range is chosen from $0.63 \mathrm{GeV}$ to $0.80 \mathrm{GeV}$ to maximize the statistics available for the side-band asymmetry correction. The dilution factor, $f$, is displayed on each spectrum, along with the side-band region used, shown with purple delimiting lines.
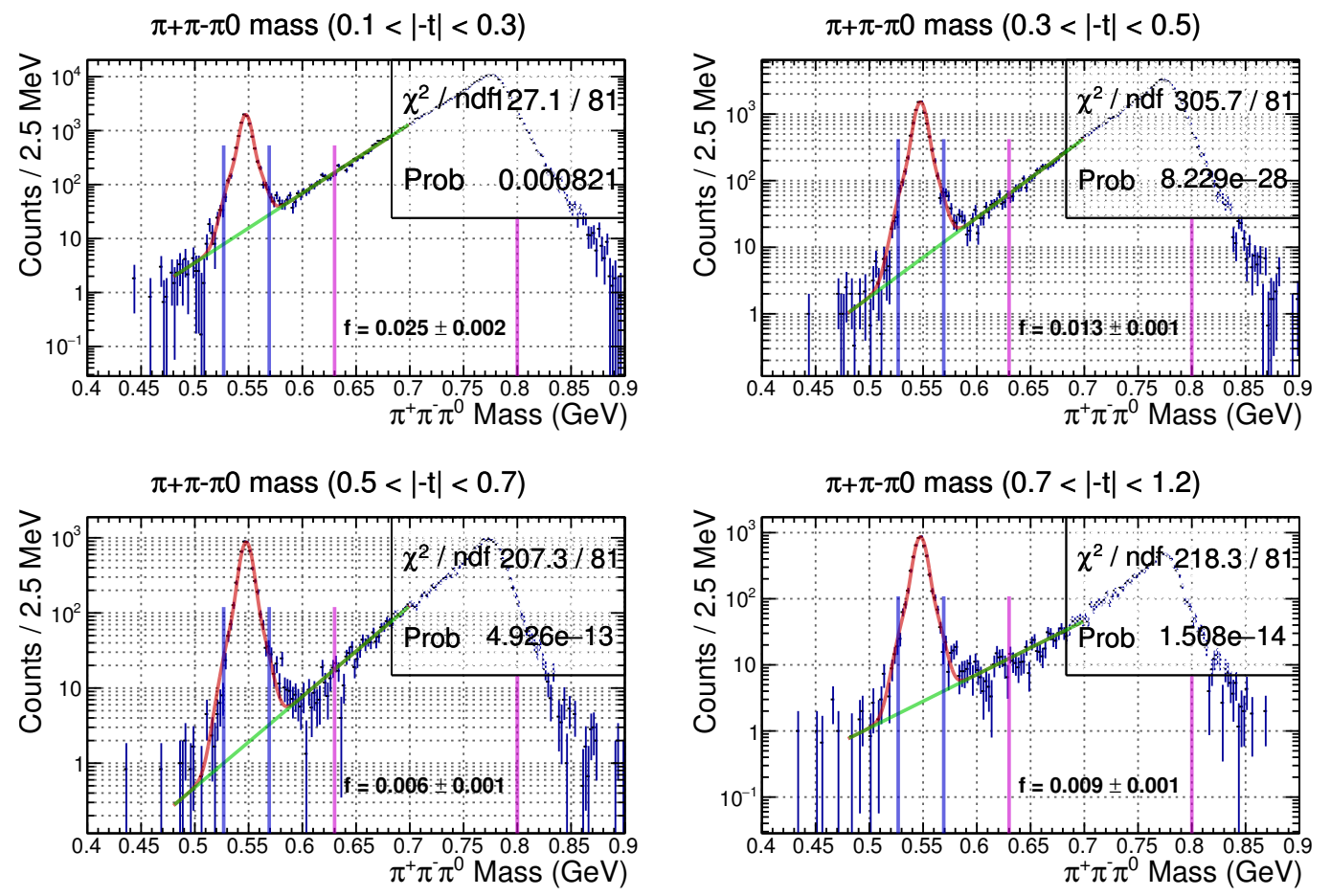

Figure 4.1: $\pi^{+} \pi^{-} \pi^{0}$ mass spectra and dilution factors for four ranges of $t$ (all polarization orientations). The blue delimiting lines denote the peak region, and the purple delimiting lines denote the side-band region (colour online).

Figure 4.2 shows the accidental-subtracted yield asymmetry $\phi$ distributions for the $\eta$ peak region (0/90 data set). Similar to the mass spectra, the panels are for four ranges of $t$. The distributions are fit to Equation 3.7 and the resulting $\Sigma_{\text {peak }}$ values are displayed on the plots. In all $t$ bins, the asymmetry is highly positive at around 0.8 or 0.9 , implying dominantly natural parity exchange during $\eta$ photoproduction.

Figure 4.3 shows the accidental-subtracted yield asymmetry $\phi$ distributions for 

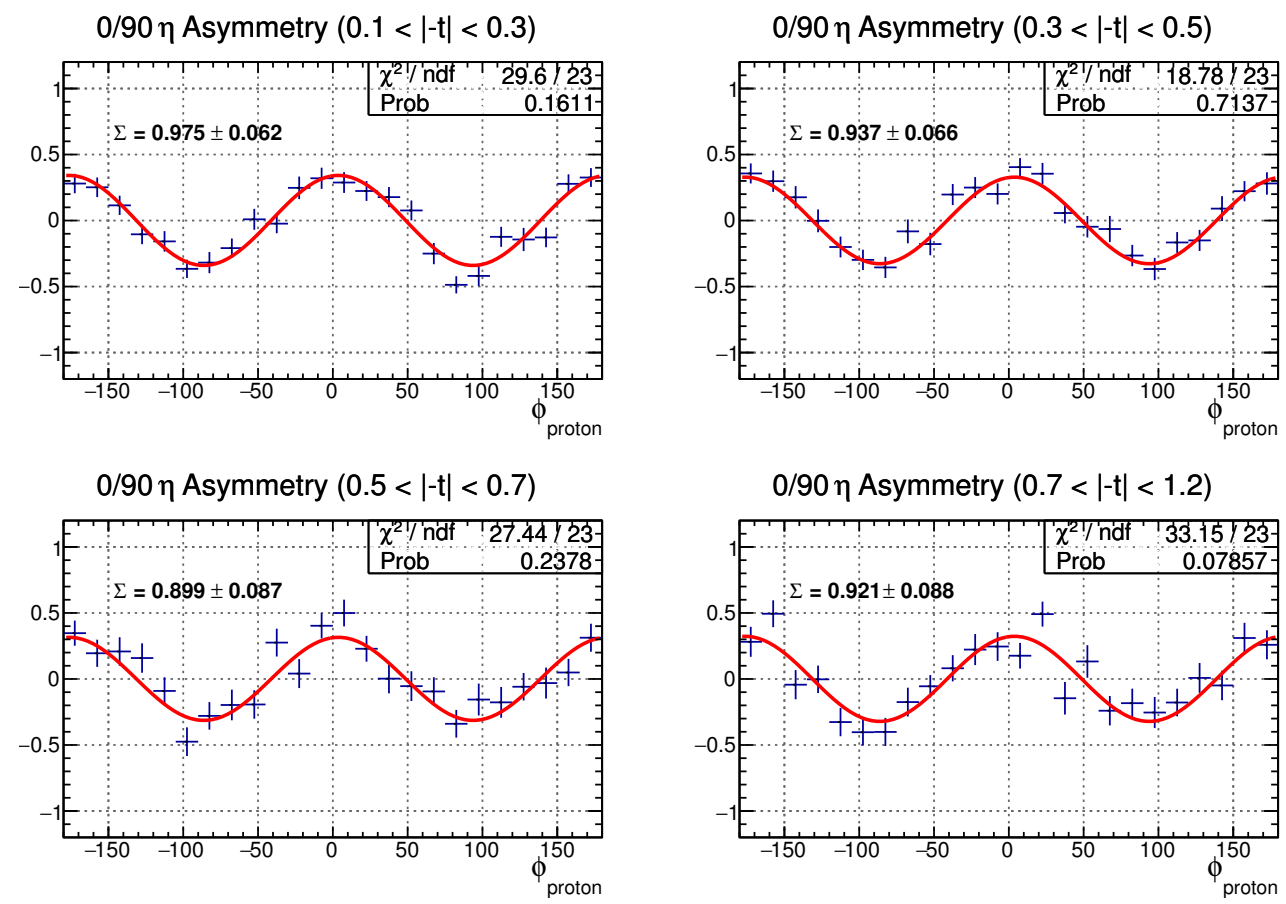

Figure 4.2: $\quad \eta \rightarrow \pi^{+} \pi^{-} \pi^{0} 0 / 90$ yield asymmetry distributions in the $\eta$ peak region for four ranges of $t$.
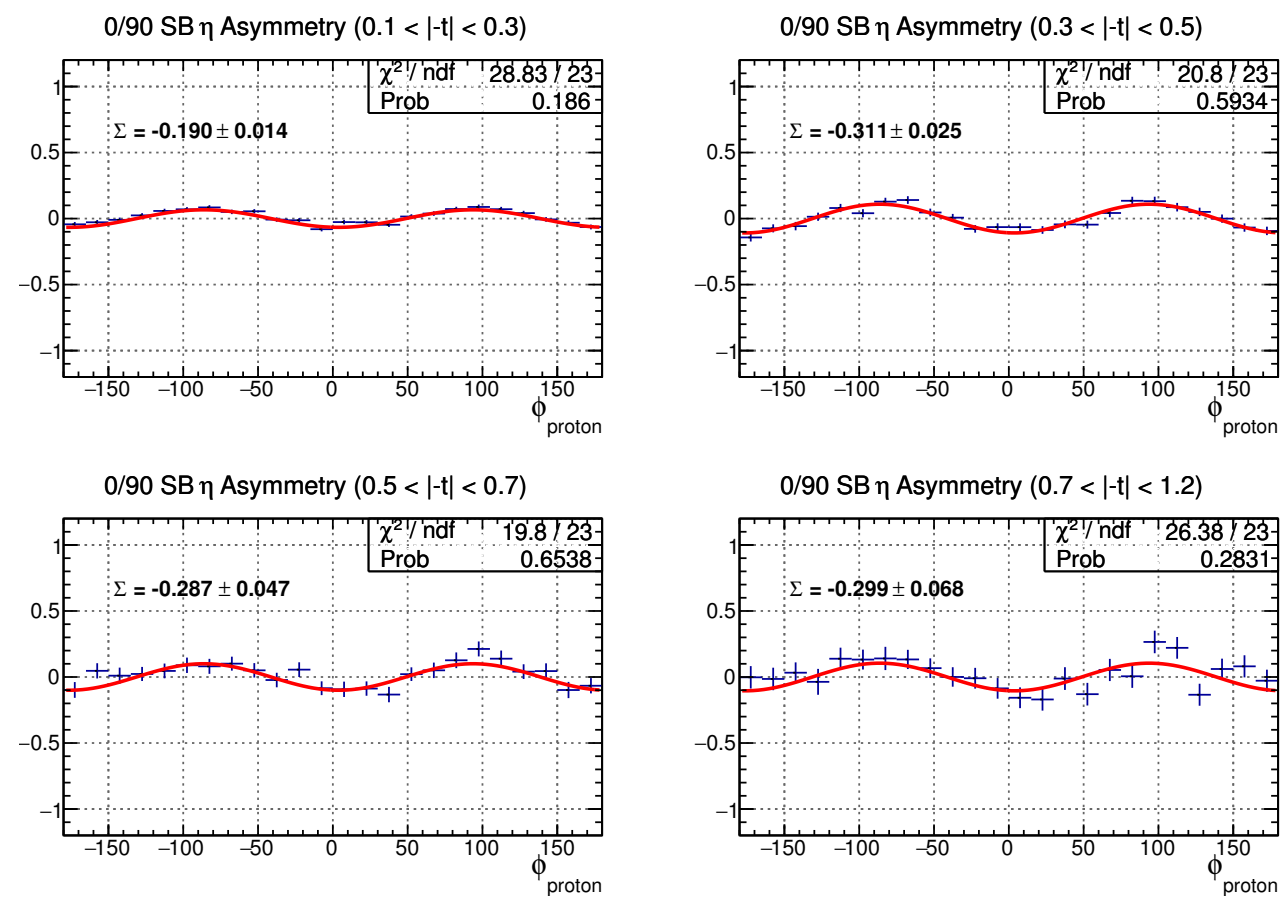

Figure 4.3: $\quad \eta \rightarrow \pi^{+} \pi^{-} \pi^{0} 0 / 90$ yield asymmetry distributions in the side-band region for four ranges of $t$. 
the side-band region in the same $t$ ranges (0/90 data set). Here, the asymmetries are negative, between -0.1 and -0.4 . Because the asymmetry as found in the peak region and the side-band region are rather different in magnitude, large amounts of background under the $\eta$ peak could strongly affect the extracted beam asymmetry. Since the SB asymmetries are less positive than the peak asymmetries, the corrected asymmetries, found with Equation 3.8, are more positive than the peak asymmetries. However, since the dilution factors in this channel are small (no more than 2.5\%), the corrections are also small.
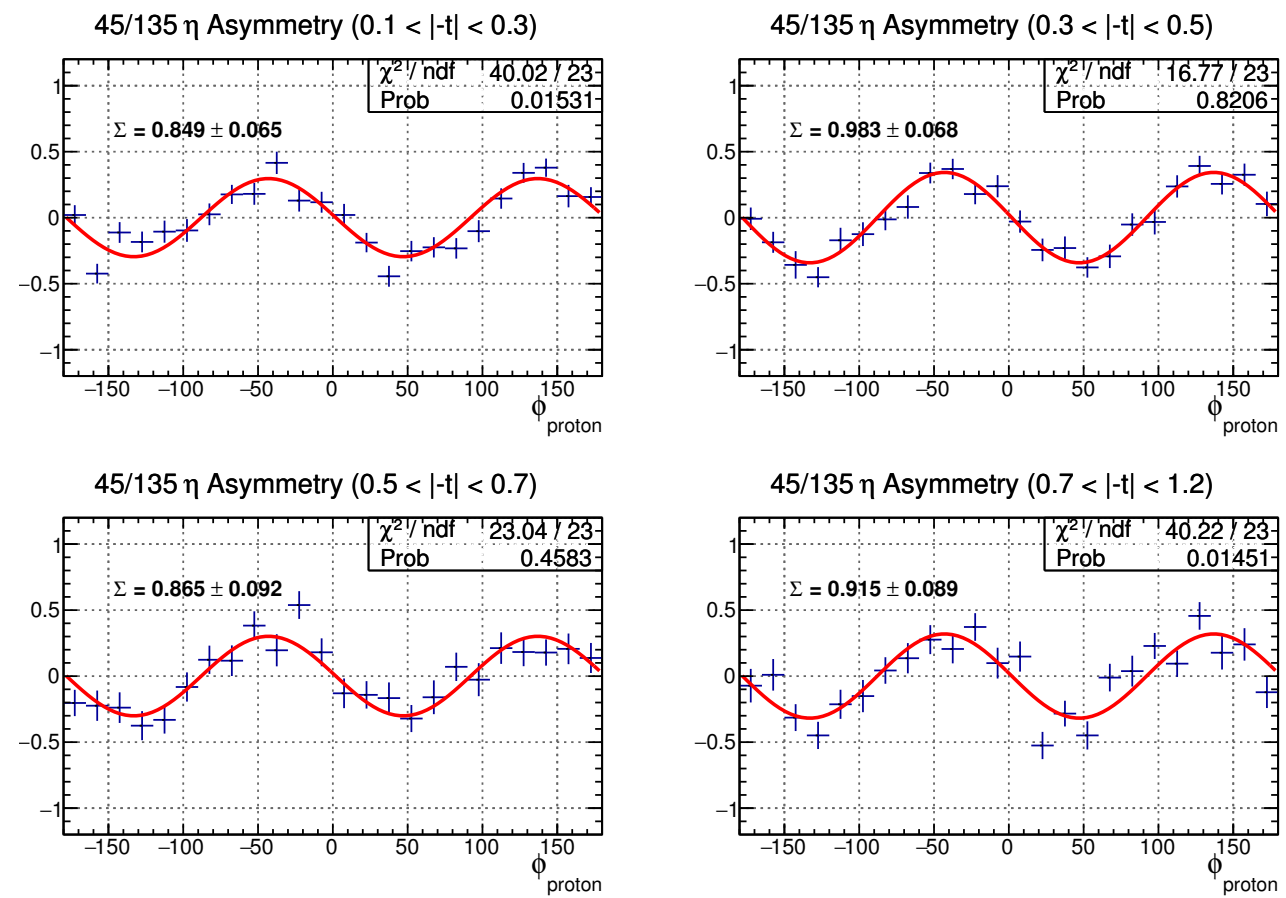

Figure 4.4: $\eta \rightarrow \pi^{+} \pi^{-} \pi^{0} 45 / 135$ yield asymmetry distributions in the $\eta$ peak region for four ranges of $t$.

The similar plots for the 45/135 data set are shown in Figures 4.4 and 4.5, and Figure 4.6 summarizes $\Sigma_{\text {peak }}, \Sigma_{S B}$, and $\Sigma_{C O R}$ versus $t$ for both the $0 / 90$ and $45 / 135$ data sets. The vertical error bars here are statistical only. Throughout this thesis, a horizontal error bar on an asymmetry data point represents only the size of the associated $t$ bin. 

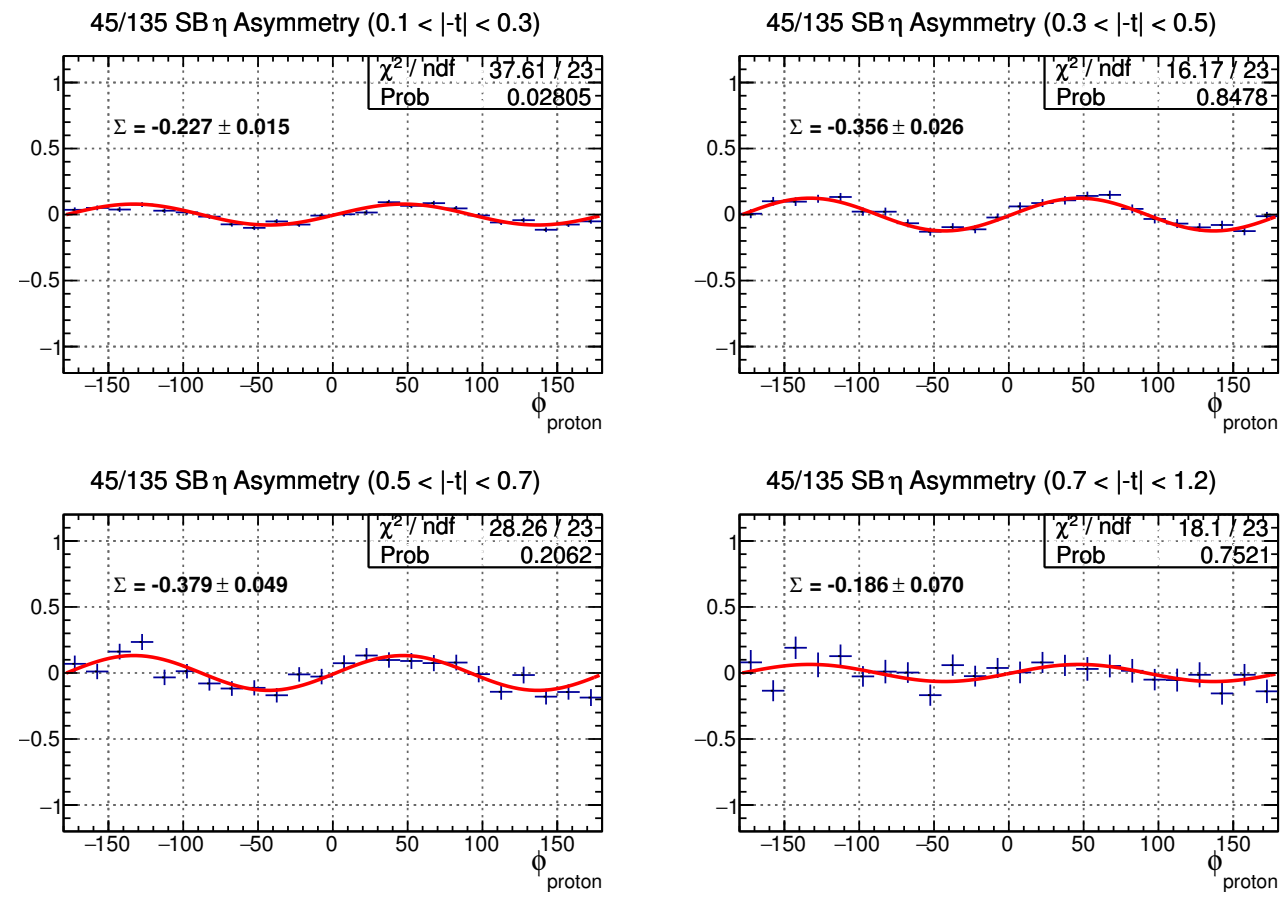

Figure 4.5: $\quad \eta \rightarrow \pi^{+} \pi^{-} \pi^{0} \quad 45 / 135$ yield asymmetry distributions in the side-band region for four ranges of $t$.

Peak $\eta \Sigma$ vs -t

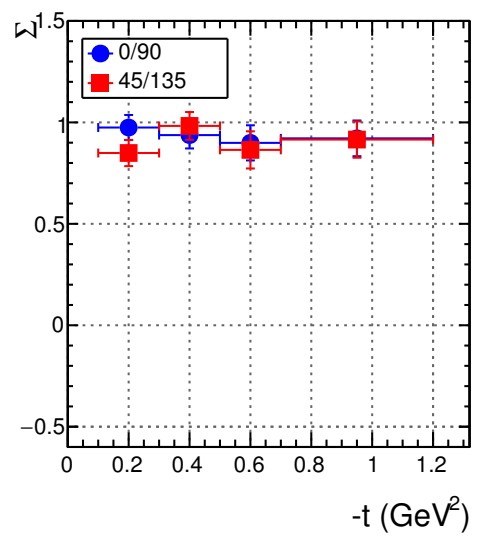

SB $\eta \Sigma$ vs -t

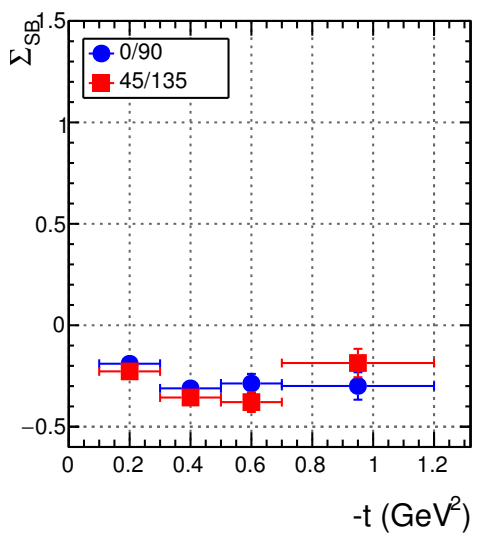

SB-Corrected $\eta \Sigma$ vs -t

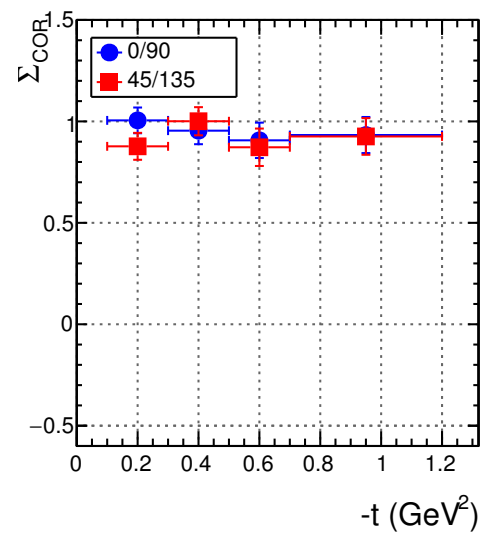

Figure 4.6: $\eta$ beam asymmetries versus $t$ for the $\eta \rightarrow \pi^{+} \pi^{-} \pi^{0}$ decay channel, from left to right: $\Sigma_{\text {peak }}, \Sigma_{\mathrm{SB}}$, and $\Sigma_{\mathrm{COR}}$. 0/90 data are in blue (circles), 45/135 data are in red (squares). Errors are statistical only (colour online).

In order to quantify the similarity of the $0 / 90$ and 45/135 results before averaging them, a $\chi^{2}$ test is performed. The $\chi^{2}$ test metric for comparison of two weighted data sets, in terms of the asymmetries and statistical errors on the asymmetries in each 
bin of $t$, is given by Equation 4.1 [110].

$$
\begin{aligned}
& \chi^{2}=\sum_{i=1}^{n} \frac{\left(W_{45 / 135} \Sigma_{i}^{0 / 90}-W_{0 / 90} \Sigma_{i}^{45 / 135}\right)^{2}}{W_{0 / 90}^{2} \delta_{\Sigma_{i}^{4 / 135}}^{2}+W_{45 / 135}^{2} \delta_{\Sigma_{i}^{0 / 90}}^{2}} \\
& \text { with } \quad W_{0 / 90}=\sum_{i=1}^{n} \Sigma_{i}^{0 / 90} \text { and } W_{45 / 135}=\sum_{i=1}^{n} \Sigma_{i}^{45 / 135}
\end{aligned}
$$

Here, $n$ is the number of bins in the distributions (for comparison of asymmetry results, $n=4$, the number of bins of $t$ used $), \Sigma_{i}^{0 / 90}\left(\Sigma_{i}^{45 / 135}\right)$ is the beam asymmetry (SB-corrected, if applicable) in the $i$ th bin of $t$ for the $0 / 90(45 / 135)$ data set, and $\delta_{\Sigma_{i}^{0 / 90}}^{2}\left(\delta_{\Sigma_{i}^{45 / 135}}^{2}\right)$ is the error on that asymmetry. In essence, this test statistic quantifies the difference between the 0/90 and 45/135 data sets by comparing their asymmetries in each bin of $t$.

Generally, such test metrics follow the ' $\chi^{2}$ distribution' when dealing with independent, standard normal, random variables. The $\chi^{2}$ distribution's probability density function (PDF) is given by Equation 4.2 and is shown graphically for a variety of degrees of freedom, $n d f=n-1$, in Figure 4.7 .

$$
f_{n d f}(x)=\frac{x^{\frac{n d f}{2}}-1 e^{-\frac{x}{2}}}{2^{\frac{n d f}{2}} \Gamma\left(\frac{n d f}{2}\right)}, \quad x>0
$$

As a test of whether the 0/90 and 45/135 asymmetries are significantly different, this $\chi^{2}$ test statistic is calculated, and the $p$-value of the test statistic is computed as $p=\int_{\chi^{2}}^{\infty} f_{n d f}(x)$. Since 4 bins of $t$ are used in the asymmetry analyses, $n d f=3$. The $p$-value corresponds to the probability of observing a $\chi^{2}$ at least as large as the test statistic in a $\chi^{2}$ distribution. If the test statistic is large, the $p$-value will be small, meaning that the two distributions are more likely to be quantitatively different. A $p$ value smaller than a chosen significance level is sufficient to reject the null hypothesis of the test, which in this case is the hypothesis that the 0/90 and 45/135 asymmetries represent identical distributions, at that significance level. The chosen significance 


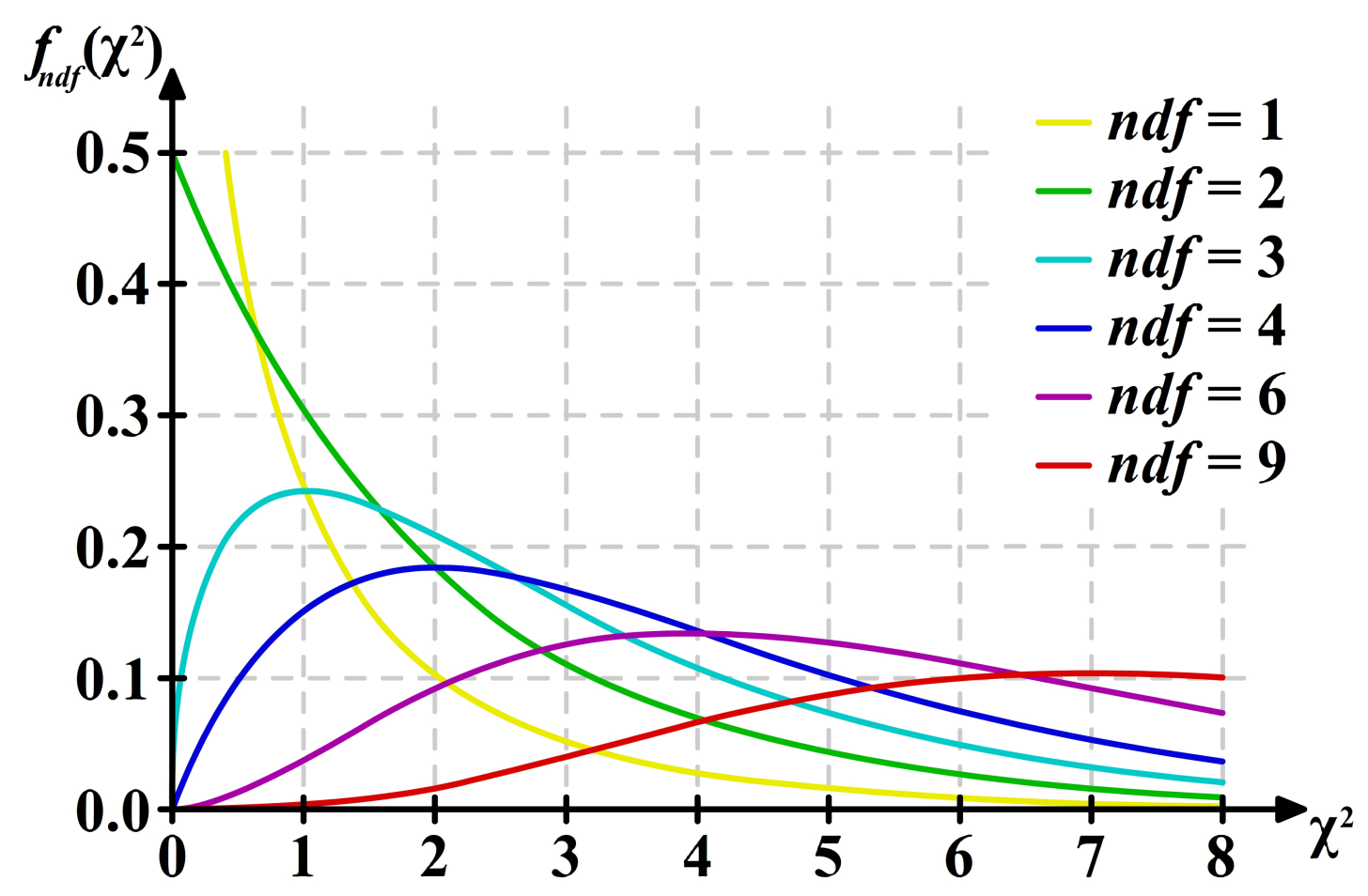

Figure 4.7: $\chi^{2}$ PDF for various degrees of freedom, $n d f$.

level is commonly taken to be 0.05 , meaning the test statistic must be extreme enough that there is less than a $5 \%$ chance to pull a more extreme test statistic from a $\chi^{2}$ distribution to claim that the two tested distributions are significantly different from each other.

For the $\eta \rightarrow \pi^{+} \pi^{-} \pi^{0} 0 / 90$ and $45 / 135$ asymmetry results, the $p$-value is 0.611 . Because this is much larger than 0.05 , the hypothesis that the $0 / 90$ and $45 / 135$ results represent an identical distribution can be accepted, and the results of the two data sets can be averaged. Thus, the final result is calculated as the weighted average between the 0/90 and 45/135 corrected asymmetries, as in Equation 4.3.

$$
\begin{aligned}
\Sigma_{\mathrm{AVG}} & =\frac{\Sigma_{0 / 90}\left(\delta_{\Sigma_{0 / 90}}\right)^{-2}+\Sigma_{45 / 135}\left(\delta_{\Sigma_{45 / 135}}\right)^{-2}}{\left(\delta_{\Sigma_{0 / 90}}\right)^{-2}+\left(\delta_{\Sigma_{45 / 135}}\right)^{-2}} \\
\delta_{\Sigma_{\mathrm{AVG}}} & =\frac{1}{\sqrt{\left(\delta_{\Sigma_{0 / 90}}\right)^{-2}+\left(\delta_{\Sigma_{45 / 135}}\right)^{-2}}}
\end{aligned}
$$


The result is shown in Figure 4.8, and is tabulated in Table 4.1. Again, the vertical error bars are statistical only. This figure (and subsequent similar results plots) also features the normalization uncertainty, discussed in Section 3.3.2, which represents the uncertainty in the overall scale of the averaged beam asymmetry results due to statistical and systematic errors on the polarization measurements from the TPOL. $\Sigma$ as found in this decay channel appears flat versus $t$.

\section{$\eta \Sigma$ vs -t}

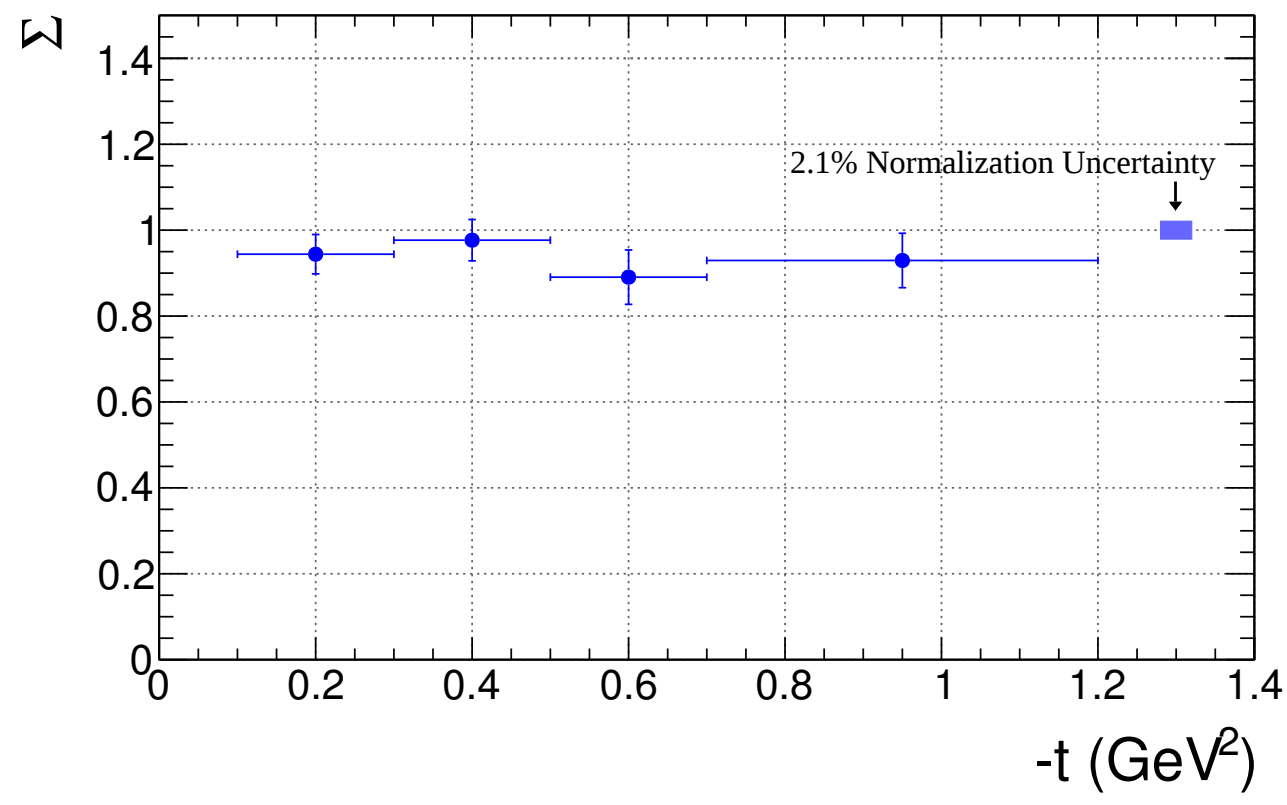

Figure 4.8: Weighted average of the 0/90 and 45/135 side-band corrected beam asymmetries for the $\eta \rightarrow \pi^{+} \pi^{-} \pi^{0}$ decay channel. Vertical error bars are statistical only, and the height of the marked box represents the $2.1 \%$ normalization uncertainty.

\begin{tabular}{|c||c|c|}
\hline$t$ Range $\left(\mathrm{GeV}^{2}\right)$ & $\Sigma_{\mathrm{AVG}}$ & $\delta_{\Sigma_{\mathrm{AVG}}}$ \\
\hline \hline $0.1<-t<0.3$ & 0.944 & 0.046 \\
\hline $0.3<-t<0.5$ & 0.977 & 0.048 \\
\hline $0.5<-t<0.7$ & 0.891 & 0.063 \\
\hline $0.7<-t<1.2$ & 0.929 & 0.063 \\
\hline
\end{tabular}

Table 4.1: Average asymmetries and statistical errors for the $\eta \rightarrow \pi^{+} \pi^{-} \pi^{0}$ decay channel. 

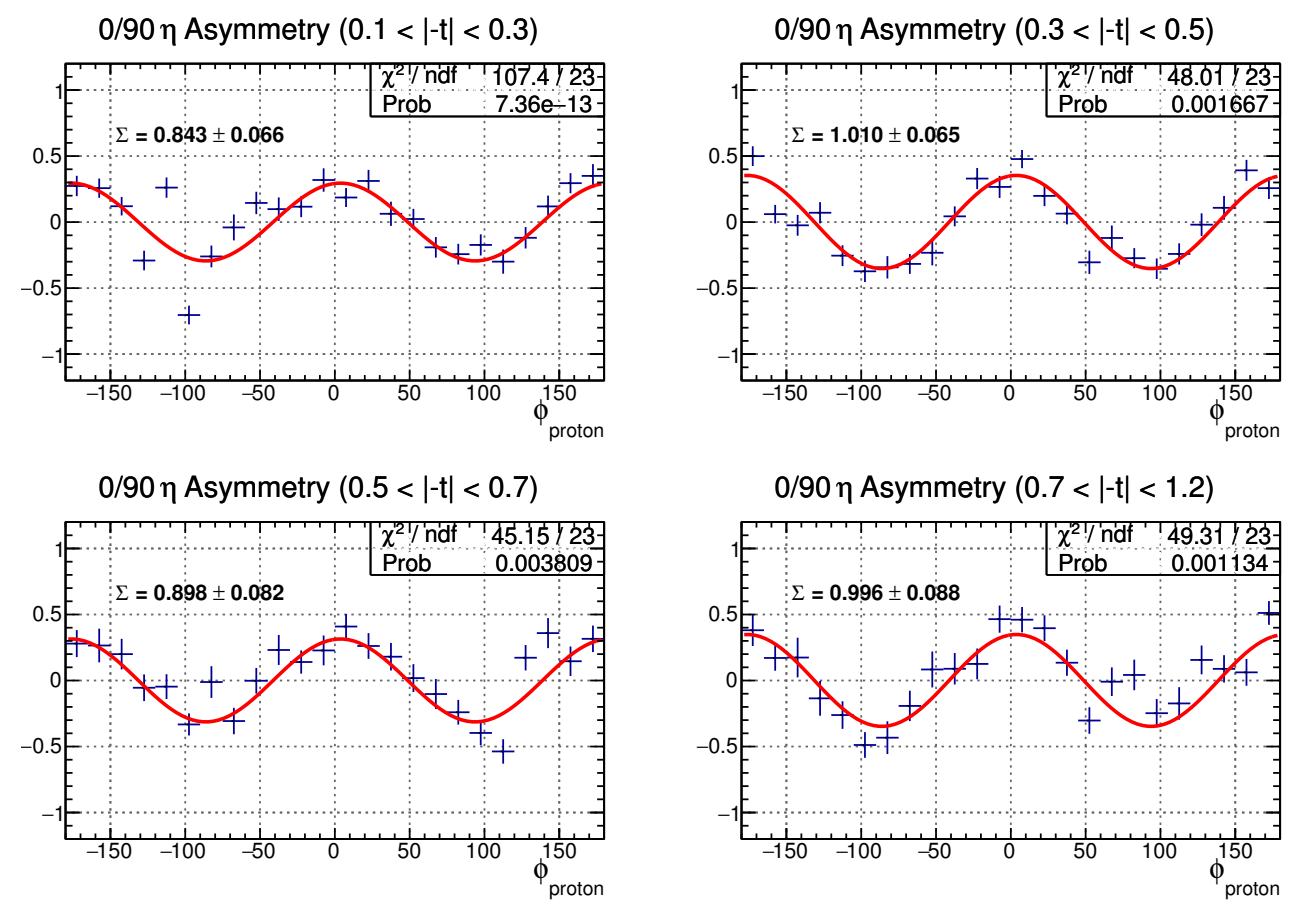

Figure 4.9: $\quad \eta \rightarrow 3 \pi^{0} 0 / 90$ yield asymmetry distributions in the $\eta$ peak region for four ranges of $t$.
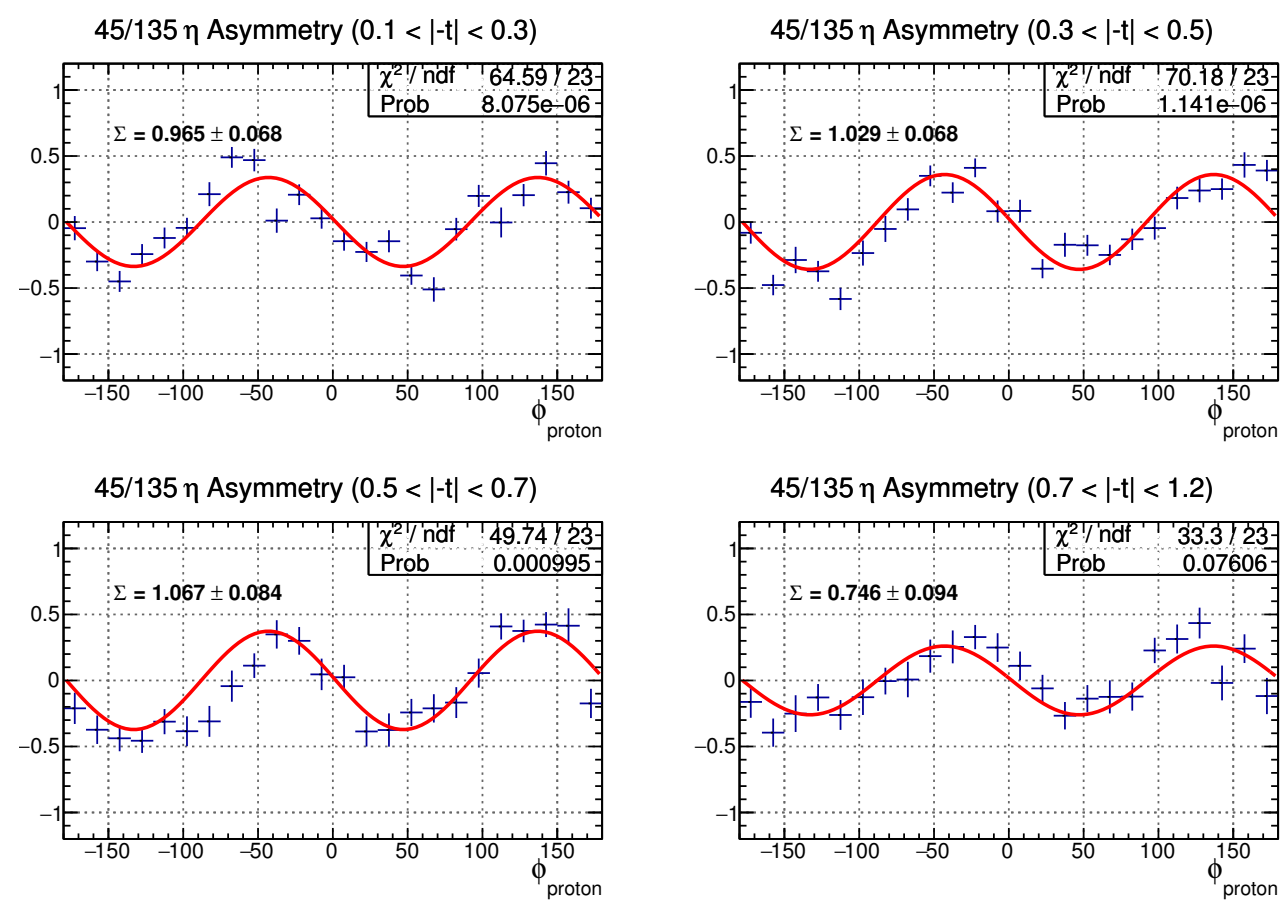

Figure 4.10: $\quad \eta \rightarrow 3 \pi^{0} 45 / 135$ yield asymmetry distributions in the $\eta$ peak region for four ranges of $t$. 


\subsection{2 $\quad \eta \rightarrow 3 \pi^{0}$}

The $t$-averaged $3 \pi^{0}$ mass spectrum was shown previously in Figure 3.7. The background in this channel is practically non-existent, so the fit consists only of a double Gaussian, and no side-band correction is necessary. The $2 \sigma$ peak region shown in the plot as blue delimiting lines is based on the larger of the two Gaussian widths from the fit.

Figures 4.9 and 4.10 show the 0/90 and 45/135 accidental-subtracted yield asymmetry $\phi$ distributions (binned in $t$ ) for $\eta$ peak region events respectively, and Figure 4.11 shows $\Sigma_{\text {peak }}$ versus $t$ for both the $0 / 90$ and $45 / 135$ data sets. The $p$-value for the $\eta \rightarrow 3 \pi^{0} 0 / 90$ and $45 / 135$ asymmetries is 0.066 . For a 0.05 significance level, the null hypothesis is accepted and the results are averaged. This relatively small $p$-value likely stems from the fact that some data in the highest $-t$ bins of the $45 / 135$ data set (see Figure 4.10) are not well-described by the corresponding fits.

\section{Peak $\eta \Sigma$ vs -t}

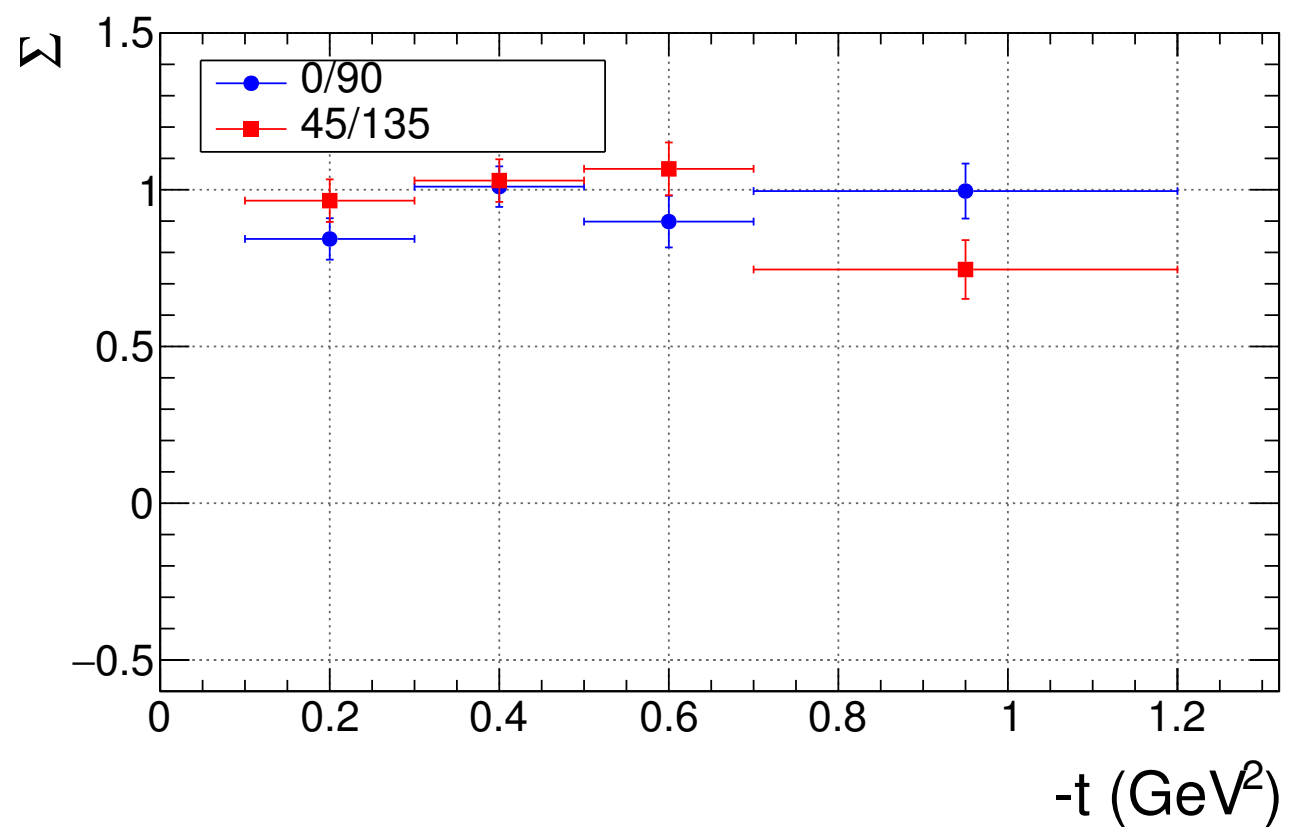

Figure 4.11: $\eta$ beam asymmetries $\left(\Sigma_{\text {peak }}\right)$ versus $t$ for the $\eta \rightarrow 3 \pi^{0}$ decay channel. 0/90 data are in blue (circles), 45/135 data are in red (squares). Errors are statistical only (colour online). 
Figure 4.12 shows the weighted average of the $0 / 90$ and $45 / 135$ results, and the same results are tabulated in Table 4.2. The vertical error bars are statistical only. The asymmetry in this decay channel shows more structure than in the 3piq channel, though the error bars on each point are still quite large due to relatively low statistics.

\section{$\eta \Sigma$ vs -t}

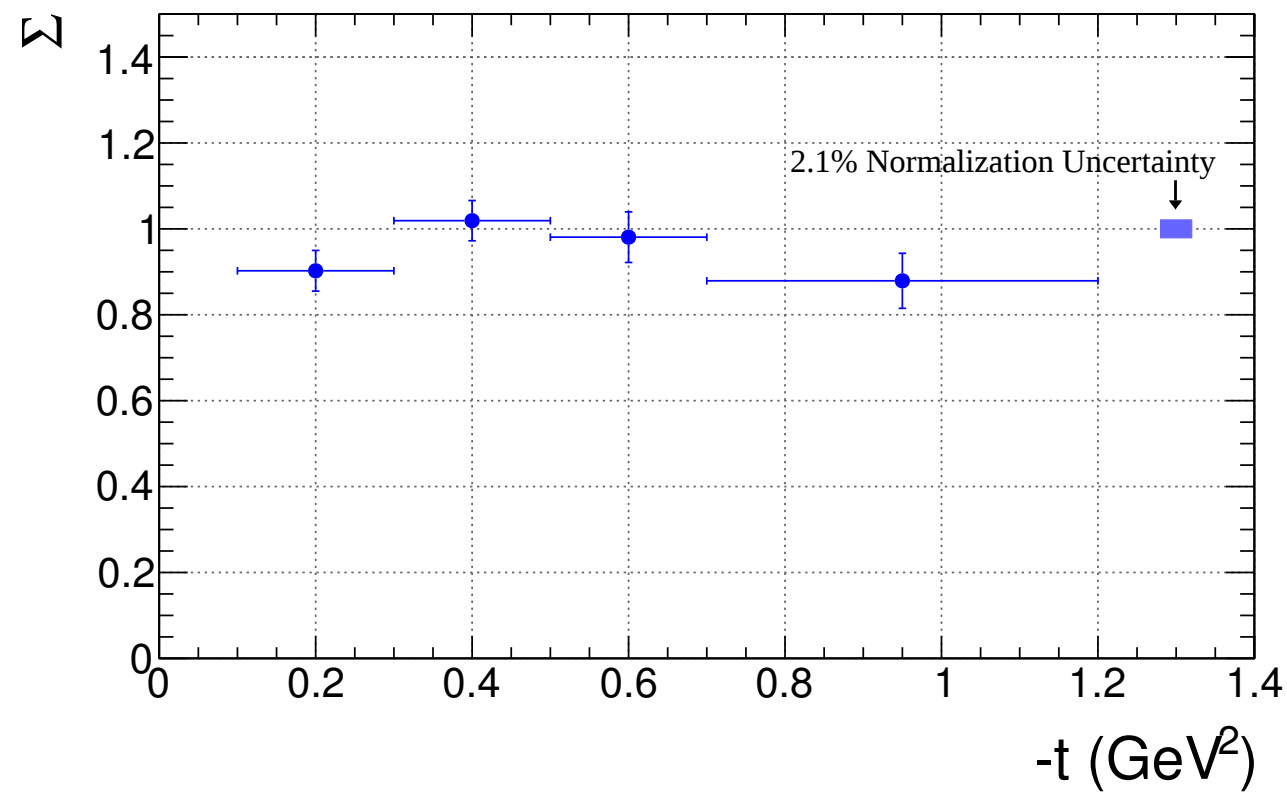

Figure 4.12: Weighted average of the 0/90 and 45/135 beam asymmetries for the $\eta \rightarrow 3 \pi^{0}$ decay channel. Vertical error bars are statistical only, and the height of the marked box represents the $2.1 \%$ normalization uncertainty.

\begin{tabular}{|c||c|c|}
\hline$t$ Range $\left(\mathrm{GeV}^{2}\right)$ & $\Sigma_{\mathrm{AVG}}$ & $\delta_{\Sigma_{\mathrm{AVG}}}$ \\
\hline \hline $0.1<-t<0.3$ & 0.902 & 0.047 \\
\hline $0.3<-t<0.5$ & 1.019 & 0.047 \\
\hline $0.5<-t<0.7$ & 0.981 & 0.059 \\
\hline $0.7<-t<1.2$ & 0.879 & 0.064 \\
\hline
\end{tabular}

Table 4.2: Average asymmetries and statistical errors for the $\eta \rightarrow 3 \pi^{0}$ decay channel.

\subsection{3 $\quad \eta \rightarrow 2 \gamma$}

Figure 4.13 shows the $2 \gamma$ mass spectra (binned in $t$ ). This channel features approximately a factor of 6 times more statistics than the 3piq decay channel, but this channel also has significant background $(12.1 \%$ at low $-t$, and still $4 \%$ in the highest 

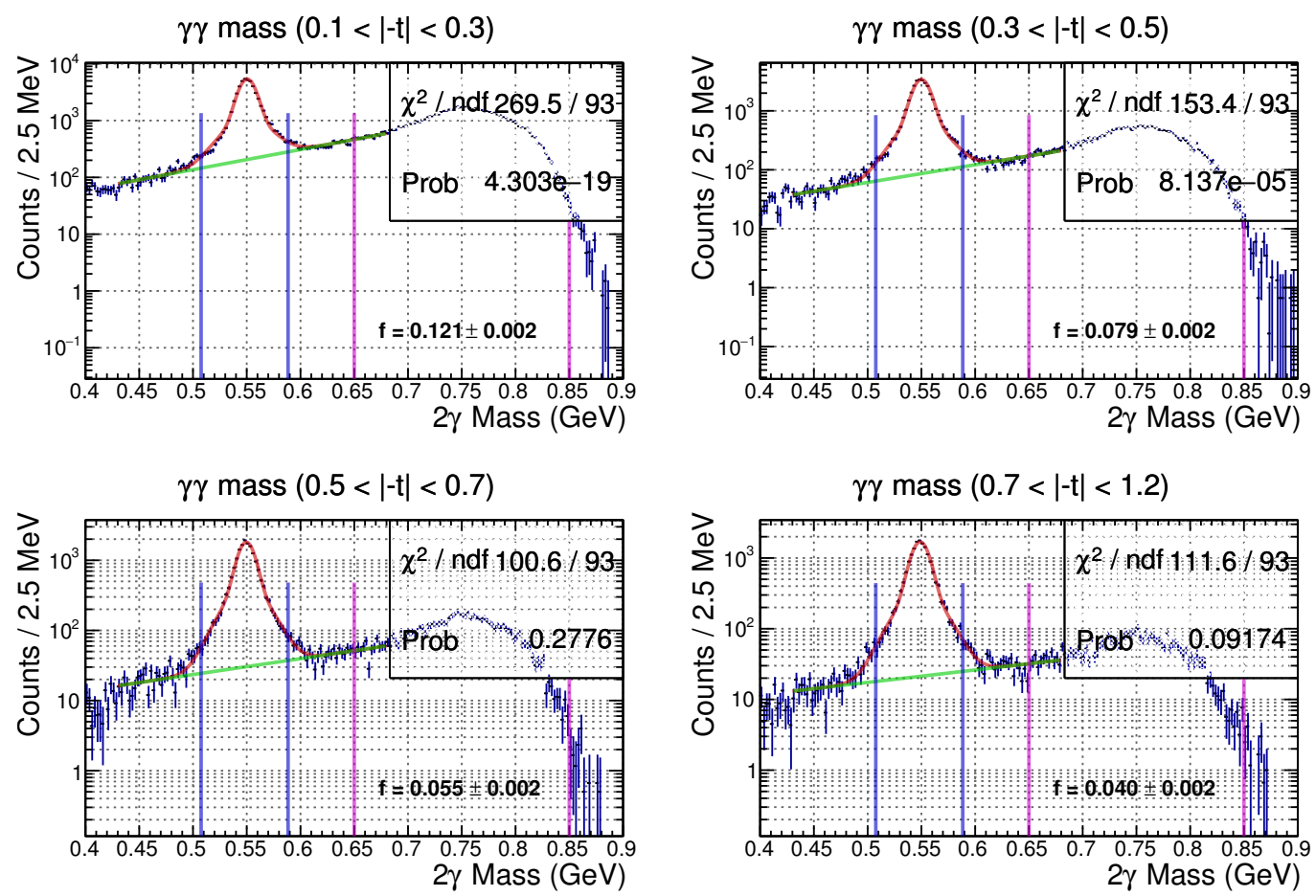

Figure 4.13: $2 \gamma$ mass spectra, from the decay of the $\eta$, and dilution factors for four ranges of $t$ (all polarization orientations). The blue delimiting lines denote the peak region, and the purple delimiting lines denote the side-band region (colour online).

$-t$ bin). The spectra are fit to Equation 3.4 and side-band corrections are done. The background is modelled by an exponential since it is almost entirely due to $\omega$ 'leakage' ( $\omega \rightarrow \pi^{0} \gamma$ events with a missing photon). For the same reasons as the 3piq decay channel, the side-band region is chosen to maximize statistics by including the majority of the $\omega$ leakage spectrum (masses between $0.65 \mathrm{GeV}$ and $0.85 \mathrm{GeV}$ ), under the assumption that the side-band asymmetry is mass-independent and equal to the asymmetry of background events under the peak. As previously mentioned, this assumption is tested in Section 5.2.1.1. Extracted dilution factors are displayed on the plots, along with the peak region (defined by the larger of the Gaussian widths from the $t$-averaged fit, shown previously in Figure 3.7) and the side-band region.

Figures 4.14 and 4.15 show the accidental-subtracted yield asymmetry $\phi$ distributions for $\eta$ peak region and side-band region events (0/90 data set). 

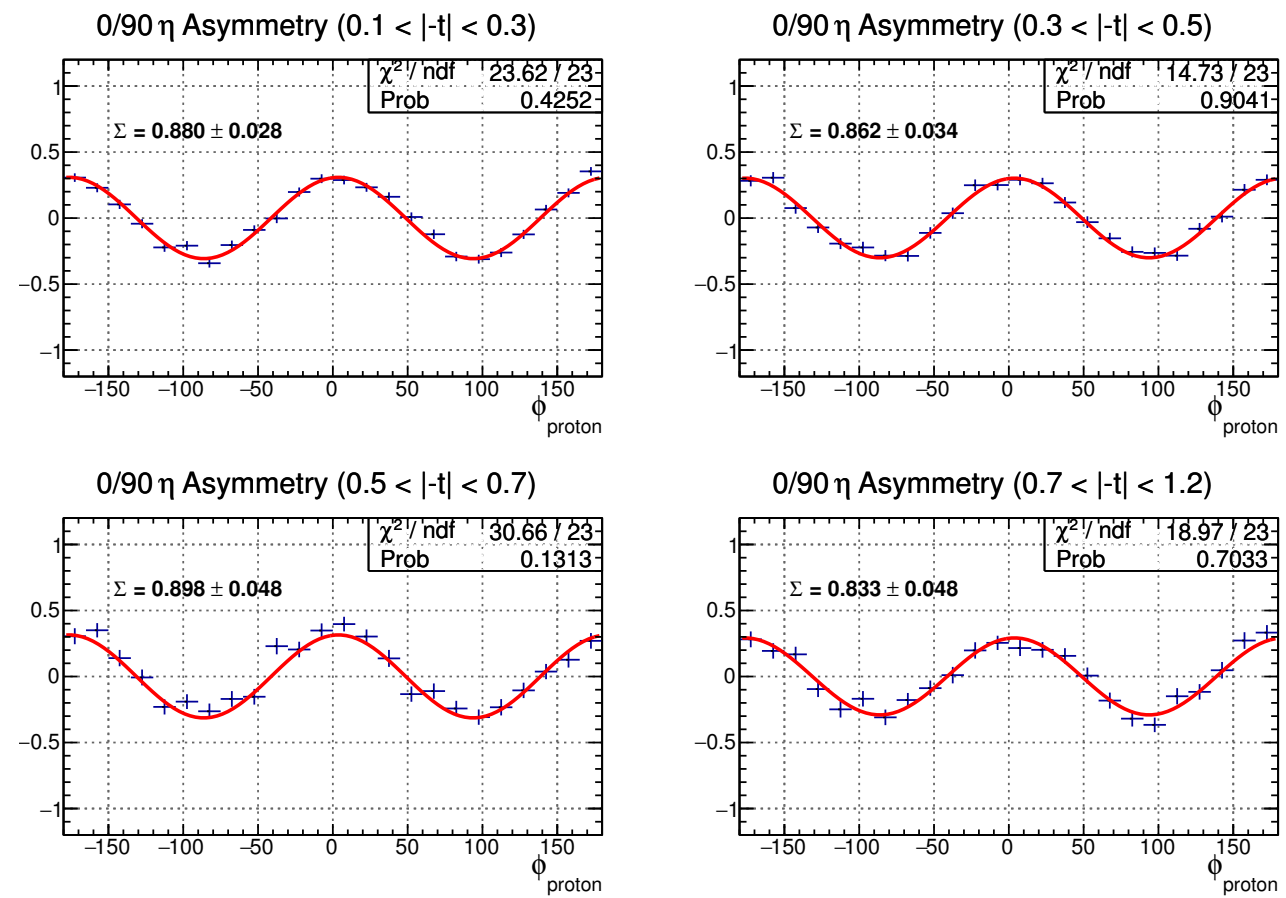

Figure 4.14: $\eta \rightarrow 2 \gamma$ 0/90 yield asymmetry distributions in the $\eta$ peak region for four ranges of $t$.
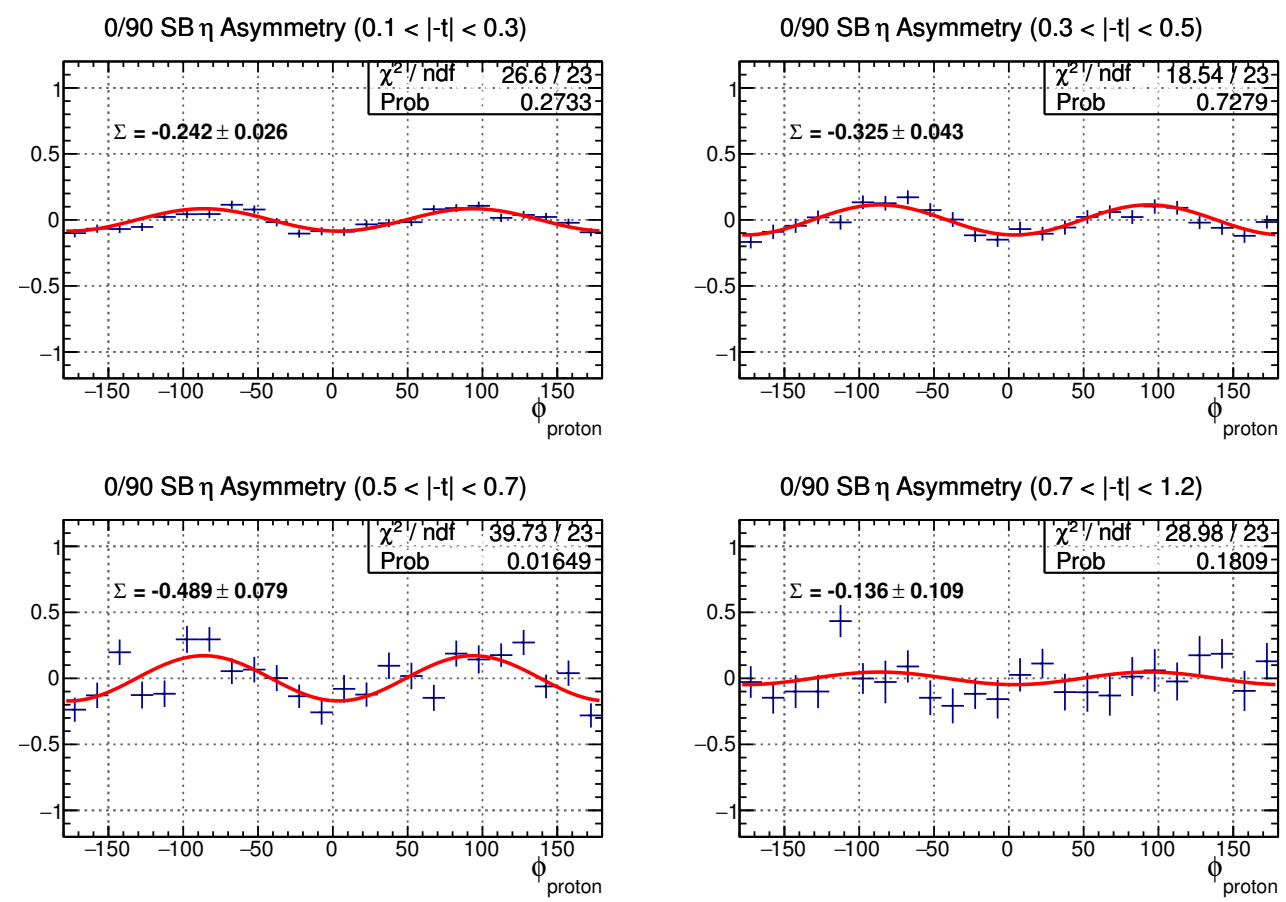

Figure 4.15: $\quad \eta \rightarrow 2 \gamma 0 / 90$ yield asymmetry distributions in the side-band region for four ranges of $t$. 

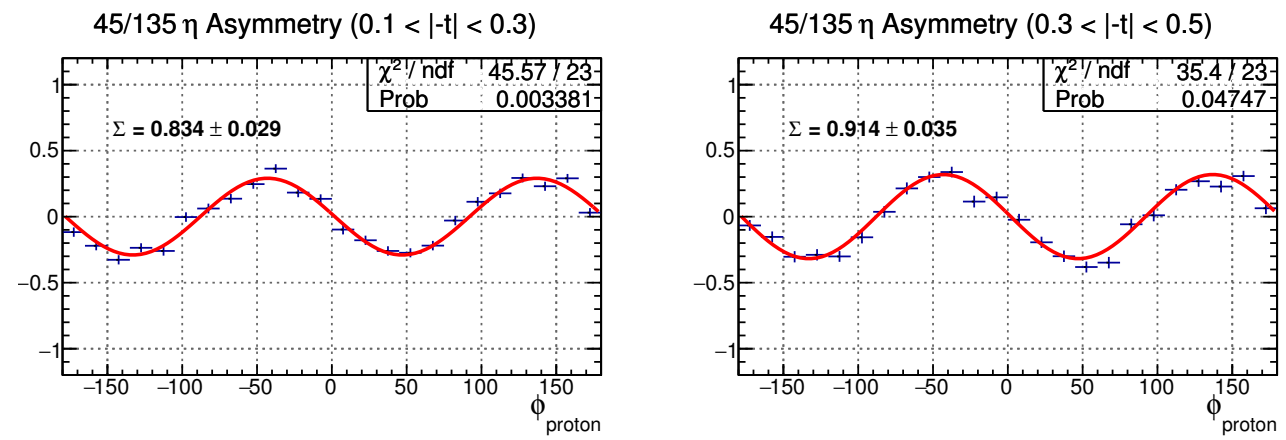

45/135 $\eta$ Asymmetry $(0.5<|-t|<0.7)$
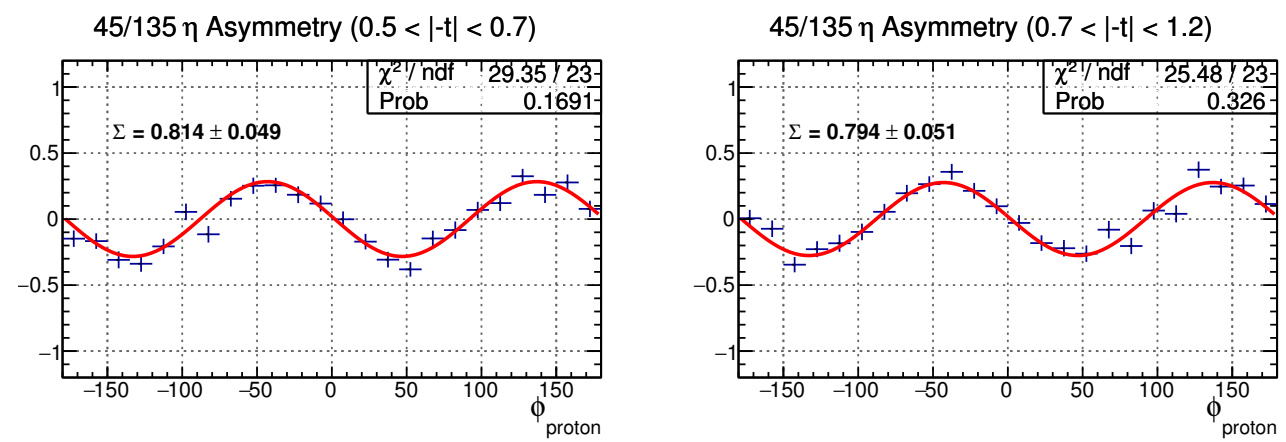

Figure 4.16: $\quad \eta \rightarrow 2 \gamma$ 45/135 yield asymmetry distributions in the $\eta$ peak region for four ranges of $t$.
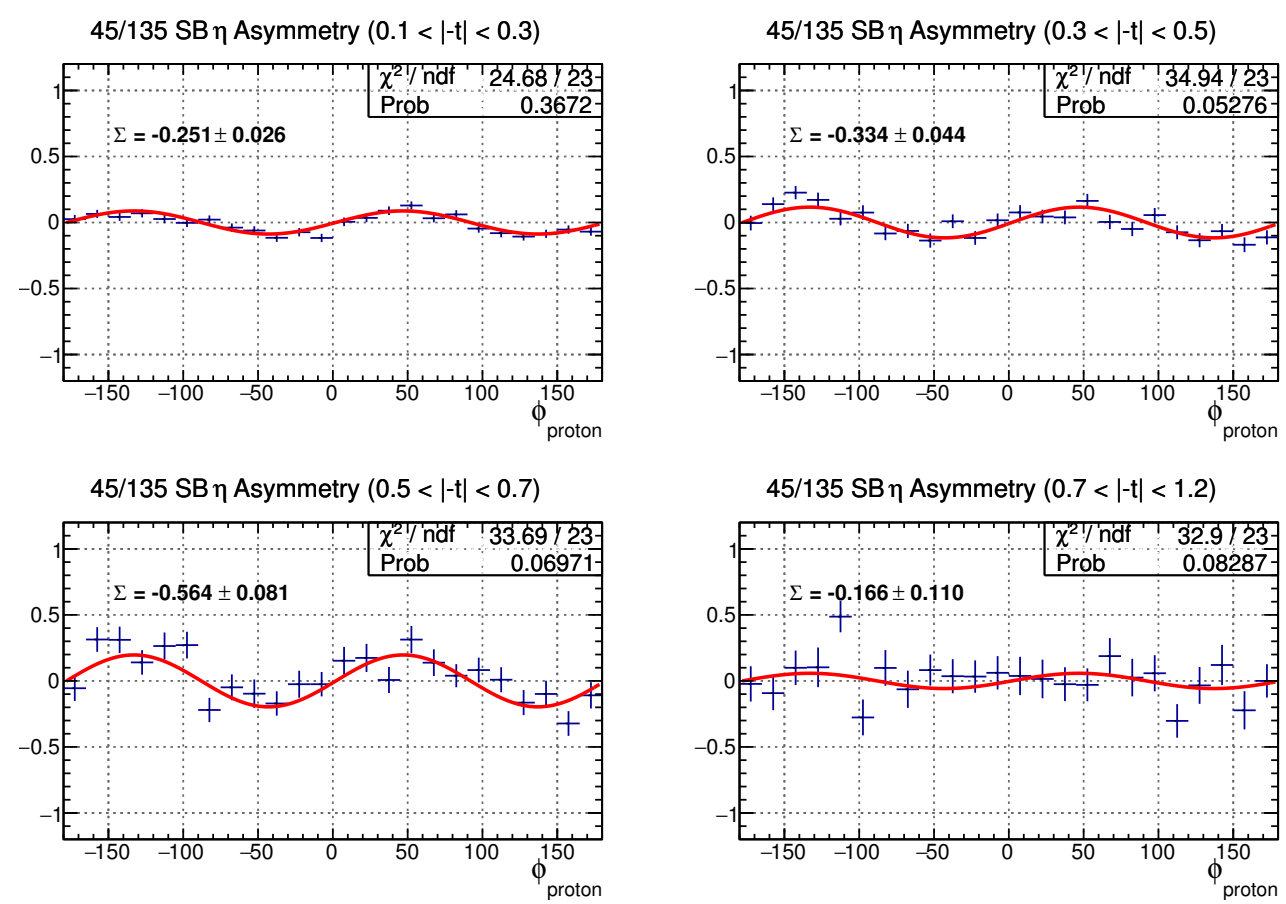

Figure 4.17: $\quad \eta \rightarrow 2 \gamma 45 / 135$ yield asymmetry distributions in the side-band region for four ranges of $t$. 
The similar plots for the 45/135 data set are shown in Figures 4.16 and 4.17. Figure 4.18 shows $\Sigma_{\text {peak }}, \Sigma_{\mathrm{SB}}$, and $\Sigma_{\mathrm{COR}}$ versus $t$ for the $0 / 90$ and $45 / 135$ data sets.
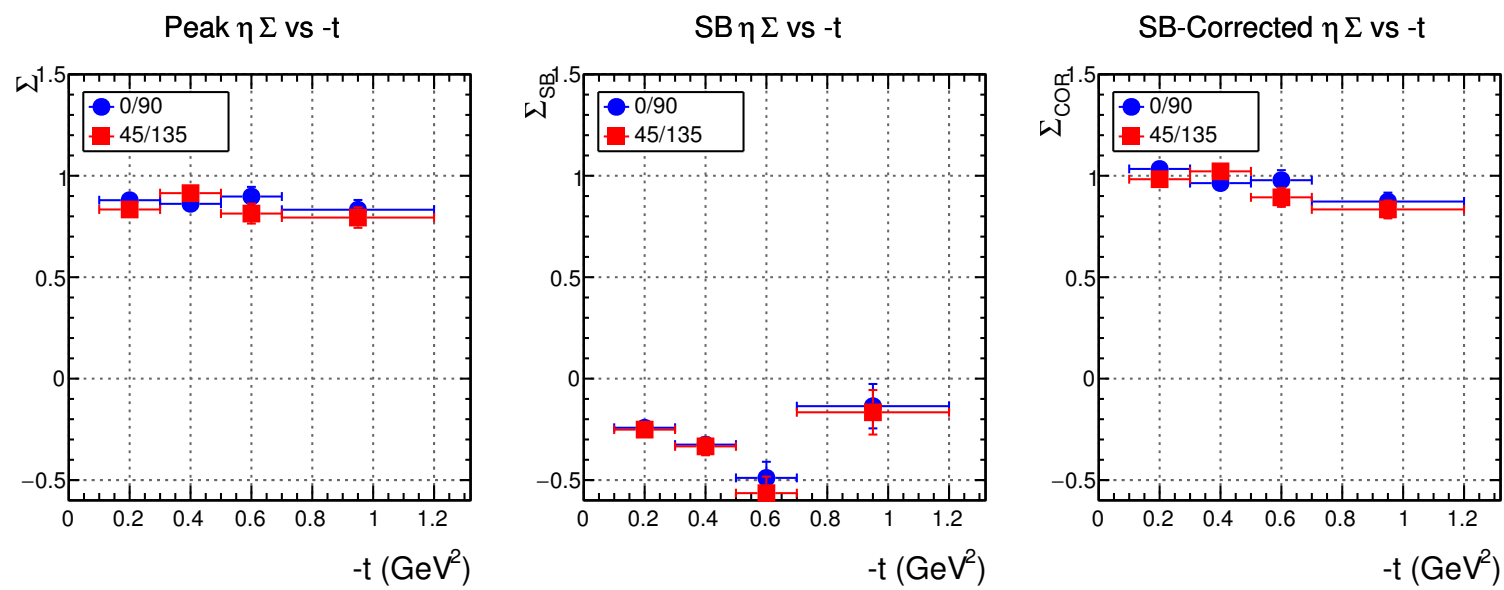

Figure 4.18: $\eta$ beam asymmetries versus $t$ for the $\eta \rightarrow 2 \gamma$ decay channel, from left to right: $\Sigma_{\text {peak }}, \Sigma_{\mathrm{SB}}$, and $\Sigma_{\mathrm{COR}}$. $0 / 90$ data are in blue (circles), 45/135 data are in red (squares). Errors are statistical only (colour online).

Once more, the side-band asymmetries are negative, meaning that the corrected asymmetries are again larger than the peak asymmetries. Since the dilution factors are sizeable, the corrections in this channel are much more noticeable in the lower $-t$ bins, bringing the asymmetry in the lowest $-t$ bin up from $\sim 0.842$ to $\sim 0.990$. The corrections in the higher $-t$ bins are not as noticeable.

The $p$-value for the $\eta \rightarrow 2 \gamma 0 / 90$ and $45 / 135$ asymmetries is 0.304 , which is much larger than 0.05, so the average is calculated. The weighted average between the 0/90 and 45/135 corrected asymmetries is shown in Figure 4.19 and tabulated in Table 4.3. The vertical error bars are statistical only. Here, there is a pronounced $t$-dependence of the $\eta$ beam asymmetry. At low $-t$, the asymmetry is near unity, dropping down close to 0.8 at $t \sim 1 \mathrm{GeV}^{2}$. The errors on these asymmetries are rather small, owing to the large statistics in this channel. Though the asymmetry results from the 3piq and 3 pi0 channels do not clearly show the same trend versus $t$, they are statistically consistent with the results from this high-statistics decay channel. 


\section{$\eta \Sigma$ vs -t}

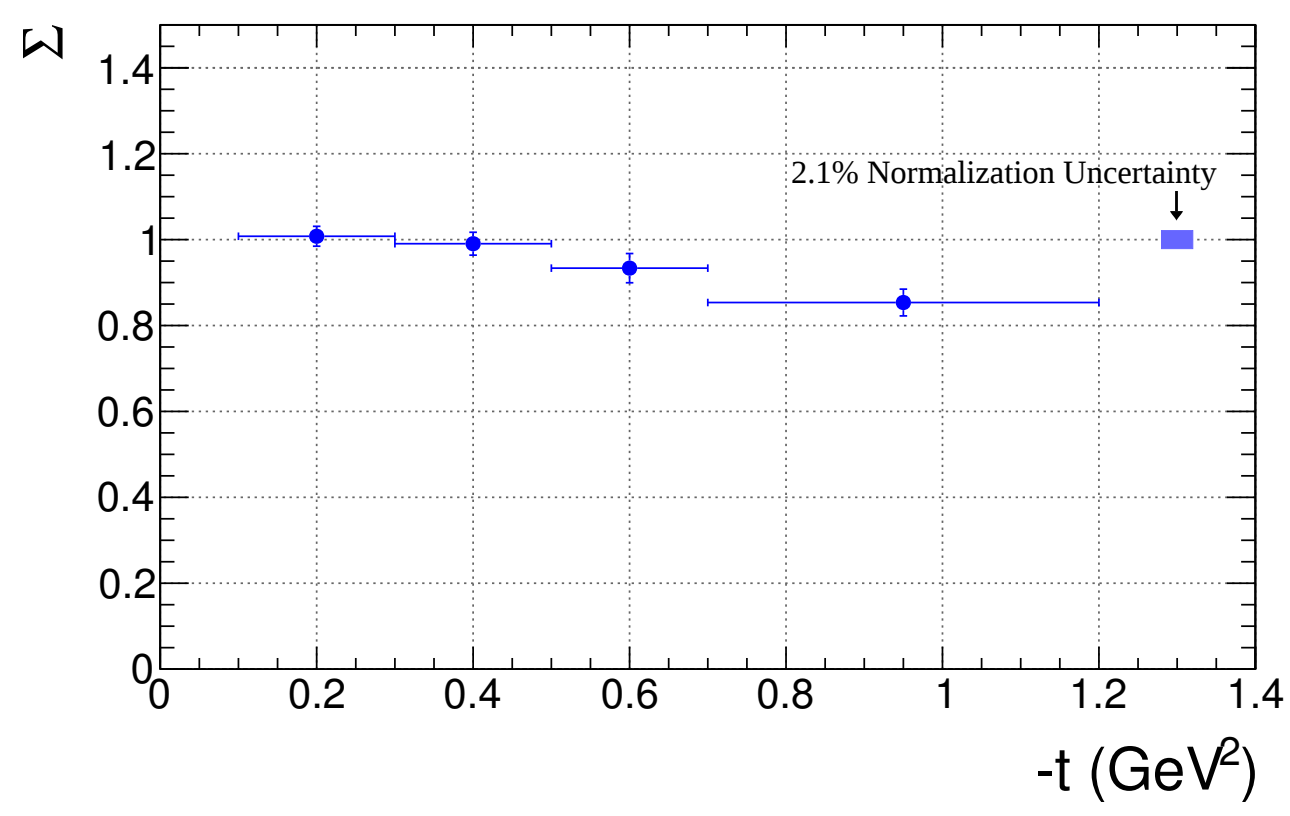

Figure 4.19: Weighted average of 0/90 and 45/135 side-band corrected beam asymmetries for the $\eta \rightarrow 2 \gamma$ decay channel. Vertical error bars are statistical only, and the height of the marked box represents the $2.1 \%$ normalization uncertainty.

\begin{tabular}{|c||c|c|}
\hline$t$ Range $\left(\mathrm{GeV}^{2}\right)$ & $\Sigma_{\text {AVG }}$ & $\delta_{\Sigma_{\text {AVG }}}$ \\
\hline \hline $0.1<-t<0.3$ & 1.008 & 0.023 \\
\hline $0.3<-t<0.5$ & 0.990 & 0.027 \\
\hline $0.5<-t<0.7$ & 0.934 & 0.034 \\
\hline $0.7<-t<1.2$ & 0.854 & 0.031 \\
\hline
\end{tabular}

Table 4.3: Average asymmetries and statistical errors for the $\eta \rightarrow 2 \gamma$ decay channel.

\subsubsection{Past Results and Models}

The $\Sigma$ beam asymmetry results from the three decay channels of the $\eta$ are plotted together in Figure 4.20 (with vertical error bars representing statistical errors), overlayed with the GlueX results published in 2017 on the $\eta \rightarrow 2 \gamma$ beam asymmetry (with vertical error bars representing total errors, and grey boxes representing the systematic error contribution) and the theoretical models from Figure 1.8 (b). The past GlueX results and the new results from the analyses in this thesis suggest that the beam asymmetry of the $\eta$ is highly positive over the entire tested $t$ range, contrary 


\section{$\Sigma$ Beam Asymmetry vs. $-\mathrm{t}$}

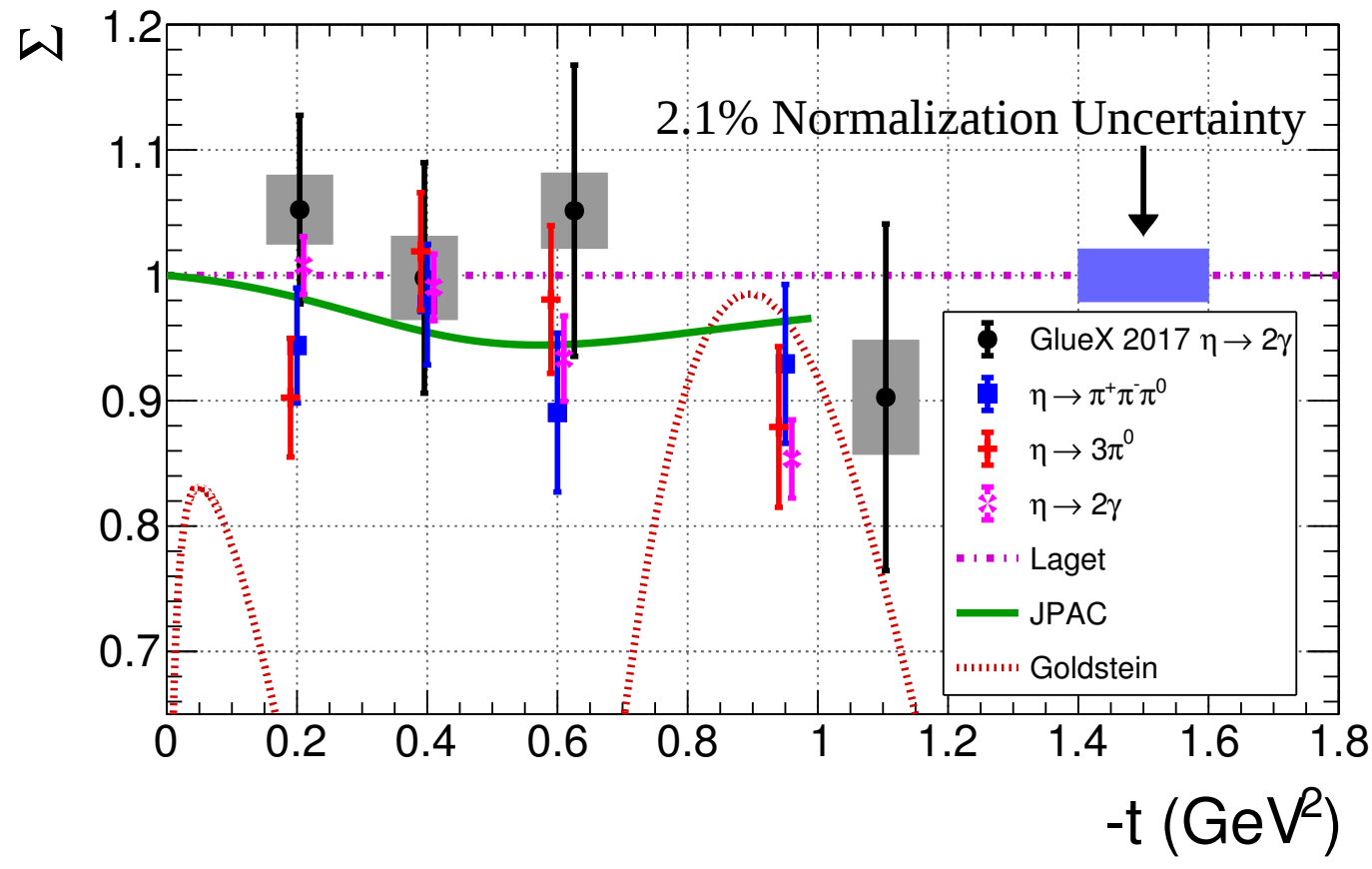

Figure 4.20: $\quad \eta$ beam asymmetries versus $t$ for all three $\eta$ decay channels studied in this thesis (with vertical error bars representing statistical errors and the height of the marked box representing the $2.1 \%$ normalization uncertainty) overlayed with past (published 2017) GlueX results (with vertical error bars representing total errors and grey boxes representing contributions from systematic errors) and the theoretical models from Figure 1.8 (colour online).

to the model prediction from Goldstein, which features a dip around $t=-0.5 \mathrm{GeV}^{2}$. Around $t=1 \mathrm{GeV}^{2}$, the theoretical predictions from the JPAC theory group are consistent with the 2017 GlueX results, but some discrepancy is seen with the new results. In particular, the $\eta \rightarrow 2 \gamma$ asymmetry presented in this thesis lies at around 0.8 at high $-t$, with a much smaller statistical error than the GlueX results published in 2017 achieved.

Following the $\chi^{2}$ test used for combining $0 / 90$ and 45/135 results in each decay channel, the $\chi^{2}$ test statistic (Equation 4.1) and $p$-value are used to quantify the difference between the final asymmetry results from the three $\eta$ decay channels. The two lower-statistics channels (3piq and 3pi0) are compared to the high-statistics $2 \mathrm{~g}$ 


\section{$\Sigma$ Beam Asymmetry vs. - $\mathrm{t}$}

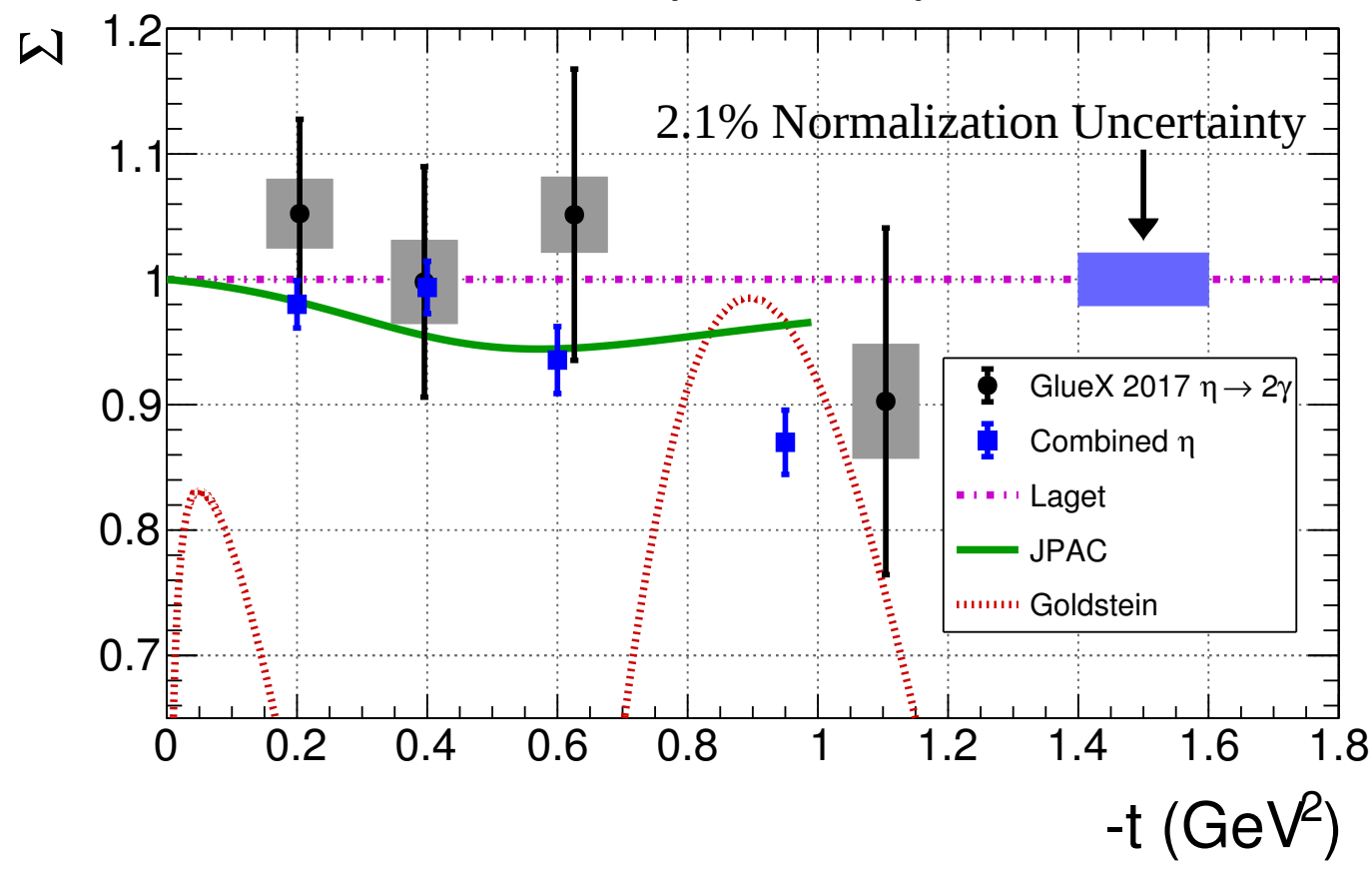

Figure 4.21: $\eta$ beam asymmetries versus $t$ for the weighted average of the three $\eta$ decay channels studied in this thesis (with vertical error bars representing statistical errors and the height of the marked box representing the $2.1 \%$ normalization uncertainty) overlayed with past (published 2017) GlueX results (with vertical error bars representing total errors and grey boxes representing contributions from systematic errors) and the theoretical models from Figure 1.8 (colour online).

channel. The $p$-value found by comparing the 3piq (3pi0) results to the $2 \mathrm{~g}$ results is $0.438(0.182)$. Since both $p$-values are much larger than 0.05 , the distributions are averaged. Figure 4.21 shows the theoretical predictions, the GlueX results published in 2017, and the weighted average (calculated following the structure of Equation 4.3) of the results for the three $\eta$ decay channels. These averages are tabulated in Table 4.4.

\begin{tabular}{|c||c|c|}
\hline$t$ Range $\left(\mathrm{GeV}^{2}\right)$ & $\Sigma_{\eta}$ & $\delta_{\Sigma_{\eta}}$ \\
\hline \hline $0.1<-t<0.3$ & 0.980 & 0.019 \\
\hline $0.3<-t<0.5$ & 0.994 & 0.021 \\
\hline $0.5<-t<0.7$ & 0.936 & 0.027 \\
\hline $0.7<-t<1.2$ & 0.870 & 0.026 \\
\hline
\end{tabular}

Table 4.4: Weighted averages and statistical errors of the three $\eta$ decay channels studied in this thesis. 


\section{$4.2 \quad \eta^{\prime}$ Beam Asymmetry}

In addition to the charged $\eta^{\prime}$ decay channel, analysis of the $\Sigma$ beam asymmetry through two other decay channels of the $\eta^{\prime}$ was attempted. These were the two similar final states as the neutral $\eta$ channels analyzed: $\eta^{\prime} \rightarrow \pi^{0} \pi^{0} \eta, \eta \rightarrow 2 \gamma$ and $\eta^{\prime} \rightarrow 2 \gamma$. Event selection and analysis was done as prescribed in Chapter 3, but it proved impossible to obtain clean, pure event signals with high enough statistics to perform the asymmetry analysis. As such, analysis of these channels using spring 2017 data was abandoned, but will be pursued by others in the collaboration once more data have been calibrated and are available for physics analyses.

\section{$2 \pi^{0} \eta$ Mass Post-Cut}

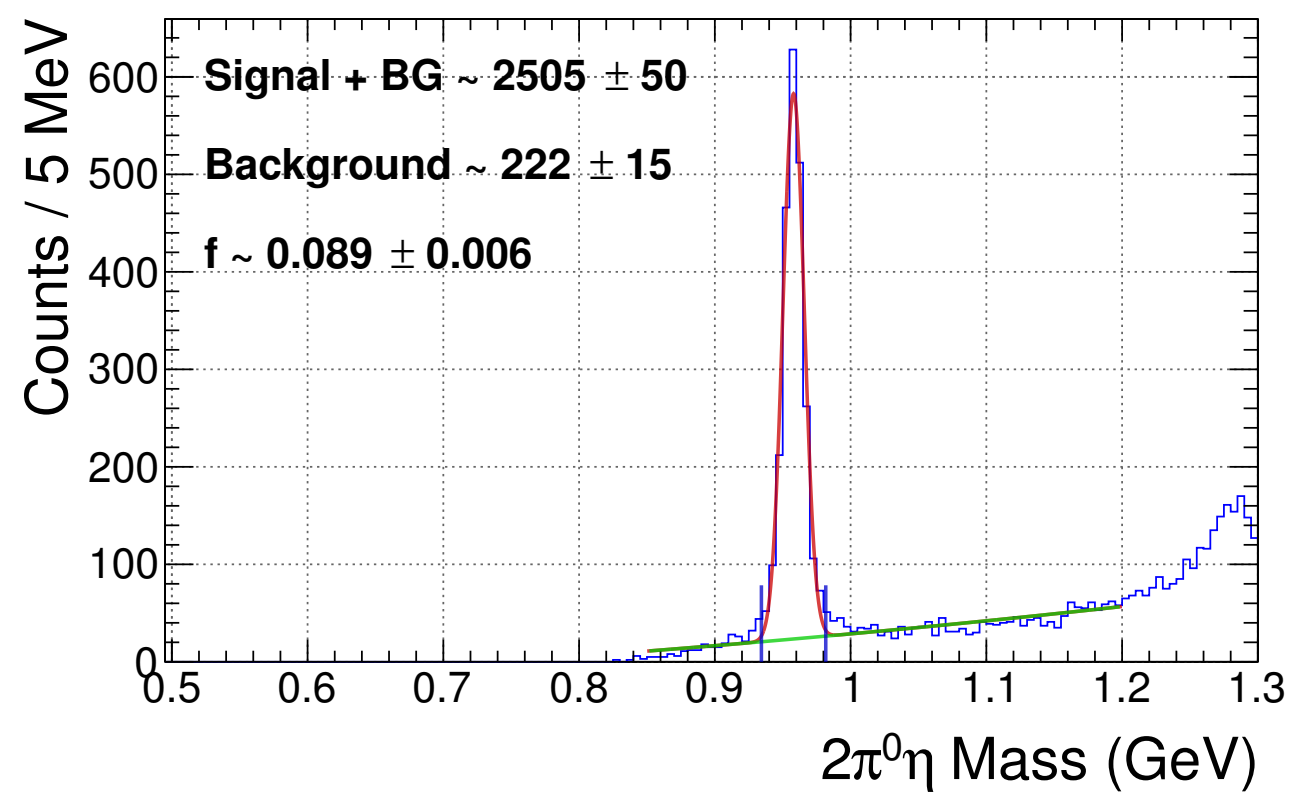

Figure 4.22: $\pi^{0} \pi^{0} \eta$ mass spectrum (all $t$, all polarization orientations).

Figure 4.22 shows the invariant mass spectrum obtained for the $\eta^{\prime} \rightarrow \pi^{0} \pi^{0} \eta$ decay. The primary problem experienced with this channel was the lack of statistics. The reason for the lack of statistics is unknown, however. Based on a collaborator's work on this channel using spring 2016 data, in which nearly 2,000 $\eta^{\prime}$ events were seen in this channel [111], the higher-statistics spring 2017 data set should have yielded many 
more events. After investigation of the event selection process revealed no obvious errors, it was decided to cease analysis of this channel for the time being ${ }^{1}$.

\section{$2 \gamma$ Mass Post-Cut}

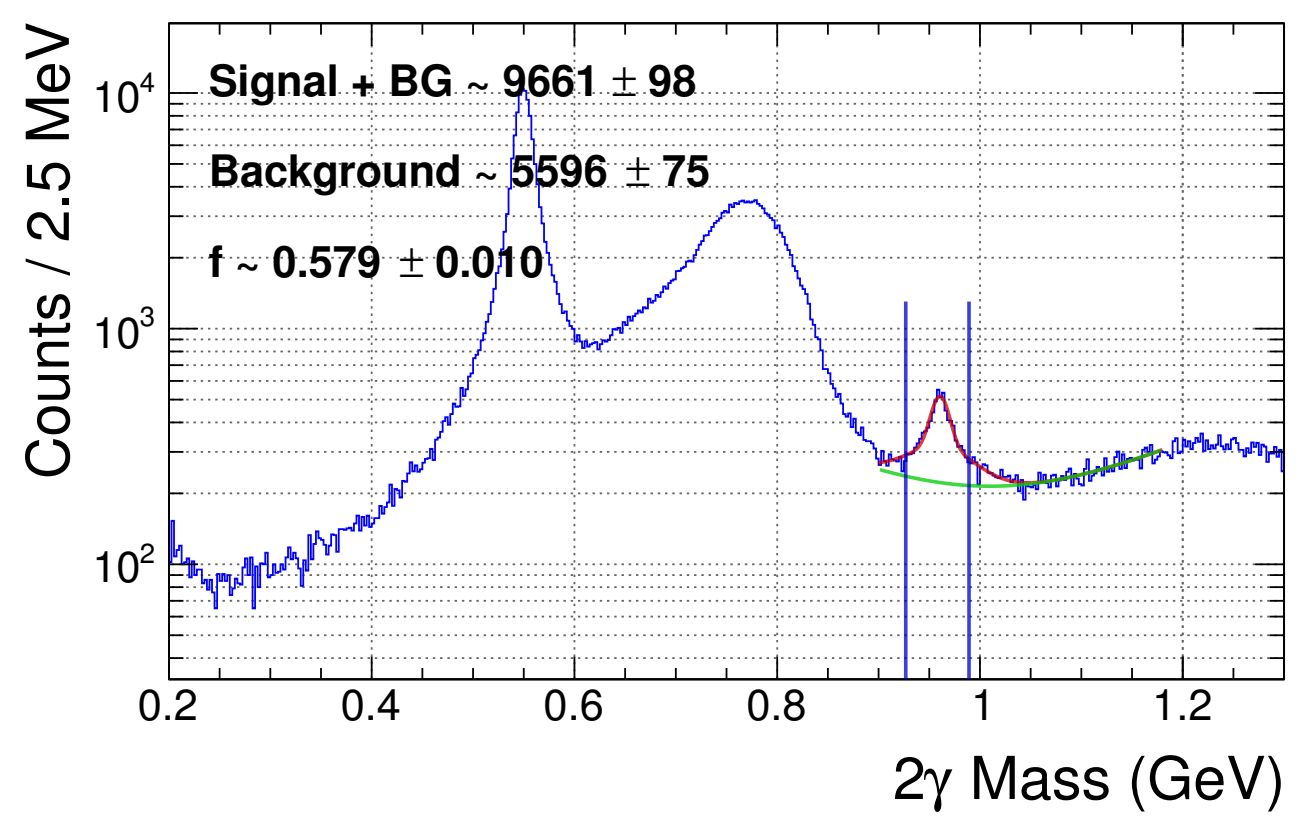

Figure 4.23: $2 \gamma$ mass spectrum, from the decay of the $\eta^{\prime}$, (all $t$, all polarization orientations).

Figure 4.23 shows the invariant mass spectrum obtained for the $\eta^{\prime} \rightarrow 2 \gamma$ decay. The problem with this channel, from the perspective of asymmetry analysis, is the contribution to the background coming from both the low- and high-mass side-bands. The large peak at lower mass is mostly due to the $\omega \rightarrow \pi^{0} \gamma$ decay with a missing photon (the same background as seen in the $2 \gamma$ channel of the $\eta$ ), but there is also a continuum background above the $\eta^{\prime}$ mass. Attempts were made to eliminate more of the background to improve the clarity of the signal, but these efforts were not able to adequately isolate the signal peak. Applying multiple side-band corrections, one for the $\omega$ peak and one for the higher-mass continuum, was considered, but the difficulties involved in fitting two separate backgrounds for two corrections coupled

\footnotetext{
${ }^{1}$ Discrepancies between all-neutral channels and those with both charged and neutral particles have been seen elsewhere. The GlueX Calorimetry Working Group has produced a plan to resolve this in 2019.
} 
with relatively low statistics again led to the decision to abandon the asymmetry analysis of this channel for the time being.

In contrast, the $\eta^{\prime} \rightarrow \pi^{+} \pi^{-} \eta$ channel provides a clean signal with very little background under the peak. In addition, the background seems to come from a continuum that extends to higher masses and has sizeable statistics above $1.05 \mathrm{GeV}$, which makes doing a side-band asymmetry correction possible.
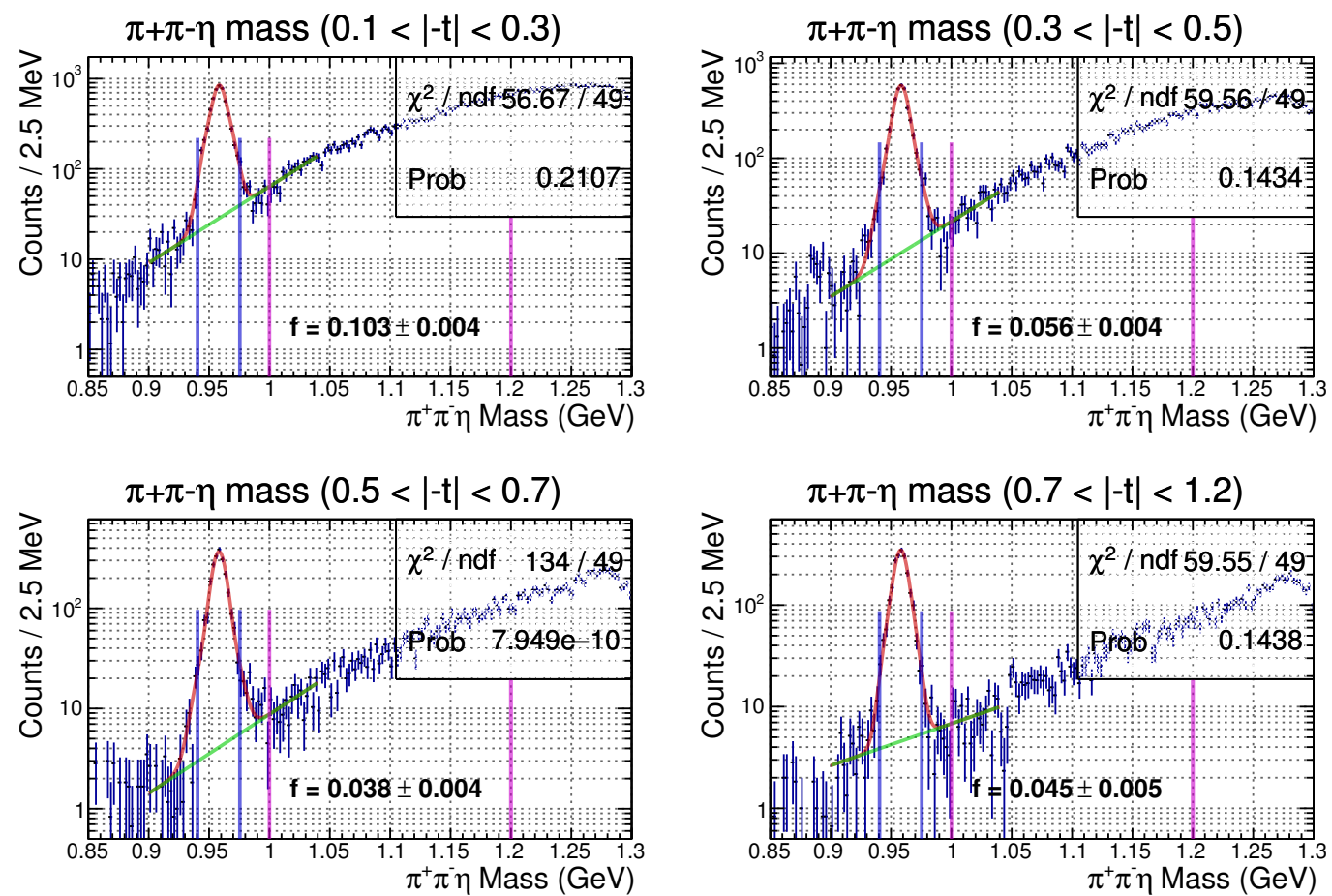

Figure 4.24: $\pi^{+} \pi^{-} \eta$ mass spectra and dilution factors for four ranges of $t$ (all polarization orientations). The blue delimiting lines denote the peak region, and the purple delimiting lines denote the side-band region (colour online).

\subsection{1 $\eta^{\prime} \rightarrow \pi^{+} \pi^{-} \eta, \eta \rightarrow 2 \gamma$}

Figure 4.24 shows the $\pi^{+} \pi^{-} \eta$ mass spectra (binned in $t$ ). The statistics in this channel are comparable to the 3piq channel, and the amount of background under the signal peak (between $3.8 \%$ and 10.3\%) is similarly small, though not necessarily insignificant, so side-band corrections are carried out. Though the background is not 
perfectly described by an exponential up to high mass, because of contributions from multiple unknown sources and the $f_{1}(1285)$ peak, the exponential function adequately describes the background in the immediate vicinity of the $\eta^{\prime}$ peak. The side-band region is chosen as the region in mass between the $\eta^{\prime}$ and $f_{1}(1285)$ peaks, from $1.0 \mathrm{GeV}$ to $1.2 \mathrm{GeV}$. The $f_{1}(1285)$ peak is omitted from the side-band because it is unlikely that any true $f_{1}(1285)$ events contribute to the background under the signal peak. The assumption that the side-band asymmetry is mass-independent is tested in Section 5.2.1. The dilution factors obtained for each fit are displayed on the plots, along with the peak region (defined by the width of the larger of the two Gaussian widths from the $t$-averaged fit, shown previously in Figure 3.7) and side-band region.
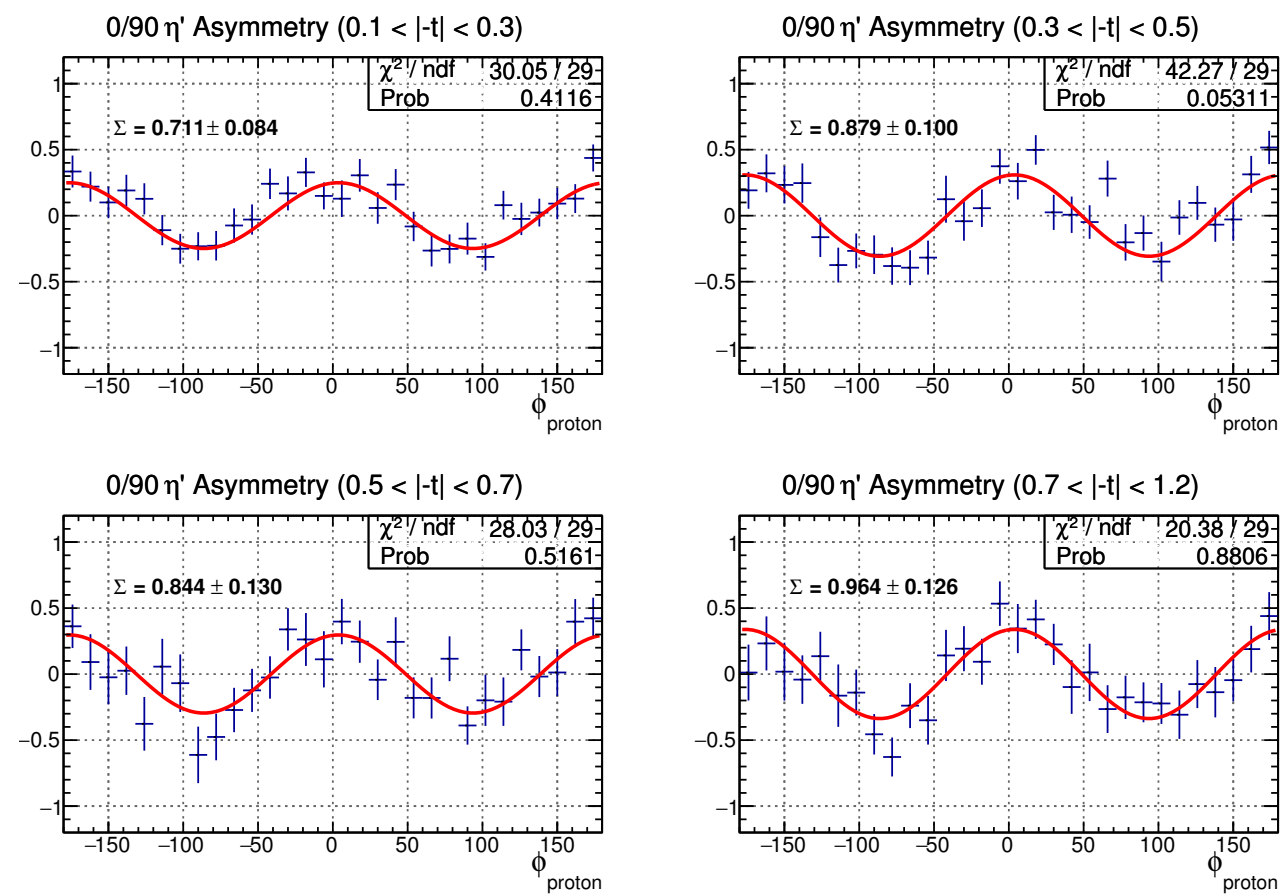

Figure 4.25: $\quad \eta^{\prime} \rightarrow \pi^{+} \pi^{-} \eta 0 / 90$ yield asymmetry distributions in the $\eta^{\prime}$ peak region for four ranges of $t$.

Figures 4.25 and 4.26 show the accidental-subtracted yield asymmetry $\phi$ distributions for the $\eta^{\prime}$ peak region and side-band region (0/90 data set). 

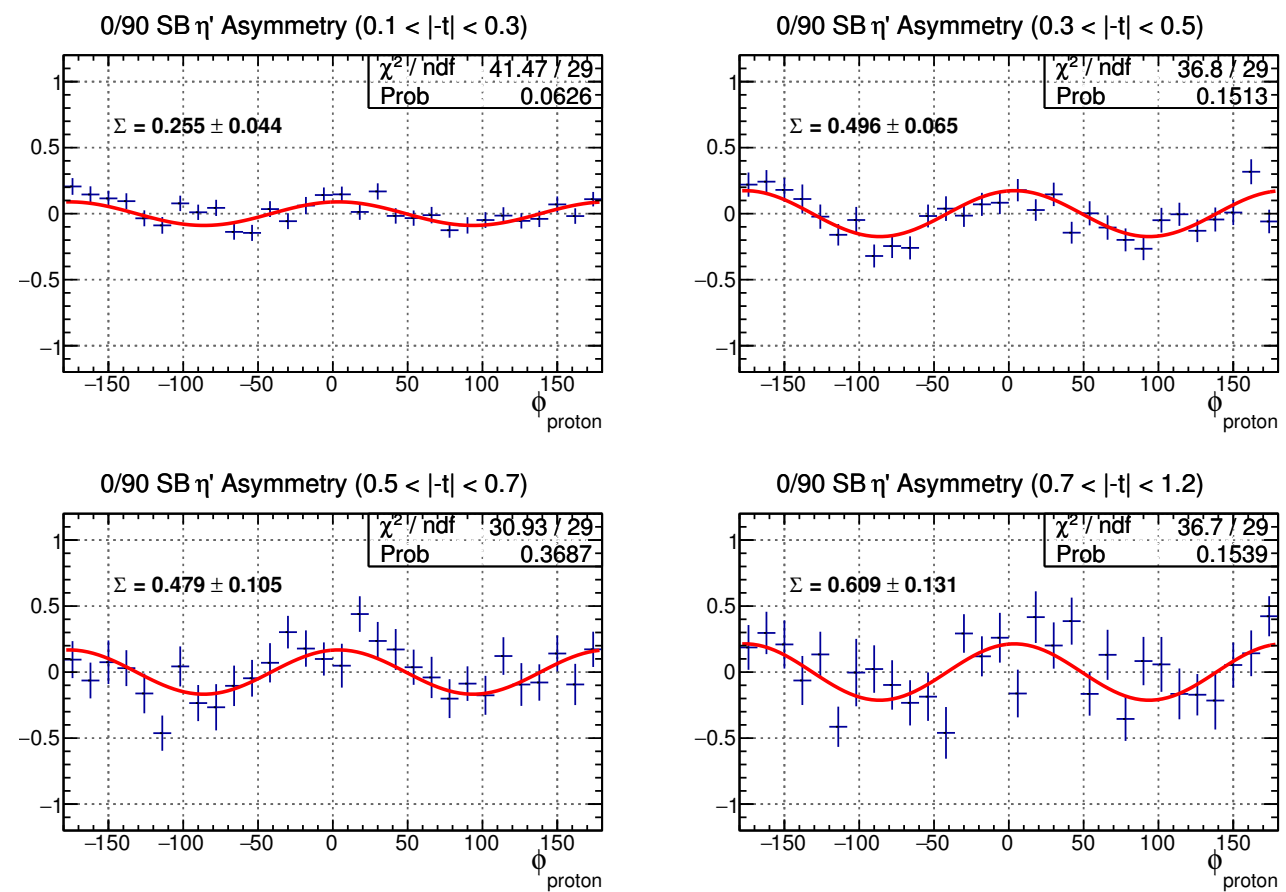

Figure 4.26: $\eta^{\prime} \rightarrow \pi^{+} \pi^{-} \eta 0 / 90$ yield asymmetry distributions in the side-band region for four ranges of $t$.
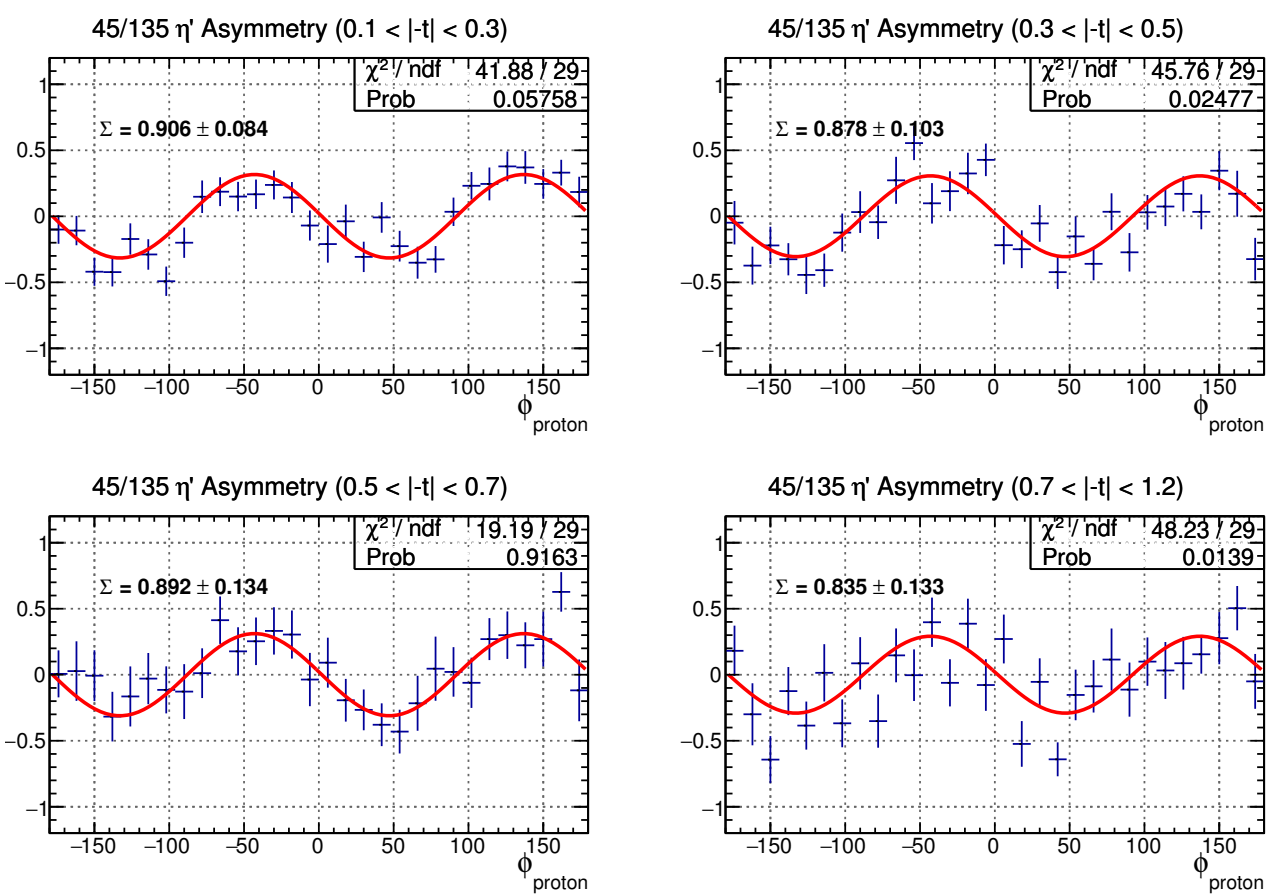

Figure 4.27: $\eta^{\prime} \rightarrow \pi^{+} \pi^{-} \eta$ 45/135 yield asymmetry distributions in the $\eta^{\prime}$ peak region for four ranges of $t$. 

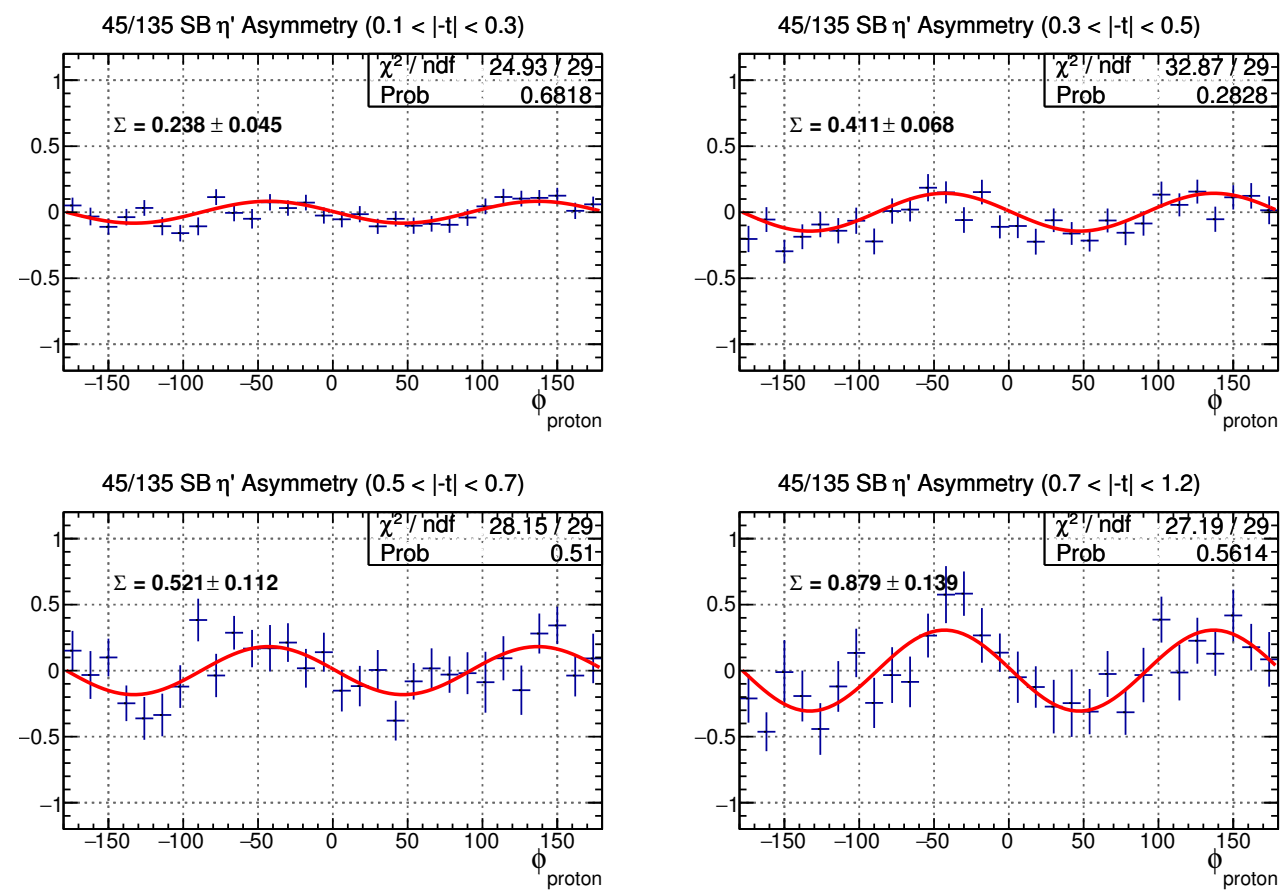

Figure 4.28: $\quad \eta^{\prime} \rightarrow \pi^{+} \pi^{-} \eta 45 / 135$ yield asymmetry distributions in the side-band region for four ranges of $t$.

The similar plots for the 45/135 data set are shown in Figures 4.27 and 4.28. Figure 4.29 shows $\Sigma_{\text {peak }}, \Sigma_{\mathrm{SB}}$, and $\Sigma_{\mathrm{COR}}$ versus $t$ for both the $0 / 90$ and $45 / 135$ data sets.
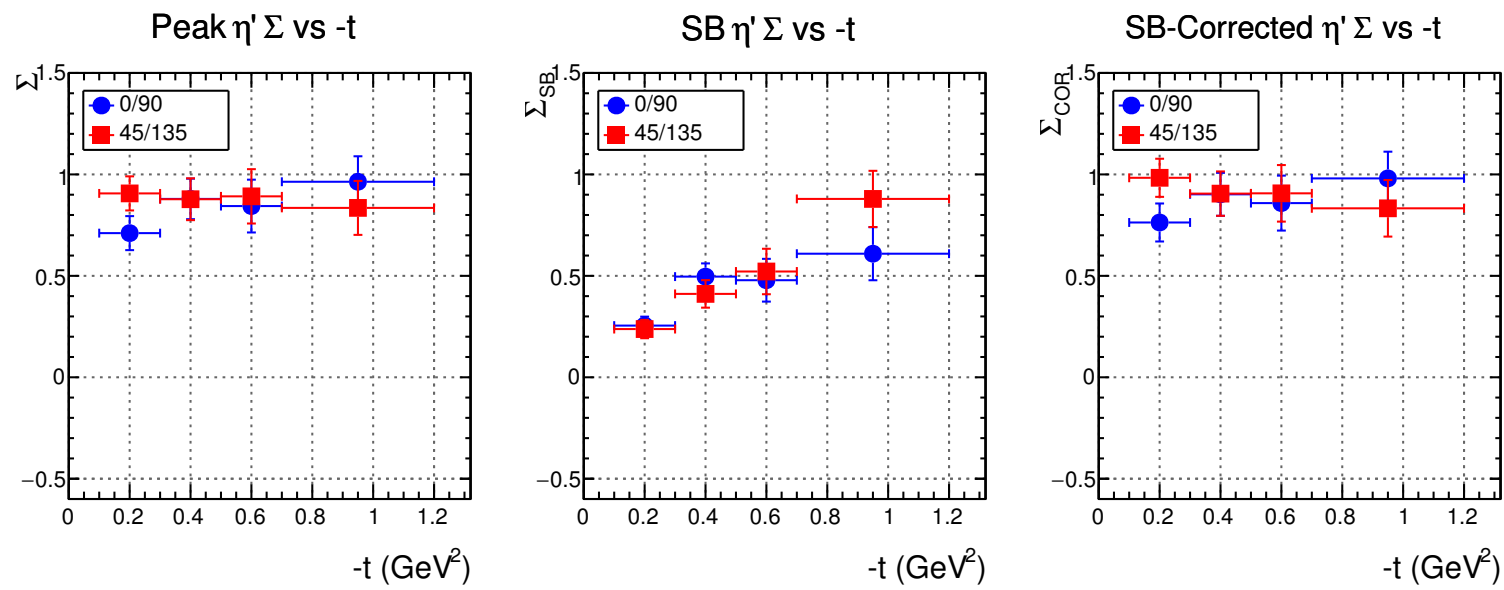

Figure 4.29: $\eta^{\prime}$ beam asymmetries versus $t$ for the $\eta^{\prime} \rightarrow \pi^{+} \pi^{-} \eta$ decay, from left to right: $\Sigma_{\text {peak }}, \Sigma_{\mathrm{SB}}$, and $\Sigma_{\mathrm{COR}}$. 0/90 data are in blue (circles), 45/135 data are in red (squares). Errors are statistical only (colour online). 
Contrary to the $\eta$ channels, the side-band asymmetry in the $\eta^{\prime}$ channel is positive. Because the side-band and peak asymmetries are relatively close in magnitude, and because the dilution factors are small, the side-band corrections in this channel are practically unnoticeable at high $-t$ and rather small at low $-t$. Again in contrast to the $\eta$ channels, wherein the side-band consisted of the peaking structure of the $\omega$, the side-band here is a continuum background with contributions from different decay channels. Since inclusion of the $f_{1}$ peak in the side-band region is avoided, the side-band in higher $-t$ bins carry low statistics ${ }^{2}$.

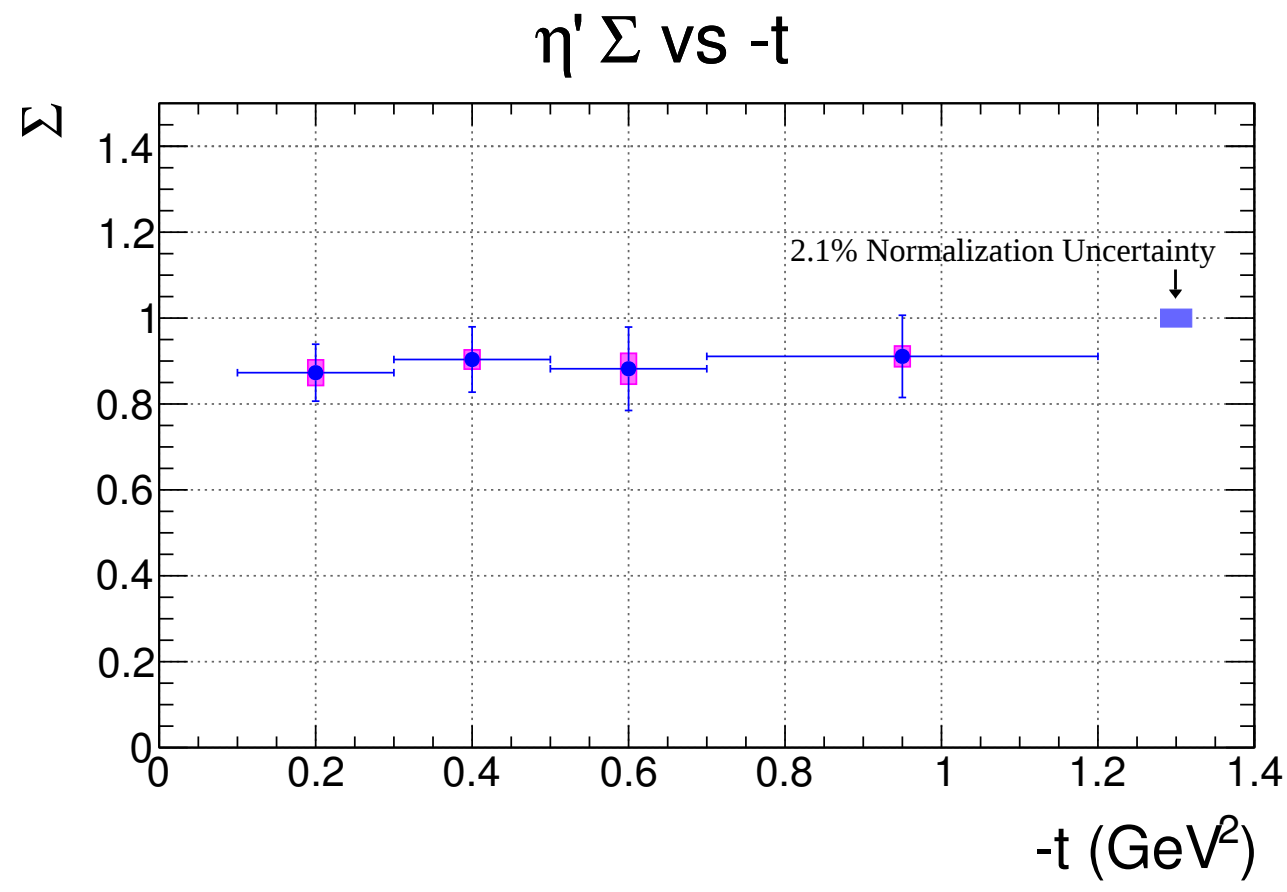

Figure 4.30: Weighted average of $0 / 90$ and 45/135 side-band corrected beam asymmetries for the $\eta^{\prime} \rightarrow \pi^{+} \pi^{-} \eta$ decay channel. Vertical error bars are statistical only, and the height of the marked box represents the $2.1 \%$ normalization uncertainty. The purple boxes on each point indicate the systematic error assigned to that point (colour online).

The $p$-value for the $\eta^{\prime} \rightarrow \pi^{+} \pi^{-} \eta 0 / 90$ and $45 / 135$ asymmetries is 0.397 . Since this is much larger than the significance level of 0.05 , the distributions are not found to be statistically different and the results are averaged. The weighted average between

\footnotetext{
${ }^{2}$ This is the driving factor in the choice of the number of $t$ bins for the $\eta$ channels. With the highstatistics $\eta \rightarrow 2 \gamma$ channel, finer binning could be used, but in order to smoothly compare between all four studied decay channels, it was decided to use the same binning for each analysis.
} 


\begin{tabular}{|c||c|c|c|}
\hline$t$ Range $\left(\mathrm{GeV}^{2}\right)$ & $\Sigma_{\mathrm{AVG}}$ & $\delta_{\Sigma_{\mathrm{AVG}}}$ & $\delta_{\mathrm{SYS}}$ \\
\hline \hline $0.1<-t<0.3$ & 0.873 & 0.066 & 0.030 \\
\hline $0.3<-t<0.5$ & 0.904 & 0.076 & 0.022 \\
\hline $0.5<-t<0.7$ & 0.882 & 0.097 & 0.036 \\
\hline $0.7<-t<1.2$ & 0.911 & 0.096 & 0.024 \\
\hline
\end{tabular}

Table 4.5: Average asymmetries, statistical errors, and systematic errors for the $\eta^{\prime} \rightarrow \pi^{+} \pi^{-} \eta$ decay channel. The sources of the systematic errors are discussed in Section 5.2.

the $0 / 90$ and $45 / 135$ corrected asymmetries is shown in Figure 4.30 and tabulated in Table 4.5. The vertical error bars are statistical only. An estimate of the systematic error on the average asymmetries is shown as purple boxes on each data point in Figure 4.30. The sources and analysis of this systematic error is discussed in Section 5.2. The beam asymmetry in this $\eta^{\prime}$ decay channel appears to be flat versus $t$ with a similar magnitude as the $\eta$ asymmetry.

\subsection{Beam Asymmetry Ratio}

In order to compare to the JPAC theory curves, which were discussed briefly in Section 1.6.2, the ratio of $\eta$ meson beam asymmetries is formed using the $\eta^{\prime}$ decay channel and each of the $\eta$ decay channels. The ratios of the data set averages are shown in Figure 4.31 along with the JPAC theory curves. The same results are tabulated in Table 4.6.

\begin{tabular}{|c||c|c||c|c||c|c|}
\cline { 2 - 7 } \multicolumn{1}{c|}{} & \multicolumn{2}{c||}{$\eta^{\prime} / \eta \rightarrow 3 \mathrm{piq}$} & \multicolumn{2}{c||}{$\eta^{\prime} / \eta \rightarrow 3 \mathrm{pi0}$} & \multicolumn{2}{c|}{$\eta^{\prime} / \eta \rightarrow 2 \mathrm{~g}$} \\
\hline$t$ range $\left(\mathrm{GeV}^{2}\right)$ & $\Sigma_{\eta^{\prime}} / \Sigma_{\eta}$ & $\delta_{\Sigma_{\eta^{\prime}} / \Sigma_{\eta}}$ & $\Sigma_{\eta^{\prime}} / \Sigma_{\eta}$ & $\delta_{\Sigma_{\eta^{\prime}} / \Sigma_{\eta}}$ & $\Sigma_{\eta^{\prime}} / \Sigma_{\eta}$ & $\delta_{\Sigma_{\eta^{\prime}} / \Sigma_{\eta}}$ \\
\hline \hline $0.1<-t<0.3$ & 0.925 & 0.083 & 0.967 & 0.085 & 0.866 & 0.073 \\
\hline $0.3<-t<0.5$ & 0.925 & 0.090 & 0.887 & 0.089 & 0.912 & 0.082 \\
\hline $0.5<-t<0.7$ & 0.990 & 0.130 & 0.900 & 0.124 & 0.945 & 0.115 \\
\hline $0.7<-t<1.2$ & 0.980 & 0.123 & 1.036 & 0.125 & 1.067 & 0.109 \\
\hline
\end{tabular}

Table 4.6: Asymmetry ratios (of the $\eta^{\prime}$ results to the three $\eta$ results) and associated statistical errors. 


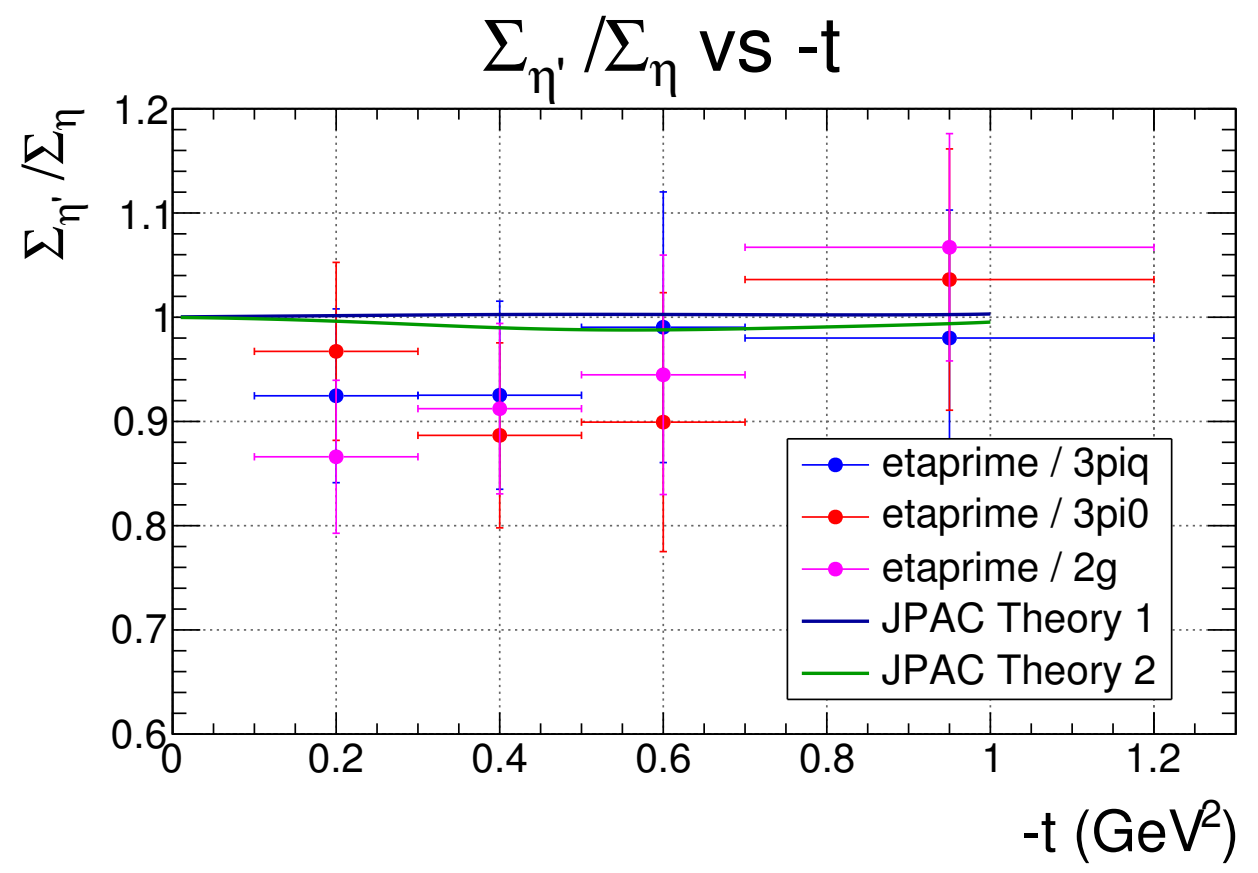

Figure 4.31: $\Sigma_{\eta^{\prime}} / \Sigma_{\eta}$ versus $t$, found as the ratios of data from the $\eta^{\prime}$ decay channel and the three $\eta$ decay channels analyzed. The two theoretical curves from JPAC (from Figure 1.9) are overlayed for direct comparison of data to theory. Errors are statistical only (colour online).

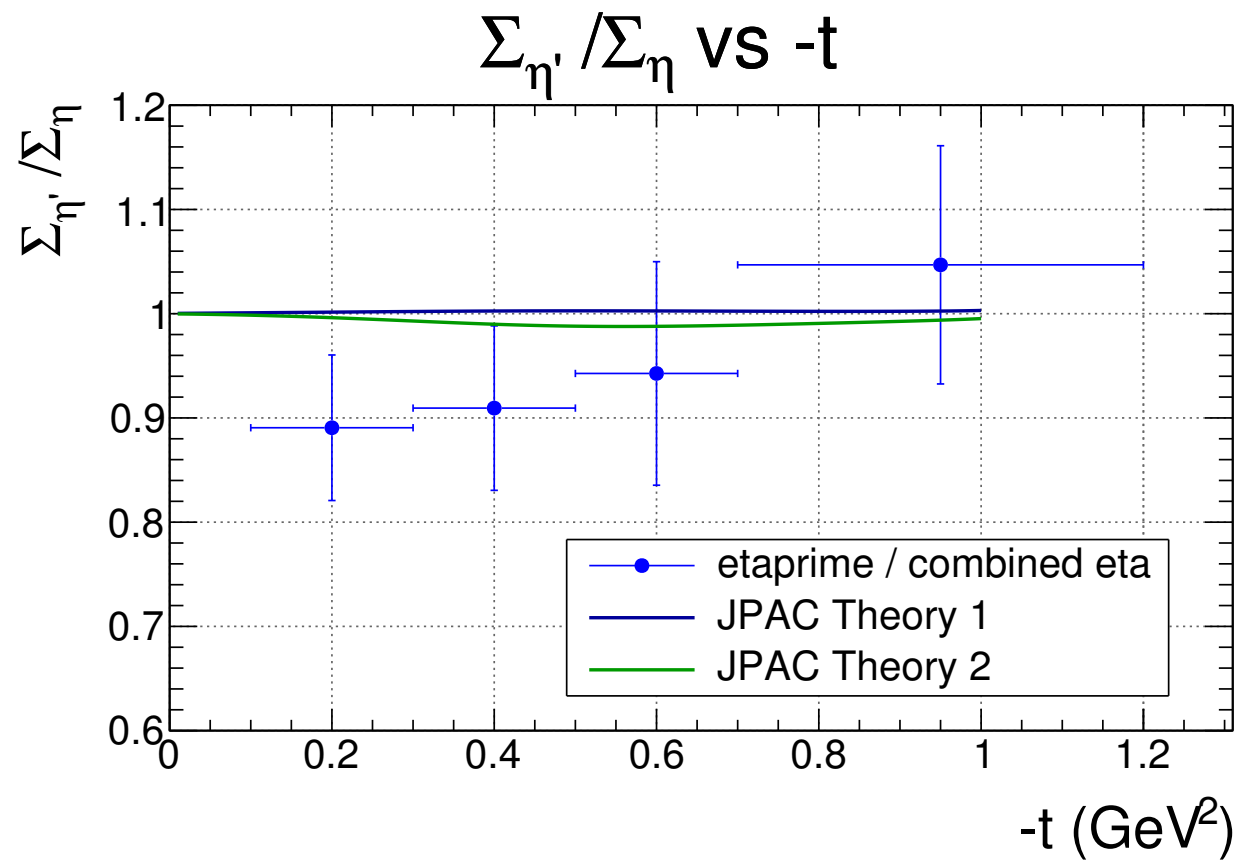

Figure 4.32: $\quad \Sigma_{\eta^{\prime}} / \Sigma_{\eta}$ versus $t$, found as the ratios of data from the $\eta^{\prime}$ decay channel and the weighted average of the three $\eta$ decay channels analyzed. The two theoretical curves from JPAC (from Figure 1.9) are overlayed for direct comparison of data to theory. Errors are statistical only (colour online). 
Finally, the ratio of the $\eta^{\prime}$ decay channel results with the weighted average of the three $\eta$ decay channel results (from Section 4.1.4, Table 4.4) is constructed. This, along with the same JPAC theory curves, is shown in Figure 4.32 and tabulated in Table $4.7^{3}$. With the current analyses, a slight upward trend of the asymmetry ratio with $t$ is observed, and no statistically significant deviation from unity is seen in any bin of $t$ (the largest deviation is $1.5 \sigma$ in the lowest $-t$ bin).

\begin{tabular}{|c||c|c|}
\hline$t$ Range $\left(\mathrm{GeV}^{2}\right)$ & $\Sigma_{\eta^{\prime}} / \Sigma_{\eta}$ & $\delta_{\Sigma_{\eta^{\prime}} / \Sigma_{\eta}}$ \\
\hline \hline $0.1<-t<0.3$ & 0.891 & 0.070 \\
\hline $0.3<-t<0.5$ & 0.909 & 0.079 \\
\hline $0.5<-t<0.7$ & 0.943 & 0.107 \\
\hline $0.7<-t<1.2$ & 1.047 & 0.114 \\
\hline
\end{tabular}

Table 4.7: Asymmetry ratios (of the $\eta^{\prime}$ results to the weighted average of the three $\eta$ results) and associated statistical errors.

Given how close to each other the two theory curves from the JPAC group are, it will not likely be possible to say with certainty if one of the two curves more accurately describes data that will be collected with the GlueX Experiment, but if analysis of more data (from 2018 and 2019 run periods) shows a deviation from both theory curves (an asymmetry ratio in some $t$ bin that shows a statistically significant deviation from unity, for instance), corrections to the theory models may be made.

\footnotetext{
${ }^{3}$ In the ratio of two asymmetry results, any effects due to shifts in the polarizations will cancel, since such shifts will alter both asymmetry results in the same way. As such, no normalization uncertainty is applied to the asymmetry ratios.
} 


\section{Chapter 5}

\section{Systematic Studies}

With the event selection and analysis methods set, a result and statistical error can be extracted. However, it is also necessary to check the systematics of the analysis. This usually involves altering some facet of the analysis or event selection in a controlled way and observing the changes in the results. These studies help to choose the most optimal selection cuts or provide an estimate of the systematic error on the final results.

Often, a selection cut is made based on a two-dimensional plot of data that clearly rejects some background (invariant masses that are far from a true particle mass, for instance), but in some cases, the effects of a cut on a variable are not so obvious. Placing a loose cut to keep the highest number of signal events may also keep a lot of background events, such that the signal-to-background ratio may be too small to extract the physics observable accurately. Placing a tight cut to get the highest signal purity may cut out too many signal events, causing large statistical errors. In these cases, the cut can be varied and the final result calculated for each cut variation to look for an optimal cut.

In a similar vein, assigning a systematic uncertainty on the final result indicates the possible variation of the result as a consequence of the choice of event selection or analysis methods. For example, a cut may be varied from its nominal value to a 
slightly tighter (cutting out more events) or looser (cutting out fewer events) cut to check how much the final result changes. If the changes are small, some confidence is given to the choice of nominal cut.

In this chapter, the study of the kinematic fit CL selection cut is presented and the contributions to the assigned systematic errors on the $\eta^{\prime}$ asymmetry are detailed.

\subsection{Kinematic Fit Confidence Level Cut Study}

As discussed in Section 3.1.2, the kinematic fit produces a CL between 0 and 1 that indicates how likely the particle combo is to be from the specified decay. A lower CL means the event is less likely to be a true signal event. In order to find the optimal CL cut to use, a range of cuts is scanned to investigate the effect on statistics, errors, and fit stability.

For each CL cut, accidental-corrected asymmetries and statistical errors are extracted for both the 0/90 and 45/135 data sets in bins of $t$. For channels needing side-band corrections, the side-band asymmetries are also extracted and the corrected asymmetries calculated. Finally, the averages between the two data sets are formed. Plotting these values versus CL cut provides a look at how the fits vary with statistics and purity. At higher CL cuts, lower statistics affect the fits, but high purity reduces the effects of background contributions. The best choice of CL cut is one where the trend versus CL cut of the corrected asymmetry is relatively flat and the statistical error on the fit is still small.

Results of the CL scans for the $\eta \rightarrow \pi^{+} \pi^{-} \pi^{0}$ channel are shown in Figure 5.1 for the dilution factor, $f$, and Figure 5.2 for the SB-corrected average $\Sigma$ asymmetry. Separate results for the 0/90 and 45/135 SB-corrected asymmetries are shown in Appendix B, Figures B.1 and B.2. Each colour and marker shape corresponds to a $t$ bin, so trends versus CL in each bin can be inspected (see legends in each figure). 


\section{Fractional Background}

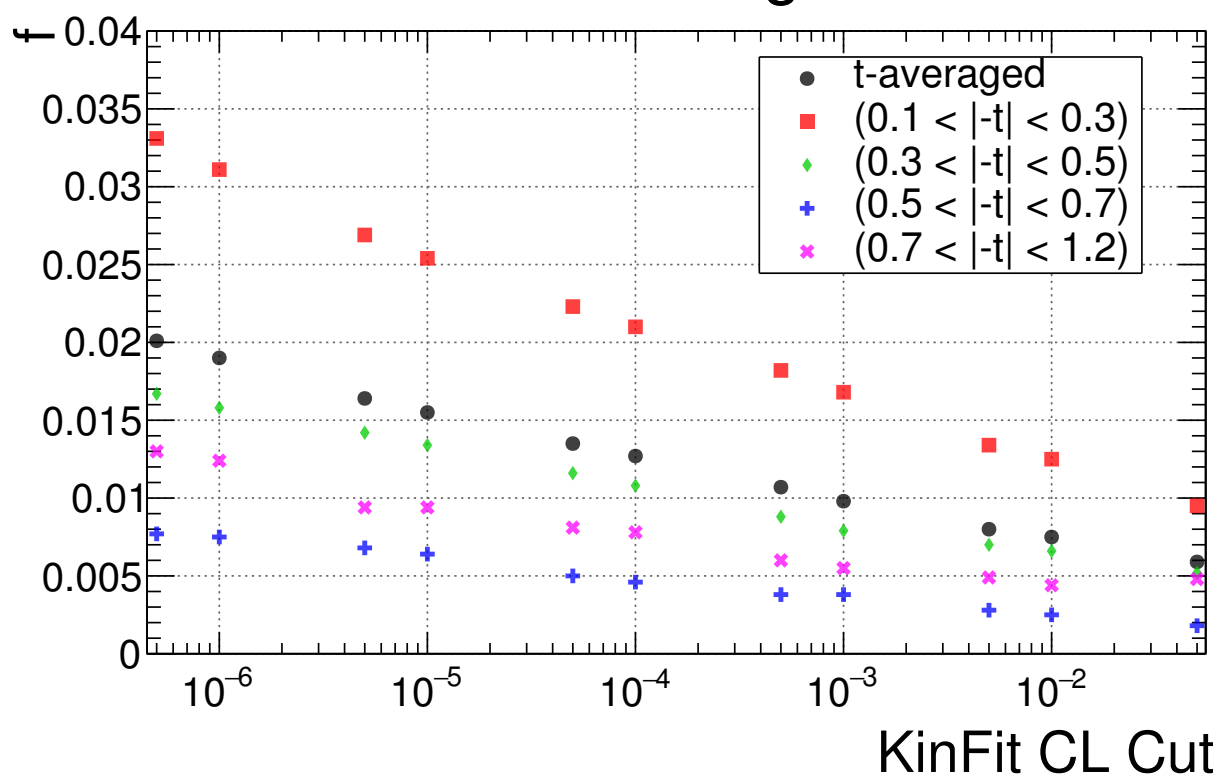

Figure 5.1: $\eta \rightarrow \pi^{+} \pi^{-} \pi^{0}$ dilution factor, $f$, for the studied bins of $t$ versus kinematic fit confidence level cut (colour online).
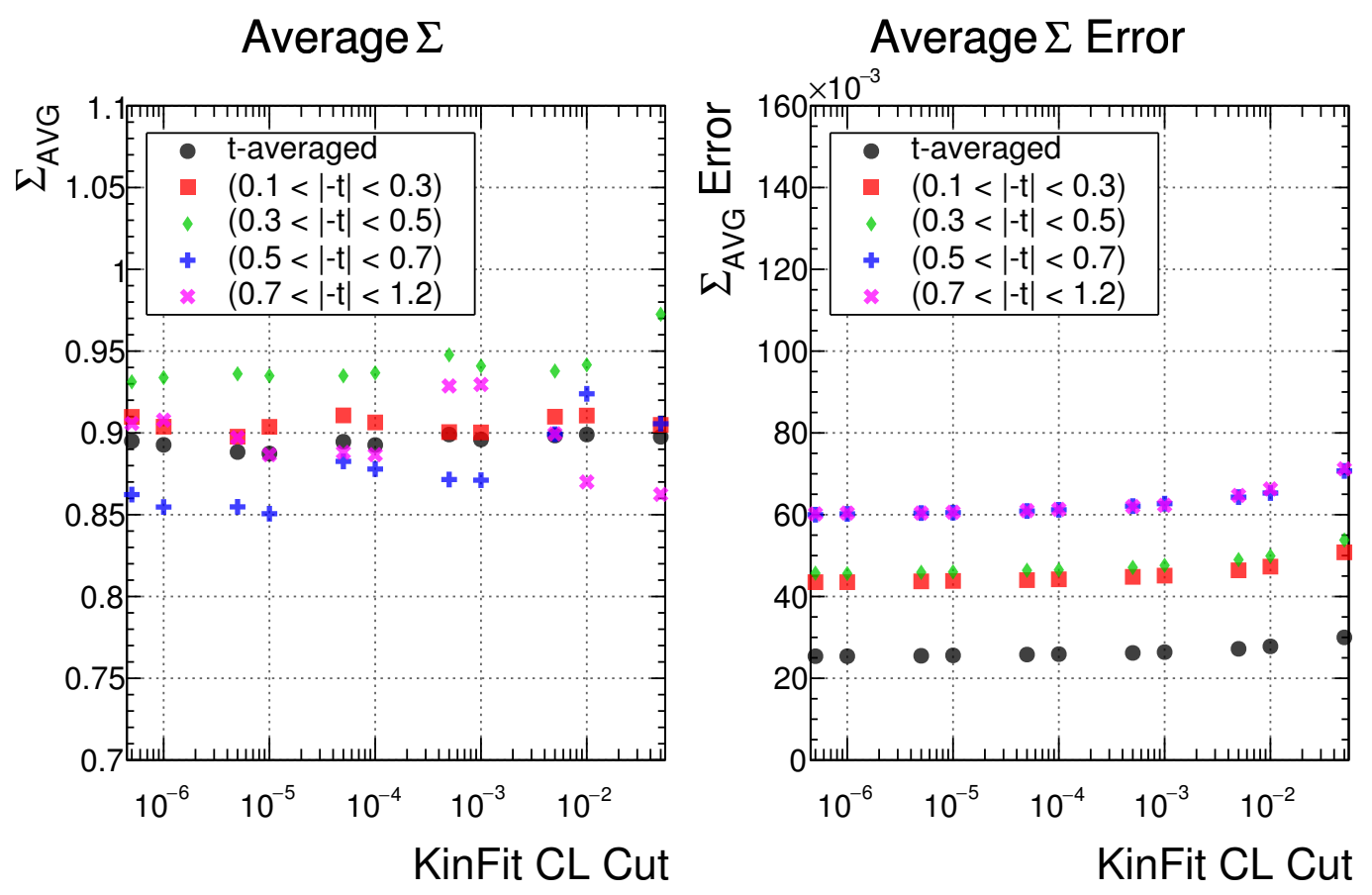

Figure 5.2: $\eta \rightarrow \pi^{+} \pi^{-} \pi^{0}$ average SB-corrected $\Sigma$ asymmetries for the studied bins of $t$ versus kinematic fit confidence level cut (left) and statistical errors on those asymmetries (right) (colour online). 

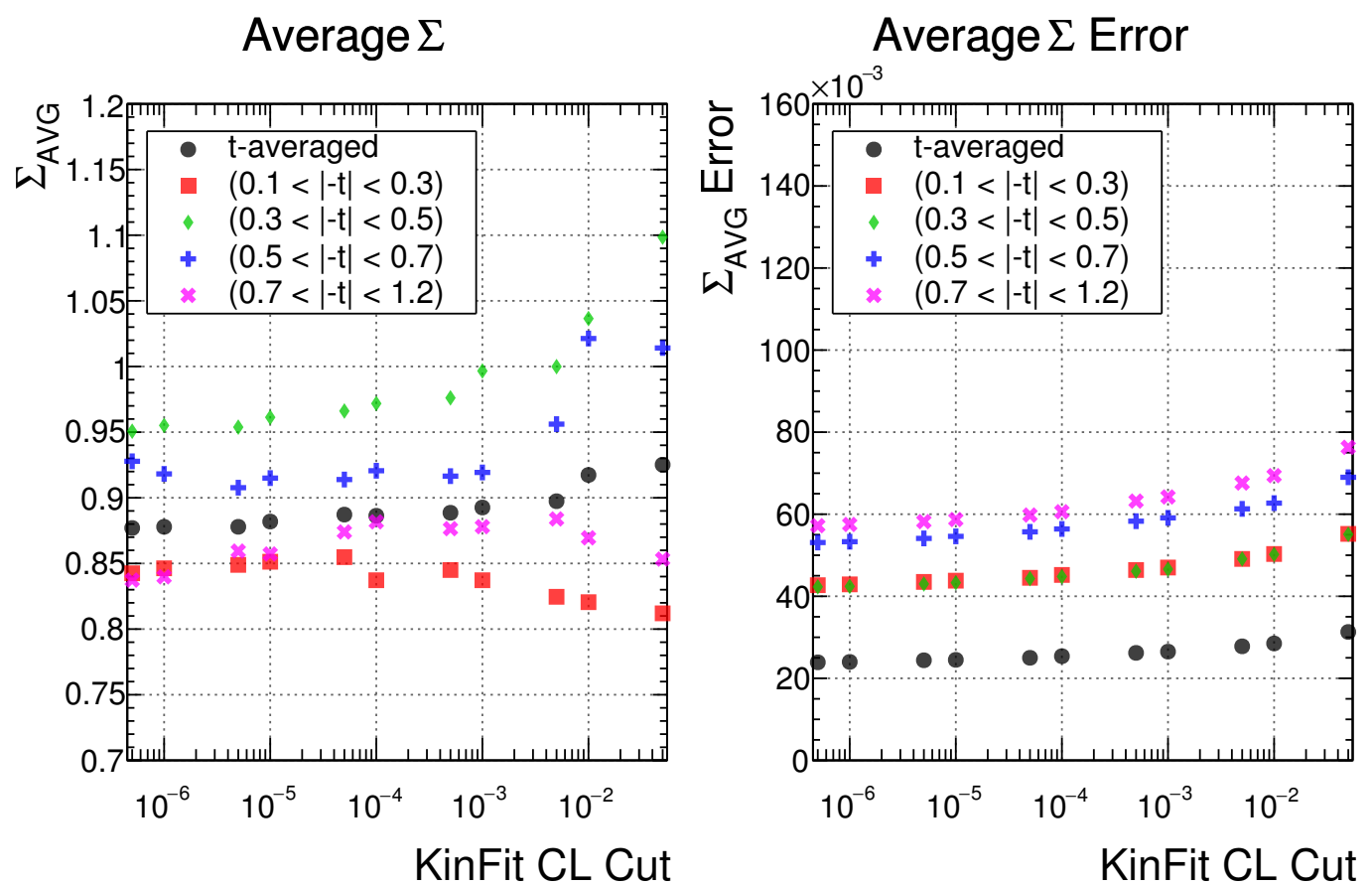

Figure 5.3: $\eta \rightarrow 3 \pi^{0}$ average SB-corrected $\Sigma$ asymmetries for the studied bins of $t$ versus kinematic fit confidence level cut (left) and statistical errors on those asymmetries (right) (colour online).

Fractional Background

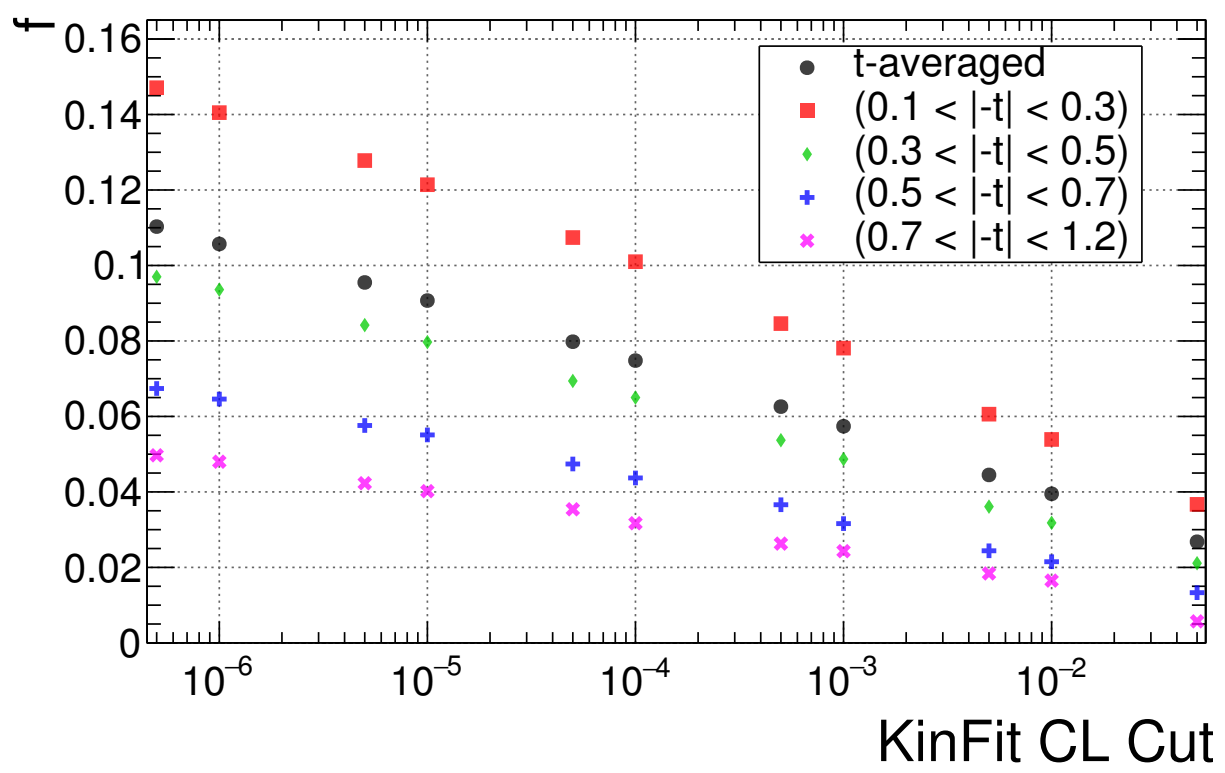

Figure 5.4: $\eta \rightarrow 2 \gamma$ dilution factor, $f$, for the studied bins of $t$ versus kinematic fit confidence level cut (colour online). 
The average SB-corrected $\Sigma$ asymmetry results for the $\eta \rightarrow 3 \pi^{0}$ are shown in Figure $5.3^{1}$, with the $0 / 90$ and $45 / 135$ SB-corrected asymmetries shown in Figures B.3 and B.4. The $\eta \rightarrow 2 \gamma$ results are shown in Figure 5.4 for $f$ and Figure 5.5 for the SB-corrected average $\Sigma$ asymmetry, with the $0 / 90$ and 45/135 results shown in Figures B.5 and B.6.
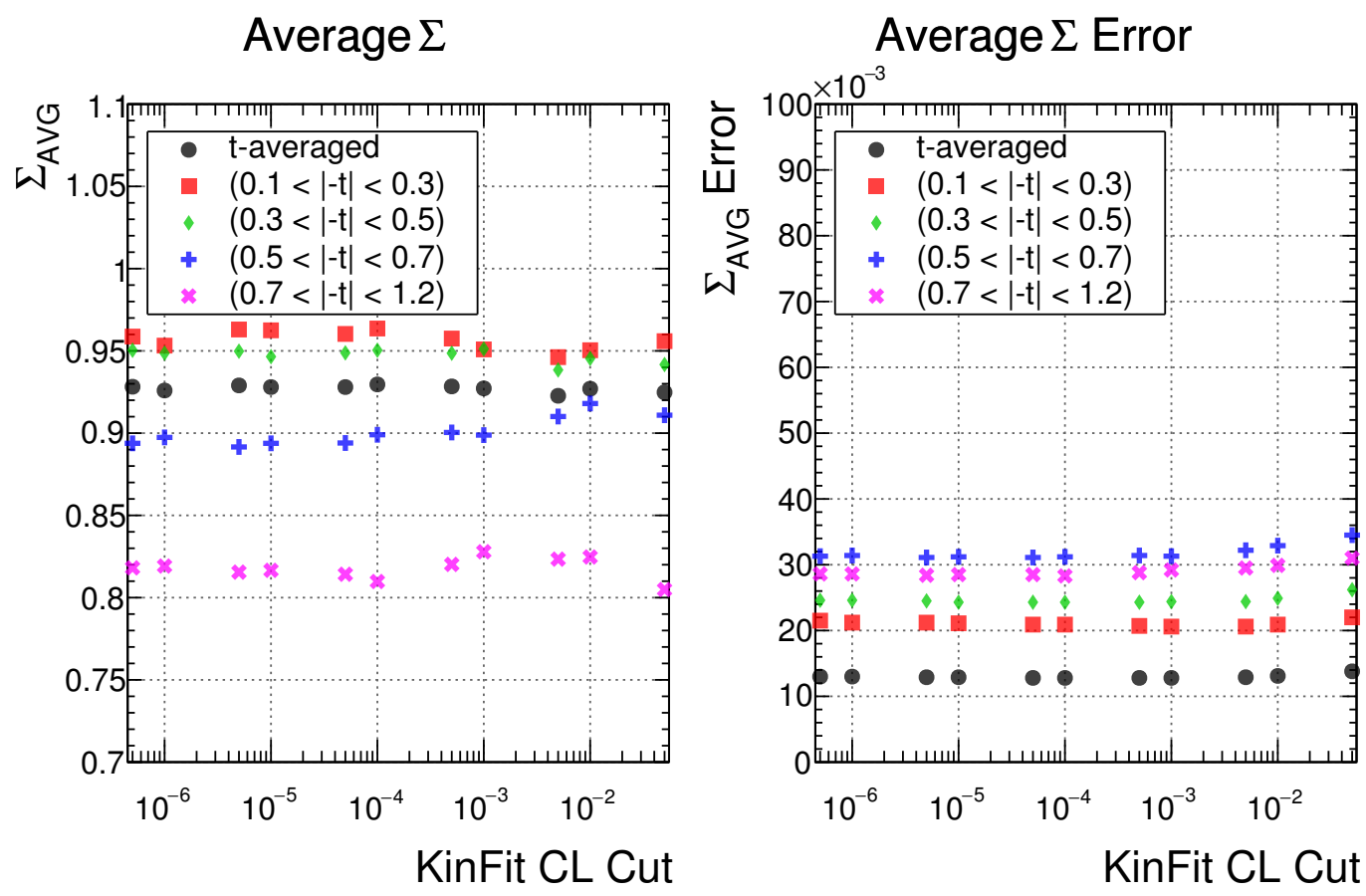

Figure 5.5: $\eta \rightarrow 2 \gamma$ average SB-corrected $\Sigma$ asymmetries for the studied bins of $t$ versus kinematic fit confidence level cut (left) and statistical errors on those asymmetries (right) (colour online).

Results for the $\eta^{\prime} \rightarrow \pi^{+} \pi^{-} \eta$ channel also feature the systematic error on $\Sigma_{\mathrm{SB}}$ versus CL cut. This value is the chosen systematic error (0.19, see Section 5.2.1) propagated through the statistical error formula (Equation 3.8), which depends on $f$, the dilution factor. As the CL cut increases, the fraction of background under the signal peak decreases, meaning the calculated systematic on the side-band asymmetry also decreases. The $\eta^{\prime}$ results for $f$ are shown in Figure 5.6, and the results for the SBcorrected average $\Sigma$ asymmetry (along with the systematic error on $\Sigma_{\mathrm{SB}}$ ) are shown

\footnotetext{
${ }^{1}$ Since there is no fitted background in this decay channel, there is no measure of $f$.
} 
in Figure 5.7. The lower-right plot in this figure shows the total error, the statistical and side-band systematic errors added in quadrature. The 0/90 and 45/135 results are shown in Figures B.7 and B.8.

\section{Fractional Background}

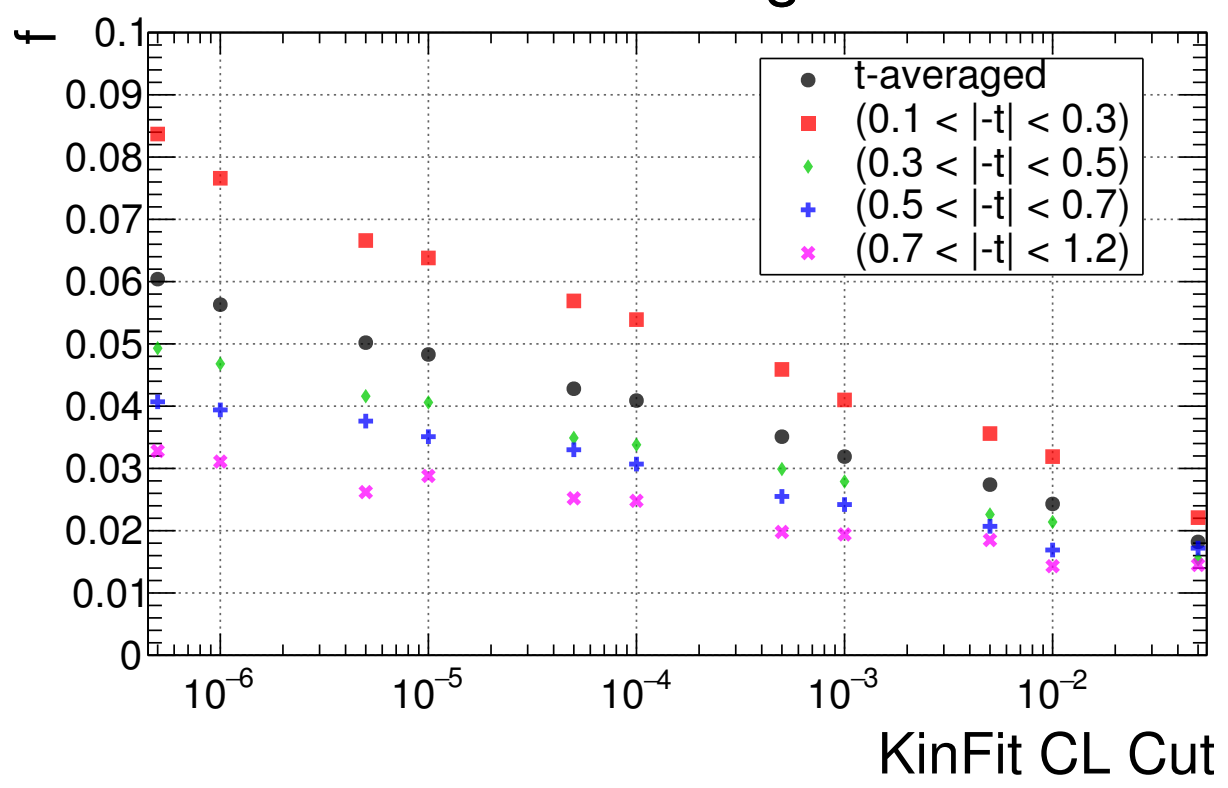

Figure 5.6: $\eta^{\prime} \rightarrow \pi^{+} \pi^{-} \eta$ dilution factor, $f$, for the studied bins of $t$ versus kinematic fit confidence level cut (colour online).

For channels with background, the dilution factor decreases monotonically with tighter CL cuts as events with higher kinematic fit $\chi^{2}$ are filtered out. At the tightest tested confidence level cuts, the amount of background under the peak is only a few percent, leaving a very pure signal and small background corrections. However, tight cuts also eliminate good signal events. Indeed, for all decay channels, the average asymmetries as a function of CL cut become less 'stable' at the tightest tested confidence level cuts (meaning the trends of average asymmetries versus CL cut become less flat with tighter cuts) and tend to be flat for a CL cut between $10^{-3}$ and $10^{-5}$. The statistical errors of the fits are very slowly rising with CL cut, growing more noticeably at the highest tested CL cuts. This is expected, since the number of signal events in the peak region steadily decreases with increasing CL cut. 

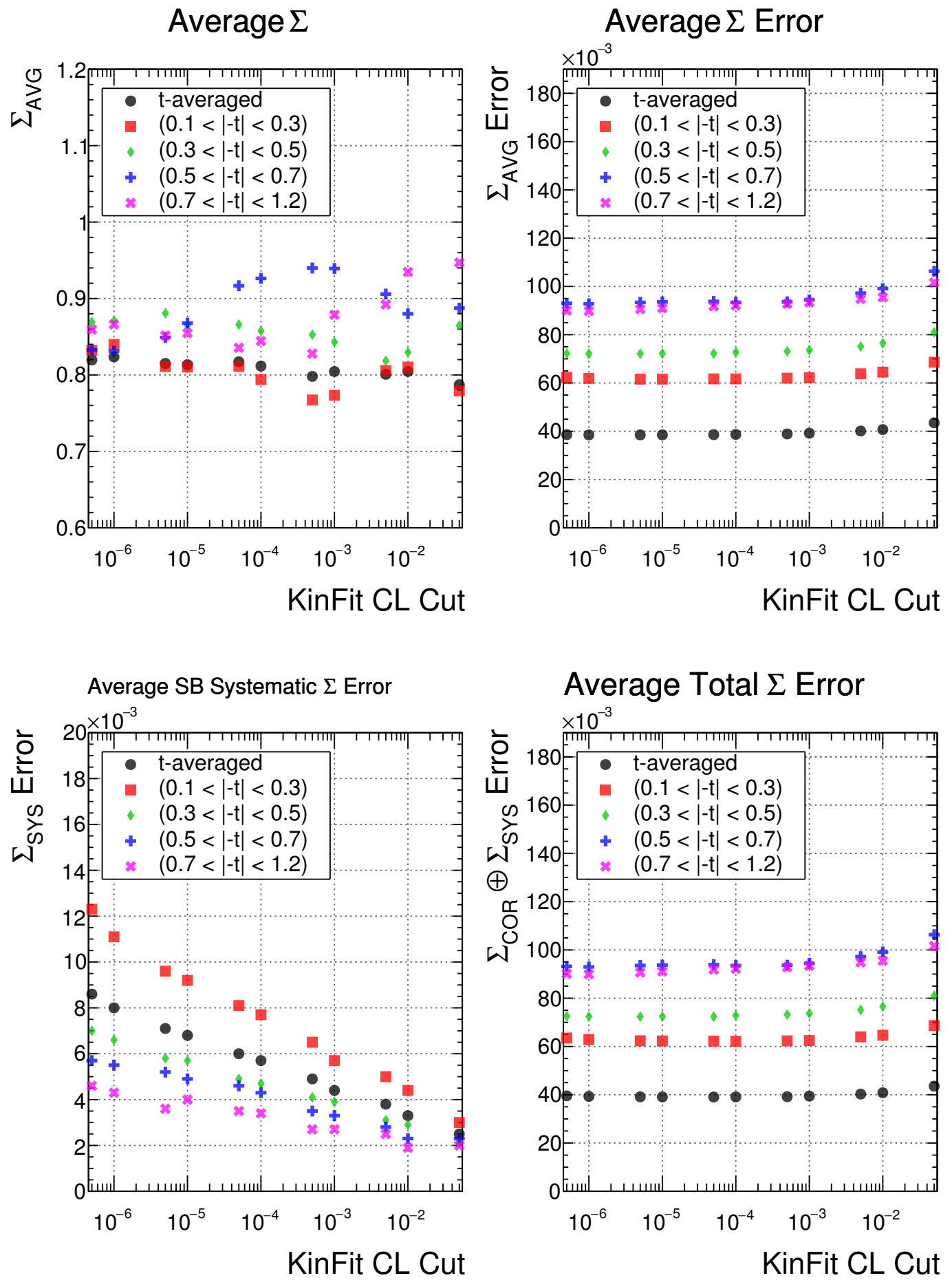

Figure 5.7: $\eta^{\prime} \rightarrow \pi^{+} \pi^{-} \eta$ average SB-corrected $\Sigma$ asymmetries for the studied bins of $t$ versus kinematic fit confidence level cut (top left), the statistical errors on those asymmetries (top right), the systematic uncertainties on those asymmetries to do with the choice of $\Sigma_{\mathrm{SB}}$ (bottom left), and the total error (quadratic sum of statistical and systematic, bottom right) (colour online). 
In essence, the results of this study are showing that the choice of CL cut does not significantly affect the final results or the errors on the final results so long as the chosen cut is not extremely tight or loose so as to cut out too many events or invite in too much background. As such, a cut in the stable range, $10^{-5}$, which keeps as much signal as possible is chosen for all decay channels.

\section{$5.2 \quad \eta^{\prime}$ Asymmetry Systematic Studies}

In order to estimate the systematic effects of the event selection and analysis methods on the asymmetry results, a number of systematic studies are done on each stage of the analysis. Each major selection cut is varied, the fit function parameters $\left(\phi_{0}, F_{R}\right)$ are varied, the functional form is tested, the $\eta^{\prime} \mathrm{SB}$ asymmetry is investigated, and the instrumental asymmetry is checked. In each case, the full analysis is done with the altered parameter, cut value, fit function, etc. and the resulting asymmetries are compared to the nominal parameter, value, fit function, etc. The systematic uncertainties assigned to the $\eta^{\prime}$ asymmetry as a result of each of these variations is discussed in the corresponding sections to follow.

The analysis of systematic uncertainties is carried out only for the $\eta^{\prime}$ decay channel. The focus of this thesis is on the new physics results of the $\eta^{\prime}$ beam asymmetry at GlueX energies, and the analyses of the three $\eta$ channels are maintained primarily to validate the $\eta^{\prime}$ analysis methodology. Results from the three $\eta$ decay channels that agree with one another, and that agree with past results, lend confidence to the analysis methodology used for the $\eta^{\prime}$ analysis. A similar systematic uncertainty study is being carried out for these three $\eta$ decay channels by another graduate student within the GlueX Collaboration. For these reasons, the decision was made to focus on analysis of systematic uncertainties only for the $\eta^{\prime}$ result. 


\subsubsection{Side-Band Asymmetry}

Since the nature of the contents of the $\eta^{\prime}$ side-band region (depicted in Figure 4.24) are not entirely known, it is likely that the side-band asymmetry for this channel is mass-dependent. The asymmetry analysis is carried out multiple times using different ranges in mass for the side-band region. The extracted side-band asymmetry is then plotted versus the central values of the mass ranges used. The idea is to fit these few data points and extrapolate down to the $\eta^{\prime}$ mass to estimate $\Sigma_{\mathrm{SB}}$ underneath the signal peak in the case that the asymmetry varies with mass.

Because there are generally low statistics in this channel, and because the mass side-band region is being split up into multiple bins, the fits to the asymmetry are not perfect. As such, multiple binnings are investigated along with multiple fit forms (constant, linear, and exponential). The goal is to find a reasonable systematic error in lieu of an accurate estimation of $\Sigma_{\mathrm{SB}}$ under the signal peak. For each form, a fit is performed and extrapolated down to the $\eta^{\prime}$ mass $(0.958 \mathrm{GeV})$. The range in extrapolated asymmetries from the three fits gives some evaluation of the possible values of the side-band asymmetry under the peak and is used as an estimate of the systematic uncertainty on $\Sigma_{\mathrm{SB}}$. With the limited statistics, only the $t$-averaged results are investigated, and the systematic uncertainty extracted is applied evenly to all $t$ bins.

Figure 5.8 shows the $\eta^{\prime} \rightarrow \pi^{+} \pi^{-} \eta$ side-band asymmetries (using the $45 / 135$ data set) as found using various mass regions (depicted as horizontal error bars) along with the constant, linear, and exponential fits. The range of extrapolated $\Sigma_{\mathrm{SB}}$ values under the signal peak based on the three fits is displayed on the plot.

The data shows a sloping trend, implying that the side-band asymmetry is massdependent and that the background asymmetry under the peak is not necessarily equal to the asymmetry in the nominal side-band region. The three fits to the data, extrapolated down to the $\eta^{\prime}$ mass, give a spread of 0.19 , which is the systematic 
SB $\Sigma$ vs. SB Mass Range Median (t-Averaged)

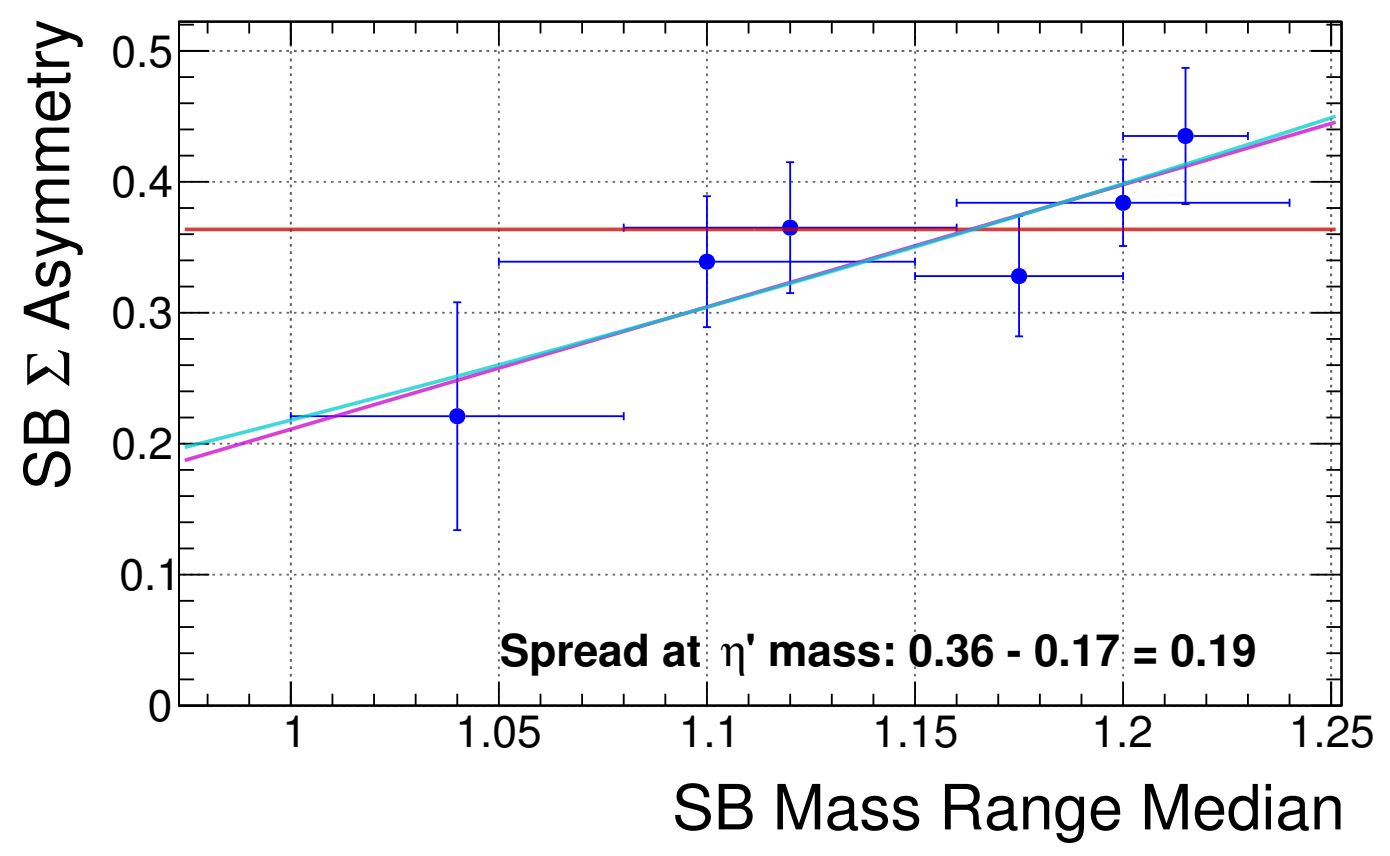

Figure 5.8: Resultant 45/135 $\Sigma_{S B}$ for various mass bins with a constant (red), linear (magenta), and exponential (cyan) fit. A range of values from extrapolations of the fits down to $0.958 \mathrm{GeV}$ is displayed (colour online).

error assigned to $\Sigma_{\mathrm{SB}}$. This error is propagated through the second error term in Equation 3.8 (in place of $\delta_{\Sigma_{\mathrm{SB}}}$ ) to give $\delta_{\mathrm{SBSYS}}$, as in Equation 5.1. Table 5.1 shows the systematic error on each $t$ bin on the average $\Sigma$ asymmetry calculated using this equation.

$$
\delta_{\mathrm{SBSYS}}=\sqrt{\left(\frac{0.19 * f}{1-f}\right)^{2}}
$$

\begin{tabular}{|c||c|}
\hline$t$ Range $\left(\mathrm{GeV}^{2}\right)$ & Systematic Uncertainty $\left(\delta_{\text {SBSYS }}\right)$ \\
\hline \hline $0.1<-t<0.3$ & 0.0219 \\
\hline $0.3<-t<0.5$ & 0.0113 \\
\hline $0.5<-t<0.7$ & 0.0076 \\
\hline $0.7<-t<1.2$ & 0.0089 \\
\hline \hline
\end{tabular}

Table 5.1: Systematic uncertainties for each bin of $t$ as found by Equation 5.1 on the average asymmetry. 


\subsubsection{1 $\eta$ Side-Band Asymmetry}

A similar study was carried out for the two $\eta$ decay channels with background contributions to check for any mass dependency in the side-band asymmetry. Since the background in both these channels are predominantly from the $\omega$ meson, the assumption is that the asymmetry is not mass dependent since each mass bin should contain almost exclusively the same type of particle. Figures 5.9 and 5.10 show the side-band asymmetries (using the 0/90 data set) as found using various mass regions (depicted as horizontal error bars) for three ranges in $t$. There are significantly more statistics in these channels' side-band regions than for the $\eta^{\prime}$ decay channel, so the fits to the yield asymmetries are more stable with smaller errors in the $\eta$ channels. For both decay channels, in all $t$ ranges, there is no clear side-band asymmetry trends versus mass.

\section{3piq SB $\Sigma$ vs. SB Mass Range Median}

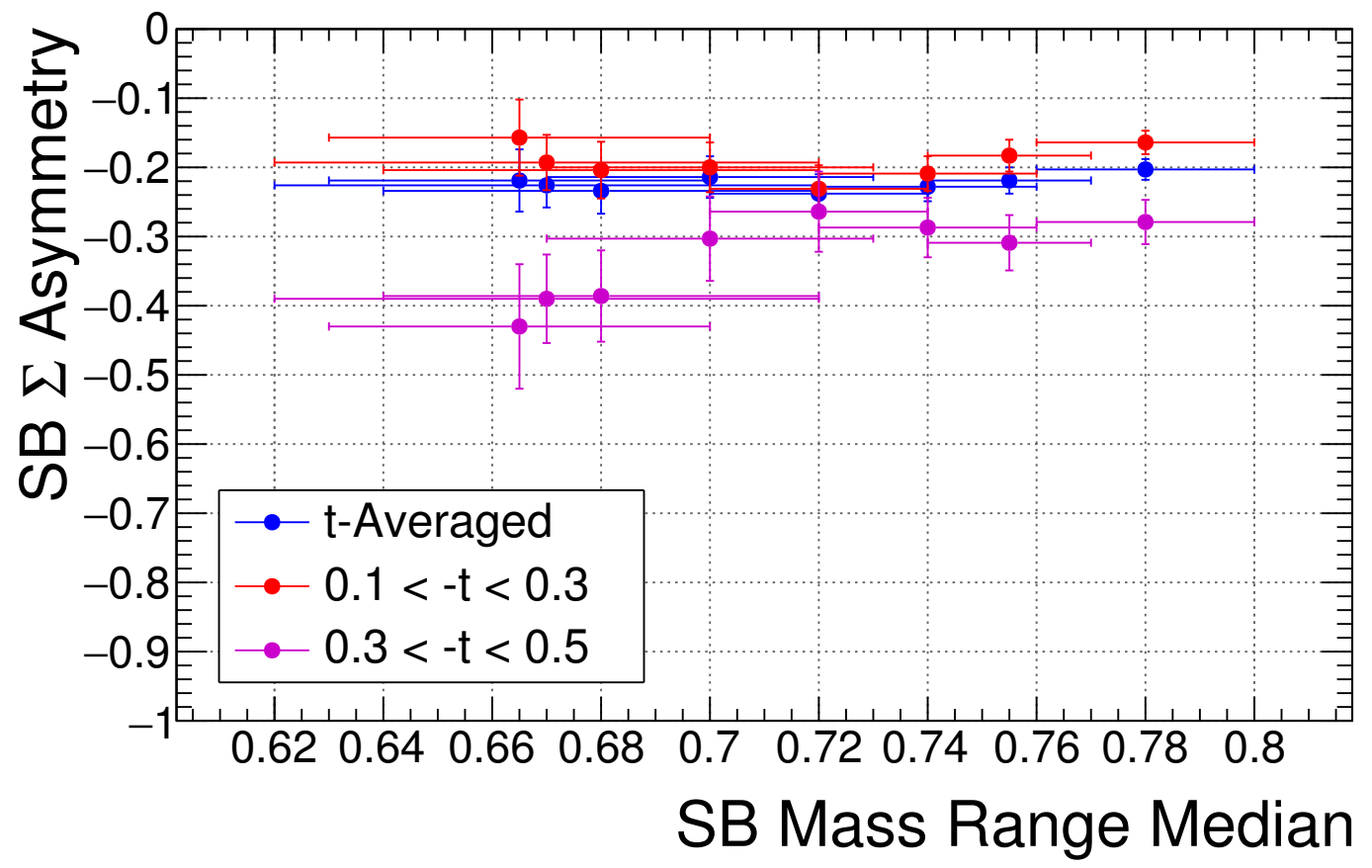

Figure 5.9: Resultant 0/90 $\eta \rightarrow \pi^{+} \pi^{-} \pi^{0} \Sigma_{S B}$ for various mass bins in three ranges of $t$ (see legend in plot) (colour online).

The lowest-tested mass range in the 3piq decay channel may show some deviation 


\section{$2 \mathrm{~g}$ SB $\Sigma$ vs. SB Mass Range Median}

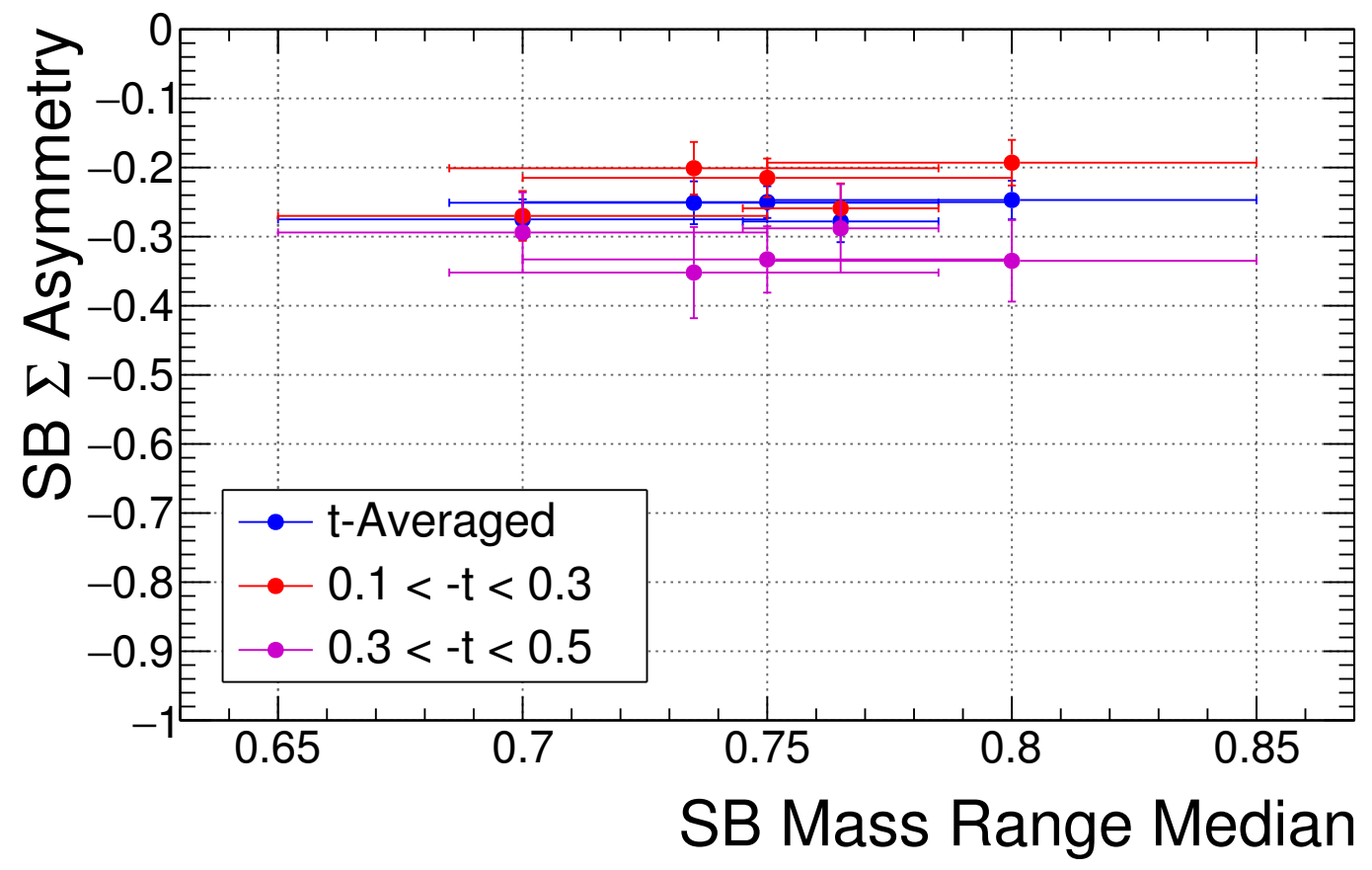

Figure 5.10: Resultant $0 / 90 \eta \rightarrow 2 \gamma \Sigma_{S B}$ for various mass bins in three ranges of $t$ (see legend in plot) (colour online).

in some $t$ ranges, but because of the lower statistics (and larger errors) for these data points, the trend is not statistically significant. For this reason, the assumptions that the side-band asymmetries in the nominal side-band regions for each analysis are equal to the asymmetries of the background under the signal peaks are maintained, and no corrections or errors are assigned in the $\eta$ channels to do with the side-band asymmetry corrections.

\subsubsection{Cut Variations}

For each major event selection cut, the asymmetry analysis is performed while varying the nominal cut to a looser and tighter version to test the systematic effects of the cuts on the final extracted results. Asymmetries are extracted in each $t$ bin for each variation. The nominal, looser, and tighter cut values used are given in Table 5.2. 


\begin{tabular}{|c||c|c|c|}
\hline Cut Variable & Looser Cut & Nominal Cut & Tighter Cut \\
\hline \hline Vertex Z $(\mathrm{cm})$ & $50<\mathrm{z}<80$ & $52<\mathrm{z}<78$ & $54<\mathrm{z}<76$ \\
\hline Vertex R $(\mathrm{cm})$ & $\mathrm{r}<1.5$ & $\mathrm{r}<1.0$ & $\mathrm{r}<0.5$ \\
\hline Fiducial BCAL $(\mathrm{cm})$ & $\mathrm{z}_{\gamma}<390$ & $\mathrm{z}_{\gamma}<380$ & $\mathrm{z}_{\gamma}<370$ \\
\hline Fiducial FCAL $(\mathrm{cm})$ & $20>\mathrm{r}_{\gamma}>105$ & $25>\mathrm{r}_{\gamma}>100$ & $30>\mathrm{r}_{\gamma}>95$ \\
\hline Particle $\Delta \mathrm{t}(\mathrm{ns})$ & $|\Delta \mathrm{t}|<6$ & $|\Delta \mathrm{t}|<4$ & $|\Delta \mathrm{t}|<2$ \\
\hline Proton P $(\mathrm{GeV})$ & $\mathrm{P}_{p}>0.30$ & $\mathrm{P}_{p}>0.25$ & $\mathrm{P}_{p}>0.20$ \\
\hline$\eta \rightarrow 2 \gamma$ Mass $(\mathrm{GeV})$ & $0.44<m_{2 \gamma}<0.64$ & $0.46<m_{2 \gamma}<0.62$ & $0.48<m_{2 \gamma}<0.60$ \\
\hline$\eta^{\prime}$ Mass Range & $\pm 2.2 \sigma$ & $\pm 2 \sigma$ & $\pm 1.8 \sigma$ \\
\hline KinFit CL & $\mathrm{CL}>10^{-6}$ & $\mathrm{CL}>10^{-5}$ & $\mathrm{CL}>10^{-4}$ \\
\hline \multicolumn{4}{|r}{}
\end{tabular}

Table 5.2: Nominal, tighter, and looser cut variations for the study of systematic sensitivity of the $\Sigma$ asymmetry to event selection cuts.

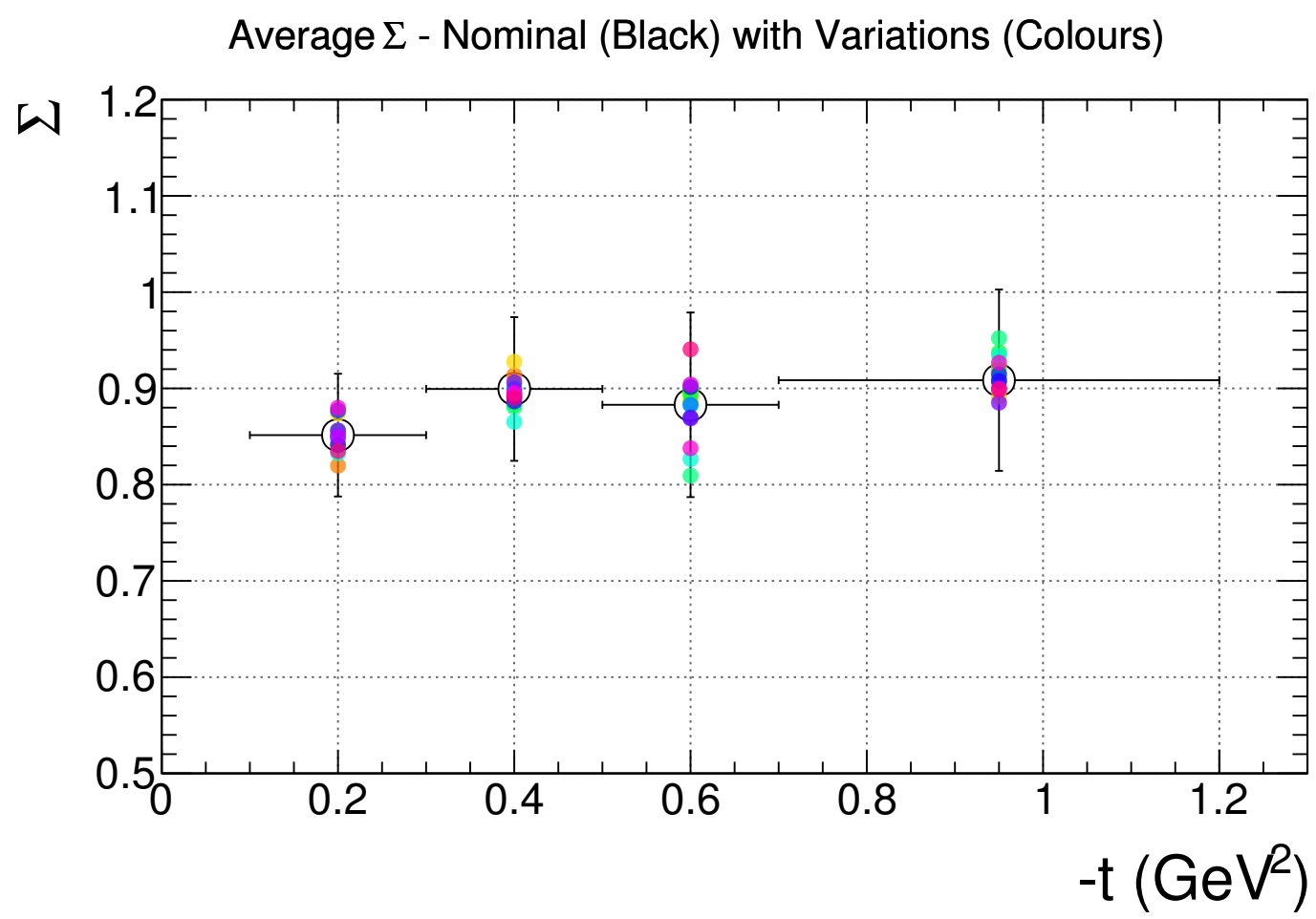

Figure 5.11: Average $\eta^{\prime}$ SB-corrected asymmetries for nominal (black open circles with error bars) and varied (coloured filled circles) cuts. Colours are as in the legend shown in Figure 5.12 (colour online).

Figure 5.11 shows the average SB-corrected asymmetry binned in $t$ for the nominal cuts (black open circles with error bars) and for the cut variations (various coloured circles with no error bars). Each colour in the plot represents a different cut variation, with each colour's corresponding variation given in Figure 5.12. Figures B.9 and B.10 
in Appendix B show the nominal and variational asymmetries for the $0 / 90$ and 45/135 data sets respectively.

\begin{tabular}{|lll|}
\hline Nominal Cuts & \\
Vertex Z (Looser) & Vertex Z (Tighter) \\
Vertex R (Looser) & Vertex R (Tighter) \\
Fiducial BCAL (Looser) & Fiducial BCAL (Tighter) \\
Fiducial FCAL (Looser) & Fiducial FCAL (Tighter) \\
Proton P (Looser) & Proton P (Tighter) \\
Eta $->$ 2g Mass (Looser) & Eta -> 2g Mass (Tighter) \\
EtaPrime Mass Range (Looser) & $\bullet$ & EtaPrime Mass Range (Tighter) \\
KinFit FOM (Looser) & KinFit FOM (Tighter) \\
\hline
\end{tabular}

Figure 5.12: Legend for cut systematics plots (Figures 5.11, B.9, and B.10) and instrumental asymmetry plots (Figures 5.13, B.11, B.12, and B.13) (colour online).

Each cut variation falls within the statistical error bar of the nominal cuts, meaning that the analysis is fairly stable with changes in event selection cuts. A systematic error, $\delta_{\mathrm{CUTSYS}}$, is evaluated for each bin by finding a squared sum of the differences between the cut-varied average asymmetries and nominal average asymmetries in a manner similar to a standard deviation, as in Equation 5.2.

$$
\delta_{\mathrm{CUTSYS}}=\sqrt{\frac{\sum_{n=1}^{N_{\mathrm{VAR}}}\left(\sum_{\mathrm{VAR}, n}-\Sigma_{\mathrm{NOM}}\right)^{2}}{N_{\mathrm{VAR}}-1}}
$$

Here, $\Sigma_{\mathrm{NOM}}$ is the nominal asymmetry for a particular bin, $\Sigma_{\mathrm{VAR}, n}$ is the cut-varied asymmetry for the same bin with cut variation $n$, and $N_{\mathrm{VAR}}$ is the number of total variations. Table 5.3 gives the calculated systematic uncertainties on the nominal average asymmetries using this equation. 


\begin{tabular}{|c||c|}
\hline$t$ Range $\left(\mathrm{GeV}^{2}\right)$ & Systematic Uncertainty $\left(\delta_{\text {CUTSYS }}\right)$ \\
\hline \hline $0.1<-t<0.3$ & 0.0161 \\
\hline $0.3<-t<0.5$ & 0.0149 \\
\hline $0.5<-t<0.7$ & 0.0328 \\
\hline $0.7<-t<1.2$ & 0.0186 \\
\hline
\end{tabular}

Table 5.3: Systematic uncertainties for each bin of $t$ as found by Equation 5.2 on the average asymmetry.

\subsubsection{Instrumental Asymmetry}

As detailed in Section 3.2, forming the yield asymmetry between PARA and PERP diamond orientations cancels out the $\phi$-dependent acceptance, $A(\phi)$. However, if some other instrumental asymmetry exists, it can be seen in the direct sum of the two orientations' $\phi$-dependent yields. Fits to the sum of the perpendicular orientations are done for peak region and side-band region data for both the 0/90 and 45/135 data sets in every $t$ bin and for every cut variation to extract the instrumental asymmetry. The fit is done with the function shown in Equation 5.3.

$$
f_{\text {inst }}(\phi)=C\left(1+\Sigma_{\text {inst }} \cos \left(2\left(\phi-\phi_{0}\right)\right)\right)
$$

Here, $\phi_{0}$ is held constant at the same value as for the asymmetry fits, $C$ is an overall normalization factor, and $\Sigma_{\text {inst }}$ is the instrumental asymmetry. If all instrumental asymmetries and acceptance effects are cancelled by forming the asymmetry between PARA and PERP orientation data, the fits here should give a value of zero for $\Sigma_{\text {inst }}$. Figure 5.13 shows $\Sigma_{\text {inst }}$ in bins of $t$ for the $0 / 90$ data set (peak region), again with black open circles denoting the nominal cuts and coloured circles denoting cut variations (colours are the same as given in Figure 5.12). The plot also features a fit to the nominal data points to check the consistency of the instrumental asymmetries with 0. Figure B.11 shows the same for the 0/90 side-band region, and Figures B.12 and B.13 show the peak region and side-band region for the 45/135 data set, respectively. 


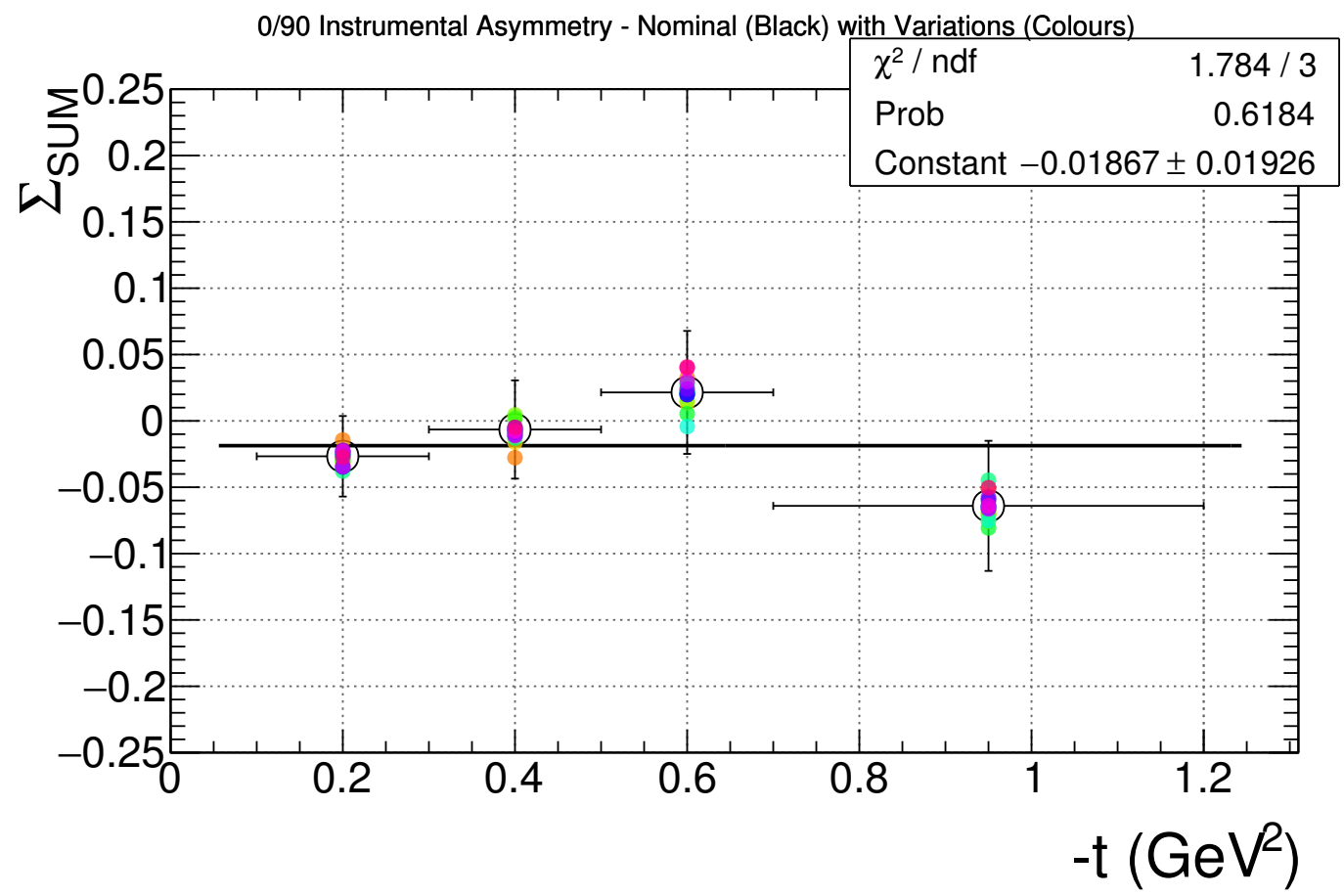

Figure 5.13: $0 / 90 \eta^{\prime}$ peak region asymmetries for nominal (black open circles with error bars) and varied (coloured filled circles) cuts. Colours are as in the legend shown in Figure 5.12 (colour online).

The instrumental asymmetries are relatively stable with varied cuts and are clustered about 0 for each orientation and mass region. The results of the constant function fits to the nominal instrumental asymmetries are given in Table 5.4. There is no strong indication of a residual instrumental asymmetry, and no additional systematic uncertainty on the $\eta^{\prime}$ beam asymmetry is assigned.

\begin{tabular}{|c||c|}
\hline Data Set: Mass Region & Constant Fit Parameter \\
\hline \hline 0/90: Peak Region & $-0.0 .187 \pm 0.0193$ \\
\hline 0/90: Side-Band Region & $0.0104 \pm 0.0118$ \\
\hline 45/135: Peak Region & $-0.0082 \pm 0.0199$ \\
\hline 45/135: Side-Band Region & $-0.0131 \pm 0.0123$ \\
\hline
\end{tabular}

Table 5.4: Results of constant function fits to the nominal instrumental asymmetries for each data set and mass region. The fit parameter is consistent with 0 in each case. 


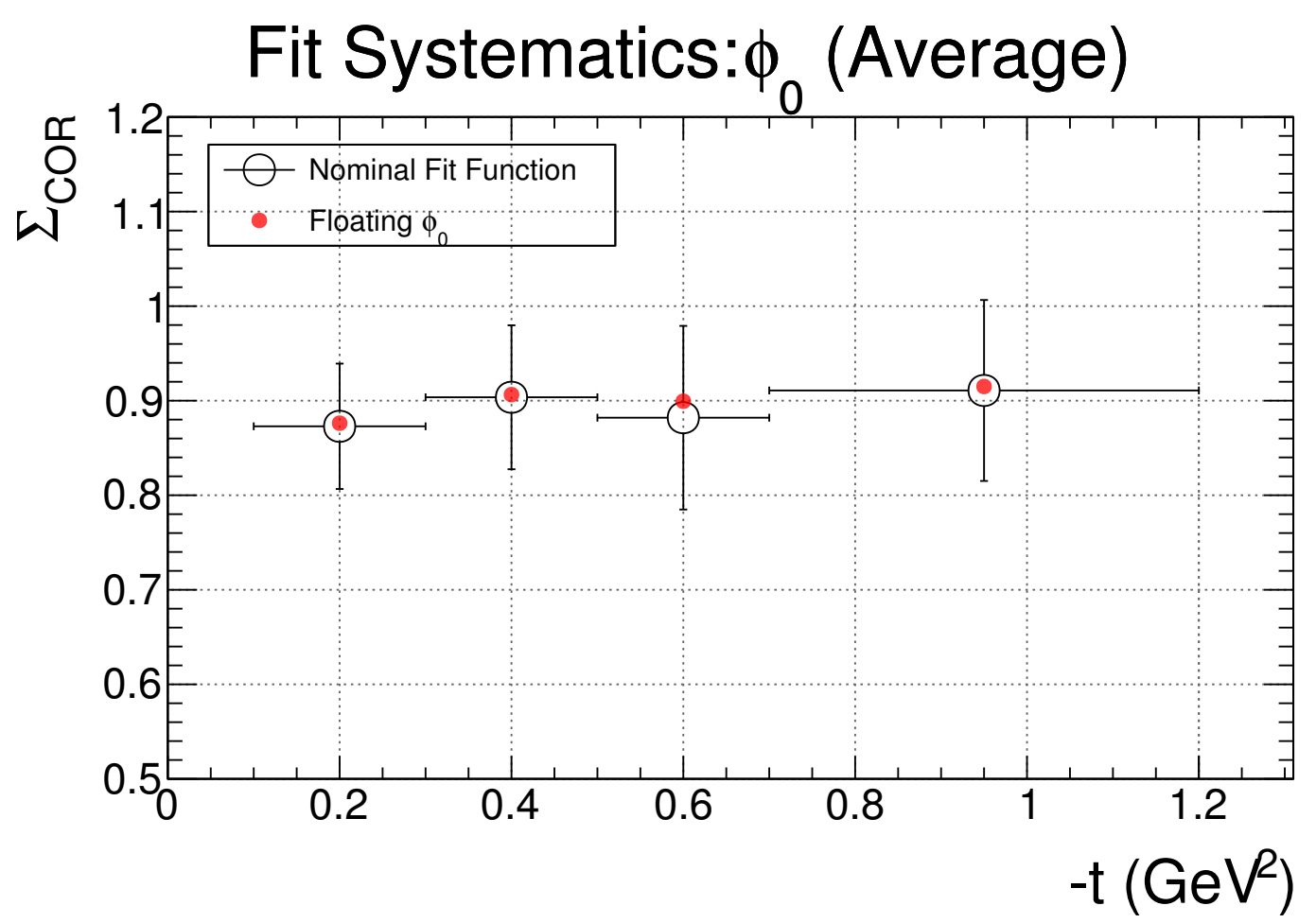

Figure 5.14: Average SB-corrected $\eta^{\prime}$ asymmetries for nominal fit parameters (black open circles with error bars) and floating $\phi_{0}$ fit (red filled circles).

\subsubsection{Phase Offset}

The phase offset, $\phi_{0}$, in the asymmetry fit function (Equation 3.7) is fixed from a study of the high-statistics ( $t$-averaged) $\eta \rightarrow 2 \gamma$ channel for the $0 / 90$ and 45/135 data sets separately, as discussed in Section 3.2.2. These should be universal constants in the analyses, since the offset cannot depend physically on the four-momentum transfer squared or decay channel. However, to test the sensitivity to differences in this fit parameter, the asymmetry analyses are performed again using the nominal cuts and fit parameters but allowing $\phi_{0}$ to be a free parameter. The final average asymmetry results for this case (red filled circles) and the nominal case (black open circles) are shown in Figure 5.14. A histogram of the difference between the free-parameter asymmetries and the nominal asymmetries over all bins is shown in Figure 5.15. This provides a measurement of the spread of asymmetries induced by letting the phase 
offset vary. Based on the standard deviation of this distribution, a systematic error of $0.6 \%$ is assigned to account for any sensitivities to the choice of phase offset in the fitted asymmetries.

\section{Fit Systematics: $\phi_{0}$ (Variation - Nominal)}

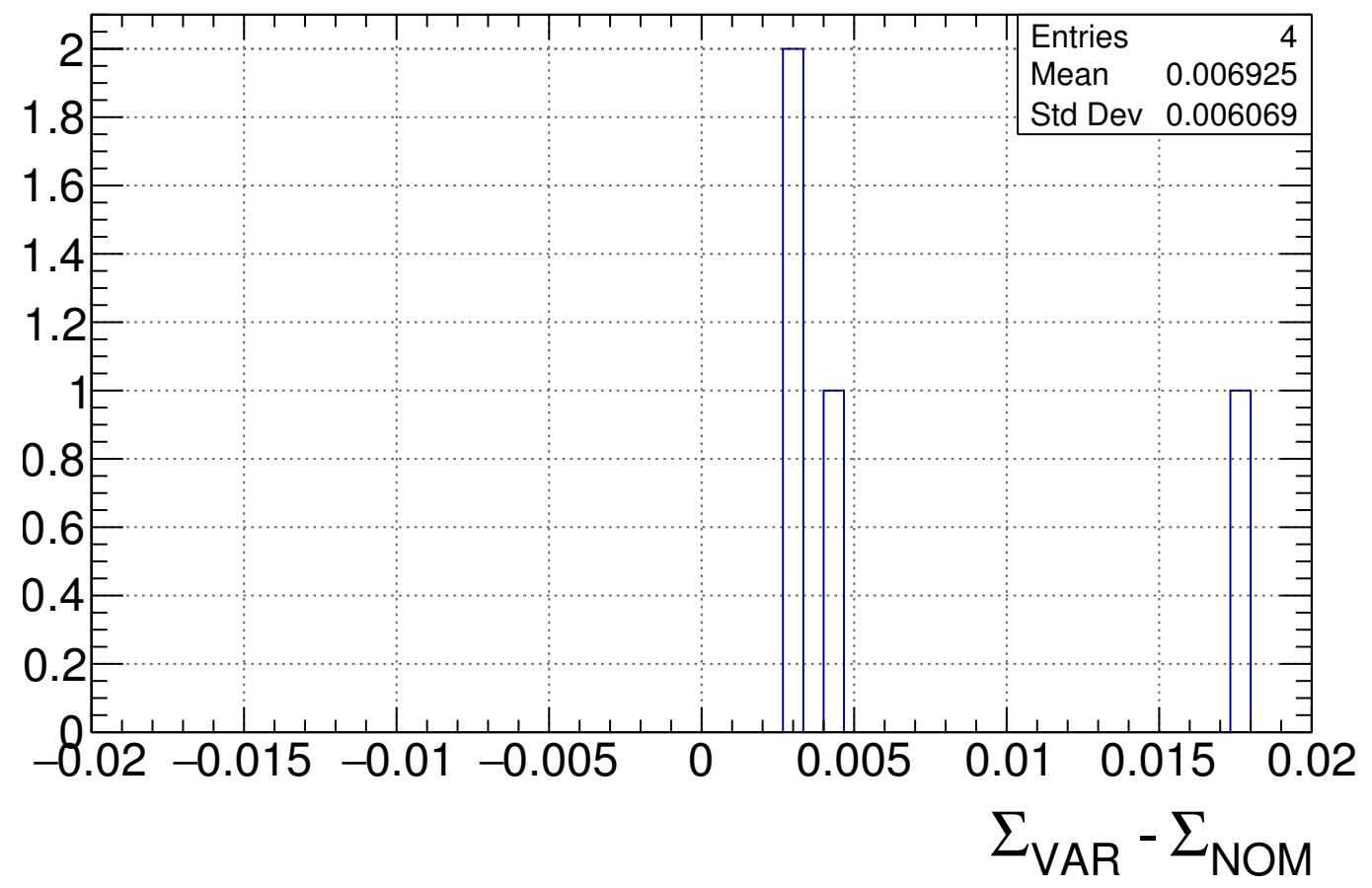

Figure 5.15: Histogram of differences between floating $\phi_{0}$ fit asymmetries and nominal fit asymmetries presented in Figure 5.14. The width of this distribution guides a selection of systematic uncertainty applied to the average asymmetry due to the choice of $\phi_{0}$.

\subsubsection{Flux Ratio}

The flux ratio, $F_{R}$, is the ratio of PARA to PERP PS triggers for a given data set. It is used to scale the PARA data so that the proper asymmetry can be formed to cancel out $\phi$-dependant acceptance effects. The sensitivity of the asymmetry analysis to the flux ratio is evaluated by repeating the analysis with slightly higher and lower values for the flux ratio in the same way as the cut variation systematic study. An estimate of the systematic deviation of the PS yields, coming from the efficiency of 


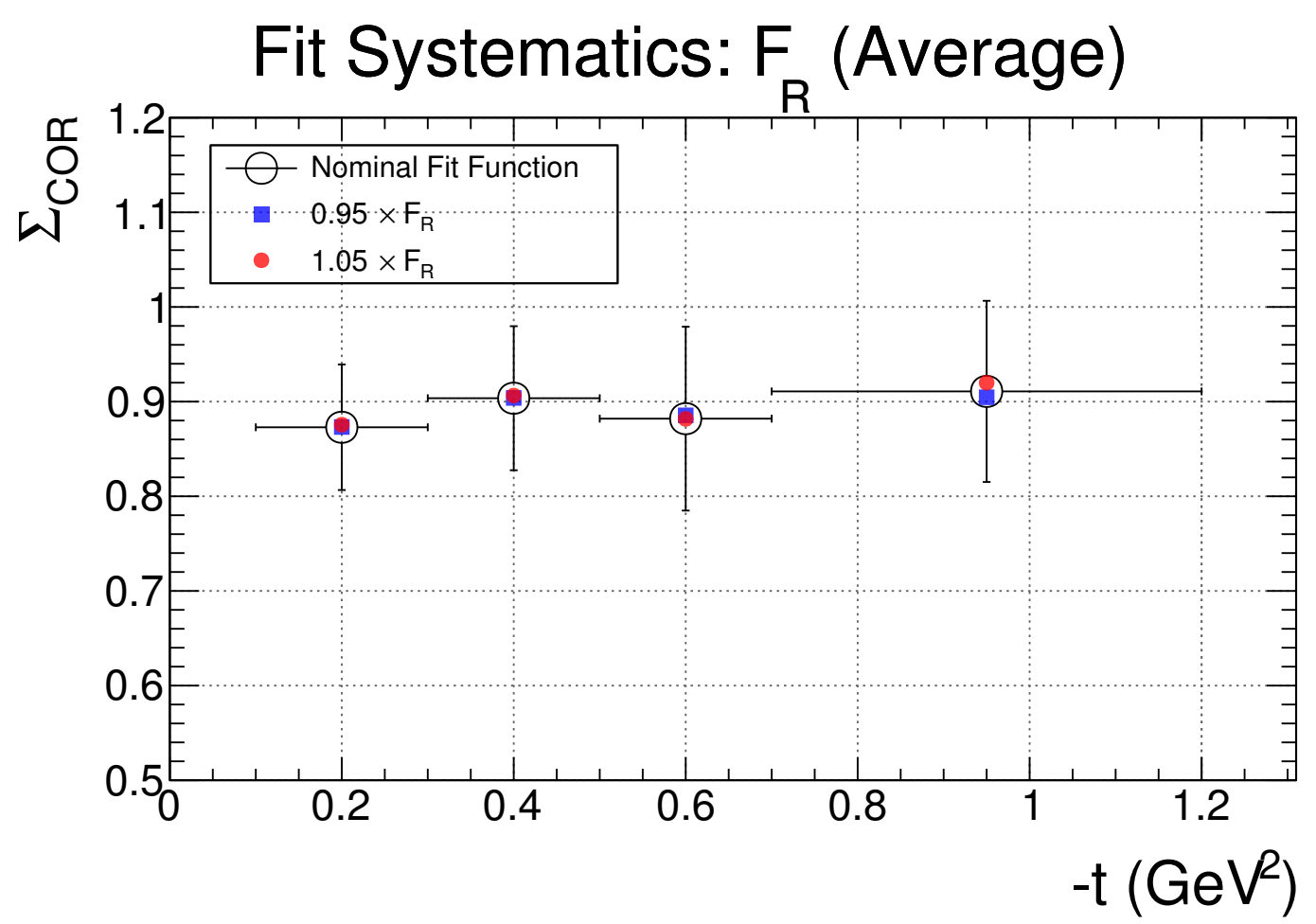

Figure 5.16: Average SB-corrected $\eta^{\prime}$ asymmetries for nominal fit parameters (black open circles with error bars), a 5\% increase in both 0/90 and 45/135 flux ratios (red filled circles), and a $5 \%$ decrease in those flux ratios (blue squares) (colour online).

the PS, is $\pm 5 \%$ [112]. As such, Figure 5.16 shows the average asymmetry in bins of $t$ using 0/90 and 45/135 flux ratio values that are 5\% higher (red filled circles), 5\% lower (blue squares), and nominal (black open circles). A histogram of the difference between the $F_{R}$-varied asymmetries and the nominal asymmetries over all bins is shown in Figure 5.17. The standard deviation of this distribution suggests a systematic uncertainty on the average asymmetries to do with the flux ratio determination of $0.4 \%$.

\subsubsection{Fit Function Variations}

The fit function used to extract $\Sigma$, Equation 3.7, assumes that the yield asymmetry constructed from PERP and PARA $\phi$-dependent yields is a sinusoidal function about $\Sigma=0$. However, if the flux ratio, $F_{R}$, is systematically incorrect, the PERP and 
Fit Systematics: $F_{R}$ (Variation - Nominal)

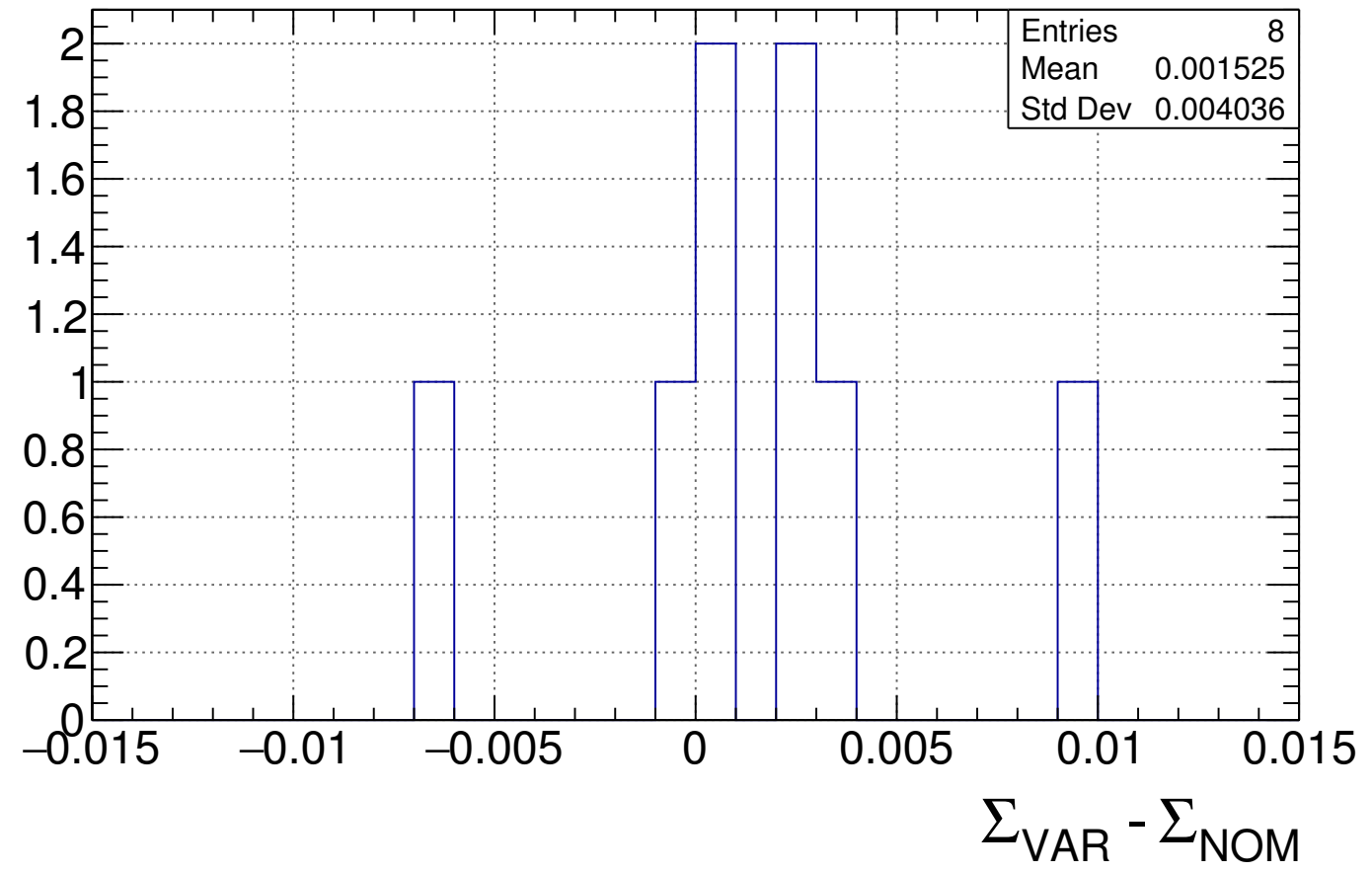

Figure 5.17: Histogram of differences between varied $F_{R}$ fit asymmetries and nominal fit asymmetries presented in Figure 5.16. The width of this distribution guides a selection of systematic uncertainty applied to the average asymmetry due to the PS determination of $F_{R}$ for both data sets (colour online).

PARA yield distributions would not be scaled properly, and when the yield asymmetry is constructed, it would be shifted from 0 . As a check on the flux ratio, the analysis is repeated with an additional constant term in the fit function, as in Equation 5.4.

$$
f(\phi)=\frac{\left(P_{\perp}+P_{\|}\right) \Sigma \cos \left(2\left(\phi-\phi_{0}\right)\right)}{2+\left(P_{\perp}-P_{\|}\right) \Sigma \cos \left(2\left(\phi-\phi_{0}\right)\right)}+A
$$

Figure 5.18 shows the average asymmetry in bins of $t$ for the nominal fit function (black open circles) and the altered fit function (red filled circles). No significant differences are seen. Figure 5.19 shows the fitted additional parameter, $A$, in each bin of $t$ for the $0 / 90$ and 45/135 data sets and for the peak and side-band regions. Fits to constant functions in the latter plot indicate no systematic deviation from 0 , implying that the flux ratios determined by the PS measurements are reasonable. 
Fit Systematics: Fit + Constant (Average)

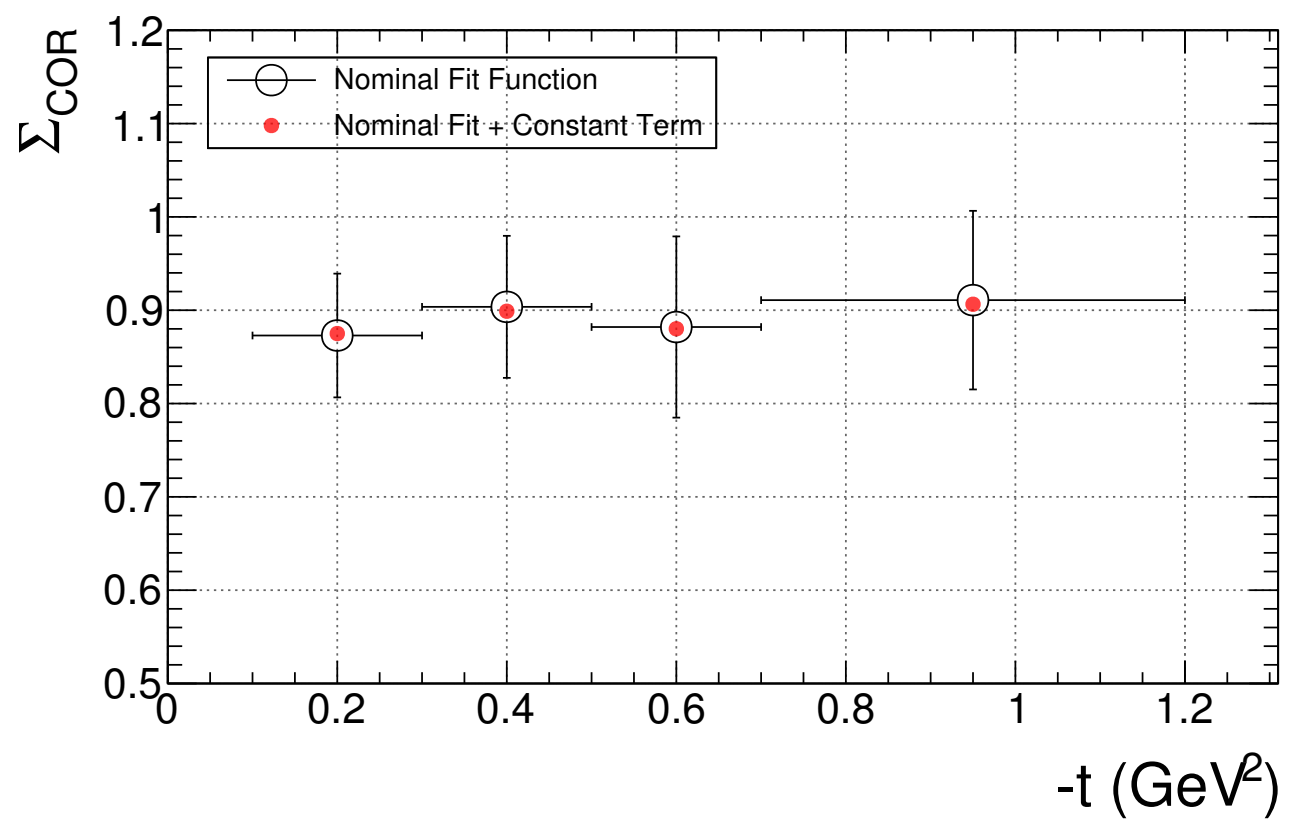

Figure 5.18: Average SB-corrected $\eta^{\prime}$ asymmetries for the nominal (black open circles with error bars) and altered (red filled circles) fit function (colour online).

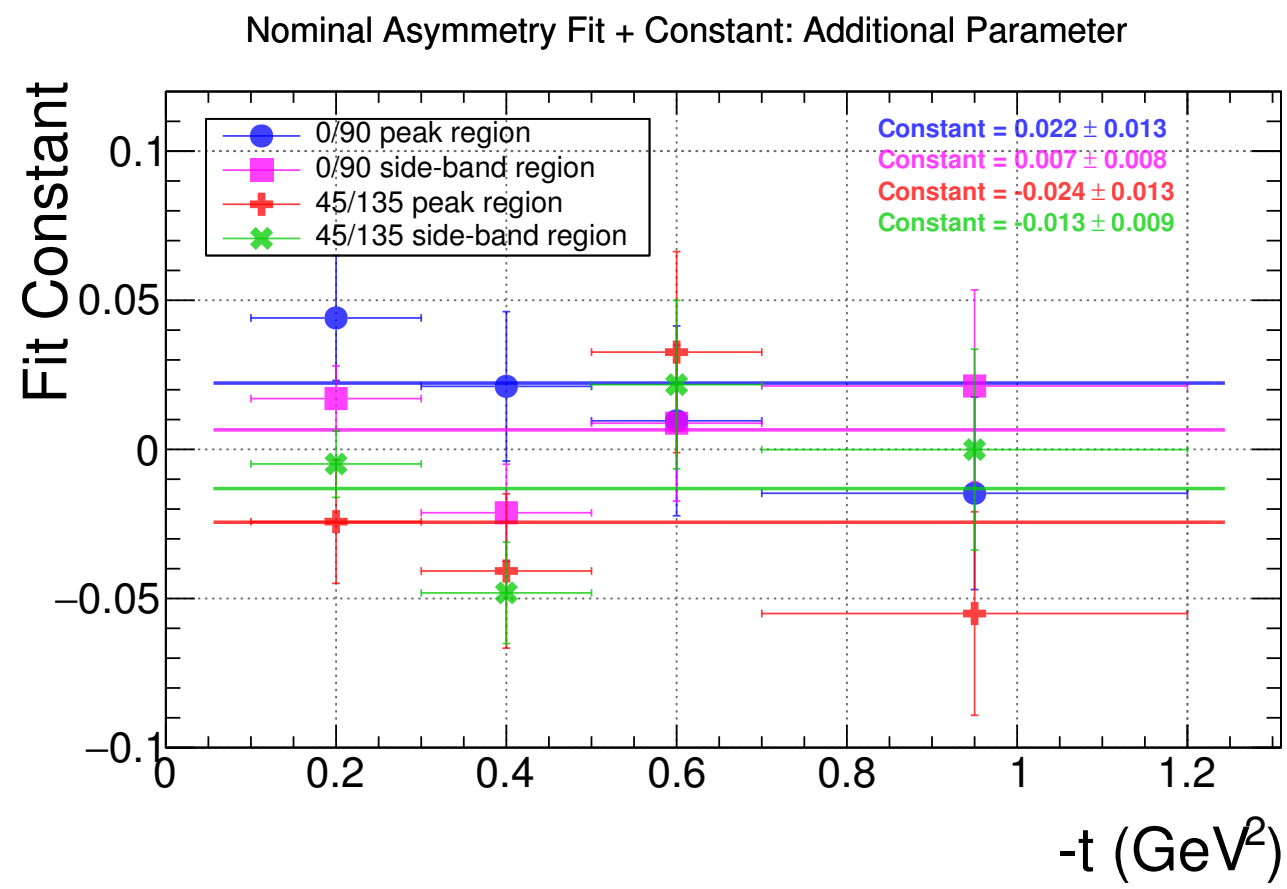

Figure 5.19: The fit parameter $A$ of the altered fit function in each $t$ bin for the $0 / 90$ peak region (blue circles) and side-band region (magenta squares), and for the 45/135 peak region (red crosses) and side-band region (green diagonal crosses). Fits to a constant function over each set of points show no systematic deviation of $A$ from 0 (colour online). 


\subsubsection{Finite $\phi$-Dependent Yield Bin Size}

Statistics in each channel dictate how many bins in $\phi$ the yields can be separated into in order to fit the yield asymmetry. With infinite statistics ${ }^{2}$, an arbitrarily large number of bins can be used, and a fit should capture the true $\Sigma$ asymmetry of the distribution, but with finite statistics, a finite number of bins must be used. With few bins, it is expected that the fit asymmetry may be biased. The effects on the extracted $\Sigma$ of varying the number of $\phi$ bins used for the yield asymmetry histograms was studied with Monte Carlo simulations [113]. $500 \mathrm{MC}$ samples were generated with a known beam polarization and $\Sigma=1$, then an asymmetry analysis was carried out with a varying number of $\phi$ bins for the yield distributions on each of them. For each number of $\phi$ bins studied, a distribution containing each of the 500 extracted $\Sigma$ fit parameters was made. Figure 5.20 shows the means (points) and widths (vertical error bars) of those distributions versus the number of $\phi$ bins used.

The effects of using 50 bins is on the order of a few tenths of a percent, seen as a deviation from unity in Figure 5.20, and the conservative estimate for a systematic uncertainty due to the finite number of bins quoted in Reference [113] was 0.5\%. For the $\eta^{\prime}$ analysis presented in this thesis, only 30 bins are being used. The MC study conducted did not probe a lower number of bins than 50, but based on the results presented in that study, a conservative systematic uncertainty of $1 \%$ is chosen for this $\eta^{\prime}$ asymmetry analysis.

\subsection{8 $\eta^{\prime}$ Systematics Summary}

The systematic error contributions discussed in this chapter to the average $\eta^{\prime} \Sigma$ beam asymmetry are summarized in Table 5.5 . These contributions to the systematic

\footnotetext{
${ }^{2}$ 'Infinite statistics' in a physics context means an amount of statistics such that the statistical error associated with an observable (the yield in each bin of $\phi$, in this case) is negligible.
} 


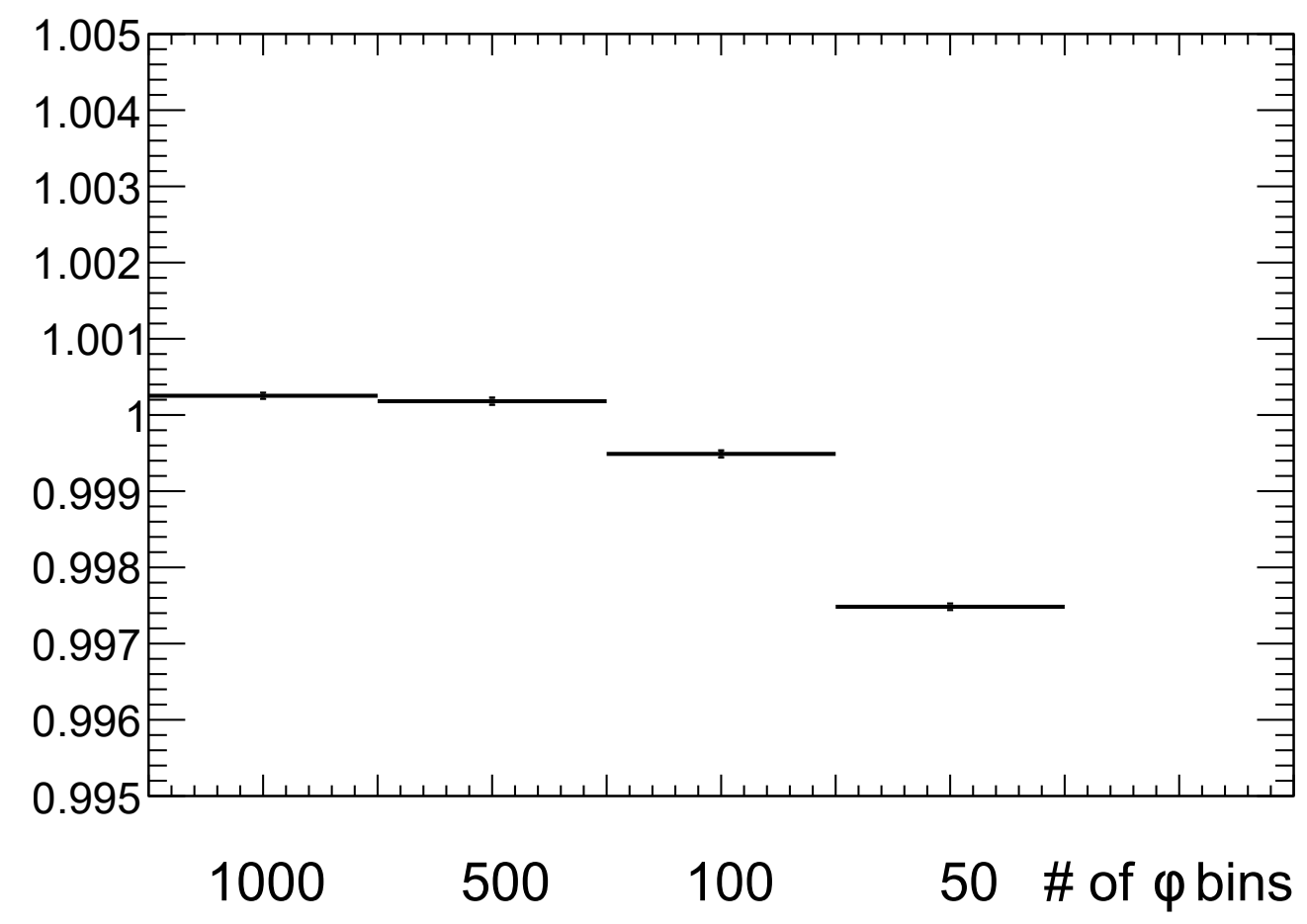

Figure 5.20: Means (points) and widths (vertical error bars) of the $\Sigma$ fit parameter distributions from fitting $500 \mathrm{MC}$ samples versus the number of bins used for the yield asymmetry histograms. For some points, the small vertical error bars are contained within the points. Figure from Reference [113].

uncertainty for the beam asymmetry of the $\eta^{\prime}$ are assumed to be uncorrelated ${ }^{3}$, so, for each $t$ bin, the systematic uncertainties are added in quadrature (given in Table 4.5) and are represented by the purple boxes on each point in Figure 4.30.

\begin{tabular}{|c||c|}
\hline Study & $\eta^{\prime}$ Systematic Error \\
\hline \hline$\Sigma_{\text {SB }}$ Determination & $\delta_{\text {SBSYS }}$ (Table 5.1) \\
\hline Event Selection Variations & $\delta_{\text {CUTSY }}$ (Table 5.3) \\
\hline Phase Offset Dependence & 0.006 \\
\hline Flux Ratio Dependence & 0.004 \\
\hline Finite $\phi$ Bin Size & 0.01 \\
\hline
\end{tabular}

Table 5.5: Summary of systematic uncertainties assigned to the average $\eta^{\prime} \Sigma$ beam asymmetry.

\footnotetext{
${ }^{3}$ No studies were conducted to validate this assumption. Because the statistical error in the analysis dominates over any systematic errors, the possible effects of correlated errors should be relatively small.
} 


\section{Chapter 6}

\section{Conclusions and Future Direction}

With data from the GlueX Experiment, the first measurements of the $\eta^{\prime}$ beam asymmetry at photon beam energies above $6 \mathrm{GeV}$ have been made, including evaluation of systematic uncertainties to do with detector measurements and analysis methods. These results are summarized in Appendix C. The measured asymmetry is statistically consistent with theoretical predictions from the JPAC theory group, and with more statistics from currently uncalibrated and future data, this model can be tested further. These statistics will also allow measurements of the beam asymmetry in the other major channels of the $\eta^{\prime}$ to check for consistency in results between analyses and topologies. The asymmetry of the $\eta$ has also been measured in the three major decay channels, giving results consistent with one another and consistent with past measurements by GlueX. However, these results show hints of deviation from theoretical models of the $\Sigma$ beam asymmetry for the $\eta$ and may help guide modifications to these models in future.

The next steps for the $\eta$ meson program at GlueX is to shift attentions towards Monte Carlo studies. Extraction of cross sections at high energy are the next major milestones for the $\eta$ and $\eta^{\prime}$ mesons, which becomes possible when accurate MC simulation of the detector is achieved. Studies into the acceptance of the GlueX detector 
for various decay topologies and careful matching of simulation to measured detector response bring constant improvements to cross section studies, and work within the collaboration is already underway towards extraction of the $\eta^{\prime}$ cross section in multiple decay channels [114].

Finally, once accurate MC simulations are available that faithfully recreate measured distributions and event selection techniques are developed to cleanly identify the simplest decay products of potential exotic and hybrid mesons ${ }^{1}$, partial wave analysis can be done. With the large body of statistics collected from the high-luminosity photon beam at GlueX, and because of the relatively high rate of production for hybrid mesons expected from a photoproduction experiment, comprehensive searches for evidence of the hybrid mesons claimed by other collaborations (and searches for other expected exotics and hybrids) using PWA should be possible. Mapping the entire light meson spectrum, including full multiplets of exotic and hybrid mesons, is the ultimate goal of GlueX, and simple studies, like measuring polarization observables or cross sections of known particles, are the necessary first steps towards this goal. In addition, measurements of cross sections and polarization observables provide valuable input to theorists for modelling production and exchange processes, and these theoretical models can help guide searches for exotic physics.

\footnotetext{
${ }^{1}$ In addition, problems that may be related to the shortcomings of the attempted asymmetry analyses using alternate decay channels of the $\eta^{\prime}$ (possible reconstruction issues with multi-photon final states, for instance) must be understood and corrected.
} 


\section{Bibliography}

[1] M. Gell-Mann, 'The Eightfold Way: A Theory of Strong Interaction Symmetry.' California Institute of Technology, USA (1961)

[2] M. Gell-Mann, 'The Symmetry Group of Vector and Axial Vector Currents.' Physics 1, 63 (1964)

[3] M. Gell-Mann and Y. Ne'eman, The Eightfold Way. W. A. Benjamin (1964)

[4] G. Zweig, 'An $\mathrm{SU}_{3}$ Model for Strong Interaction Symmetry and its Breaking.' CERN, report number CERN-TH-412 (1964)

[5] C. Meyer, 'Light and Exotic Mesons.' Lecture notes, Carnegie Mellon University, USA, web (2008)

[6] M. Tanabashi et al. (Particle Data Group), Physical Review D 98, 030001 (2018)

[7] A. Yamamoto, 'Fourier Analysis of the Flux-Tube Distribution in SU(3) Lattice QCD.' Physics Letters B 688, 345 (2010)

[8] G.S. Bali, K. Schilling, and C. Schlichter, 'OBSERVING LONG COLOR FLUX TUBES IN SU(2) LATTICE GAUGE THEORY.' Physical Review D 51, 5165 (1995)

[9] B. L. Ioffe, 'QCD at Low Energies.' Progress in Particle and Nuclear Physics 56, $232(2006)$

[10] F. Iddar and L. Semlala, 'Hybrid Mesons Masses in a Quark-Gluon Constituent Model.' hep-ph/0211289 (2002)

[11] W. Hou, C. Luo, and G. Wong, 'Glueball States in a Constituent Gluon Model.' Physical Review D 64, 014028 (2001)

[12] C.A. Meyer and E. S. Swanson, 'Hybrid Mesons.' Progress in Particle and Nuclear Physics 82, 21 (2015)

[13] J. J. Dudek and R. G. Edwards, 'Hybrid Baryons in QCD.' Physical Review D 85, $054016(2012)$ 
[14] J. J. Dudek, R. G. Edwards, P. Guo, and C. E. Thomas, 'Toward the Excited Isoscalar Meson Spectrum from Lattice QCD.' Physical Review D 88, 094505 (2013)

[15] L. Liu et al. (Hadron Spectrum Collaboration), 'Excited and Exotic Charmonium Spectroscopy from Lattice QCD.' Journal of High Energy Physics 2012, $126(2012)$

[16] R. G. Edwards, J. J. Dudek, D. G. Richards, and S. J. Wallace, 'Excited State Baryon Spectroscopy from Lattice QCD.' Physical Review D 84, 074508 (2011)

[17] E. Gregory et al., 'Towards the Glueball Spectrum from Unquenched Lattice QCD.' Journal of High Energy Physics 2012, JHEP10 (2012)

[18] F. Csikor, Z. Fodor, S. D. Katz, and T. G. Kovács, 'Pentaquark Hadrons from Lattice QCD.' Journal of High Energy Physics 2003, JHEP11 (2003)

[19] P. Junnarkar, N. Mathur, and M. Padmanath, 'Study of Doubly Heavy Tetraquarks in Lattice QCD.' arXiv:1810.12285 (2018)

[20] Y. Nambu, 'Quark Model and the Factorization of the Veneziano Amplitude.' Broken Symmetry: Selected Papers of Y. Nambu. Singapore: World Scientific Publishing (1995)

[21] Y. Nambu, 'QCD and the String Model.' Physics Letters B 80, 372 (1979)

[22] M Lüscher, K. Symanzik, and P. Weisz, 'Anomalies of the Free Loop Wave Equation in the WKB Approximation.' Nuclear Physics B 173, 365 (1980)

[23] A. M. Polyakov, 'Gauge Fields as Rings of Glue.' Nuclear Physics B 164, 171 (1980)

[24] N. Isgur, R. Kokoski, and J. E. Paton, 'Gluonic Excitations of Mesons: Why They Are Missing and Where to Find Them.' Physical Review Letters 54, 869 (1985)

[25] T. Barnes, F. E. Close, and E. S. Swanson, 'Hybrid and Conventional Mesons in the Flux Tube Model: Numerical Studies and their Phenomenological Implications.' Physical Review D 52, 5424 (1995)

[26] N. Isgur and J. Paton, 'Flux-Tube Model for Hadrons in QCD.' Physical Review D 31, 2910 (1985)

[27] T. J. Burns and F. E. Close, 'Hybrid-Meson Properties in Lattice QCD and Flux-Tube Models.' Physical Review D 74, 034003 (2006)

[28] X. Shen et al., 'Hybrid Mesons, Multiquarks and Molecular States.' $\tau$-Charm Physics at BES-III, Chapter 15, http://bes.ihep.ac.cn/bes3/phy_book/book/ book.html (2006) 
[29] J. J. Dudek, 'The lightest hybrid meson supermultiplet in QCD.' Physical Review D 84, 074023 (2011)

[30] P. R. Page, E. S. Swanson, and A. P. Szczepaniak, 'Hybrid meson decay phenomenology.' Physical Review D 59, 034016 (1999).

[31] D. Ebert, R. N. Faustov, and V. O. Galkin, 'Masses of Light Tetraquarks and Scalar Mesons in the Relativistic Quark Model.' The European Physical Journal C 60, 273 (2009)

[32] G. Eichmann, C. S. Fischer, and W. Heupel, 'The Light Scalar Mesons as Tetraquarks.' Physics Letters B 753, 282 (2016)

[33] I. J. R. Aitchison, 'Unitarity, Analyticity and Crossing Symmetry in Two- and Three-hadron Final State Interactions.' Lecture notes, SLAC National Accelerator Laboratory, USA, arXiv:1507.02697 (2015)

[34] K. Peters, 'A Primer on Partial Wave Analysis.' International Journal of Modern Physics A 21, 5618 (2006)

[35] C. Adolph et al. (COMPASS Collaboration), 'Resonance Production and $\pi \pi$ $S$-wave in $\pi^{-}+p \rightarrow \pi^{-} \pi^{-} \pi^{+}+p_{\text {recoil }}$ at $190 \mathrm{GeV} / c$.' Physical Review D 95, 032004 (2017)

[36] Yu. P. Gouz et al. (The VES Collaboration), 'Study of the Wave with $J^{P C}=$ $1^{-+}$in the Partial-Wave Analysis of $\eta^{\prime} \pi^{-}, \eta \pi^{-}, f_{1} \pi^{-}$, and $\rho^{0} \pi^{-}$Systems Produced in $\pi^{-} N$ Interactions at $p_{\pi^{-}}=37 \mathrm{GeV} / c$.' AIP Conference Proceedings 272, 572 (1992)

[37] Yu. Khokhlov et al. (The VES Collaboration), 'Study of $X(1600) 1^{-+}$Hybrid.' Nuclear Physics A 663-664, 596 (2000)

[38] A. Zaitsev et al. (The VES Collaboration), 'Study of Exotic Resonances in Diffractive Reactions.' Nuclear Physics A 675, 155 (2000)

[39] G. S. Adams et al. (The E852 Collaboration), 'Observation of a New $J^{P C}=1^{-+}$ Exotic State in the Reaction $\pi^{-} p \rightarrow \pi^{+} \pi-\pi-p$ at $18 \mathrm{GeV} / c$.' Physical Review Letters 81, 5760 (1998)

[40] E. I. Ivanov et al. (The E852 Collaboration), 'Observation of Exotic Meson Production in the Reaction $\pi^{-} p \rightarrow \eta^{\prime} \pi^{-} p$ at $18 \mathrm{GeV} / c$.' Physical Review Letters 86, 3977 (2001)

[41] S. U. Chung et al. (The E852 Collaboration), 'Exotic and $q \bar{q}$ Resonances in the $\pi^{+} \pi^{-} \pi^{-}$System Produced in $\pi^{-} p$ Collisions at $18 \mathrm{GeV} / c$.' Physical Review D 65, 072001 (2002)

[42] J. Khun et al. (The E852 Collaboration), 'Exotic Meson Production in the $\mathrm{f}_{1}(1285) \pi^{-}$System Observed in the Reaction $\pi^{-} p \rightarrow \eta \pi^{+} \pi-\pi-p$ at 18 GeV/c.' Physics Letters B 595, 109 (2004) 
[43] M. Lu et al. (The E852 Collaboration), 'Exotic Meson Decay to $\omega \pi^{0} \pi^{-}$.' Physical Review Letters 94, 032002 (2005)

[44] M. G. Alekseev et al. (The COMPASS Collaboration), 'Observation of a $J^{P C}=1^{-+}$Exotic Resonance in Diffractive Dissociation of $190 \mathrm{GeV} / c \pi^{-}$into $\pi^{-} \pi^{-} \pi^{+}$.' Physical Review Letters 104, 241803 (2010)

[45] B. Grube et al. (The COMPASS Collaboration), 'Precision Meson Spectroscopy at COMPASS.' EPJ Web of Conferences 3, 07018 (2010)

[46] F. Nerling et al. (The COMPASS Collaboration), 'Spin-exotic Search in the $\rho \pi$ Decay Channel: New Results on $\pi^{-} \pi^{0} \pi^{0}$ in Comparison to $\pi^{-} \pi^{+} \pi^{-}$Final States (Diffractively Produced on Proton).' In Proceedings of the XIV International Conference on Hadron Spectroscopy (Hadron2011), Munich, eConf C110613 (2011)

[47] G. S. Adams et al. (The CLEO Collaboration), 'Amplitude Analysis of the Decays $\chi_{c 1} \rightarrow \eta \pi^{+} \pi^{-}$and $\chi_{c 1} \rightarrow \eta^{\prime} \pi^{+} \pi^{-}$.' Physical Review D 84, 112009 (2011)

[48] C. A. Baker et al., 'Confirmation of $a_{0}(1450)$ and $\pi_{1}(1600)$ in $\bar{p} p \rightarrow \omega \pi^{+} \pi^{-} \pi^{0}$ at Rest.' Physics Letters B 563, 140 (2003)

[49] M. Nozar et al. (The CLAS Collaboration), 'Search for the Photoexcitation of Exotic Mesons in the $\pi^{+} \pi^{+} \pi^{-}$System.' Physical Review Letters 102, 102002 (2009)

[50] B. A. Mecking et al. (The CLAS Collaboration), 'The CEBAF Large Acceptance Spectrometer (CLAS).' Nuclear Instruments and Methods A 503, Issue 3, 513 (2003)

[51] A. R. Dzierba et al., 'Partial Wave Analysis of the $\pi^{-} \pi^{-} \pi^{+}$and $\pi^{-} \pi^{0} \pi^{0}$ Systems and the Search for a $J^{P C}=1^{-+}$Meson.' Physical Review D 73, 072001 (2006)

[52] A. R. Dzierba et al., 'Study of the $\eta \pi^{0}$ Spectrum and Search for a $J^{P C}=1^{-+}$ Exotic Meson.' Physical Review D 67, 094015 (2003)

[53] T. Schlúter (The COMPASS Collaboration), 'The Exotic eta'pi- Wave in 190 GeV pi-p -> pi-eta'p at COMPASS.' In Proceedings of the XIV International Conference on Hadron Spectroscopy (hadron2011), Munich, eConf C110613 (2011)

[54] D. V. Amelin et al. (VES Collaboration), 'Study of Resonance Production in Diffractive Reaction pi- A $\longrightarrow$ pi+ pi- pi- A.' Physics Letters B 356, 595 (1995)

[55] D. Barberis et al. (WA102 Collaboration), 'A Study of the Centrally Produced pi+ pi- pi+ pi- Channel in p p Interactions at 450-GeV/c.' Physics Letters $B$ 413, 217 (1997) 
[56] A. Donnachie and Yu. S. Kalashinikova, 'Hunting the Vector Hybrid.' Physical Review D 60, 114011 (1999)

[57] E. Smith, 'GlueX: Photoproduction of Hybrid Mesons.' EPJ Web of Conferences 37, 01026 (2012)

[58] D. S. Carman, 'GlueX: the Search for Gluonic Excitations at Jefferson Laboratory.' AIP Conference Proceedings 814, 173 (2006)

[59] V. Mathieu et al., 'On the $\eta$ and $\eta$ 'Photoproduction Beam Asymmetry at High Energies.' Physics Letters B 774, 362 (2017)

[60] E. Chudakov, 'Measurement of the $\mathrm{J} / \psi$ Photoproduction Cross Section Close to Threshold.' SPIN2018 Conference Proceedings (2018)

[61] S. J. Brodsky, E. Chudakov, P. Hoyer, and J. M. Laget, 'Photoproduction of Charm Near Threshold.' Physics Letters B 498, 23 (2001)

[62] H. Al Ghoul et al. (The GlueX Collaboration), 'First Results from the GlueX Experiment.' AIP Conference Proceedings 1735, 020001 (2016)

[63] H. Al Ghoul et al. (The GlueX Collaboration), 'Measurement of the Beam Asymmetry $\Sigma$ for $\pi^{0}$ and $\eta$ Photoproduction on the Proton at $E_{\gamma}=9 \mathrm{GeV}$.' Physical Review C 95, 042201 (2017)

[64] B. Dey, M. E. McCracken, D. G. Ireland, and C. A. Meyer, 'Polarization Observables in the Longitudinal Basis for Pseudo-scalar Meson Photoproduction Using a Density Matrix Approach.' Physical Review C 83, 055208 (2011)

[65] K. Schilling, P. Seyboth, and G. Wolf, 'On the Analysis of Vector-meson Production by Polarized Photons.' Nuclear Physics B 15, 397 (1970)

[66] I. S. Barker, A. Donnachie, and J. K. Storrow, 'Complete Experiments in Pseudoscalar Photoproduction.' Nuclear Physics B 95, 347 (1975)

[67] D. Ho et al. (The CLAS Collaboration), 'Beam-Target Helicity Asymmetry for $\vec{\gamma} \vec{n} \rightarrow \pi^{-} p$ in the $N^{*}$ Resonance Region.' Physical Review Letters 118, 242002 (2017)

[68] P. Collins et al. (The CLAS Collaboration), 'Photon Beam Asymmetry $\Sigma$ for $\eta$ and $\eta^{\prime}$ Photoproduction from the Proton.' Physics Letters B 771, 213 (2017)

[69] P. Collins et al. (The CLAS Collaboration), 'Photon Beam Asymmetry $\Sigma$ in the Reaction $\vec{\gamma} p \rightarrow p \omega$ for $E_{\gamma}=1.152$ to $1.876 \mathrm{GeV}$.' Physics Letters $B \mathbf{7 7 3}$, $112(2017)$

[70] P. Roy et al. (The CLAS Collaboration), 'Measurement of the Beam Asymmetry $\Sigma$ and the Target Asymmetry $T$ in the Photoproduction of $\omega$ Mesons off the Proton Using CLAS at Jefferson Laboratory.' Physical Review C 97, 055202 (2018) 
[71] J. Bono et al. (The CLAS Collaboration), 'First Measurement of $\Xi^{-}$Polarization in Photoproduction.' Physics Letters B 783, 280 (2018)

[72] D. H. Ho et al. (The CLAS Collaboration), 'Beam-Target Helicity Asymmetry $E$ in $K^{0} \Lambda$ and $K^{0} \Sigma^{0}$ Photoproduction on the Neutron.' Physical Review $C$ 98, 045205 (2018)

[73] P. D. B. Collins, An Introduction to Regge Theory and High Energy Physics. Cambridge: Cambridge University Press (1977)

[74] R. L. Anderson, D. B. Gustavson, J. R. Johnson, I. D. Overman, D. M. Ritson, B. H. Wiik, and D. Worcester, 'High-Energy $\pi^{0}$ Photoproduction from Hydrogen with Unpolarized and Linearly Polarized Photons.' Physical Review D 4, 1937 (1971)

[75] J. M. Laget, 'The Primakoff Effect on a Proton Target.' Physical Review C 72, $022202(2005)$

[76] J. M. Laget, 'Unitarity Constraints on Neutral Pion Electroproduction.' Physics Letters B 695, 199 (2011)

[77] V. Mathieu, G. Fox, and A. P. Szczepaniak, 'Nuetral Pion Photoproduction in a Regge Model.' Physical Review D 92, 074013 (2015)

[78] J. Nys, V. Mathieu, C. Fernández-Ramírez, A. N. Hiller Blin, A. Jackura, M. Mikhasenko, A. Pilloni, A. P. Szczepaniak, G. Fox, and J. Ryckebusch (Joint Physics Analysis Center), 'Finite-energy Sum Rules in Eta Photoproduction off a Nucleon.' Physical Review D 95, 034014 (2017)

[79] A. Donnachie and Yu. S. Kalashnikova, 'Photoproduction of $a_{0}(980)$ and $f_{0}(980)$.' Physical Review C 93, 025203 (2016)

[80] G. R. Goldstein and J. F. Owens, III, ' $\pi^{0}$ Photoproduction in a Weak-Regge-Cut Model.' Physical Review D 7, 865 (1973)

[81] C. Reece, 'Continuous Wave Superconducting Radio Frequency Electron Linac for Nuclear Physics Research.' Physical Review Accelerators and Beams. 19, 124801 (2016).

[82] G. Ciovati, 'TM-Class Cavity Design.' Talk presented at USPAS 2015, East Brunswick, NJ, USA (2015)

[83] F. Gerigk, 'Cavity Types.' Proceedings, CAS - CERN Accelerator School: RF for Accelerators. CERN, report number CERN-2011-007, 277 (2011)

[84] R. Kazimi et al., 'Four Beam Generation for Simultaneous Four-Hall Operation at CEBAF.' Paper presented at IPAC2016, Busan, Korea. JLab, Newport News, VA, USA (2016). 
[85] A. W. Sàenz and H. Überall, Coherent Radiation Sources. Berlin Heidelberg: Springer-Verlag (1985)

[86] E. Haug and W. Nakel, The Elementary Process of Bremsstrahlung. Singapore: World Scientific Publishing Co. Pte. Ltd. (2004)

[87] R. T. Jones, 'Intense Beams of Polarized and Nearly Monochromatic Photons from Coherent Bremsstrahlung.' University of Connecticut, USA, web (1997)

[88] GlueX Wiki contributors, 'Diamond Radiators.' On the public GlueX Wiki at https://halldweb.jlab.org/wiki/index.php/Diamond_radiators (last modified March, 2016)

[89] The GlueX Collaboration, 'Hall D / GlueX Technical Design Report.' Jefferson Lab, VA (2014)

[90] R. T. Jones, 'GlueX Photon Source Characteristics as a Function of Electron Beam Energy.' From the GlueX Document Database, Document \#384 (2004)

[91] M. Dugger et al., 'Design and Construction of a High-Energy Photon Polarimeter.' Nuclear Instruments and Methods A 876, 115 (2017)

[92] F. Barbosa, C. Hutton, A. Sitnikov, A. Somov, S. Somov, and I. Tolstukhin, 'Pair Spectrometer Hodoscope for Hall D at Jefferson Lab.' Nuclear Instruments and Methods A 795, 376 (2015)

[93] C. Keith, 'Targets for a Neutral Kaon Beam.' Paper presented at JLab Workshop: Physics with Neutral Kaon Beam, Newport News, VA, USA (2016)

[94] C. Meyer, 'Physics and Detector Performance Metrics for the GlueX Experiment.' From the GlueX Document Database, Document \#1063 (2008)

[95] Y. van Haarlem et al., 'The GlueX Central Drift Chamber: Design and Performance.' Nuclear Instruments and Methods A 622, 142 (2010)

[96] C. Meyer and Y. van Haarlem, 'The GlueX/HallD Central Drift Chamber.' Carnegie Mellon University, PA (2008)

[97] D. S. Carman and S. Taylor, 'Hall D Forward Drift Chamber Technical Design Report.' Jefferson Lab, VA (2008)

[98] T. D. Beattie et al., 'Construction and Performance of the Barrel Electromagnetic Calorimeter for the GlueX Experiment.' Nuclear Instruments and Methods A 896, 24 (2018)

[99] M. Ramilli, 'Characterization of SiPM: Temperature dependencies.' IEEE Nuclear Science Symposium Conference Record 2008, 2467 (2008)

[100] E. G. Anassontzis et al., 'Relative Gain Monitoring of the GlueX Calorimeters.' Nuclear Instruments and Methods A 738, 41 (2014) 
[101] K. Moriya et al., 'A Measurement of the Energy and Timing Resolution of the GlueX Forward Calorimeter Using and Electron Beam.' Nuclear Instruments and Methods A 726, 60 (2013)

[102] M. Patsyuk, 'GlueX Overview: Status and Some Future Plans.' EPJ Web of Conferences 138, 01029 (2017)

[103] E. Pooser et al., 'The GlueX Start Counter Detector.' Nuclear Instruments and Methods A 927, 330 (2019)

[104] GlueX Wiki contributors, 'Time-of-Flight.' On the public GlueX Wiki at https://halldweb.jlab.org/wiki/index.php/Time-of-Flight (last modified June, 2018)

[105] E. Smith, 'Time-of-Flight Measurements with the Barrel Calorimeter and the Forward Scintillators.' From the GlueX Document Database, Document \#1001 (2008)

[106] I. Adam et al. (BaBar DIRC Collaboration), 'The DIRC Particle Identification System for the BaBar Experiment.' Nuclear Instruments and Methods A 538, $281(2005)$

[107] J. Stevens et al., 'The GlueX DIRC Project.' Journal of Instrumentation 11, C07010 (2016)

[108] GlueX Wiki contributors, 'Spring 2017 Analysis Launch Cuts.' On the public GlueX Wiki at https://halldweb.jlab.org/wiki/index.php/Spring_2017_Analysis _Launch_Cuts (last modified August, 2018)

[109] D. Keller, 'Techniques in Kinematic Fitting.' CLAS-NOTE 2010-015, web (2010)

[110] N. Gagunashvili, ' $\sigma^{2}$ Test for Comparison of Weighted and Unweighted Histograms.' In Proceedings of PHYSTAT05, Oxford, UK (2006)

[111] M. Baalouch, 'Eta' -> EtaPi0Pi0 Selection.' From the GlueX Document Database, Document \#3334 (2017)

[112] A. Somov, A. Tolstukhin, S. V. Somov, and V. V. Berdnikov, 'Commissioning of the Pair Spectrometer of the GlueX Experiment.' Journal of Physics: Conference Series 798, 012175 (2017)

[113] D. Mack, J. R. Stevens, I. Strakovsky, S. Taylor, and Z. Zhang, 'Analysis Note: Measurement of the Beam Asymmetry $\Sigma$ for $\pi^{0}$ and $\eta$ Photoproduction on the Proton at $E_{\gamma}=9 \mathrm{GeV}$.' From the GlueX Document Database, Document \#3118 (2017)

[114] G. Vasileiadis, 'The Eta Pi Pi Analysis and the Eta' Cross Section.' From the GlueX Document Database, Document \#3861 (2019) 


\section{Appendix A}

\section{Nominal Event Selection Cuts}

- Number of Extra Charged Tracks $\leq 3$

- Standard CDC dE/dx Cuts (low-momentum $\pi /$ proton separation)

- Particle Timing Cuts (see Table 3.1)

- Missing Mass: $|\mathrm{MM}|^{2}<0.05 \mathrm{GeV}^{2}$

- Reconstructed Vertex: $52 \mathrm{~cm}<\mathrm{z}<78 \mathrm{~cm}$

- Reconstructed Vertex: $\mathrm{r}<1.0 \mathrm{~cm}$

- FCAL Fiducial Cut: $25 \mathrm{~cm}>\mathrm{r}_{\gamma}^{(\mathrm{FCAL})}>100 \mathrm{~cm}$

- BCAL Fiducial Cut: $\mathrm{z}_{\gamma}^{(\mathrm{BCAL})}<380 \mathrm{~cm}$

- Recoil Proton Momentum: $\mathrm{P}_{\text {recoil }}>0.25 \mathrm{GeV} / c$

- $0.60 \mathrm{GeV}<$ Decaying $\eta^{\prime}$ Mass $<1.30 \mathrm{GeV}$

- $0.46 \mathrm{GeV}<$ Decaying $\eta$ Mass $<0.62 \mathrm{GeV}$

- $0.105 \mathrm{GeV}<$ Decaying $\pi^{0}$ Mass $<0.165 \mathrm{GeV}$

- RF Matching, 'Peak' events: $\left|\Delta \mathrm{t}_{\mathrm{RF}}\right|<2.004 \mathrm{~ns}$

- RF Matching, 'Accidental' events: $6.012 \mathrm{~ns}<\left|\Delta \mathrm{t}_{\mathrm{RF}}\right|<18.036 \mathrm{~ns}$

- Kinematic Fit CL Cut: CL > $10^{-5}$

- Beam Energy for Asymmetry Analyses: $8.2 \mathrm{GeV}<E_{\gamma}<8.8 \mathrm{GeV}$ 


\section{Appendix B}

\section{Additional Analysis Plots}
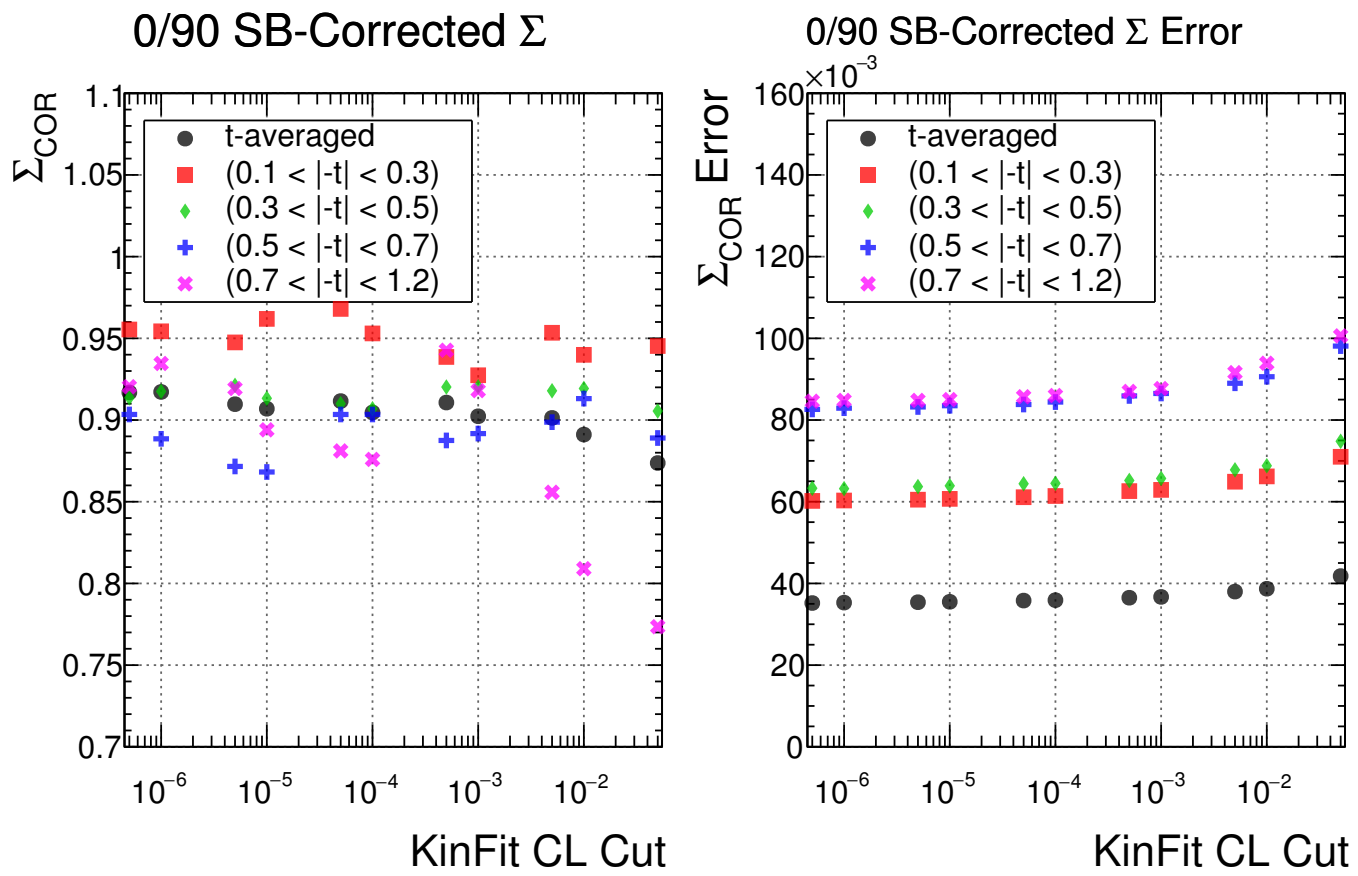

Figure B.1: $\eta \rightarrow \pi^{+} \pi^{-} \pi^{0}$ 0/90 SB-corrected $\Sigma$ asymmetries for the studied bins of $t$ versus kinematic fit confidence level cut (left) and statistical errors on those asymmetries (right) (colour online). 

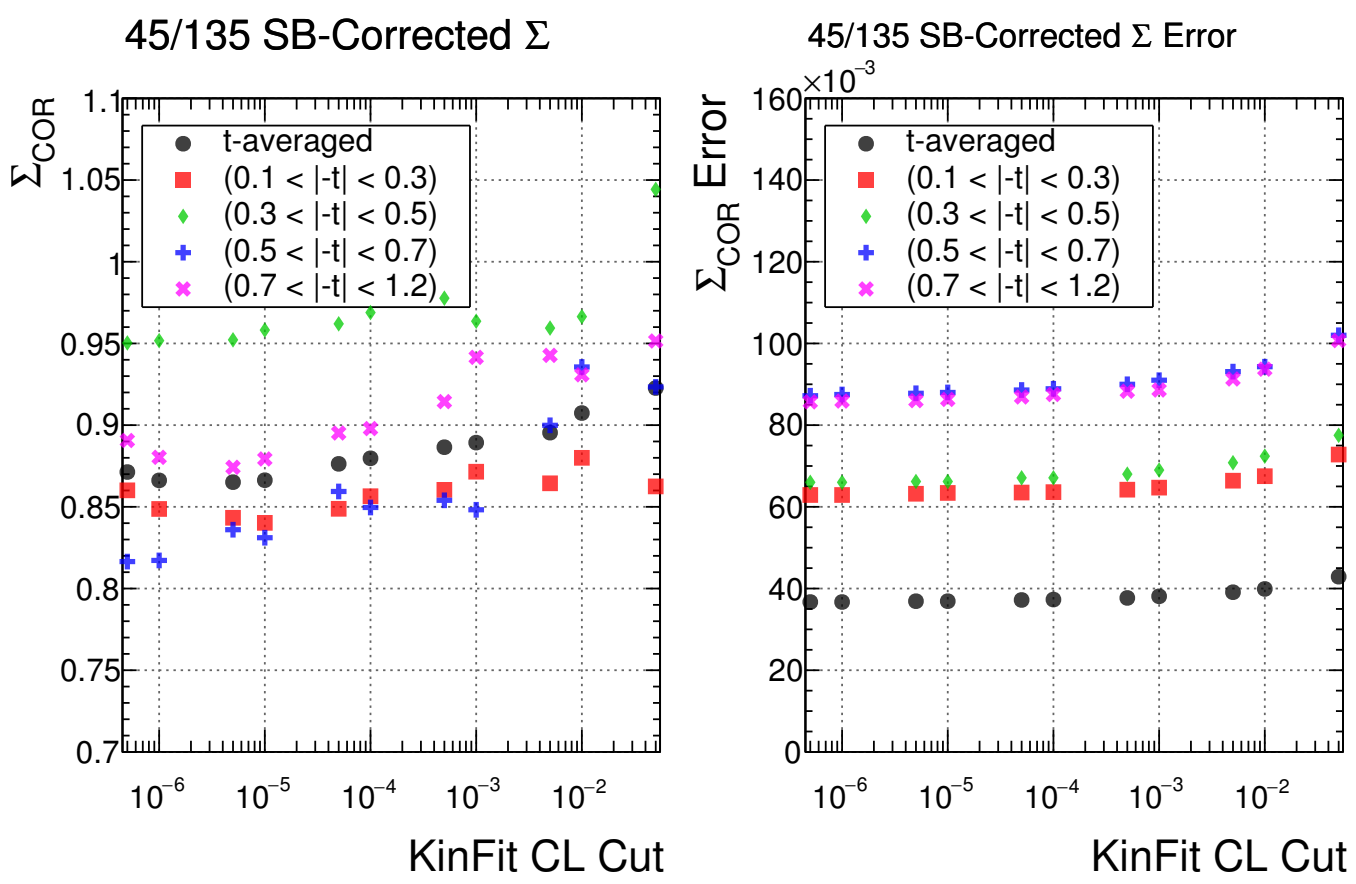

Figure B.2: $\eta \rightarrow \pi^{+} \pi^{-} \pi^{0}$ 45/135 SB-corrected $\Sigma$ asymmetries for the studied bins of $t$ versus kinematic fit confidence level cut (left) and statistical errors on those asymmetries (right) (colour online).
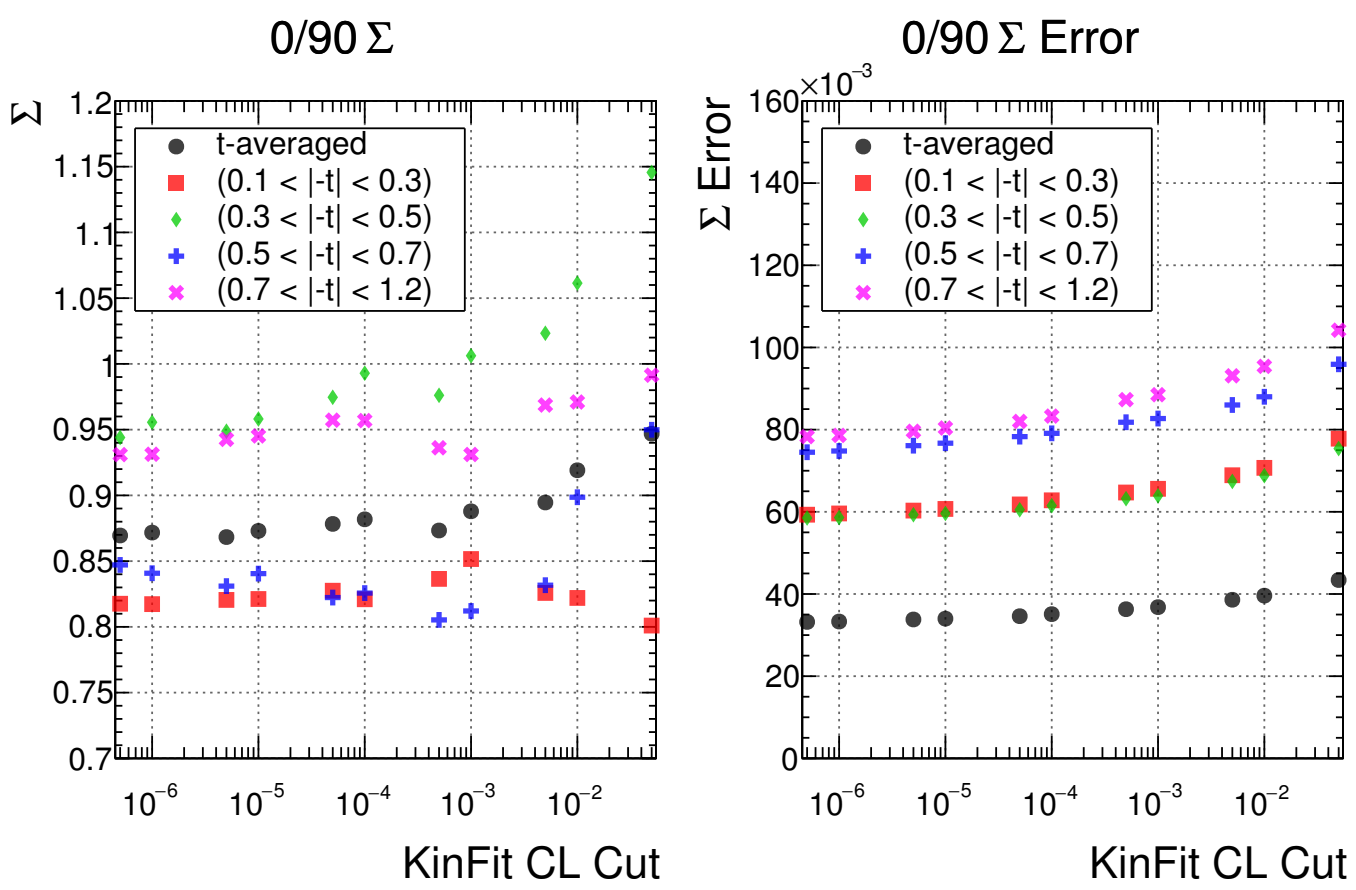

Figure B.3: $\eta \rightarrow 3 \pi^{0}$ 0/90 SB-corrected $\Sigma$ asymmetries for the studied bins of $t$ versus kinematic fit confidence level cut (left) and statistical errors on those asymmetries (right) (colour online). 

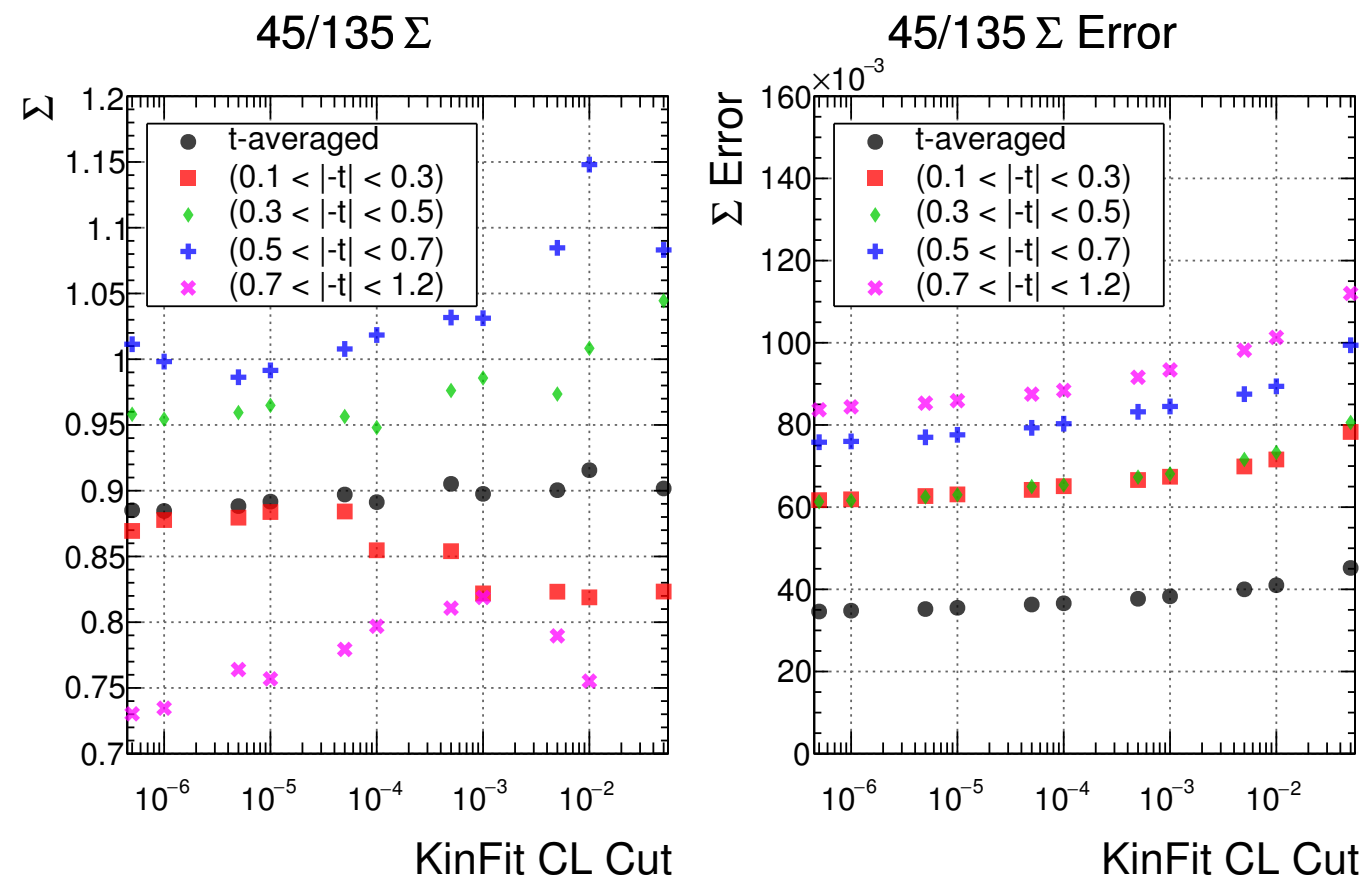

Figure B.4: $\eta \rightarrow 3 \pi^{0} 45 / 135$ SB-corrected $\Sigma$ asymmetries for the studied bins of $t$ versus kinematic fit confidence level cut (left) and statistical errors on those asymmetries (right) (colour online).
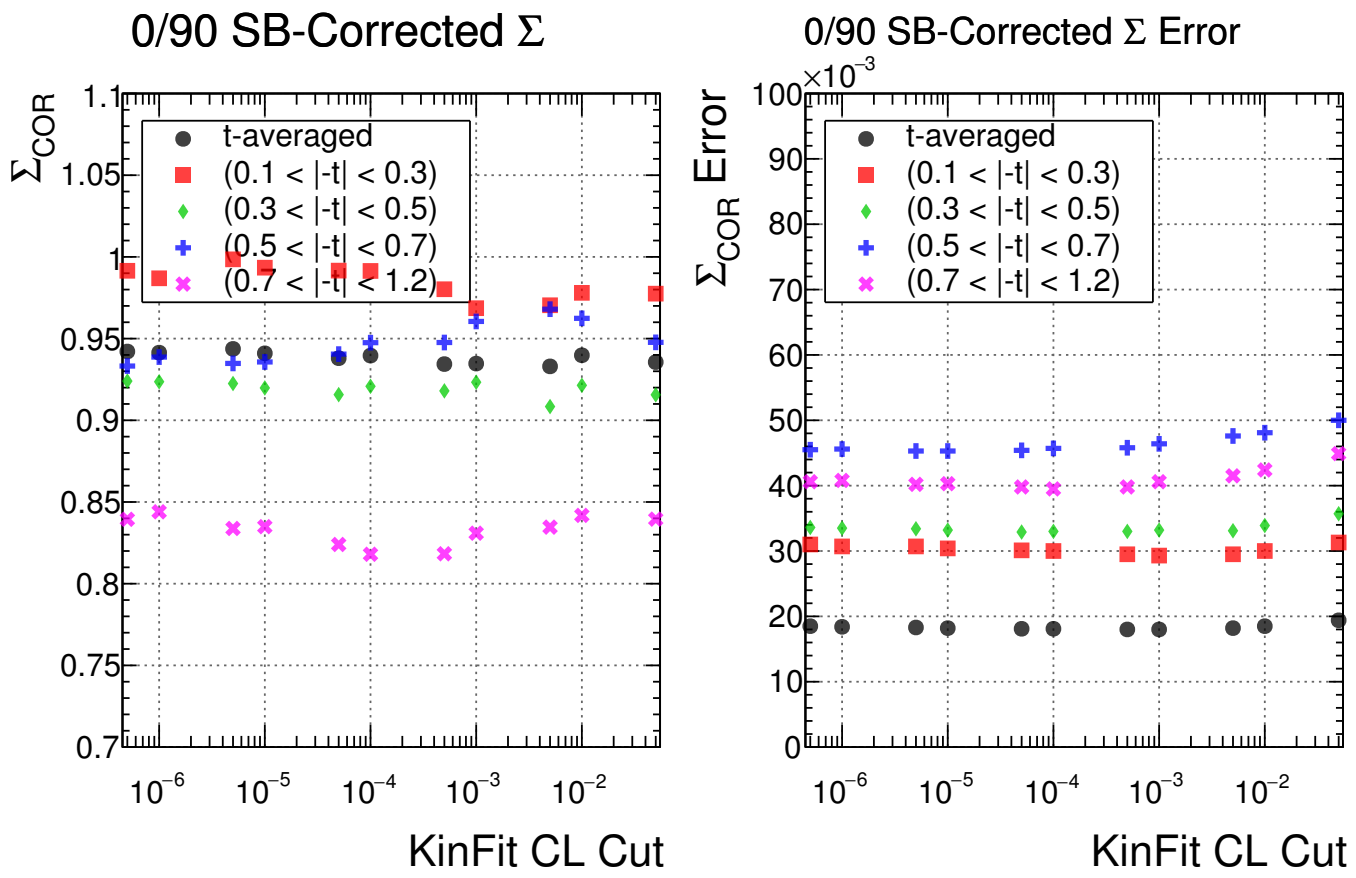

Figure B.5: $\eta \rightarrow 2 \gamma$ 0/90 SB-corrected $\Sigma$ asymmetries for the studied bins of $t$ versus kinematic fit confidence level cut (left) and statistical errors on those asymmetries (right) (colour online). 

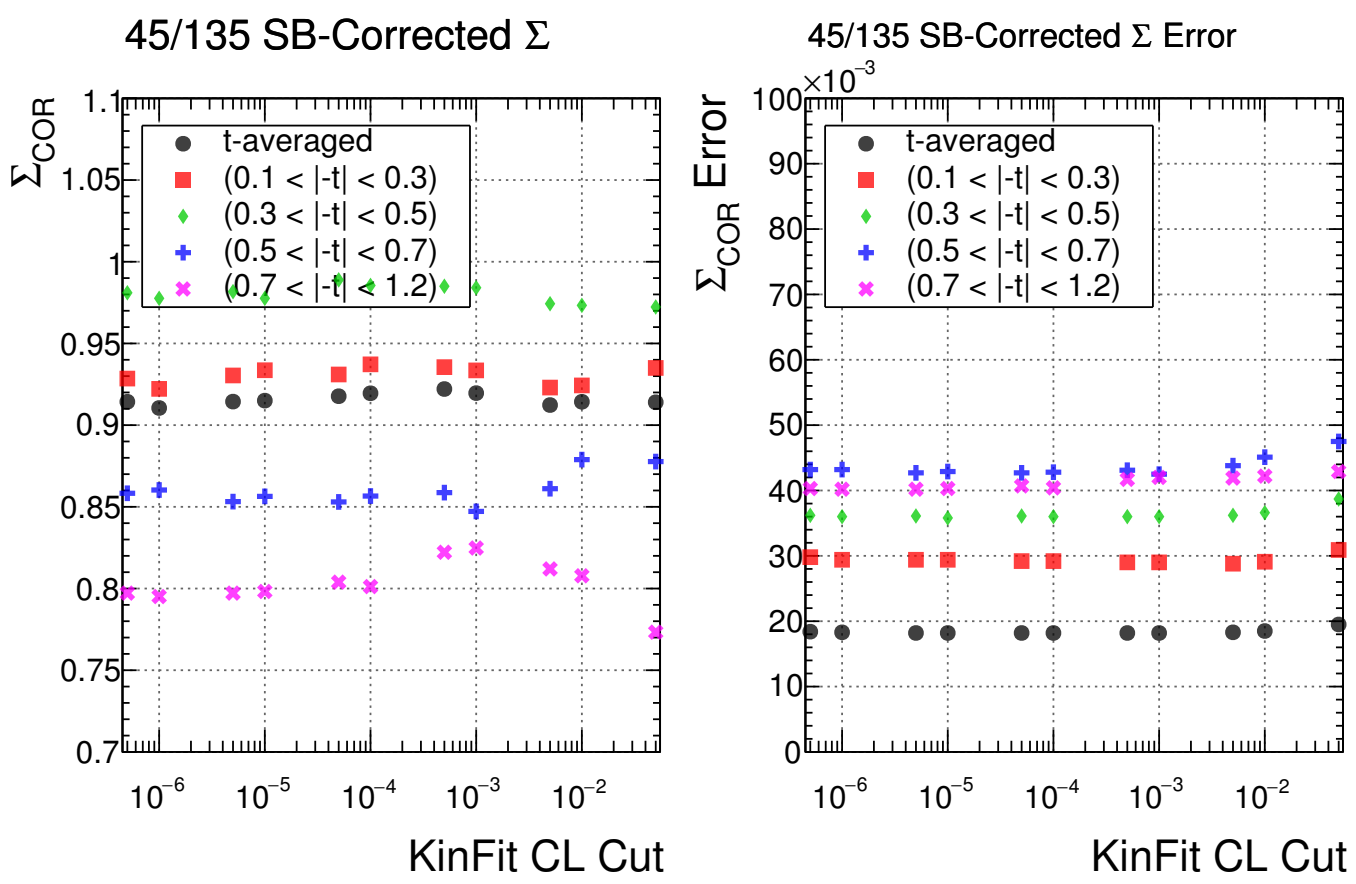

Figure B.6: $\eta \rightarrow 2 \gamma$ 45/135 SB-corrected $\Sigma$ asymmetries for the studied bins of $t$ versus kinematic fit confidence level cut (left) and statistical errors on those asymmetries (right) (colour online).
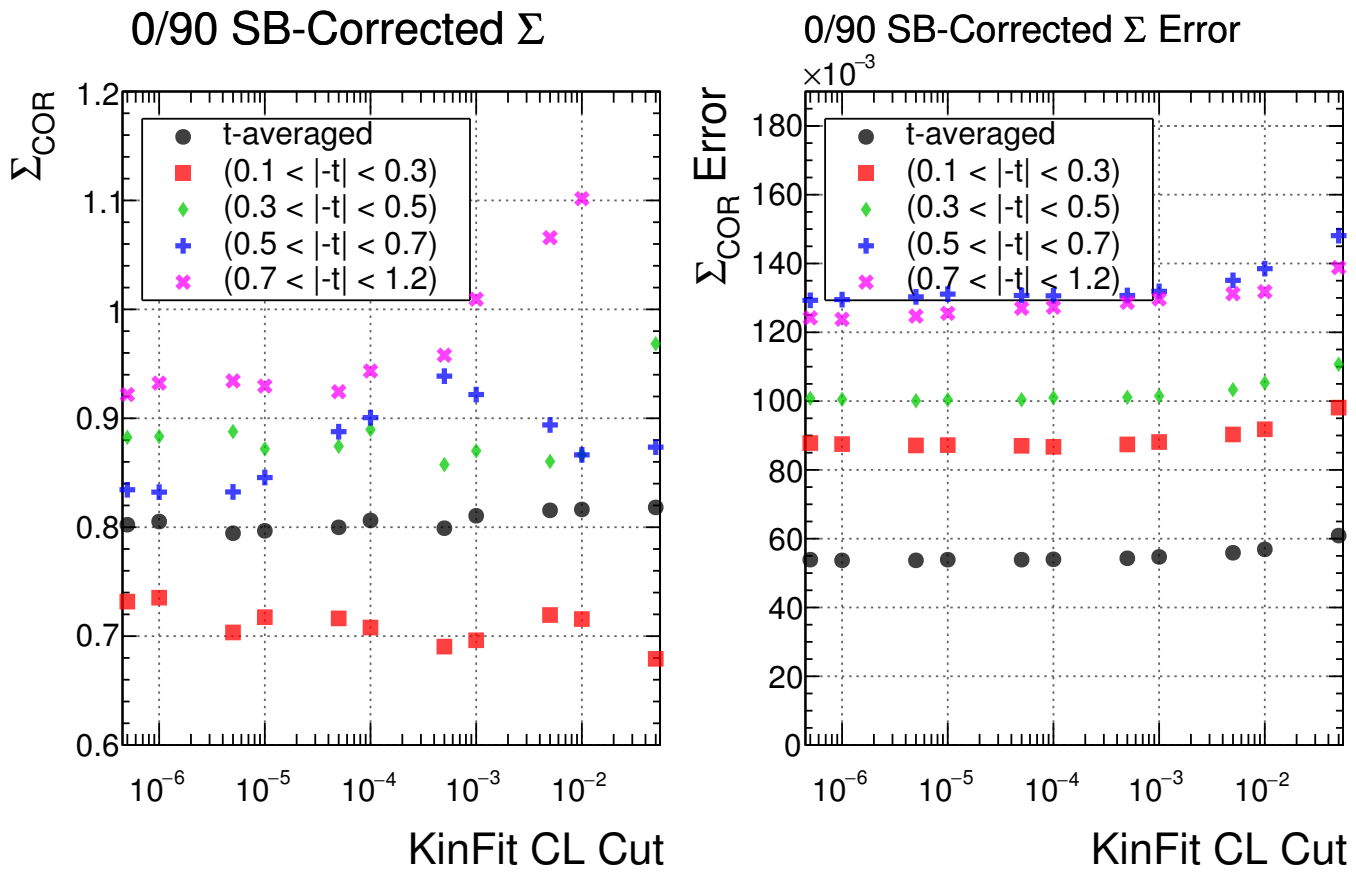

Figure B.7: $\eta^{\prime} \rightarrow \pi^{+} \pi^{-} \eta$ 0/90 SB-corrected $\Sigma$ asymmetries for the studied bins of $t$ versus kinematic fit confidence level cut (left) and statistical errors on those asymmetries (right) (colour online). 

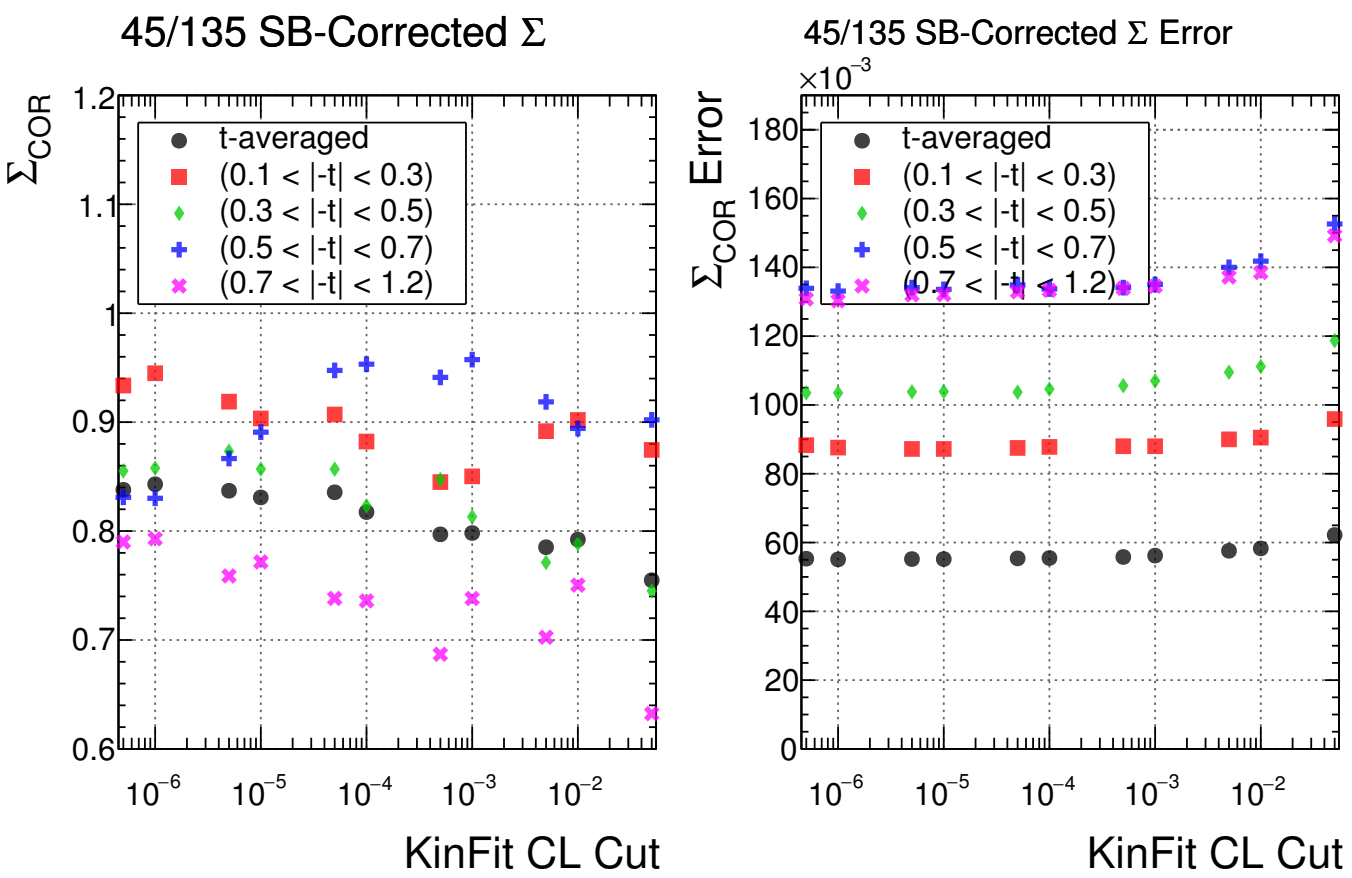

Figure B.8: $\eta^{\prime} \rightarrow \pi^{+} \pi^{-} \eta$ 45/135 SB-corrected $\Sigma$ asymmetries for the studied bins of $t$ versus kinematic fit confidence level cut (left) and statistical errors on those asymmetries (right) (colour online).

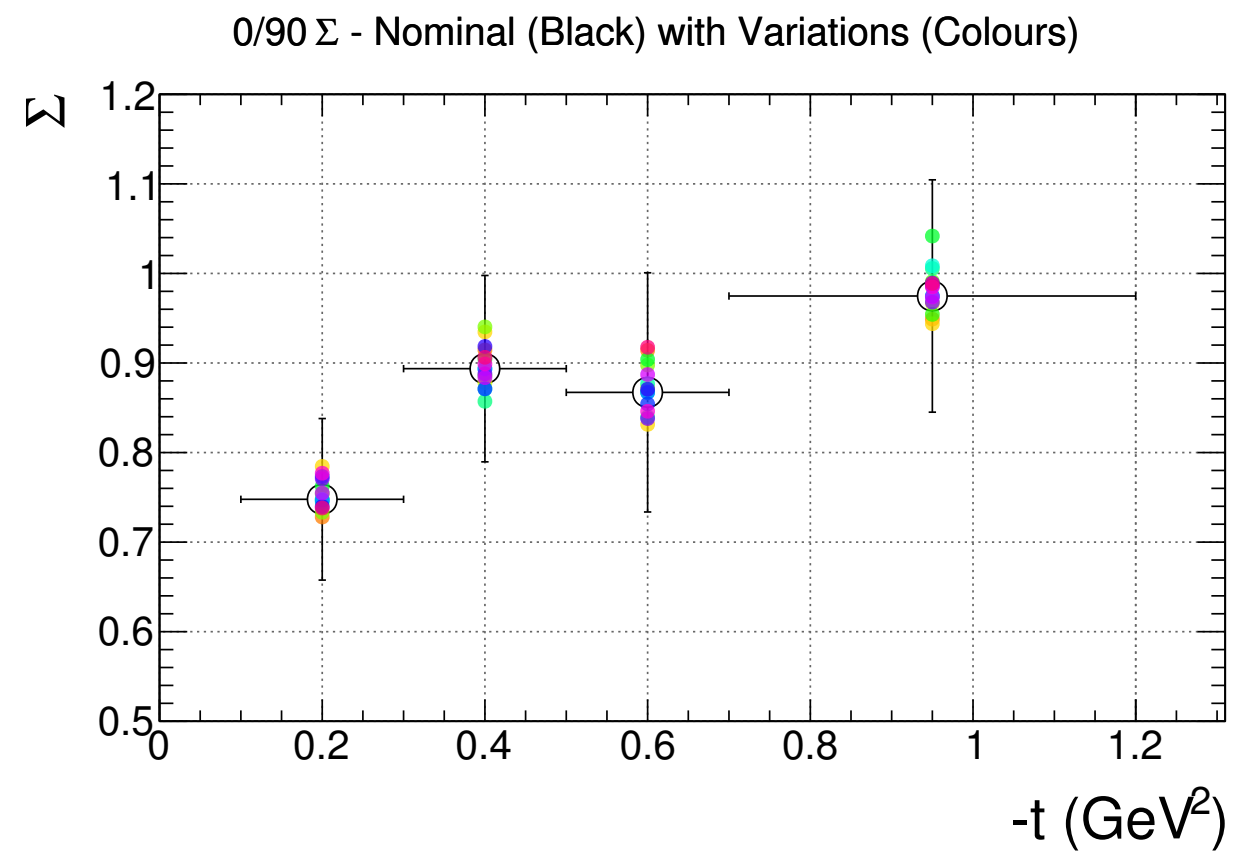

Figure B.9: 0/90 $\eta^{\prime}$ SB-corrected asymmetries for nominal (black open circles with error bars) and varied (coloured filled circles) cuts. Colours are as in the legend shown in Figure 5.12 (colour online). 


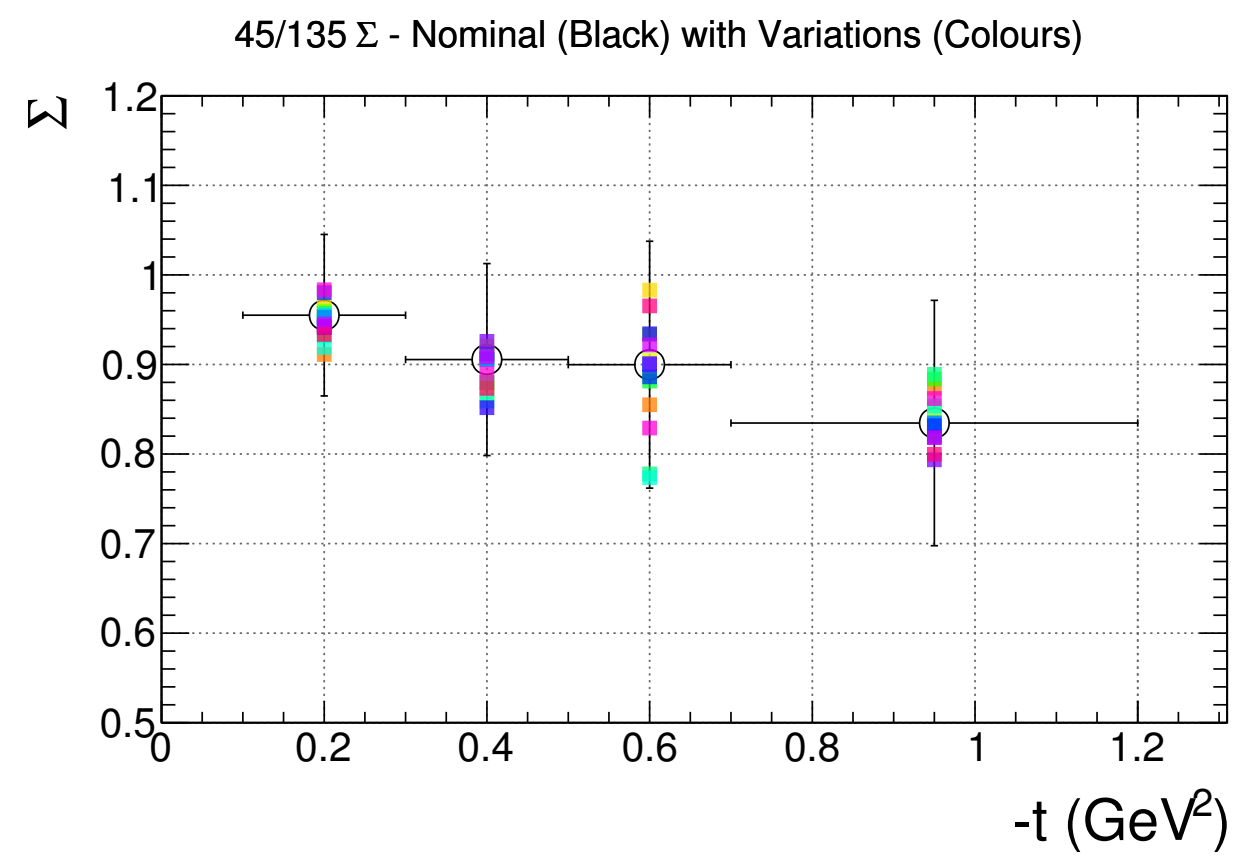

Figure B.10: 45/135 $\eta^{\prime}$ SB-corrected asymmetries for nominal (black open circles with error bars) and varied (coloured filled circles) cuts. Colours are as in the legend shown in Figure 5.12 (colour online).

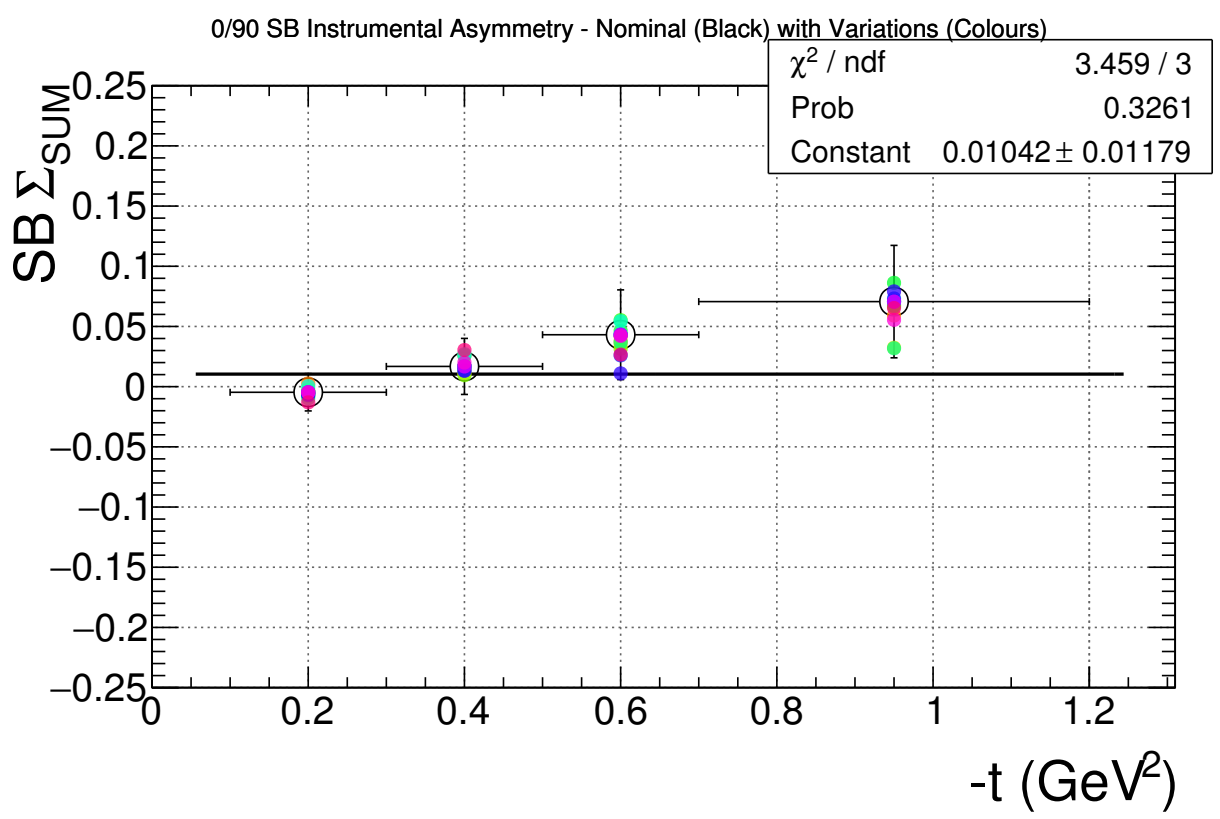

Figure B.11: 0/90 $\eta^{\prime}$ side-band region asymmetries for nominal (black open circles with error bars) and varied (coloured filled circles) cuts. Colours are as in the legend shown in Figure 5.12 (colour online). 


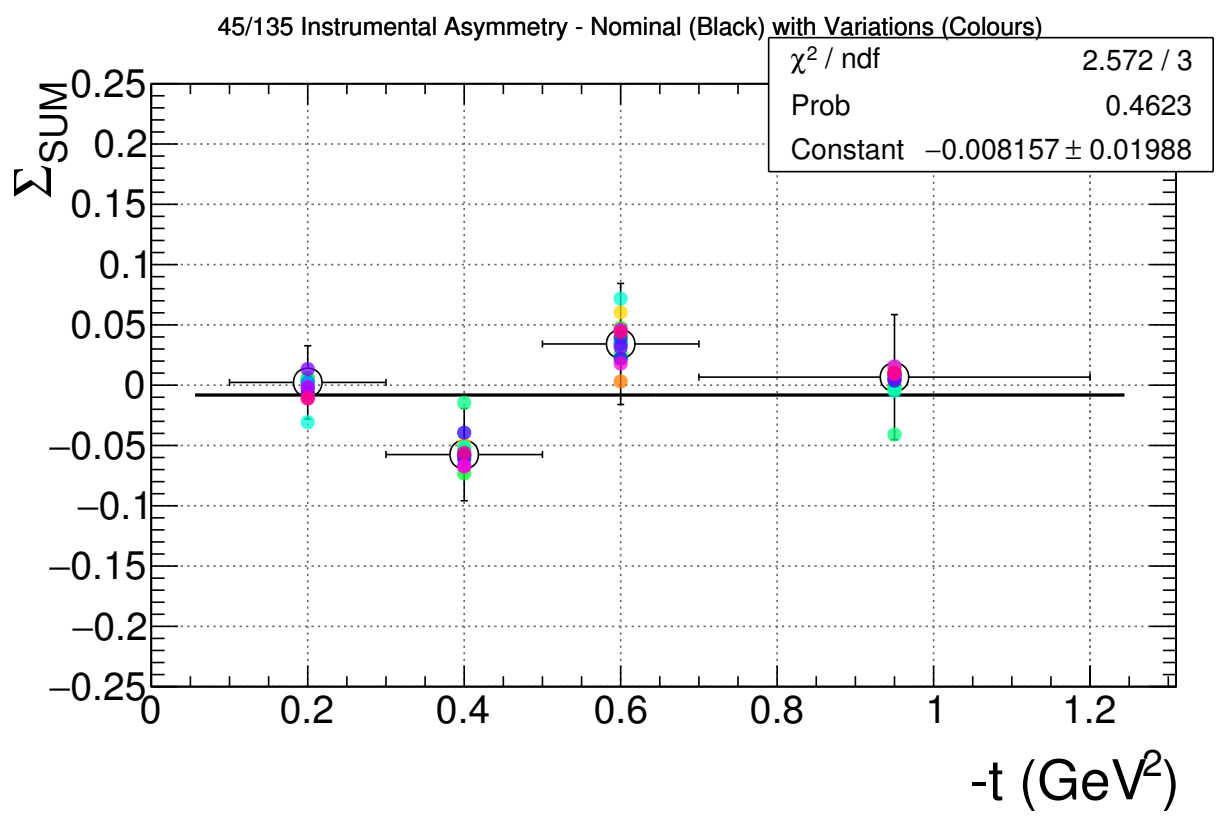

Figure B.12: $45 / 135 \eta^{\prime}$ peak region asymmetries for nominal (black open circles with error bars) and varied (coloured filled circles) cuts. Colours are as in the legend shown in Figure 5.12 (colour online).

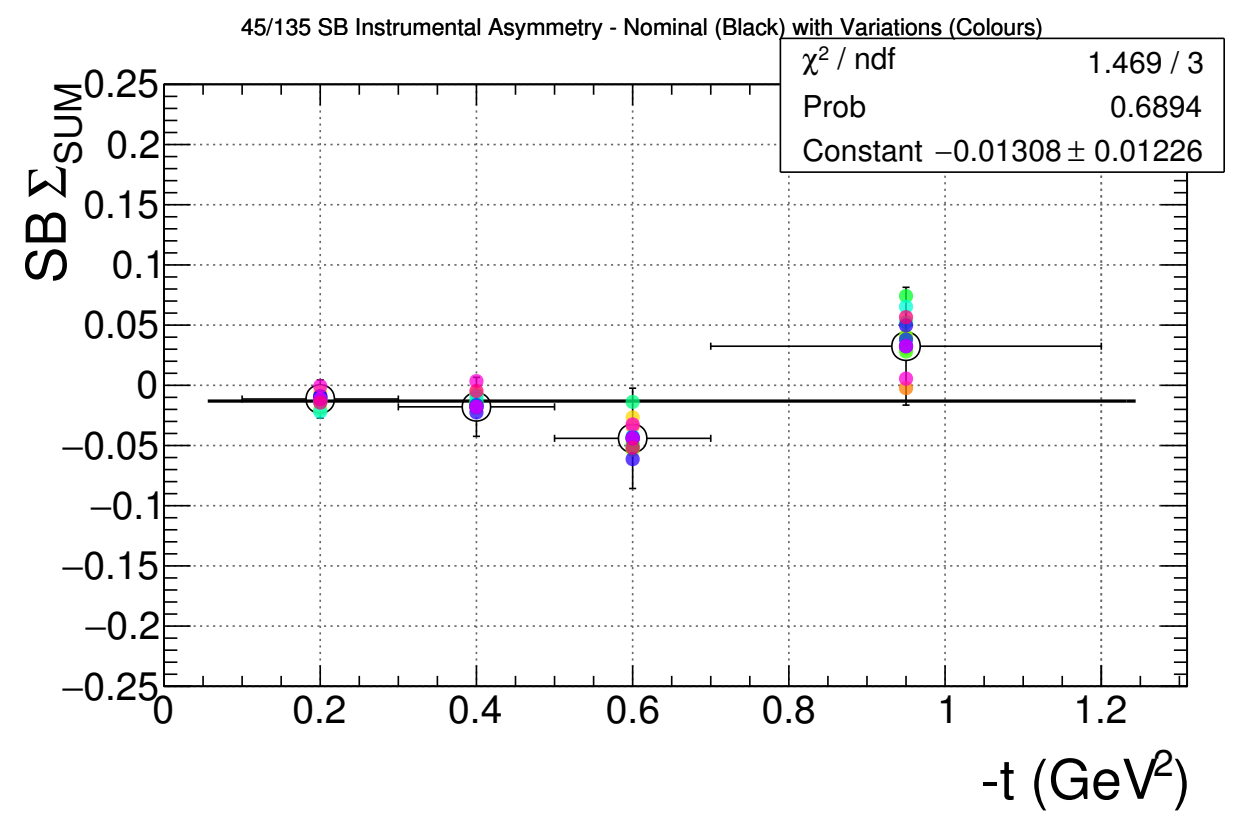

Figure B.13: 45/135 $\eta^{\prime}$ side-band region asymmetries for nominal (black open circles with error bars) and varied (coloured filled circles) cuts. Colours are as in the legend shown in Figure 5.12 (colour online). 


\section{Appendix $\mathrm{C}$}

\section{Summary of Beam Asymmetry Results}

\begin{tabular}{|c||c|c||c|c||c|c||c|c|}
\cline { 2 - 8 } \multicolumn{1}{c|}{} & \multicolumn{2}{c||}{$\eta \rightarrow 3$ piq } & \multicolumn{2}{c||}{$\eta \rightarrow 3$ pi0 } & \multicolumn{2}{c||}{$\eta \rightarrow 2 \mathrm{~g}$} & \multicolumn{2}{c|}{ Combined $\eta$} \\
\hline$t$ range $\left(\mathrm{GeV}^{2}\right)$ & $\Sigma_{\eta}$ & $\delta_{\Sigma_{\eta}}$ & $\Sigma_{\eta}$ & $\delta_{\Sigma_{\eta}}$ & $\Sigma_{\eta}$ & $\delta_{\Sigma_{\eta}}$ & $\Sigma_{\eta}$ & $\delta_{\Sigma_{\eta}}$ \\
\hline \hline $0.1<-t<0.3$ & 0.944 & 0.046 & 0.902 & 0.047 & 1.008 & 0.023 & 0.980 & 0.019 \\
\hline $0.3<-t<0.5$ & 0.977 & 0.048 & 1.019 & 0.047 & 0.990 & 0.027 & 0.994 & 0.021 \\
\hline $0.5<-t<0.7$ & 0.891 & 0.063 & 0.981 & 0.059 & 0.934 & 0.034 & 0.936 & 0.027 \\
\hline $0.7<-t<1.2$ & 0.929 & 0.063 & 0.879 & 0.064 & 0.854 & 0.031 & 0.870 & 0.026 \\
\hline
\end{tabular}

Table C.1: Asymmetries and statistical errors for the three $\eta$ decay channels separately and for the weighted average of the three $\eta$ results.

\begin{tabular}{|c||c|c|c|}
\hline$t$ Range $\left(\mathrm{GeV}^{2}\right)$ & $\Sigma_{\eta^{\prime}}$ & $\delta_{\Sigma_{\eta^{\prime}}}$ & $\delta_{\text {SYS }}$ \\
\hline \hline $0.1<-t<0.3$ & 0.873 & 0.066 & 0.030 \\
\hline $0.3<-t<0.5$ & 0.904 & 0.076 & 0.022 \\
\hline $0.5<-t<0.7$ & 0.882 & 0.097 & 0.036 \\
\hline $0.7<-t<1.2$ & 0.911 & 0.096 & 0.024 \\
\hline
\end{tabular}

Table C.2: Asymmetries, statistical errors, and systematic errors for the $\eta^{\prime}$ decay channel.

\begin{tabular}{|c||c|c||c|c||c|c||c|c|}
\cline { 2 - 9 } \multicolumn{1}{c|}{} & \multicolumn{2}{c||}{$\eta^{\prime} / \eta \rightarrow 3$ piq } & \multicolumn{2}{c||}{$\eta^{\prime} / \eta \rightarrow 3 \mathrm{pi0}$} & \multicolumn{2}{c||}{$\eta^{\prime} / \eta \rightarrow 2 \mathrm{~g}$} & \multicolumn{2}{c|}{$\eta^{\prime} /$ Combined $\eta$} \\
\hline$t$ range $\left(\mathrm{GeV}^{2}\right)$ & $\Sigma_{\eta^{\prime}} / \Sigma_{\eta}$ & $\delta_{\Sigma_{\eta^{\prime}} / \Sigma_{\eta}}$ & $\Sigma_{\eta^{\prime}} / \Sigma_{\eta}$ & $\delta_{\Sigma_{\eta^{\prime}} / \Sigma_{\eta}}$ & $\Sigma_{\eta^{\prime}} / \Sigma_{\eta}$ & $\delta_{\Sigma_{\eta^{\prime}} / \Sigma_{\eta}}$ & $\Sigma_{\eta^{\prime}} / \Sigma_{\eta}$ & $\delta_{\Sigma_{\eta^{\prime}} / \Sigma_{\eta}}$ \\
\hline \hline $0.1<-t<0.3$ & 0.925 & 0.083 & 0.967 & 0.085 & 0.866 & 0.073 & 0.891 & 0.070 \\
\hline $0.3<-t<0.5$ & 0.925 & 0.090 & 0.887 & 0.089 & 0.912 & 0.082 & 0.909 & 0.079 \\
\hline $0.5<-t<0.7$ & 0.990 & 0.130 & 0.900 & 0.124 & 0.945 & 0.115 & 0.943 & 0.107 \\
\hline $0.7<-t<1.2$ & 0.980 & 0.123 & 1.036 & 0.125 & 1.067 & 0.109 & 1.047 & 0.114 \\
\hline
\end{tabular}

Table C.3: Asymmetry ratios (of the $\eta^{\prime}$ results to the three $\eta$ results separately and to the weighted average of the three $\eta$ results) and associated statistical errors. 